\title{
RE-ROUTING PATHWAYS TO DEPRESSION: EFFECTS OF MINDFULNESS ON INTERNALIZED STIGMA, APPRAISALS, COPING AND DEPRESSIVE SYMPTOMS
}

\author{
by \\ Kelly-Lyn Christie \\ A thesis submitted to \\ the Faculty of Graduate and Postdoctoral Affairs \\ in partial fulfillment of the requirements for the degree of \\ Doctor of Philosophy \\ in \\ Psychology
}

Carleton University

Ottawa, Canada

C2013 Kelly-Lyn Christie 


\begin{abstract}
Despite increasing research and accessibility of treatment, depression remains a leading cause of global disease and disability, and depression rates among young adults continue to rise (WHO, 2007). Both trait mindfulness and mindfulness training have been inversely associated with depressive symptoms (e.g., Segal, Williams \& Teasdale, 2002), however, the processes underlying this effect are not fully understood. The goal of the present program of research was to identify the extent to which psychosocial variables (including internalized stigma, coping, appraisal and stressor responses) account for the relationship between mindfulness and well-being. In Study $1(N=368)$, trait mindfulness predicted lower endorsement of internalized stigma and greater endorsement of positive appraisals and adaptive coping repertoires among young adults, which in turn predicted a decrease in depressive symptoms. In Study 2, an analysis of both student $(N=372)$ and community $(N=275)$ samples revealed that trait mindfulness inversely predicted the use of emotion-focused coping techniques in response to a variety of interpersonal, financial, and workplace/academic stressors, while mindful coping was associated with greater perceptions of controllability and the use of problem-focused coping strategies. Study 3 sought to determine if trait mindfulness (Study $3.1 ; N=19$ ) and brief mindfulness training (Study 3.2; $N=87$ ) influenced psychological response (e.g., anxiety, affect), coping, and neuroendocrine response following an acute stressor task. Facets of mindful observing and awareness predicted lower baseline cortisol among participants, while nonreacting to experience predicted a diminished cortisol response from baseline to poststressor task. No differences in coping, psychological or neuroendocrine response were observed as a function of a brief mindfulness intervention, suggesting that longer
\end{abstract}


interventions may be needed to elicit beneficial effects. These results support an inverse relationship between trait mindfulness and negative appraisals, internalized stigma, and the use of emotion-focused coping strategies, and suggest that these variables might constitute important pathways in the relationship between trait mindfulness and depressive symptoms. Programs targeted at developing mindfulness skills might be particularly effective in mitigating physical and psychological responses to stressors among individuals at risk of depressive illness.

Keywords: Trait mindfulness, internalized stigma, coping, depressive symptoms, cortisol 


\section{Acknowledgments}

I would like to extend my sincerest thanks to my supervisor, Dr. Hymie Anisman. Your multiple contributions to my personal and professional development over the past eight years cannot be adequately described. Working with you has been a true privilege, and I am grateful for the time and resources you have invested in me and my work.

I would like to thank my committee members, Dr. John Telner, Dr. Florence Kellner, Dr. Kim Matheson, and Dr. Shawn Hayley for the time, support and valuable feedback they have contributed to this program of research. I am extremely grateful to Jerzy Kulczycki, Marzena Sieczkos, Thomas White and Carolyn Barkley for advice and expert assistance with lab equipment, blood collection procedures and processing assays, and to my colleagues and lab mates, who have offered invaluable support over the course of my doctoral research. I am especially indebted to Valerie Repta, Warren Caldwell, Jesse Howell, Deanna Whelan, Keltie MacDonald, Courtney Haskins, Opal McInnis, Robyn McQuaid, Rob Gabrys and Kate Raspopow for assistance running laboratory sessions and valuable input on methodology and analyses.

I would like to thank my family and friends for being a continual source of love and support. To Mom, Dad, Shannon, Sara and Jenn, to Georgia and Andre, and to the Kerr family, thank you for restoring my perspective when I needed it most. Finally, to my partner Ryan, thank you for believing that I was capable of seeing this journey to the end. Thank you for living and dancing with me, and for your unfailing positivity and encouragement each and every day. This accomplishment is yours as much as it is mine. 


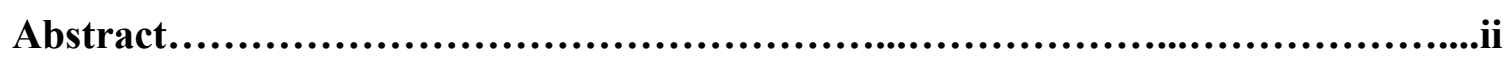

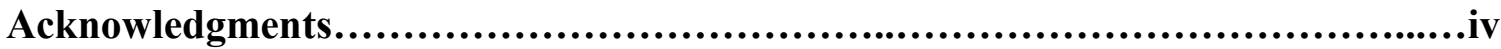

Table of Contents ...............................................................................................................

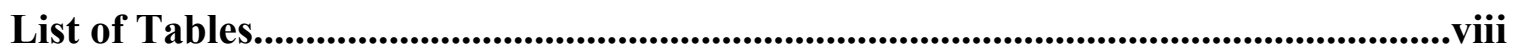

List of Figures.....................................................................................................................................

List of Appendices.............................................................................................................

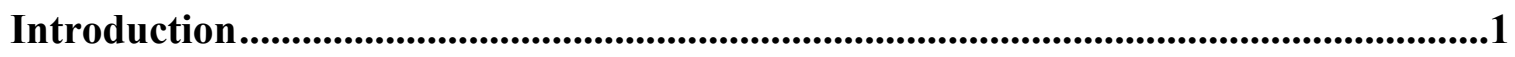

Negative Cognitions, Appraisals and Depressive Symptoms...................................5

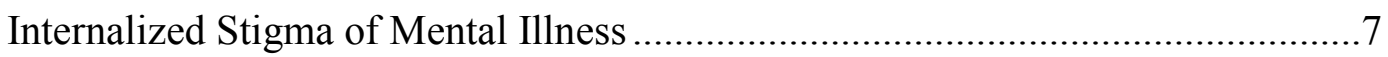

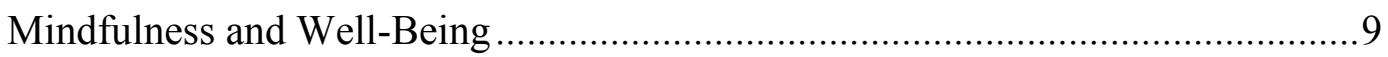

General Appraisal and Coping Mechanisms.....................................................14

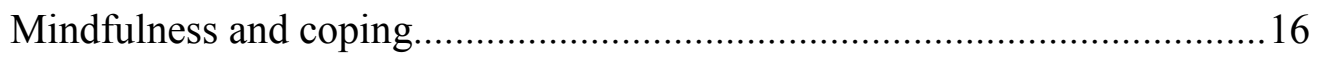

Physiological responses to acute stress.....................................................17

Immune and cytokine responses to acute stress ........................................18

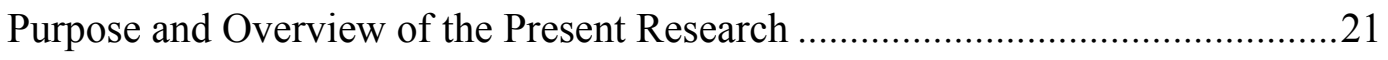

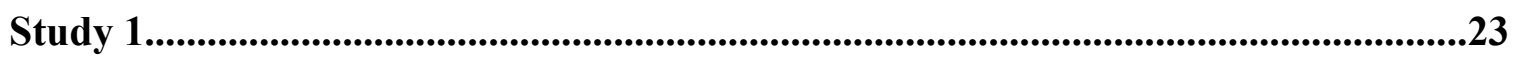

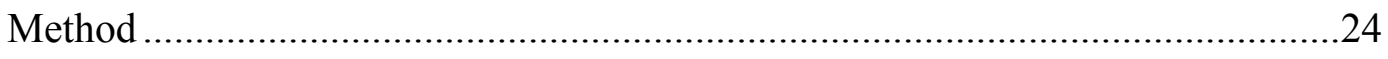


Results.

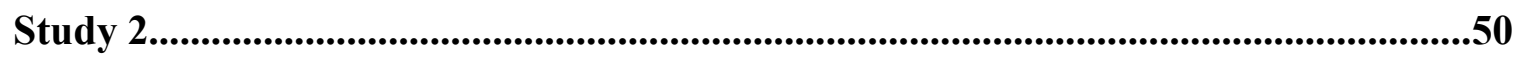

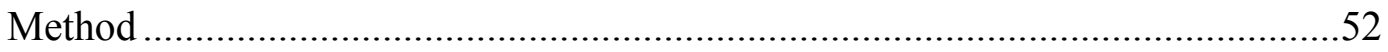

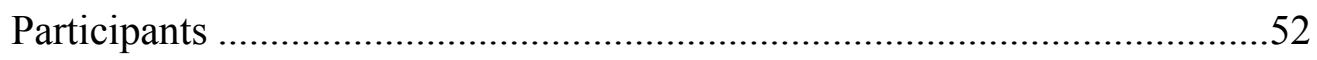

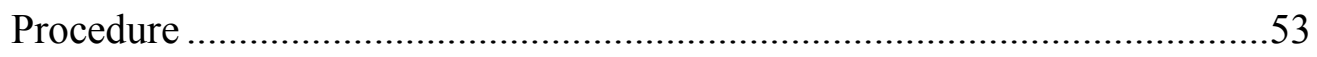

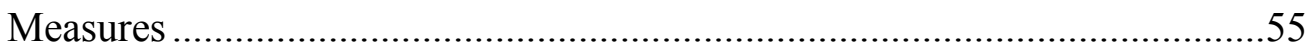

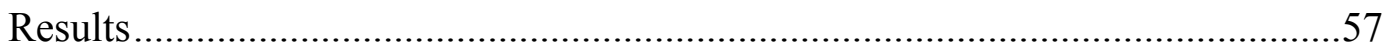

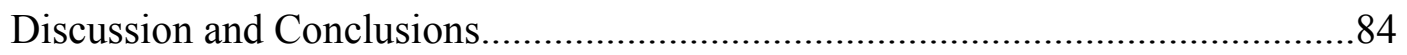

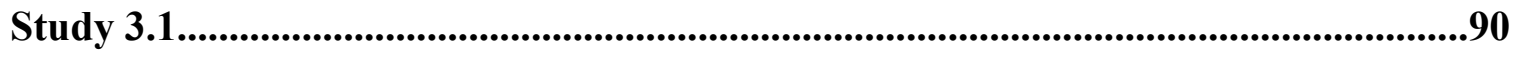

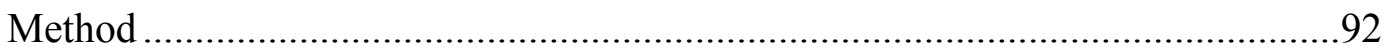

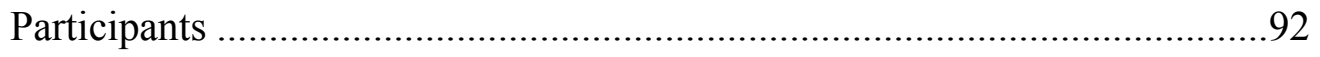

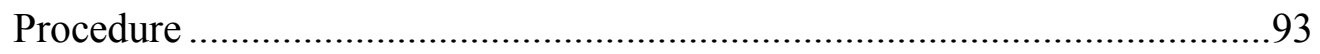

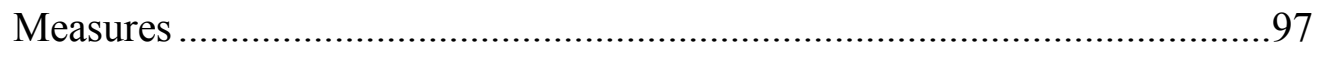


Results.

Discussion and Conclusions 108

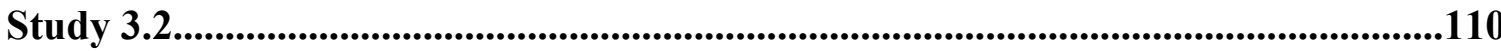

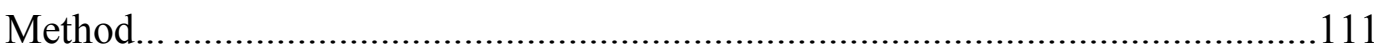

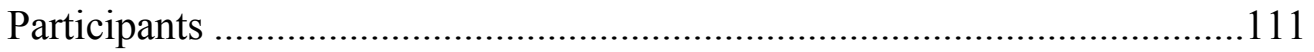

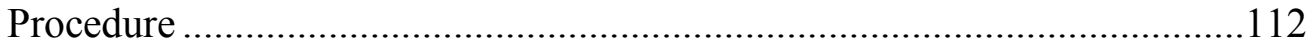

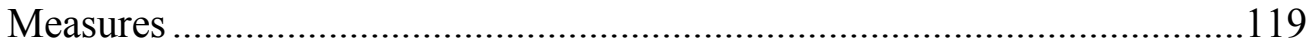

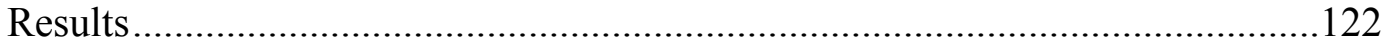

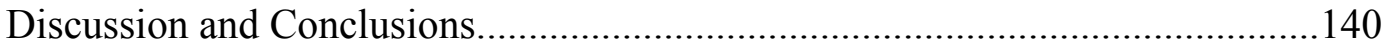

General Discussion................................................................144

Limitations.............................................................. 152

Implications of the present research....................................156

Conclusion............................................................ 158

References...............................................................................160

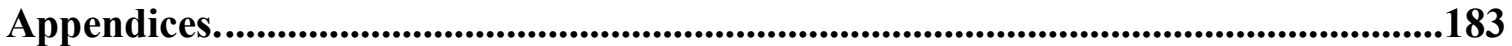




\section{List of Tables}

Table 1.1 Bivariate Correlations Between Trait Mindfulness (MAAS), Internalized Stigma (ISMIS), Depressive Symptoms (BDI), Appraisals, and Endorsement of Coping

Strategies Among Young Adults $(N=368)$. 32

Table 2.1 Summary of Internal Consistency Coefficients for Student $(N=368)$ and Community $(N=275)$ Samples. .57

Table 2.2 Summary of Bivariate Correlations, Means and Standard Deviations for SCOPE Subscales.

Table 2.3 Summary of Confirmatory Factor Analysis for SCOPE Subscales. .64

Table 2.4 Summary of Bivariate Correlations, Means and Standard Deviations for

Mindful Coping Items.

Table 2.5 Summary of Confirmatory Factor Analysis for Mindful Coping Items.....

Table 2.6 Summary of Comparative Fit Statistics for Three and Four-Factor SCOPE Models.

Table 2.7 Bivariate Correlations Between Trait Mindfulness (MAAS), Internalized Stigma (ISMIS), Depressive Symptoms (BDI), Appraisals, and Endorsement of Coping Dimensions Among Student $(N=368)$ and Community $(N=275)$ Samples. .73

Table 3.1.1 Summary of Internal Consistency Coefficients for Measures Assessing Coping, Internalized Stigma, Trait Mindfulness and Depressive Symptoms $(N=19)$ 100 
Table 3.1.2 Bivariate Correlations Between Unidimensional Trait Mindfulness (MAAS), Facets of Trait Mindfulness (FFMQ), Internalized Stigma, Depressive Symptoms, and

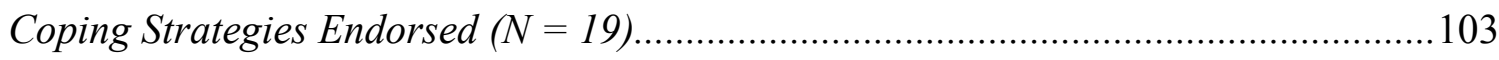

Table 3.2.1 Summary of Internal Consistency Coefficients for Measures Assessing Coping, Internalized Stigma, Trait Mindfulness and Depressive Symptoms

$(N=87)$

Table 3.2.2 Bivariate Correlations Between Trait Mindfulness, Internalized Stigma, Depressive Symptoms, Anxiety, Affect, and Coping Strategies Endorsed

$(N=87)$

Table 3.2.3 Summary of Descriptive Statistics for Coping Strategies Endorsed as a Function of Training Type (Mindfulness, Problem-Solving or Control) and Task Type

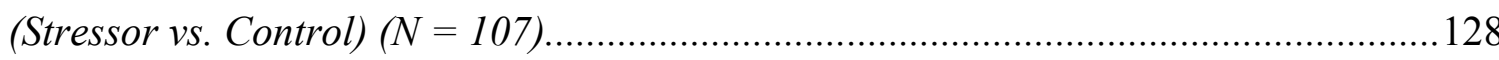




\section{List of Figures}

Figure 1.1. Simple slopes showing the moderating effect of trait mindfulness on the relationship between internalized stigma and depressive symptoms $(M \pm 1 S D)$. .35

Figure 1.2. Simple slopes showing the moderating effect of trait mindfulness on the relationship between emotion-focused coping and depressive symptoms

$(M \pm 1 S D)$ .36

Figure 1.3. Simple slopes showing the moderating effect of trait mindfulness on the relationship between distress appraisals and depressive symptoms $(M \pm 1 S D)$

Figure 1.4. Internalized Stigma (ISMIS) and coping styles (problem-focused, emotionfocused, and avoidant coping) as multiple mediators in the relationship between trait mindfulness and depressive symptoms.

Figure 1.5. Self-appraisals (mastery, self-esteem) as multiple mediators in the relationship between trait mindfulness and depressive symptoms

Figure 1.6. Situation appraisals (appraisals of threat, control, distress) as multiple mediators in the relationship between trait mindfulness and depressive

symptoms

Figure 2.1. Internalized Stigma (ISMIS) and coping styles (problem-focused, emotionfocused, avoidant and mindful coping) as multiple mediators in the relationship between trait mindfulness and depressive symptoms among students 
Figure 2.2. Internalized Stigma (ISMIS) and coping styles (problem-focused, emotionfocused, avoidant and mindful coping) as multiple mediators in the relationship between trait mindfulness and depressive symptoms in a community sample..............................83

Figure 3.2.1. Mean ( \pm SEM) State Anxiety, Positive Affect and Negative Affect Ratings as a Function of Task Type .......................................................................................130

Figure 3.2.2. Mean ( \pm SEM) Cortisol Response as a Function of Time of Assessment and

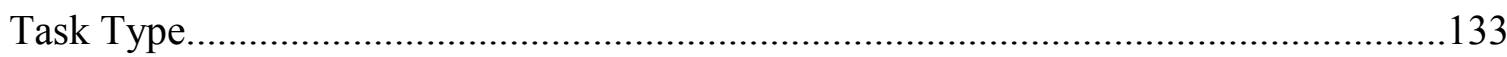




\section{List of Appendices}

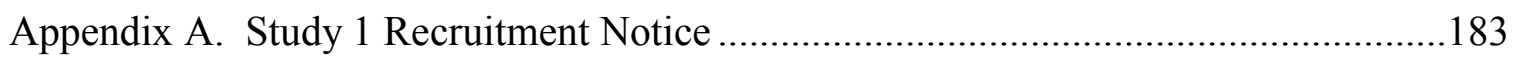

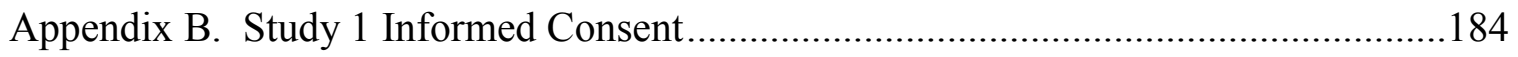

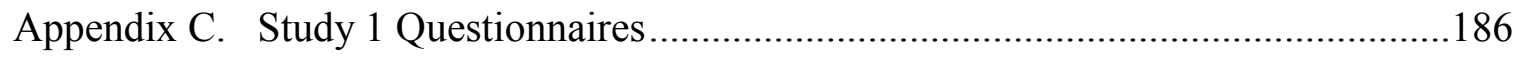

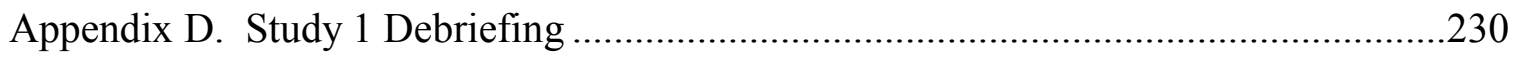

Appendix E. Study 2 Student and Community Recruitment Notices..................232

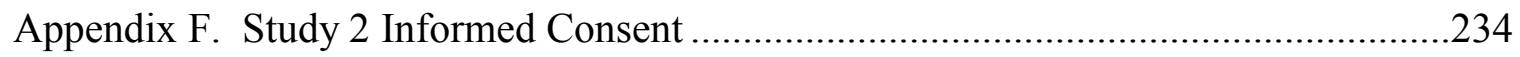

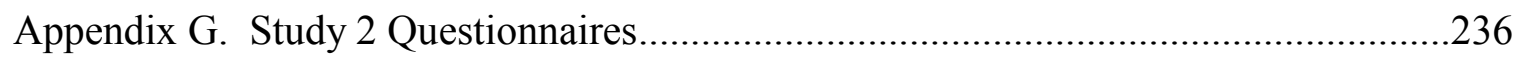

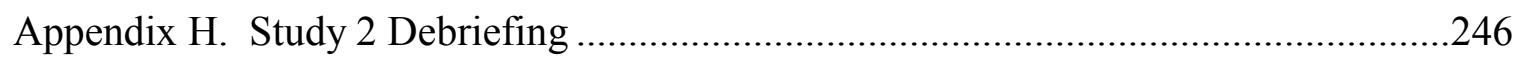

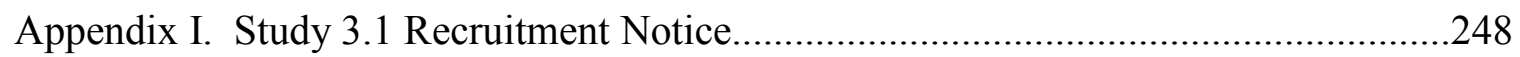

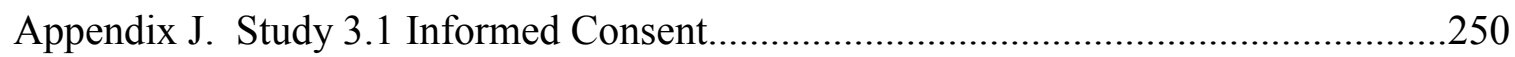

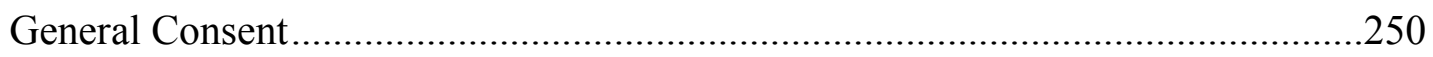

Consent for Blood Collection and Analysis..........................................................252

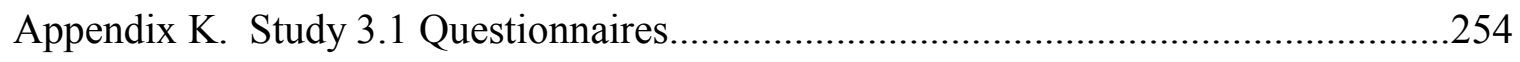

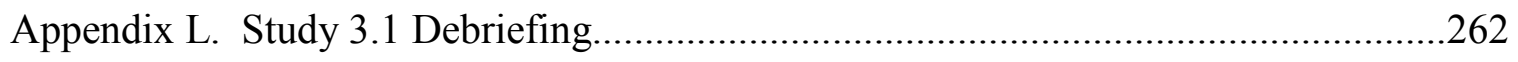

Appendix M. Study 3.2 Recruitment Email............................................................264

Appendix N. Study 3.2 Informed Consent (Session 1)..................................................26 
Appendix O. Study 3.2 Mindfulness Training Scripts........

Raisin Exercise

Mindful Meditation Exercise .269

3-Minute Breathing Space Exercise.

Appendix P. Study 3.2 Analytic Problem Solving Script and Materials

Problem-Solving Exercises.

Assessment of Problem-Solving Skills.

Appendix Q. Study 3.2 Control Condition Script and Materials.

Personal Activities Exercise .280

Activities and Hobbies Questionnaire. .282

Appendix R. Study 3.2 Mindfulness Handouts .286

Automatic Pilot. .286

The Breath. .287

Appendix S. Study 3.2 Homework Exercises. .288

Positive and Negative Events Calendar .288

Analytic Thoughts Calendar .289

Appendix T. Study 3.2 Debriefing (Session 1) .290 
Appendix U. Study 3.2 Informed Consent (Session 2)..........................................291

General Consent .......................................................................................291

Consent for Blood Collection and Analysis......................................................293

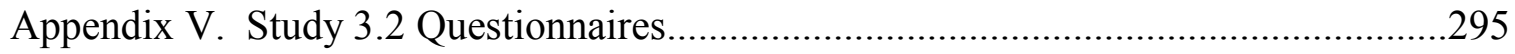

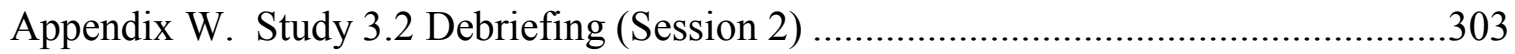




\section{Re-routing Pathways to Depression: Effects of Mindfulness on Internalized Stigma, Appraisals, Coping and Depressive Symptoms}

Depression is a debilitating illness, with an estimated lifetime prevalence of 12.2\% in Canada (Patten, Wang, Williams, Currie, Beck, Maxwell \& el-Guebaly, 2006; Ryan, 2005). The World Health Organization (WHO, 2007) has estimated that mood disorders, including Major Depression, are currently the fourth leading cause of diseaserelated disability globally, and are projected to constitute the second leading cause of disability by the year 2020. It appears that depressive symptoms are particularly acute among young adults between the ages of 15 and 25 years. Recent statistics indicate that Major Depressive Disorder is currently estimated to affect $0.4 \%$ to $8.3 \%$ of young adults in North America, with an additional $1.6 \%$ to $8.0 \%$ of this population meeting criteria for Dysthymic Disorder (Rood, Roelofs, Bögels, Nolen-Hoeksema \& Schouten, 2009).

Depressive symptoms have been associated with several negative outcomes in this population, including self-harm and anxiety, as well as increased frequency and severity of depressive episodes (see Rihmer, 2007; Ryan, 2005; Wang, Williams, Lavoroto, Schmitz, Dewa \& Patten, 2009; Weissman, Wolk, Wickramaratnre, Goldstein, Adams \& Greenwald, 1999). Suicidal thinking is prevalent among individuals with clinical depression, with the rate of suicidal ideation and attempted suicide reaching up to $56 \%$, and the rate of successful (i.e., completed) suicides estimated at $15 \%$ (Sher, Oquendo \& Mann, 2001). As such, understanding risk and resilience factors for depression is critical, particularly among young adults. 
In addition to physical effects of depression (e.g., lethargy, anxiety, changes in eating and sleeping patterns, etc.), depressed individuals often exhibit distorted cognitions regarding themselves and the world around them (e.g., Johnson, Johnson \& Petzel, 1992; Kovacs \& Beck, 1978; Panayiotou \& Papageorgious, 2007). In particular, the Cognitive Triad theory of depression (Beck, Rush, Shaw \& Emery, 1979) posits that depressed individuals tend to adopt pervasive, overarching and over-generalized negative views of the self and the world around them, and to hold negative expectations of the future. Considerable research has supported this model, indicating that negative self and world views are highly associated with depressive symptoms (e.g., Jones \& Day, 2008; Teasdale, 1983), and that low self-esteem and agency are strong predictors of depressive symptoms, particularly among young adults (e.g., Smith \& Betz, 2002).

One factor that may significantly aggravate these negative cognitions and contribute to the severity of depressive symptoms is stigma surrounding mental illness (e.g., Livingston \& Boyd, 2010; Moses, 2009). Although experiencing stigma may be damaging to individuals, it appears that internalized or self-stigma may predict additional impairments of self-esteem, as well as increased depressive symptoms (Holmes \& River, 1998; Lysaker, Roe \& Yanos, 2007; Moses, 2009; Rüsch, Corrigan, Todd \& Bodenhausen, 2010). In addition, there is evidence suggesting that, if left to germinate, these negative beliefs and attributions might become engrained within individuals, thus acting to perpetuate depressive symptoms (e.g., Teasdale, 1983). In view of the profound negative impact of self-stigma, it is important to identify variables that hinder the adoption of self-stigmas, and ultimately to develop strategies to protect individuals from internalizing negative beliefs. 
Based on the supposition that negative cognitions and appraisals are central to the development and maintenance of depressive symptoms, a great deal of research has focused on ways to deconstruct these cognitions and to build more adaptive thought and appraisal patterns (e.g., Lang, Moulds \& Holmes, 2009). These goals are central to Cognitive Behaviour Therapy (Beck, Rush, Shaw \& Emery, 1979), which focuses on teaching individuals to recognize negative thoughts and reactions, and to replace these with more adaptive responses. Less attention has been paid, however, to terminating these cognitions at their source and proactively identifying variables that inhibit individuals from internalizing negative self-information and scripts. One variable that is receiving increasing attention in this regard is mindfulness. Broadly defined, mindfulness refers to an individual's propensity to be attentive, aware and non-judgmental to events in the present moment (Brown \& Ryan, 2003). By remaining open, receptive and nonjudgmental in response to various situations, individuals may be less likely to ruminate or fixate on repetitive, non-productive thoughts and behaviours (Brown \& Ryan, 2003; Kabat-Zinn, 1990; Segal, Williams \& Teasdale, 2002). As such, mindfulness may represent an important approach for reducing negative appraisals, depressive symptoms, and self-stigmatization.

Although mindfulness has been associated with psychological well-being (e.g., Kabat-Zinn, 1990; 1994; Langer, 1989), limited information is available as to the mechanisms through which mindfulness influences well-being and depressive symptoms. Specifically, the extent to which mindfulness affects well-being via psychological processes (e.g., appraisals and the selection of coping strategies) and physiological processes (e.g., alterations of stress hormones, neurotransmitters or particular aspects of 
brain functioning following a stressor) is not known. In addition, although individuals may indicate high levels of trait mindfulness ${ }^{1}$ on self-report measures, this may not necessarily translate to the use of mindful actions and behaviours in daily life. When faced with stressors, for instance, these individuals might respond in emotional or nonmindful ways, and may show varying levels of mindfulness across different types of situations. Despite this possibility, limited research has examined the concordance between self-reported trait mindfulness and mindful behaviour (e.g., the use of mindful coping strategies), and it is not known whether individuals tend to employ mindful coping strategies differentially across various types of situations. If mindfulness is indeed endorsed as an effective coping strategy in different situations, this may enhance our understanding of mindfulness as a dynamic construct, and provide insight into the types of situations in which mindfulness supports well-being. Furthermore, if mindful coping does appear to promote adaptive responses in various contexts, it might be possible to develop this skill in order to circumvent negative appraisals, reduce internalized stigma, and improve well-being.

Based upon these outstanding questions, the goals of the present program of research were to:

a) examine the relationship between trait mindfulness, appraisals, physiological responses to stressors, the selection of coping strategies, and depressive symptoms, b) determine if trait mindfulness predicts reductions in internalized stigma,

\footnotetext{
${ }^{1}$ Trait mindfulness refers to a disposition characterized by receptive awareness and attention to present experience (Brown \& Ryan, 2003; Niemiec, Brown, Kashdan, Cozzolino, Breen, Levesque-Bristol \& Ryan, 2010).
} 
c) determine if trait mindfulness predicts mindful behaviours (i.e., the use of mindful coping strategies),

d) assess how the use of mindfulness as a coping strategy may vary across different situations and domains of functioning (e.g., academic/work, financial, personal), and

e) determine if mindfulness can be enhanced through brief training to reduce negative appraisals, internalized stigma, physiological responses to stressors, and depressive symptoms.

\section{Negative Cognitions, Appraisals and Depressive Symptoms}

According to the Cognitive Triad theory of depression (Beck, Rush, Shaw \& Emery, 1979), negative appraisals are fundamental to the onset and perpetuation of depressive symptoms. Depressed individuals tend to endorse these negative appraisals in a generalized manner, and across a broad range of life domains. Furthermore, it is posited that these cognitive patterns colour both inward and outward perception, and may hold in regards to both external factors (e.g., external events and situations, general world view, expectations for the future) and internal factors (e.g., appraisals of one's own worth, mastery, and coping ability) (Beck et al., 1979). Given the robust relationship between these negative appraisals and depressive symptoms, it is of interest to identify protective factors that guard against the adoption of such views.

Considerable research has supported a relationship between depressive symptoms and negative world-views, with world-views broadly defined as, "generalized beliefs about people, social groups, social institutions, the physical environment, or the spiritual world, as well as about categories of events and phenomena in the social world" (Leung 
\& Bond, 2008, p. 198). When negatively valenced, these views might include generalized feelings of injustice or cynicism about world events, negative assumptions regarding human nature, and feelings of suspicion, fear or distrust of others (Leung \& Bond, 2008). Importantly, it appears that the endorsement of these negative world-views may contribute to depressive symptoms and suicidal ideation (e.g., Chen, Wu \& Bond, 2009). Furthermore, if depressed individuals endorse these general appraisal patterns and habitually fixate on negative aspects of social situations, they might also develop negative expectations for events in their own lives. Specifically, if others are perceived to be untrustworthy and the unfolding of external events is perceived to be unjust, individuals might develop expectations that events in their own lives will turn out poorly, that they have little control over changing these outcomes, or that even benign situations pose a threat. As such, they might tend to appraise situations negatively in relation to non-depressed individuals, and these appraisals may influence subsequent behaviours, coping efforts and well-being.

In addition to interpreting external events negatively, individuals' self-appraisals and internalized narratives play a large part in determining how they view themselves, how they subsequently conduct themselves in general, and how they interact with others (e.g., McAdams, 1996). Indeed, it has been argued that "human beings are storytellers by nature, and the stories they tell about themselves and their social worlds form key aspects of their identities" (Adler, Wagner \& McAdams, 2007, p. 1180). Positive self-appraisals may include feelings of mastery (i.e., personal competence), autonomy, self-esteem and self-compassion (i.e., kindness and forgiveness of one's faults) (Neff, 2003). Previous research has shown that the endorsement of positive personal qualities (e.g., mastery) is 
highly predictive of well-being, while negative self-views are predictive of depressive symptomatology.

Individuals may lack confidence or self-esteem in different domains of functioning, or at different times in their lives. It is important to acknowledge that these feelings may be well-founded, and accurately reflect a need for learning (i.e., the individual may be aware that they lack the necessary skills, knowledge or experience to complete a given task), or may constitute an appropriate response to threat (i.e., they are not equipped to accept or complete a particular challenge). In this way, negative selfviews can be adaptive and serve a protective function in some situations, guarding individuals from acting out of turn or taking on challenges that they are not equipped to handle. For some people, however, these negative self-appraisals may dominate the narrative and eventually develop in to an automatic, stable appraisal pattern (NolenHoeksema, 1991). In fact, even if others point out strengths or positive qualities, these individuals may be unable to accept this information and integrate it in to their selfconcept (Stein \& Markus, 1996). This overarching negative self-concept, in turn, may predispose the individual to adopt poor coping strategies, and increase their vulnerability to depressive symptoms (e.g., Fennell, 1997). Even among non-depressed individuals, these cognitive patterns may serve as a vulnerability factor or marker for later depression.

\section{Internalized Stigma of Mental Illness}

Experiencing stigma from others (i.e., experiencing social rejection or discrimination on the basis of mental illness) constitutes a significant stressor, and stigmatized individuals may feel devalued and unable to cope with these events (Rüsch, 
Corrigan, Wassel, Michaels, Olschews, Wilkniss \& Batia, 2009). This public or enacted stigma has also been associated with perceptions of social isolation, lost opportunities (e.g., employment, housing, etc.), and depressive symptoms (Holmes \& River, 1998).

Although the influence of public stigma on depressive symptoms has received increasing attention, less research has focused on internalized stigma (also referred to as "felt" or "self-stigma"). Internalized stigma refers to the anticipation of stigma from others on the basis of one's personal characteristics (e.g., race, illness), the acceptance of social stereotypes and attitudes regarding one's illness, and integration of these stereotypes into one's self-concept (River \& Holmes, 1998; Rüsch, Corrigan, Todd \& Bodenhausen, 2010). Often, these internalized beliefs may be associated with feelings of shame regarding having a mental illness, or with a fear of experiencing public stigmas (Lundberg, Hansson, Wentz \& Bjorkman, 2009), which in turn may prompt individuals to isolate or withdraw themselves from social situations and interactions with others.

Research to date suggests that internalized stigma is particularly prevalent among individuals with mental illness, and may contribute to depressive symptoms as significantly as other public forms of stigma (e.g., social rejection, etc.). In one recent study, $45 \%$ of adults diagnosed with mental illness $(N=200)$ reported feeling as though others would devalue them for their illness, and reported isolating themselves from social interactions on account of this fear (Lundberg et al., 2009).

Internalized stigma has been associated with a host of negative physical and psychological health outcomes. Specifically, internalized stigma appears to predict a 
poor sense of self-worth and self-esteem, and may be associated with more negative general expectations for the future (Livingston \& Boyd, 2010; Moses, 2009). Indeed, both public and internalized stigma independently predict increases in depressive symptoms, and may compromise self-esteem and feelings of personal efficacy and empowerment (Lundberg et al., 2009; Rüsch, Corrigan, Todd \& Bodenhausen, 2010), as

well as eroding morale and potentially interfering with commitment to treatment (Ritsher \& Phelan, 2004). Importantly, once internalized, these beliefs appear resistant to change (e.g., Rüsch, Angermeyer \& Corrigan, 2005). As such, identifying factors that reduce or prevent the initial internalization of these beliefs is critical, and may promote downstream improvements in well-being, treatment efficacy and recovery from mental illness.

\section{Mindfulness and Well-Being}

The practice of mindful meditation has been hypothesized to enhance clarity of mind and self-awareness, qualities which may be intimately related to well-being and satisfaction with life (Kabat-Zinn, 1990; 1994). Interestingly, many depressed individuals report a feeling of isolation or detachment from others, and feeling as though they are living reactively rather than proactively, or as if they are acting on "autopilot" (e.g., Ben-Zeev \& Young, 2010). Church and Brooks (2009) coined the term subtle suicide to describe feelings of general apathy or withdrawal from life that may be observed among even non-depressed individuals, that predict low ratings on well-being and quality of life indices, and that, in extreme cases, may involve passive and apathetic self-destructive acts. This concept of detachment or numbing from daily life stands in sharp contrast to mindfulness, which is characterized by a sense of wakefulness and "clarity and vividness of current experience" (Brown \& Ryan, 2003, p. 823). 
Although the study of mindfulness is relatively new within the psychological literature, the concept of mindfulness is thought to have evolved from very old meditation-centered practices and traditions (see Brown \& Ryan, 2003; Kabat-Zinn, 1990). Within many Eastern traditions, and Buddhism in particular, active awareness and attention is encouraged in daily living, and meditation is often used to draw attention away from external thoughts and distractions (i.e., recent stresses, conflicts, etc.) and towards moment-to-moment internal processes and responses (i.e., breathing, muscular and autonomic activity). This practice is thought to "nurture greater awareness, clarity, and acceptance of present-moment reality....[and to] wake us up to the fact that our lives unfold only in moments" (Kabat-Zinn, 1990, p. 4).

Although mindfulness is generally defined in terms of attention and presentfocused awareness (Brown \& Ryan, 2003), these are only two hypothesized components of mindfulness. In addition, Kabat-Zinn (1990) proposed that mindfulness involves acceptance of one's reactions and responses, as well as non-judgment for these responses. Essentially, mindfulness itself refers to a way of experiencing events, rather than a specific method of cognitive processing. There is consensus in the early literature that mindfulness represents a distinct quality of consciousness, one that goes beyond surface cognition or awareness of situations and events. Instead, mindfulness involves living "receptively" in the present and being actively aware of one's physical and emotional responses to various situations, while suspending judgment of those responses (e.g., Brown \& Ryan, 2003; Deikman, 1982). Ideally, therefore, this practice allows an individual to reflect and observe unfolding situations at arms-length without becoming carried away or catastrophizing events, and to select a response rather than acting 
reflexively (Williams \& Swales, 2004). In this way, the practice of mindfulness focuses on changing the context of experience (i.e., one's perceptions and reactions to events) rather than the content of specific thoughts and emotions (Hayes \& Feldman, 2004; Palmer \& Rodger, 2009; Williams \& Swales, 2004).

Given the primary tenets of mindfulness and its focus on suspending judgment and emotional responding, the practice of mindfulness may help individuals to observe and process distressing thoughts and feelings without personal judgment, and to recognize and terminate negative appraisal loops and internal dialogues, allowing for more adaptive thoughts and behaviours (see Brown \& Ryan, 2003; Ryan \& Deci, 2000; Segal, Teasdale \& Williams, 2004; Segal, Williams \& Teasdale, 2002). As articulated by Jon Kabat-Zinn (1990); "By watching your thoughts without being drawn into them, you can learn something profoundly liberating...which may help you to be less of a prisoner of those thought patterns - often so strong in us - which are narrow, inaccurate, selfinvolved, [and] habitual to the point of being imprisoning...." (p. 94). Indeed, research has indicated that more mindful individuals tend to be more optimistic, to endorse more positive self-views (e.g., Aspinwall \& Richter, 1999), and to engage in more active coping strategies (i.e., problem solving) (Weinstein, Brown \& Ryan, 2009).

In contrast, non-mindfulness (or mindlessness) involves adopting automatic thoughts and responses, or responses that overlook relevant internal and external stimuli (i.e., thoughts, emotions, etc.) (Brown \& Ryan, 2003; Deci \& Ryan, 1980; Tart, 1994). Although some have argued that automatic behaviours are efficient and even preferable in some circumstances (i.e., to escape or avoid a dangerous situation, to conserve resources, etc.), automaticity might be less beneficial when employed as a general 
strategy. For instance, automatic responses might be appropriate for routine daily activities, or for tasks that do not require intensive mental processing, however, these same responses might lead an individual to overlook important features of their environment and interactions with others in different contexts. When individuals succumb to such automatic responses, they may be unaware that old habits (e.g., thoughts, emotions and bodily sensations) have become activated. Importantly, once these habitual responses are activated, it may then be too late for the individual to reassess the situation and respond adaptively (Palmer \& Rodger, 2009; Williams \& Swales, 2004).

In addition to promoting awareness and adaptive behavioural responses, the practice of mindfulness may actually re-wire neural circuits such that events are appraised less emotionally. Recent neuroimaging research has indicated that mindful awareness of stimuli can alter neural activity and influence information and emotion processing (e.g., Frewen et al., 2010; Teasdale, Segal, Williams, Ridgeway, Soulsby \& Lau, 2000). In a recent fMRI study (Herwig, Kaffenberger, Lutz \& Brühl, 2010) of healthy adults, participants were primed according to three conditions: cognitive selfreflection (i.e., thinking), emotion-introspection (i.e., mindful awareness), or an indifferent (i.e., neutral) condition. In the cognitive self-reflection trials, participants were asked to think about who they really are, and to reflect upon their goals and motivations. In the emotion-introspection condition, individuals were asked to reflect upon how they felt during the study, including being aware of any bodily sensations, emotions, or thoughts they experienced during the course of the research. In the indifferent condition, participants were given no specific instructions, and were asked 
only to focus on the presentation of neutral images. Individuals were then asked to respond to various images depicting positive, negative and neutral stimuli. When participants were explicitly asked to attend to their feelings and emotions (i.e., the emotion-introspection condition), they demonstrated less neural activity in the amygdala relative to either the cognitive or neutral conditions, indicating less emotional reactivity to stimuli (Herwig et al., 2010). Based on these results, the authors suggested that mindfulness, and in particular maintaining a present-focused awareness on bodily and emotional sensations, may facilitate emotion regulation and help to reduce the intensity of felt emotions.

There appear to be many benefits to practicing mindfulness, including gains in both physical (Kabat-Zinn, 1990; Langer, 1989; Zeidan, Johnson, Gordon \& Goolkasian, 2010) and psychological well-being (Brown \& Ryan, 2003; Herwig, Kaffenberger, Lutz $\&$ Brühl, 2010). In a recent longitudinal study, trait mindfulness was associated with relationship satisfaction and an increased capacity to cope with stressors in the context of romantic relationships (Barnes, Brown, Krusemark, Campbell \& Rogge, 2007). Similarly, reductions in reported stress, negative affect and anxiety were recently observed among women in late stages (i.e., third trimester) of pregnancy who participated in an 8-week mindfulness training session, relative to women in a "waitlist" control group (Vieten \& Astin, 2008). In other research, it was reported that participation in even a brief (i.e., 1 hour) mindfulness meditation session predicted reductions in negative mood and cardiovascular reactivity, compared to participation in a "sham" meditation session (Zeidan, Johnson, Gordon \& Goolkasian, 2010). Trait mindfulness has also been associated with lower depressive symptoms (Herwig, Kaffenberger, Lutz \& Brühl, 2010) 
and reductions in stress and somatic pain among individuals suffering from chronic illness, including cancer (Brown \& Ryan, 2003; Kieviet-Stijnen, Visser, Garssen \& Hudig, 2008). From a research perspective, these diverse benefits have prompted a great interest in identifying the mechanisms through which mindfulness influences well-being and positive health outcomes.

Since mindfulness involves a non-judgmental and receptive awareness of the present moment, the practice of mindfulness might help individuals to recognize and subsequently let go of negative automatic thoughts (e.g., Kabat-Zinn, 1990; Langer, 1989; Segal, Williams \& Teasdale, 2002; Teasdale, Segal \& Williams, 1995). In addition, present-moment awareness may allow individuals to view situations objectively (i.e., to remain focused on the issue at hand, rather than becoming emotional or anticipating future problems). As such, mindfulness might encourage individuals to appraise events more favourably, to endorse greater perceptions of control over external events, and consequently to adopt more active, problem-based coping strategies (Weinstein, Brown \& Ryan, 2009).

\section{General Appraisal and Coping Mechanisms}

Individuals may respond in very different ways when confronted with a stressor. Specifically, not all situations will be perceived as being equally stressful by all individuals, and the same stressor will not uniformly elicit the same coping response across all individuals (e.g., Carver \& Scheier, 1994). It has been suggested that individuals pass through two distinct appraisal stages when confronted with a potential stressor (Folkman, Lazarus, Dunkel-Schetter, DeLongis \& Gruen, 1986; Lazarus \& Folkman, 1984), which influence both how the event will be interpreted and how the 
individual will subsequently respond to the event. In the primary appraisal stage, a stressor is evaluated with respect to the level of threat or challenge it presents to the individual. If the event is perceived as a threat, a secondary appraisal is made, where the individual decides whether or not they have the coping resources necessary to handle or resolve the event. On the basis of these appraisals, particular coping strategies will be selected to deal with a stressor.

Broadly speaking, a typical coping response often includes both emotion-focused components (i.e., strategies aimed at managing or regulating emotions surrounding the event) as well as problem-focused components (i.e., strategies aimed at resolving or terminating the source of stress) (Lazarus \& Folkman, 1984). Either type of strategy may be beneficial, depending on the situation at hand. Specifically, problem-focused coping strategies might be particularly effective when dealing with stressors that are perceived as changeable or controllable (e.g., studying for an exam). In contrast, strategies aimed at mitigating emotional responses might be more appropriate in response to situations that are beyond one's subjective control, and in which problem solving is not feasible (e.g., the death of a loved one). Supporting this perspective, Folkman and Lazarus (1980) found that when coping resources were deemed to be unavailable at the secondary appraisal stage, there was a greater tendency for some individuals to employ emotionfocused coping strategies (e.g., rumination) in order to reduce or escape the emotional distress caused by the stressor. Conversely, if the individual believed they were able to mobilize appropriate coping strategies, there was a greater overall tendency towards the use of problem-focused coping strategies (e.g., problem solving, cognitive restructuring) in order to resolve or eliminate the source of stress. In this way, Lazarus and Folkman 
(1984) suggest that there is no single form of coping that is superior to others; instead, various strategies may be effective depending on the context and characteristics of the situation (and conversely, the same strategy may not be equally effective when applied broadly or rigidly to all types of stressors).

One particularly interesting coping mechanism that tends to co-occur with depression is rumination, defined as one's tendency of, "repetitively focusing on the fact that one is depressed, on one's symptoms of depression, and on the causes, meanings, and consequences of depressive symptoms" (Nolen-Hoeksema, 1991, p. 569). Essentially, rumination refers to a cyclic pattern of thinking which is often negatively valenced, and involves fixating on feelings of sadness, perceived mistakes that may have contributed to poor outcomes, or how events could have unfolded differently (i.e., in a more positive or favourable way). This pattern of thinking is non-productive and heavily focused on the past, and may occupy one's thoughts and overwhelm efforts to take action or think in more positive or constructive ways. Furthermore, it appears that a ruminative response style can become "automatic" (Nolen-Hoeksema, 1991), might contribute to increased feelings of sadness, regret or self-doubt, may hinder the use of active coping strategies, and may represent a risk factor for current and future depressive episodes among young adults with non-clinical depressive symptoms (Rood, Roelofs, Bögels, Nolen-Hoeksema $\&$ Schouten, 2009). In addition, the tendency to ruminate on negative emotions might reinforce negative appraisals that individuals hold regarding their own agency, worth and coping ability.

Mindfulness and coping. In addition to gains in well-being that may be afforded by actively and mindfully living in the present, the practice of focusing on the present 
moment may discourage emotion-focused coping tendencies, rumination and negative self-reflection. Since rumination represents a pattern of thinking that is heavily focused on the past and the experience of negative sensations (Nolen-Hoeksema, 1991), mindfulness (which fundamentally requires focusing and sustaining attention on the present moment) may be viewed as diametrically opposed to such thinking. In fact, mindfulness may encourage the use of more active coping strategies, as individuals are more likely to recognize and attend to issues as they arise, and not to push these issues aside or become distracted by other tasks. Furthermore, among individuals who do tend to endorse negative self-views, higher levels of trait mindfulness and the practice of mindful awareness might help to curb these appraisals, break negative self-appraisal and rumination loops, encourage adaptive coping responses, and encourage more objective reflection on one's thoughts and feelings (Teasdale, Segal, Williams, Ridgeway, Soulsby \& Lau, 2000). Supporting this contention, a recent study found that high-ruminating individuals demonstrated enhanced social problem solving skills when they were primed to view situations mindfully, rather than analytically (Sanders \& Lam, 2010). The authors suggested that by focusing on the present, individuals might be more likely to process information effectively, and less likely to feel anxiety (a future-oriented emotion) or ruminative regret (a past-oriented emotion) when faced with stressors or challenges (see also Heeren \& Philippot, 2011, for a discussion of mindfulness and rumination).

Physiological responses to acute stress. The appraisal of a stressor instigates a cascade of physiological responses that largely determine the extent and severity of one's subjective feeling of stress (e.g., Stroud, Salovey \& Epel, 2002). This process begins with activation of prefrontal cortical and amygdalar brain regions that are involved in 
stressor appraisal processes, and subsequent activation of the paraventricular nucleus (PVN) of the hypothalamus, which triggers the release of corticotrophin releasing hormone $(\mathrm{CRH})$ from the median eminence. The $\mathrm{CRH}$, in turn, stimulates the release of adrenocorticotropic hormone (ACTH) from the pituitary gland (see Antoni, 1986; Lamberts, Verleun, Oosterom, DeJong \& Hackeng, 1984). The presence of ACTH prompts the release of cortisol from the adrenal cortex, which facilitates active coping efforts. The circulating cortisol then serves a regulatory function, feeding back to the hypothalamus to inhibit the release of CRH, slow autonomic activity, and ultimately terminate the stress response (e.g., Sapolsky, Romero \& Munck, 2000). This process is adaptive in many ways, as it allows the mobilization of physical resources (i.e., increased blood flow to muscles, conversion of glucose to energy) that prepare the body for protective action. However, if activated chronically or for extended periods of time, excess cortisol may contribute to pathology and compromised HPA function (Sapolsky et al., 2000).

Immune and cytokine responses to acute stress. Several hormones and peptides have been implicated in the stress response. In recent years, research has increasingly focused on the role of inflammatory processes, as they might contribute to comorbidity of several stress-related illnesses including depression, anxiety, heart disease, diabetes, auto-immune disorders, and neurodegerative disorders such as Parkinson's and Alzheimer's disease (e.g., Anisman, Merali \& Hayley, 2008). Particular attention has been devoted to cytokines, which serve as signalling molecules within the immune system and help to regulate cellular processes, ranging from cell differentiation to the production of blood cells (e.g., Keicolt-Glaser \& Glaser, 2002). Several cytokines, 
including interleukin-1 (IL-1 $\beta$ ), interleukin-6 (IL-6), and tumor necrosis factor-alpha (TNF- $\alpha$ ) act specifically within the immune system, facilitating inflammatory processes that guard against injury and infection, as well as helping to destroy and eliminate damaged cells (e.g., Keicolt-Glaser \& Glaser, 2002). In contrast to these factors, interleukin-10 (IL-10) acts in an anti-inflammatory capacity, and thus serves to regulate (i.e., balance) the contribution of pro-inflammatory cytokines. This process is crucial to maintaining normal cellular functioning and immune responses (Keicolt-Glaser \& Glaser, 2002).

It appears that cytokine variations co-occur with changes in both stress hormones (i.e., cortisol) and depressive symptoms. Previous research has shown that stressors including early childhood trauma (Nunes, Watanabe, Morimoto, Moriya \& Reiche, 2010) may be associated with chronic dysregulation of the HPA axis (i.e., elevated or suppressed cortisol activity), as well as with elevations in pro-inflammatory cytokines including IL-1 $\beta$, IL-6, and TNF- $\alpha$. In addition, robust cytokine elevations have been observed in both human and animal models of depression (Raison, Capuron \& Miller, 2006), suggesting that bi-directional associations might exist between depressive symptomology, HPA and cytokine dysregulation, and that elevations in pro-inflammatory cytokines may serve as a biological marker for vulnerability to depression (Mossner, Mikova, Koutsilieri, Saoud, Ehlis, Müller, et al., 2007). Supporting the presence of these relationships, one recent study found both HPA and cytokine dysregulation in a sample of clinically depressed adults (Anisman, Ravindran, Griffiths \& Merali, 1999). Specifically, elevated levels of plasma ACTH and diminished cortisol responses were observed in 
individuals with atypical depression, and increases in IL-1 $\beta$ production were noted among dysthymic patients.

As in previous research, elevations in pro-inflammatory cytokines were correlated with depressive symptoms and duration of illness (Anisman, Ravindran, Griffiths \& Merali, 1999). Although such findings are correlational and do not suggest a causal relationship between cytokines and depression, studies that involve immunotherapy have indicated that certain cytokines may be causally related to depressive illness. Specifically, interferon- $\alpha$ (IFN- $\alpha)$, a cytokine used in the treatment of hepatitis C and some forms of cancer, has been shown to elicit major depressive illness in about $60 \%$ of patients (e.g., Almeida, Guindalini, Batista-Neves, de Oliveira, Miranda-Scippa, \& Quarantini, 2010; Lotrich, Rabinovitz, Gironda \& Pollock, 2007). The depression elicited by IFN- $\alpha$ has been related to serotonergic dysfunction, and is attenuated by antidepressant medications (reviewed in Anisman et al., 2008; Capuron et al., 2002; Raison et al., 2006). Furthermore, it seems that circulating cytokines are extremely sensitive to stressors (Danielson, Matheson \& Anisman, 2011), and that the effects of social stressors on biological processes and behaviour are particularly acute, contributing to the evolution of depression and anxiety (Anisman, 2009).

Although preliminary research has shown that mindfulness training might slow autonomic activity associated with stress (e.g., heart rate) (Zeidan, Johnson, Gordon \& Goolkasian, 2010), curb neurogenic inflammation (Rosenkranz, Davidson, MacCoon, Sheridan, Kalin \& Lutz, 2013) and stabilize the production of certain cytokines (IL-6, IL10) among women with early-stage breast cancer (Witek-Janusek, Albuquerque, Rambo Chroniak, Chroniak, Durazo-Arvizu \& Mathews, 2008), the extent to which mindfulness 
training benefits immune function and physiological circuits implicated in the processing of acutely stressful stimuli is not known. If mindfulness training is found to influence HPA and cytokine activity in response to acute stressors, in addition to calming autonomic reactivity, this might represent an effective strategy for reducing depressive symptoms and the onset of stress-related pathologies.

\section{Purpose and Overview of the Present Research}

It appears that negative self-appraisals (i.e., low self-esteem and mastery) and the internalization of stigmas are highly predictive of poor coping repertoires and depressive symptoms (e.g., Jones \& Day, 2008; Smith \& Betz, 2002). One variable that may help to weaken these relationships and encourage more adaptive self-appraisals and coping responses is mindfulness. If individuals are capable of remaining attentive and focused on the present, they might tend to appraise events in a more objective way, and to remain receptive to situations as they unfold. These individuals might also ruminate or focus on negative aspects of situations to a lesser extent than non-mindful individuals, as situations can be evaluated on their own merit without being compared to past or anticipated future events. Thus, one purpose of the proposed research was to assess the nature of the relationship between mindfulness and self-appraisals, and to examine how mindfulness influences the types of strategies individuals use for coping with stressful situations (Studies 1 and 2). Extending upon previously observed associations between mindfulness and well-being (e.g., Kabat-Zinn, 1990), we hypothesized that mindfulness would predict well-being indirectly via encouraging more positive appraisals (of the self and various situations), and the use of more active, problem-focused coping strategies in response to potentially stressful situations. 
A second aim of this research was to determine if trait mindfulness inhibits the internalization of stigma to confer resistance to depressive symptoms (Study 1 and 2). It was of further interest to identify specific aspects of mindfulness that might influence the adoption of stigma and depressive symptoms (Study 3.1 and 3.2). We hypothesized that trait mindfulness would predict reductions in internalized stigma, and that this reduction, in turn, would predict a lower frequency and severity of depressive symptoms. We further expected that specific facets of mindfulness, including non-judgment and acceptance, would discourage internalized stigma and among participants, thereby representing one pathway or mechanism through which mindfulness influences internalized stigma and the development of depressive symptoms.

Although trait mindfulness has been associated with well-being, it is possible that self-reported mindfulness may not translate into the practice of mindful behaviours (e.g., the use of mindful coping strategies) across different situations. To investigate this possibility, a second goal of Study 2 was to assess individuals' endorsement of mindfulness-based coping strategies across a variety of different types of stressful situations (e.g., personal, financial, academic/workplace stressors), and to examine the concordance between self-reported trait mindfulness and the endorsement of mindful coping behaviours.

If trait mindfulness is found to predict reductions in negative appraisals, internalized stigma and depressive symptoms (Study 1 and 2), and the use of mindfulness-based coping strategies predicts well-being across different situations (Study 2), it is of further interest to determine if mindfulness influences physiological responses (i.e., cortisol and cytokine activity) to acute stressors, and to determine if mindfulness can 
be enhanced among individuals to elicit these effects. As such, the final goal of this research was to determine if trait mindfulness promotes decreases in physiological responses following a stressor challenge (Study 3.1), and to determine if brief mindfulness training encourages more benign physiological responses to stressors, the use of mindful coping strategies, and resistance to internalized stigma and depression (Study 3.2).

\section{STUDY 1}

Mindfulness has been robustly associated with psychological well-being in various clinical and non-clinical populations (e.g., Kabat-Zinn, 1990; 1994; Langer, 1989), however, limited information is available regarding the mechanisms through which mindfulness influences well-being and depressive symptoms. In particular, the extent to which mindfulness affects well-being via psychological processes (e.g., appraisals and the selection of coping strategies) is not known. One possibility is that mindfulness, with its focus on present-moment awareness and acceptance, might encourage individuals to appraise situations more objectively, thereby helping to attenuate negative and/or emotional responses to stressors (Carmody, 2009; Teasdale et al., 2002). Mindful awareness may also allow individuals to distance themselves emotionally from stressors, and to select situation-appropriate coping responses, rather than responding to stressors rigidly or reflexively based upon emotional habit (e.g., Williams \& Swales, 2004).

Extending upon previous research (e.g., Lundberg et al., 2009; Rüsch, Corrigan, Todd \& Bodenhausen, 2010), Study 1 assessed whether negative self-appraisals and internalized stigma were associated with greater depressive symptoms and more negative 
appraisals of various situations, and whether trait mindfulness was associated with a weakening of these effects. We hypothesized that negative self-views, the use of avoidant or emotional coping styles, and the tendency to appraise potentially stressful situations in a negative manner (i.e., as personally threatening and uncontrollable) would be positively associated with depressive symptoms. We further expected that dispositional mindfulness would buffer these effects, such that the relationship between negative self-appraisals, internalized stigma, poor coping repertoires and the development of depressive symptoms would be moderated by levels of trait mindfulness. More specifically, we expected that the predictive relationship between these variables and depressive symptoms would be weakened among highly mindful individuals. To investigate potential indirect (i.e., mediated) pathways between mindfulness and depressive symptoms, we hypothesized that mindfulness would predict lower levels of internalized stigma and the use of more active coping strategies in response to stressors, which in turn would predict lower depressive symptoms.

\section{Method}

\section{Participants}

A total of 368 participants ( $n=102$ male, $n=266$ female) were recruited from the undergraduate Psychology student pool at Carleton University, and invited to participate in a study on individual difference variables (e.g., mastery, self-esteem), appraisals, coping strategies and well-being. Participants ranged in age from 17 to 49 years (M age $=20.79$ years, $S D=4.70$ years $)$. The majority of participants were Caucasian $(57.9 \%, n$ $=213)$, Middle Eastern $(10.9 \%, n=40)$ or East Asian $(9.5 \%, n=35)$, with remaining 
participants reporting African American/Canadian (5.4\%, $n=20)$, South Asian $(5.7 \%, n$ $=21)$, Hispanic/South American $(2.2 \%, n=8)$, Native American/Canadian $(1.6 \%, n=6)$ or "Other" ethnicity $(6.5 \%, n=24)$. Most participants were registered as full-time students $(90.8 \%, n=334)$, with the remainder of the sample registered as part-time $(3.8 \%, n=15)$ or special (i.e., non-degree) students $(5.4 \%, n=20)$. The majority of students in this sample $(67.4 \%, n=248)$ reported few to no symptoms of depression (BDI $\leq 9)$, while $22.3 \%(n=82)$ reported mild to moderate symptoms of depression $(\mathrm{BDI}=10-18), 8.7 \%(n=32)$ reported moderate to severe symptoms of depression $(\mathrm{BDI}=19-29)$, and $1.4 \%(n=5)$ reported severe depressive symptoms (BDI score $\geq$ $30)$.

\section{Procedure}

A recruitment notice and description of this study was posted on the online Psychology experiment system (SONA) at Carleton University (see Appendix A for online recruitment notice). Students signing up for this study were emailed and invited to participate in a session on campus at Carleton University. This study took approximately 90 minutes to complete. Upon arriving at the testing location, participants were reminded of the time required to complete the study and the requirements of the experimental session, and completed an informed consent form (see Appendix B). Potential participants were told that they would receive $1.5 \%$ toward their final grade in an introductory psychology class for participating in this study. Participants then completed a series of questionnaires (see Appendix C) assessing demographic information, selfesteem (RSES; Rosenberg, 1965), mastery (RWB-M; Ryff, 1989), depressive symptoms (BDI; Beck, 1967), internalized stigma regarding depressive symptoms (ISMIS; Ritsher, 
Otilingam \& Grajales, 2003), coping styles endorsed (SCOPE; Matheson \& Anisman, 2003), trait mindfulness (MAAS; Brown \& Ryan, 2003), and appraisals of ambiguous, potentially stressful situations (AASQ; Kelly, Matheson \& Anisman, 2003). Following completion of the questionnaire package, participants were provided with the debriefing sheet and a list of support services in the event they experienced any distress as a result of their participation in this study (see Appendix D).

\section{Measures}

Demographics. The demographic questionnaire was used to collect basic descriptive and classification information regarding participants, including age, sex, ethnicity, student status, marital status, and prior history of depressive symptoms.

Self-esteem. The 10-item Rosenberg Self-Esteem Scale (RSES; Rosenberg, 1965) was used to assess self-esteem among participants. Items (e.g., "I feel that I have a number of good qualities") were rated on a 7-point Likert scale, with -3 representing 'Strongly Untrue of Me' and +3 representing 'Strongly True of Me'. Responses were summed, with higher scores indicating higher levels of self-esteem. Internal consistency reliability was moderately high in the present sample, Cronbach's $\alpha=.78$.

Mastery. The 14-item Mastery subscale of the Ryff Well-Being Inventory (RWB-M; Ryff, 1989) was used to assess feelings of personal mastery endorsed by participants. Items (e.g., "I am quite good at managing the many responsibilities of my daily life") were rated on a 6-point Likert scale, with 1 representing 'Strongly Disagree' and 6 representing 'Strongly Agree'. Items 2, 3, 5, 8, 11, 12 and 13 were reverse scored. 
Responses were then summed, with higher scores indicating higher levels of perceived mastery. Internal consistency reliability for this scale was high, Cronbach's $\alpha=.88$.

Depressive symptoms. The 21-item Beck Depression Inventory (BDI; Beck, 1972) was used to assess the presence and severity of depressive symptoms among participants. This measure has been extensively validated with clinical and non-clinical samples (e.g., Beck, Steer \& Garbin, 1988; Steer, Ball, Ranieri \& Beck, 1999). Participants were asked to read 21 groups of statements describing varying degrees of depressive symptomatology. Each statement corresponds to a value, ranging from 0 (no feelings of depression) to 3 (extreme feelings of depression). For each group, participants were asked to select the statement that most closely reflects how they feel. Responses were summed, with higher scores representing greater depressive symptomatology. As in previous studies, internal consistency was high in the present sample (Cronbach's $\alpha=$ $.90)$.

Internalized stigma. The 14-item Internalized Stigma of Mental Illness Scale (ISMIS; Ritsher, Otilingam \& Grajales, 2003) was used to assess the extent to which participants self-stigmatize for depressive symptoms. In order to better reflect the population of interest, the original wording of this scale was modified for this study, such that original references to "severe mental illness" were replaced with more appropriate wording (e.g., "feelings of depression"). This scale is comprised of four subscales, assessing alienation (e.g., "People could not possibly understand how I feel when I'm depressed”), isolation (e.g., "I stay away from social situations when I'm feeling down”), stigma endorsement (e.g., "When I feel down, I can't contribute anything to society") and perceived discrimination experiences (e.g., "People often patronize me for feeling 
down”). Items were rated on a 5 point Likert scale, ranging from 0 ('strongly disagree') to 4 ('completely agree'). Mean scores were calculated for each subscale, and these subscale means were then combined to yield a total mean score. Higher mean scores represent greater internalized stigma for depressive symptoms. Internal consistency reliability was adequate for ISMIS subscales of Social Withdrawal $(\alpha=.67)$, Alienation $(\alpha=.80)$ and Discrimination Experiences $(\alpha=.66)$.

Coping styles. The 27-item Survey of Coping Profiles Endorsed (SCOPE; Matheson \& Anisman, 2003) was used to assess the use of various coping strategies among participants. Participants were asked to reflect upon stressors they had encountered in recent weeks, and to indicate the extent to which they used various strategies to resolve these stressors. Thirteen coping dimensions were assessed, including problem solving, social support seeking, avoidance, rumination, emotional expression, emotional containment, active distraction, passive resignation, humour, self-blame, otherblame, cognitive restructuring and religion. Items were rated on a 5-point Likert scale, ranging from 0 ('Never') to 4 ('Almost Always'). Mean scores were calculated for each of the 13 subscales, with higher scores representing greater endorsement of the given coping strategy.

In order to confirm the factor structure of the SCOPE, a factor analysis (PCA) was performed using a varimax rotation. A preliminary analysis of factor loadings suggested that religion did not load on any dimensions. Accordingly, this item was excluded from further analyses. Three factors emerged that accounted for $51.52 \%$ of variance: problem-focused coping (comprising problem solving, cognitive restructuring, active distraction, humour and social support seeking), emotion-focused coping 
(comprising self-blame, other blame, rumination, wishful thinking, emotional containment and emotional expression), and avoidant coping (comprising cognitive distraction/avoidance and passive resignation). Internal consistency reliability was adequate to good for all factors (problem-focused $\alpha=.75$; emotion-focused $\alpha=.73$; avoidant $\alpha=.81$ ).

Trait mindfulness. The 15-item Mindful Attention Awareness Scale (MAAS; Brown \& Ryan, 2003) was used to assesses individual differences in mindfulness and the frequency of mindful states. Items (e.g., "I could be experiencing some emotion and not be conscious of it until some time later", "I find it difficult to stay focused on what's happening in the present") were rated on a 6-point Likert scale, with 1 representing 'almost always' and 6 representing 'almost never'. Responses were summed, with higher scores indicating higher levels of trait mindfulness. Internal consistency reliability was good in the present sample, Cronbach's $\alpha=.82$.

Situation appraisals. The 29-item Appraisal of Ambiguous Situations Questionnaire (AASQ; Kelly, Matheson \& Anisman, 2003) was used to assess individuals' appraisals of various situations. Participants were asked to read a series of potentially stressful hypothetical scenarios relating to personal (i.e., a conflict with a romantic partner) or global (i.e., a hijacking) events. Participants were then asked to rate their appraisal of each scenario along four dimensions: Threat (e.g., "How threatening would this situation be to you?"), Distress (e.g., "How distressing would this situation be for you?"), Perceived Control (e.g., "How much control do you think you would have over the outcome of this event?"), and Expected Outcome (e.g., "What would you be most likely to think will happen?"). The perceived threat and distress dimensions were 
rated on a 5-point Likert scale, ranging from 1 (not at all) to 5 (extremely), with higher scores representing greater perceptions of threat and distress, respectively. The perceived control dimension was similarly rated on a 5-point Likert scale, ranging from 1 (no control) to 5 (complete control), with higher scores representing greater perceptions of control. Finally, the expected outcome dimension was rated on a 5-point Likert scale, where 1 represents the expectation of a positive outcome (i.e., "My friendships will grow stronger") and 5 represents the expectation of a negative outcome (i.e., "My friendships will fade away"). Responses were summed and a mean score calculated to represent perceived threat, distress, control and outcome for each of the Personal and Traumatic events categories. Cronbach's reliability coefficients were moderate to high for all subscales in this sample (Threat-Personal $\alpha=.90$; Distress-Personal $\alpha=.90$; ControlPersonal $\alpha=.89$; Outcome-Personal $\alpha=.86$; Threat-Global $\alpha=.74$; Distress-Global $\alpha=$ .74; Control-Global $\alpha=.73$; Outcome-Global $\alpha=.67)$.

\section{Results}

\section{Preliminary Analyses}

Data screening. Preliminary analyses were conducted in order to confirm normality of the variables of interest. Descriptive statistics, histograms and boxplots were examined for all variables of interest to identify missing or out of range values, and to assess normality of the distribution of variables. Following recommendations by Tabachnick and Fidell (2007), standardized values were calculated for all variables. Seven cases with values falling outside \pm 3 SD were considered extreme scores and removed from the analyses as outliers. 
Gender differences. A one-way ANOVA was performed to identify any preexisting differences in trait mindfulness as a function of participant gender. Consistent with previous research (e.g., Brown, Kasser, Ryan, Linley, \& Orzech, 2009; MacKillop $\&$ Anderson, 2007), neither males $(M=43.89, S D=8.40)$ nor females $(M=42.01, S D=$ 9.78) differed significantly on trait mindfulness, $F(1,366)=2.90, p=.09, n s$.

Bivariate correlations. Prior to assessing the predictive effects of trait mindfulness on appraisals, coping, internalized stigma and depressive symptoms, bivariate correlations were examined to determine the strength and direction of the relationship between variables of interest. As expected, trait mindfulness was strongly, negatively associated with depressive symptoms, internalized stigma of depressive symptoms, and the endorsement of emotion-focused coping strategies. Trait mindfulness was also negatively correlated with appraisals of threat and distress in response to potentially stressful situations, and positively associated with mastery, appraisals of control, and the endorsement of problem-focused coping strategies. A summary of bivariate correlations is presented in Table 1.1 
Table 1.1

Bivariate Correlations Between Trait Mindfulness (MAAS), Internalized Stigma (ISMIS), Depressive Symptoms (BDI), Appraisals, and Endorsement of Coping Strategies Among Young Adults $(N=368)$

\begin{tabular}{|c|c|c|c|c|c|c|c|c|c|c|c|}
\hline & 1 & 2 & 3 & 4 & 5 & 6 & 7 & 8 & 9 & 10 & 11 \\
\hline 1.Trait Mindfulness & -- & & & & & & & & & & \\
\hline 2.Distress Appraisals & $-.18 * *$ & -- & & & & & & & & & \\
\hline 3.Threat Appraisals & $-.20 * *$ & $.79 * *$ & - & & & & & & & & \\
\hline 4.Control Appraisals & $.22 * *$ & $-.22 * *$ & $-.22 * *$ & -- & & & & & & & \\
\hline 5.Problem-focused Coping & $.12^{*}$ & -.01 & -.01 & .07 & -- & & & & & & \\
\hline 6.Emotion-focused Coping & $-.32 * *$ & $.28^{* *}$ & $.32 * *$ & -.10 & $.29 * *$ & -- & & & & & \\
\hline 7.Avoidant Coping & $.12^{*}$ & -.02 & .03 & .08 & $.35^{* *}$ & $.11^{*}$ & -- & & & & \\
\hline 8.Internalized Stigma & $-.41 * *$ & $.32 * *$ & $.34 * *$ & $-.27 * *$ & -.12 & $.43^{* *}$ & $-.11 *$ & -- & & & \\
\hline 9.Depressive Symptoms & $-.43 * *$ & $.26^{* *}$ & $.18 * *$ & $-.23 * *$ & $-.16 * *$ & $.46^{* *}$ & $-.22 * *$ & $.55 * *$ & -- & & \\
\hline 10.Self-Esteem & -.04 & .10 & $.10 *$ & $-.15 * *$ & $.14 * *$ & $.15^{* *}$ & .05 & .05 & .04 & -- & \\
\hline 11. Mastery & $.39 * *$ & $-.27 * *$ & $-.18 * *$ & $.23 * *$ & $.32 * *$ & $-.33 * *$ & $.21 * *$ & $-.48 * *$ & $-.65 * *$ & .03 & -- \\
\hline
\end{tabular}

$* p<.05, * * p<.01$ 


\section{Main Analyses}

\section{Self-appraisals, coping, internalized stigma and depressive symptoms.}

Building upon previous research, it was hypothesized that negative self-appraisals (e.g., low self-esteem and mastery), poor coping styles and internalized stigma would significantly predict depressive symptoms among participants, and that these relationships would be weaker among individuals high in trait mindfulness. A preliminary analysis of residual scatterplots revealed adequate normality and homoscedasticity of study variables. Absence of multicollinearity and singularity between independent variables was additionally supported by relatively small standard errors and tolerance values close to 1 (Tabachnick \& Fidell, 2007).

As expected, multiple regression indicated that these combined variables significantly predicted depressive symptoms, $F(6,361)=71.21, p<.01$. An examination of specific parameters indicated that mastery $(\mathrm{B}=-.29, t=-9.60)$ and avoidant coping styles $(\mathrm{B}=-.61, t=-3.21, p=.001)$ inversely predicted depressive symptoms, while internalized stigma $(\mathrm{B}=.63, t=4.94, p<.01)$ and the endorsement of emotion-focused coping styles $(\mathrm{B}=.36, t=5.45, p<.01)$ positively predicted depressive symptoms. Neither self-esteem $(p=.75)$ nor problem-focused coping $(p=.57)$ predicted depressive symptoms in the present sample.

A series of moderated regression analyses were then performed to determine if these relationships were moderated by trait mindfulness. Specifically, since mindfulness is proposed to limit negative emotional responding and self-judgment (Kabat-Zinn, 1990), it was expected that the relationships observed between internalized stigma, 
emotion-focused coping and depressive symptoms would be weakened among participants who were high in trait mindfulness. Mean-centered predictor and moderator variables were entered in the first step of the analyses, and the centered interaction term was entered in the second step. These analyses revealed that trait mindfulness significantly moderated both the relationship between internalized stigma and depressive symptoms, $F \Delta(1,364)=11.27, R^{2} \Delta=.020, p=<.01$, and the relationship between emotion-focused coping and depressive symptoms $F \Delta(1,364)=12.93, R^{2} \Delta=.024, p<$ .01 .

To probe the direction of these significant interaction effects, simple slopes analyses were performed using an online interaction calculator developed by Preacher, Curran and Bauer (2006). Simple slopes analysis revealed that the slope of the regression line was significantly different from zero at $1 \mathrm{SD}$ above the mean of trait mindfulness $(t=$ $4.55, p<.01)$, at the mean of trait mindfulness $(t=9.67, p<.01)$, and at $1 S D$ below the mean of mindfulness $(t=-2.11, p=.04)$. The direction of these effects indicate that the relationship between internalized stigma and depressive symptoms was more pronounced for individuals who were low (-1 SD) in trait mindfulness, and that this effect was diminished for individuals with moderate to high trait mindfulness. In other words, participants who endorsed self-stigmas reported higher instances of depressive symptoms, but this only occurred when levels of trait mindfulness were low. In effect, higher mindfulness scores appeared to buffer individuals with internalized stigma from developing depressive symptoms (see Figure 1.1). 


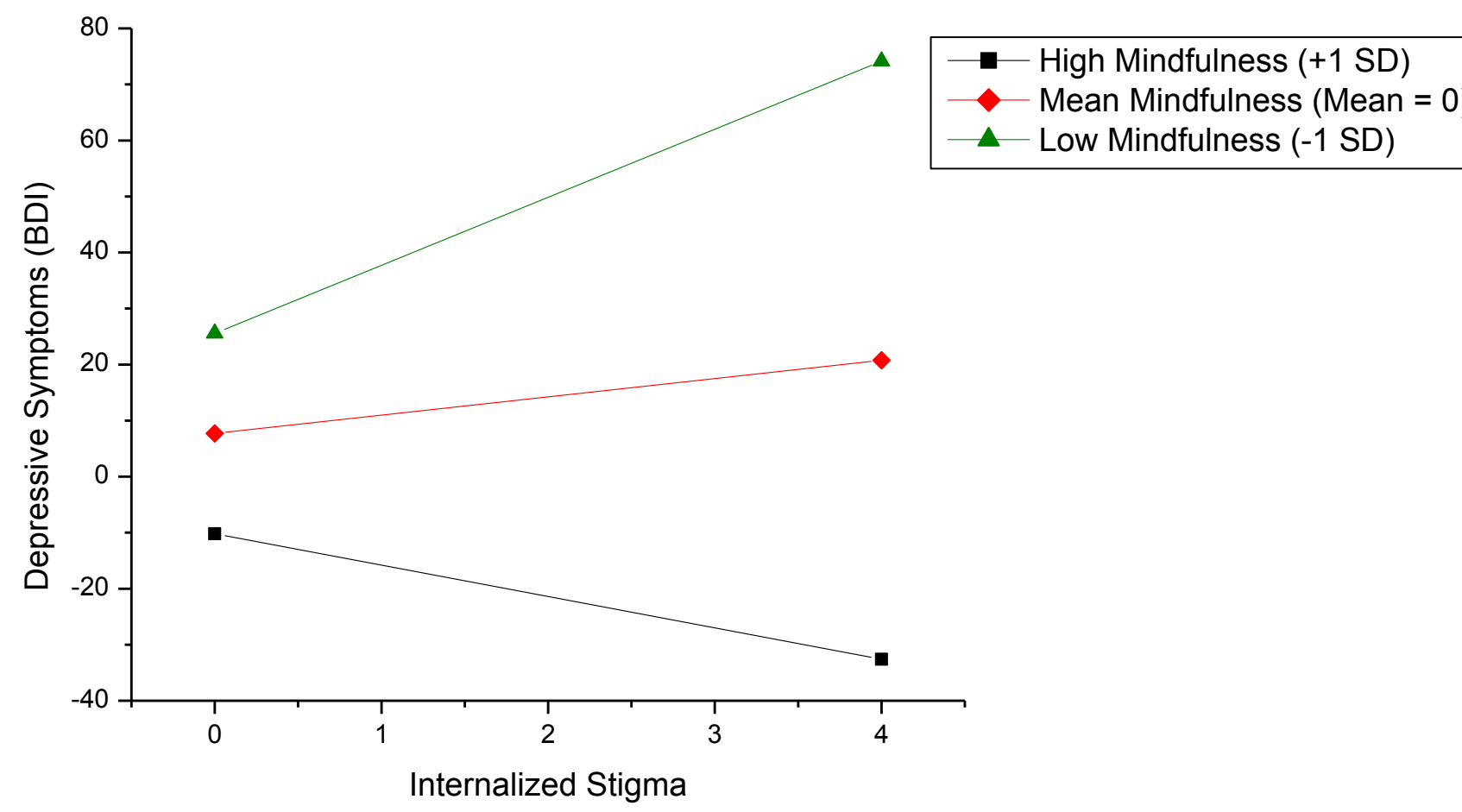

Figure 1.1. Simple slopes showing the moderating effect of trait mindfulness on the relationship between internalized stigma and depressive symptoms $(M \pm 1 S D)$.

Similarly, simple slopes probing the interaction between trait mindfulness and emotion-focused coping on depressive symptoms indicated that the slope of the regression line differed significantly from zero at $1 \mathrm{SD}$ above the mean of trait mindfulness $(t=-2.62, p=.01)$, at the mean of mindfulness $(t=8.26, p<.01)$, and at 1 $S D$ below the mean of trait mindfulness $(t=4.48, p<.01)$.

The direction of these effects revealed that the relationship between emotionfocused coping and depressive symptoms was weaker among highly mindful individuals. 
Specifically, emotion-focused coping strongly predicted depressive symptoms if individuals were low or moderate in their levels of trait mindfulness, but this effect was diminished among those high in trait mindfulness (see Figure 1.2).

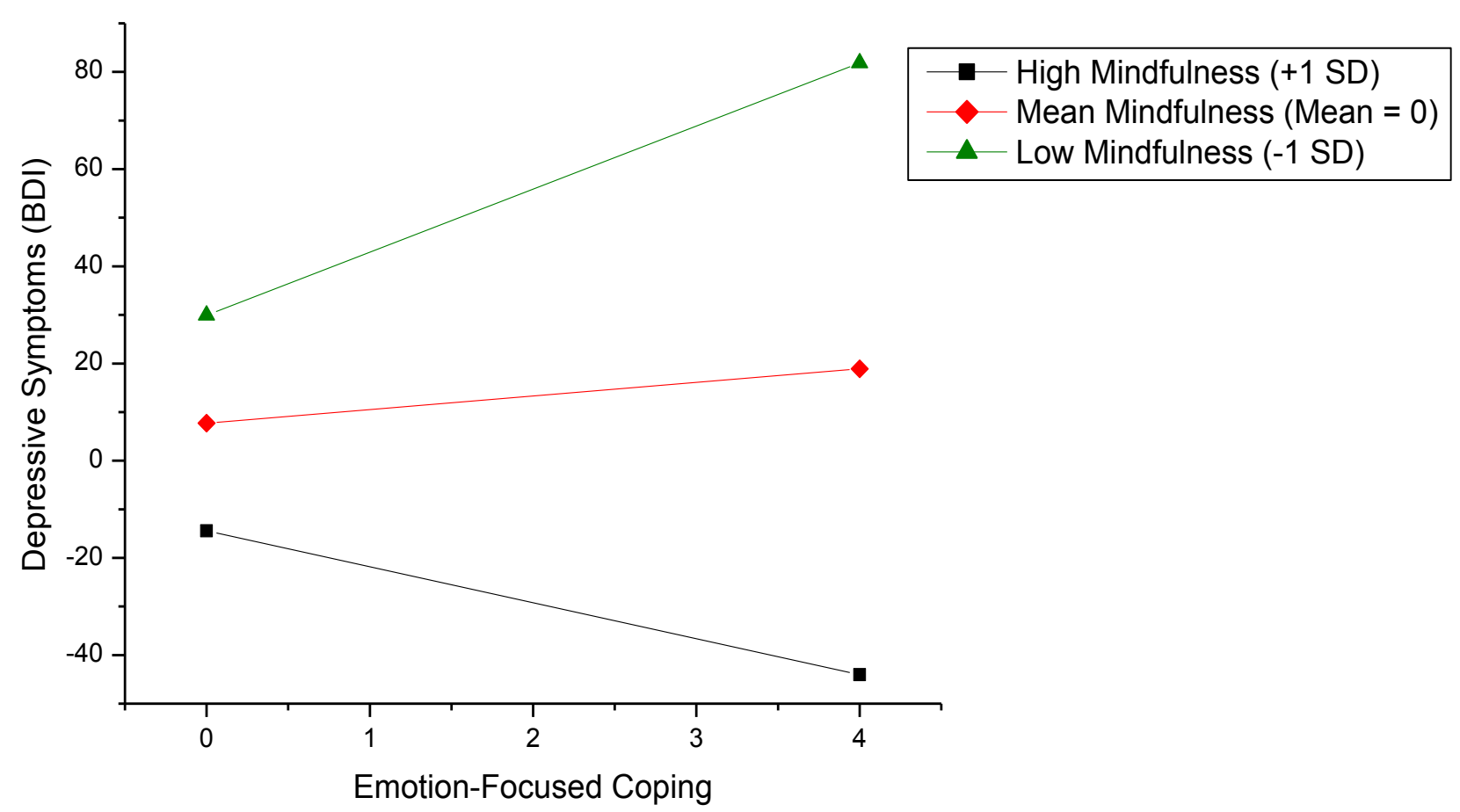

Figure 1.2. Simple slopes showing the moderating effect of trait mindfulness on the relationship between emotion-focused coping and depressive symptoms $(M \pm 1 S D)$.

Mindfulness and situation appraisals. Building on previous research (Beck, Rush, Shaw \& Emery, 1979; Jones \& Day, 2008; Teasdale, 1983), it was hypothesized that negative appraisals (e.g., appraisals of events as highly threatening, distressing and uncontrollable) would be associated with greater depressive symptomatology, and that 
this relationship would be weakened among highly mindful individuals. Again, a series of linear regression analyses were performed to assess this hypothesis.

As anticipated, appraisals significantly predicted depressive symptoms, $F(3,364)$ $=13.81, p<.01, R_{\text {adj }}^{2}=.10$. Specifically, distress appraisals positively predicted depressive symptoms $(\mathrm{B}=1.74, t=3.59, p<.01)$, whereas appraisals of control inversely predicted depressive symptoms $(\mathrm{B}=-1.03, t=-3.56, p<.01)$. Appraisals of threat were not significantly associated with depressive symptoms in the present sample $(\mathrm{B}=-.52, t=$ $-1.07, p=.29, n s)$

Again, moderated regression analyses were performed to determine if the positive relationship observed between distress appraisals and depressive symptoms varied as a function of trait mindfulness. Mean-centered appraisal variables were entered in the first step of the analysis, and the mean-centered interaction term was entered in the second step. These analyses indicated that trait mindfulness marginally moderated the relationship between distress appraisals and depressive symptoms $F \Delta(1,364)=3.52, R^{2} \Delta$ $=.007, p=.06$.

Simple slopes were analyzed to examine the direction of this interaction effect at $1 \mathrm{SD}$ above and below the mean of trait mindfulness, following recommendations by Aiken and West (1991), using interaction tools developed by Preacher, Curran and Bauer (2006). Supporting the marginally significant interaction term, simple slopes revealed that the slope of the regression line was not significantly different from zero at $1 S D$ above the mean of trait mindfulness $(t=-1.39, p=.16)$. The slope of the regression line was significantly different from zero, however, at mean levels of trait mindfulness $(t=$ 
$3.98, p<.01)$, and at $1 S D$ below the mean of trait mindfulness $(t=2.34, p=.02)$. This result suggests that although high levels of trait mindfulness may not influence the impact of distress appraisals on the development of depressive symptoms, the risk of developing depressive symptoms is amplified among individuals who both appraise situations as distressing and possess moderate to low levels of trait mindfulness (see Figure 1.3 for a summary of simple slopes analysis).

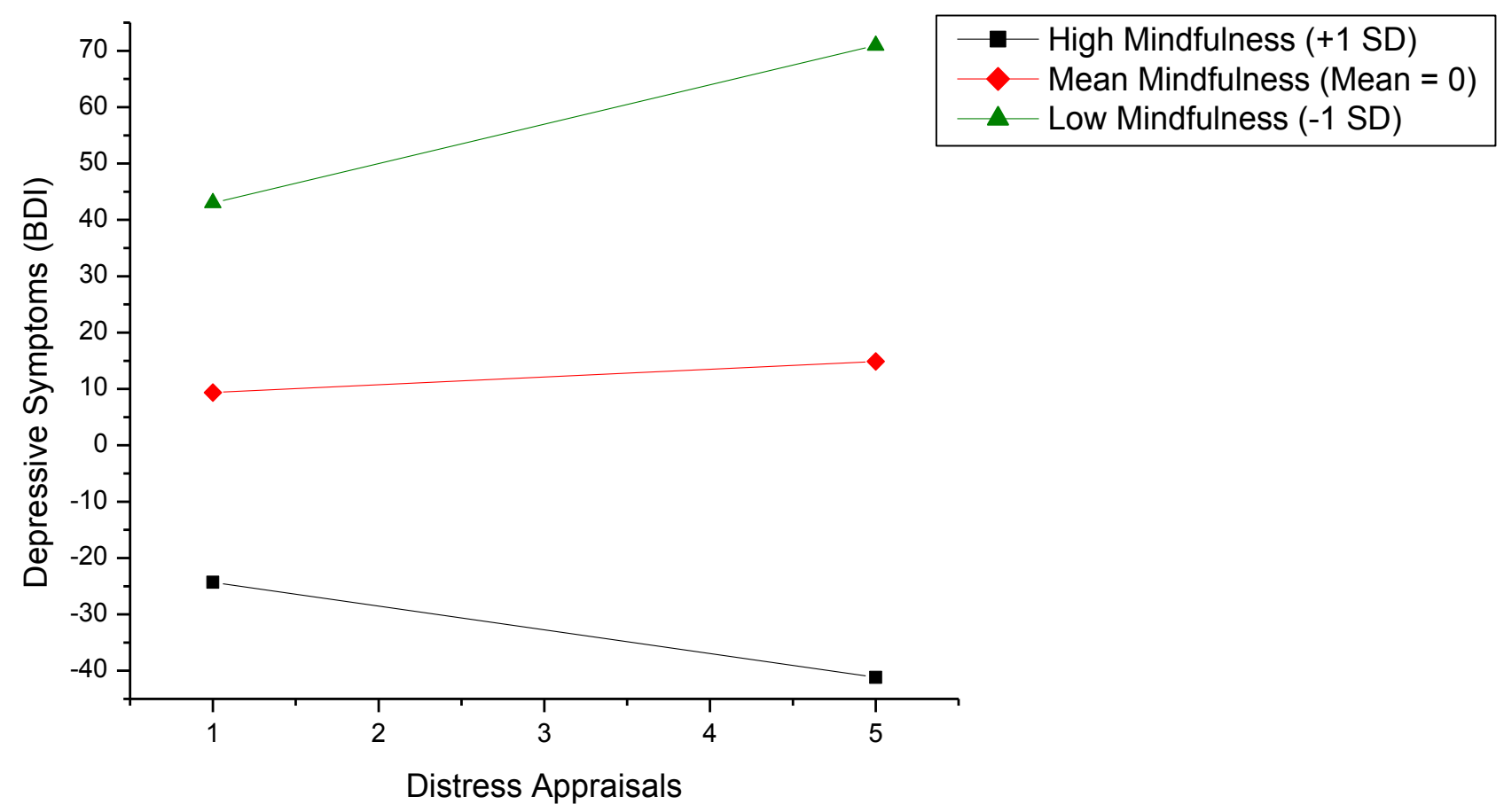

Figure 1.3. Simple slopes showing the moderating effect of trait mindfulness on the relationship between distress appraisals and depressive symptoms $(M \pm 1 S D)$. 
Mediating effects of internalized stigma and coping style. In addition to understanding how varying levels of trait mindfulness interact with appraisals, coping and internalized stigma to predict depressive symptoms, it is of interest to identify specific pathways through which mindfulness influences depressive symptoms among young adults. Based upon previous research (e.g., Weinstein et al., 2009), it was hypothesized that the negative relationships observed between mindfulness and depressive symptoms would be partially accounted for by coping style. Specifically, it was hypothesized that individuals higher in trait mindfulness would endorse greater use of problem-focused coping strategies, and diminished use of emotion-focused and avoidant coping strategies. Since mindfulness involves present-moment awareness and non-judgment (e.g., Kabat-Zinn, 1990), it was further hypothesized that mindful individuals would be less likely to internalize negative self-information, stereotypes and stigmatizing views towards depressive symptoms, and that this in turn would predict decreases in depressive symptoms. To test these hypotheses, a multiple mediation analysis was performed using Preacher and Hayes' (2008) macro for multiple mediation with 1000 iterations.

Trait mindfulness scores significantly and positively predicted participants' endorsement of problem-focused coping strategies $(\mathrm{B}=.03, t=2.35, p=.02)$ and avoidant coping strategies $(\mathrm{B}=.02, t=2.35, p=.02)$, and inversely predicted both internalized stigma $(\mathrm{B}=-.11, t=-8.55, p<.01)$ and the endorsement of emotion-focused coping strategies $(\mathrm{B}=-.17, t=-6.45, p<.01)$. As expected, all proposed mediating variables significantly predicted depressive symptoms, with problem-focused coping $(\mathrm{B}=$ $-.45, t=-3.33, p=.01)$ and avoidant coping $(\mathrm{B}=-.73, t=-3.54, p<.01)$ negatively 
predicting depressive symptoms, and emotion-focused coping $(\mathrm{B}=.50, t=7.02, p<$ $.01)$ and internalized stigma $(\mathrm{B}=.88, t=6.36, p<.01)$ predicting increased symptomatology. The direct effect of trait mindfulness on depressive symptoms was also significant, indicating that higher levels of trait mindfulness predicted lower levels of depressive symptoms among participants $(\mathrm{B}=-.13, t=-3.73, p<.001)$.

An examination of indirect effects revealed that the overall multiple mediation model was significant, $F(5,362)=58.35, p<.01)$. The model including all mediators accounted for $43.86 \%$ of the variance in depressive symptoms. An analysis of individual parameters indicated that internalized stigma significantly mediated the relationship between trait mindfulness and depressive symptoms. Specifically, higher trait mindfulness predicted reductions in internalized stigma, which in turn predicted a decrease in the prevalence and severity of depressive symptoms reported by participants $(C I=-.14,-.06)$. Similarly, problem-focused coping $(C I=-.04,-.002)$, emotion-focused coping $(\mathrm{CI}=-.13,-.05)$ and avoidant coping strategies $(\mathrm{CI}=-.04,-.003)$ all significantly mediated the relationship between trait mindfulness and depressive symptoms. As hypothesized, the direction of these effects indicated that higher levels of mindfulness predicted lower endorsement of emotion-focused coping strategies, and higher levels of problem-focused coping, each of which predicted decreases in depressive symptoms (see Figure 1.4 for a summary of multiple mediation model). 


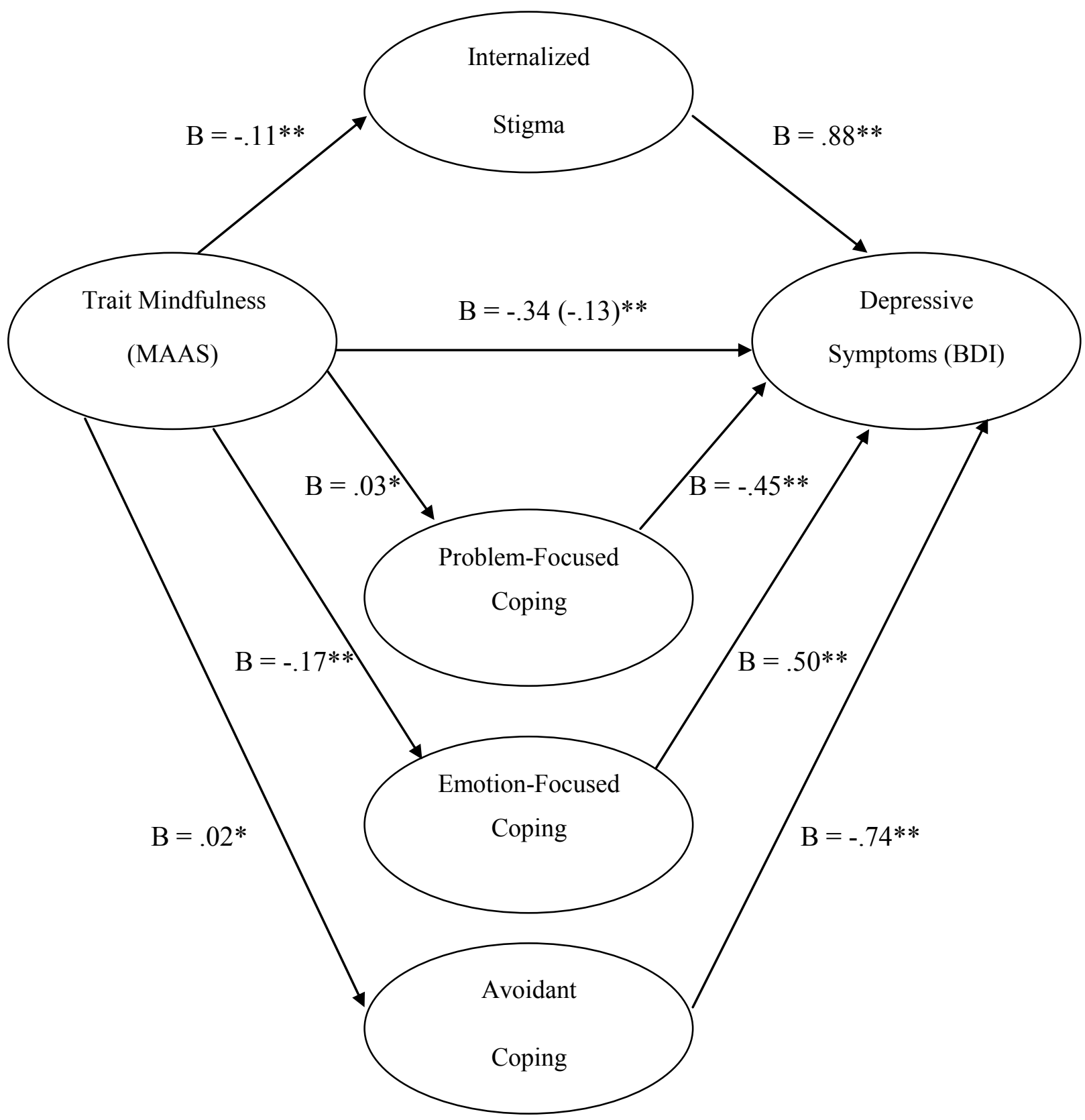

Figure 1.4. Internalized Stigma (ISMIS) and coping styles (problem-focused, emotionfocused, and avoidant coping) as multiple mediators in the relationship between trait mindfulness and depressive symptoms $\left({ }^{*} p<.05,{ }^{* *} p<.01\right)$. 
Mediating effects of appraisal. Considering previous research on mindfulness, coping and stress reactivity (Weinstein et al., 2009), it was hypothesized that individuals high in trait mindfulness would tend to perceive and evaluate potential stressors objectively, without becoming carried away or exaggerating the level of threat inherent in the situation. Specifically, it was hypothesized that mindful individuals would report greater perceived control over potentially stressful events, and lower levels of distress and threat in response to potential stressors. These positive appraisals, in turn, were expected to inversely predict depressive symptoms. Based on previous research (e.g., Smith \& Betz, 2002), we further expected that trait mindfulness would foster more positive self-appraisals (e.g., higher self-esteem and feelings of mastery) through encouraging greater self-acceptance and non-judgment. These positive self-appraisals were also expected to negatively predict depressive symptoms. To investigate these pathways, two separate multiple mediation models were examined using bootstrapping techniques outlined by Preacher and Hayes (2008), with self-appraisals and situation appraisals serving as mediators in the relationship between trait mindfulness and depressive symptoms, respectively.

Self-appraisals. The first multiple mediation model revealed that trait mindfulness scores positively predicted mastery among participants $(\mathrm{B}=.46, t=8.18, p$ $<.01)$, but did not significantly predict self-esteem $(\mathrm{B}=-.02, t=-.84, p=.40)$. Mastery also significantly predicted depressive symptoms, $(\mathrm{B}=-.38, t=-13.57, p<.01)$. As in the previous model, the direct effect of trait mindfulness on depressive symptoms was significant, such that higher levels of trait mindfulness predicted lower levels of depressive symptoms among participants $(\mathrm{B}=-.34, t=-9.13, p<.01)$. 
An examination of indirect effects revealed that the overall multiple mediation model was significant, $F(3,364)=103.11, p<.01$, accounting for $45.49 \%$ of the variance in depressive symptoms. An analysis of specific indirect pathways indicated that mastery significantly mediated the relationship between trait mindfulness and depressive symptoms. Higher trait mindfulness predicted greater feelings of mastery, which in turn predicted a decrease in depressive symptoms reported by participants $(\mathrm{CI}=$ $-.23,-.12)$. The indirect effect involving self-esteem was non-significant, suggesting that self-esteem is not a mediator in the relationship between mindfulness and depressive symptoms $(\mathrm{CI}=-.01, .003, n s)$ (see Figure 1.5).

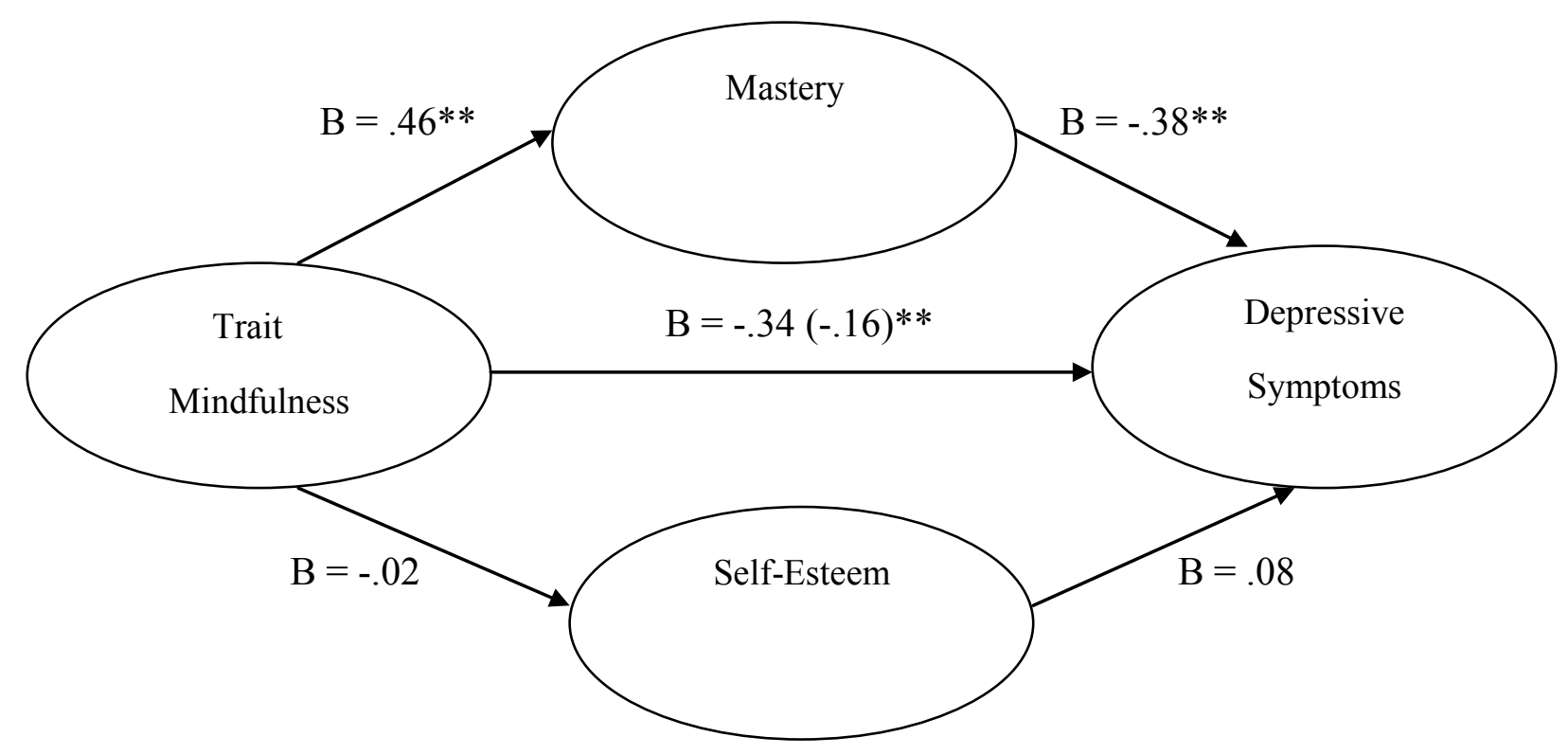

Figure 1.5. Self-appraisals (mastery, self-esteem) as multiple mediators in the relationship between trait mindfulness and depressive symptoms $(* p<.05$, $* * p<.01)$. 
Situation appraisals. A second multiple mediation model was analyzed to determine whether various aspects of stressor appraisals (i.e., appraisals of threat, distress, and control over potential stressors) mediated the relationship between trait mindfulness and depressive symptoms. Analysis of this model revealed that trait mindfulness scores positively predicted appraisals of control among participants $(\mathrm{B}=.03$, $t=4.29, p<.01)$, and negatively predicted appraisals of threat $(\mathrm{B}=-.03, t=-3.96, p<$ $.01)$ and distress $(\mathrm{B}=-.02, t=-3.44, p<.01)$. Looking at the direct effects of the proposed mediators on depressive symptoms, appraisals of distress positively predicted depressive symptoms $(\mathrm{B}=1.69, t=3.78, p<.01)$, while appraisals of control negatively predicted BDI scores $(\mathrm{B}=-.63, t=-2.34, p=.02)$. Threat appraisals also negatively predicted depressive symptoms, although this effect was only marginally significant $(\mathrm{B}=$ $-.86, t=-1.90, p=.06)$. As in the previous models, the direct effect of trait mindfulness on depressive symptoms was significant, with higher levels of trait mindfulness predicting lower levels of depressive symptoms among participants $(\mathrm{B}=-.34, t=-9.13, p$ $<.01)$.

The model including all mediators was significant, $F(4,363)=28.60, p<.01$, accounting for $23.13 \%$ of the variance in depressive symptoms. An analysis of indirect effects revealed that distress appraisals and control appraisals both significantly mediated the relationship between trait mindfulness and depressive symptoms. Higher trait mindfulness predicted greater control appraisals and fewer distress appraisals, each of which in turn predicted a decrease in depressive symptoms (Control CI $=-.04,-.01$; Distress $\mathrm{CI}=-.08,-.01)$. Interestingly, although threat appraisals were only marginally predictive of depressive symptoms $(p=.06)$, the confidence interval for the mediated 
effect involving threat appraisals did not include zero $(\mathrm{CI}=.0009, .06)$, indicating that the indirect effect of threat appraisals on the relationship between mindfulness and depressive symptoms was significant (see Figure 1.6).

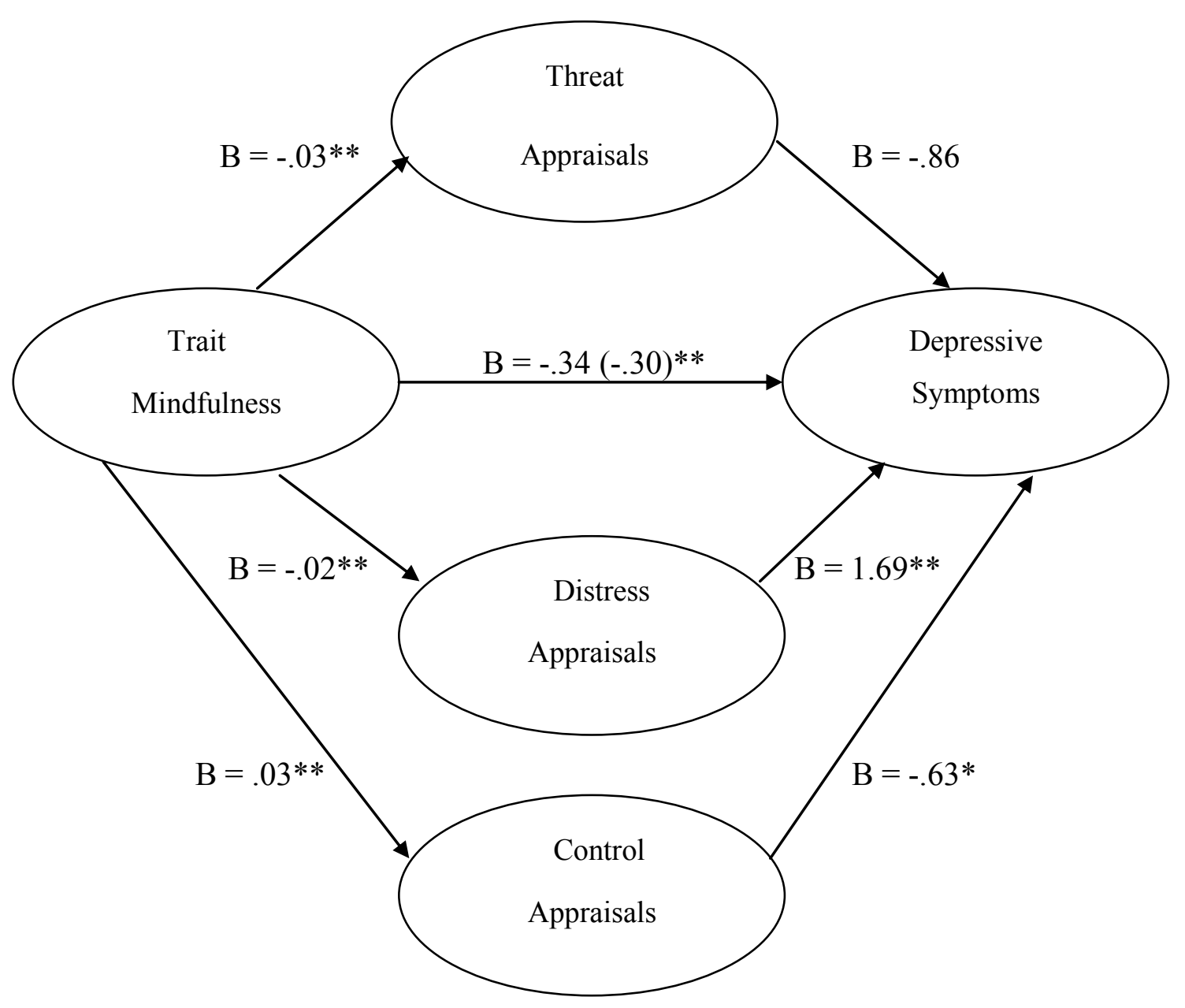

Figure 1.6. Situation appraisals (appraisals of threat, control, distress) as multiple mediators in the relationship between trait mindfulness and depressive symptoms $\left({ }^{*} p<\right.$ $.05, * * p<.01)$. 


\section{Discussion and Conclusions}

Previous research (Kabat-Zinn, 1990; Segal, Williams \& Teasdale, 2002) has posited that trait mindfulness promotes well-being by encouraging individuals to remain focused on the present moment, and that this present moment acceptance and attention may help to attenuate negative and emotional responses to stimuli that exacerbate depressive illness. Building upon this research, the purpose of the present investigation was to examine relationships between trait mindfulness, internalized stigma towards depressive symptoms, self and situation appraisals, and the endorsement of various types of coping strategies among young adults. It was hypothesized that trait mindfulness would discourage the use of emotion-focused coping strategies and the internalization of stigmas surrounding depression, while encouraging more adaptive appraisals of stressors and the endorsement of active (e.g., problem-focused) coping strategies.

In line with previous research (Beck et al., 1979; Lysaker, Roe \& Yanos, 2007; Moses, 2009; Rüsch, Corrigan, Todd \& Bodenhausen, 2010), internalized stigma and negative appraisals were highly predictive of depressive symptoms in the present study. Moderation analyses, however, indicated that the impact of stigma and appraisals on the development of depressive symptoms was weakened among highly mindful individuals. Mindful individuals appeared to be more resistant to internalizing stigmas towards depressive symptoms, and felt greater personal control over potentially stressful events, while simultaneously rating these events as less threatening and distressing compared to their less mindful counterparts. This finding is significant in light of the considerable research linking negative appraisals and internalized stigma to depressive symptoms (e.g., Beck, Rush, Shaw \& Emery, 1979, Moses, 2009; Rüsch et al., 2009; Teasdale, 
1983), and raises the possibility that the impact of these variables on depressive symptoms might be significantly lessened through the practice of mindful awareness.

Since trait mindfulness involves a tendency to attend to stimuli in the present moment (e.g., Kabat-Zinn, 1990, Weinstein, Brown \& Ryan, 2009), it was expected that mindfulness would encourage greater problem solving and the endorsement of problemfocused coping strategies. This relationship was supported in the present investigation. Specifically, trait mindfulness predicted greater endorsement of problem-focused coping strategies, which in turn predicted decreased depressive symptoms among young adults.

Dispositional mindfulness has also been associated with the ability to observe situations at arm's-length, allowing individuals to remain objective and to limit automatic, emotional responses to potentially stressful events (Carmody, 2009; Teasdale et al., 2002). As such, it was expected that mindfulness would inhibit the use of emotionfocused and avoidant coping strategies. This hypothesis was partially supported in the present study. As expected, trait mindfulness negatively predicted the endorsement of emotion-focused coping strategies among young adults, and this pathway predicted decreases in depressive symptomotology.

In regards to avoidant coping, however, multiple regression and mediation analyses indicated that trait mindfulness encouraged the use of avoidant coping strategies, which in turn predicted a reduction in depressive symptoms. Although the direction of this effect was not anticipated, highly mindful individuals are apt to attend to and process immediate reactions and sensations when faced with a stressor (rather than focusing upon the long-term resolution of stressors). Because of this tendency, coping 
strategies that allow for present-moment distraction from stressors and suspension of long-term planning might offer a reprieve from emotional distress elicited by these stressors, thereby helping to mitigate the development of depressive symptoms. Previous research (e.g., Suls \& Fletcher, 1985) has shown that avoidant coping strategies can be beneficial in the short term to alleviate distress and allow individuals time to adjust to new information or stimuli. This benefit might be especially apparent for highly mindful individuals who are habitually attuned to present-moment sensations, allowing them the time and space necessary to process thoughts, sensations and physical and emotional responses to stressors prior to acting upon them.

In addition, the wording of several items tapping avoidant coping in this study (i.e., "[I have] tried to keep my mind off things that are upsetting me", "[I have] tried to do things which I typically enjoy") suggest physical, cognitive or emotional distancing from a stressor. Since mindfulness fundamentally involves the ability to observe situations at arm's-length and accept thoughts and feelings without trying to change or control them (e.g., Kabat-Zinn, 1990; Segal, Williams \& Teasdale, 2002), it is possible that highly mindful individuals might endorse such coping items to the extent that they reflect acceptance of the situation at hand (rather than a desire to control or actively engage/change the situation), and a non-reactivity to stimuli (e.g., the suspension of automatic or reflexive emotional responding). Again, use of these types of strategies might help to alleviate emotional distress elicited by stressors, which subsequently contributes to well-being.

It was anticipated that trait mindfulness would also influence the ways in which individuals appraised potentially stressful situations. In particular, it was hypothesized 
that mindful individuals would tend to view situations more objectively than their less mindful peers, and that this objectivity would allow individuals to appraise events as less threatening and distressing relative to less mindful individuals. Moderated regression analyses indicated that distress appraisals were predictive of depressive symptoms, and higher levels of mindfulness did not appear to mitigate this effect. It does appear, however, that possessing low levels of trait mindfulness might exacerbate the relationship between distress appraisals and depressive symptoms. Notably, depressive symptoms were highest in the present study when individuals both appraised situations as being highly distressing and demonstrated low levels of trait mindfulness. It is possible, therefore, that individuals low in trait mindfulness might be especially vulnerable to developing symptoms of depression, particularly if they tend to view situations as highly distressing.

We recognize that the relationship between appraisals and depressive symptoms is likely bi-directional, in that negative appraisals might foster depressive symptoms, and symptomatology might then reinforce and perpetuate negative thinking and appraisal processes (Beck, Rush, Shaw \& Emery, 1979; Segal, Williams, Teasdale \& Gemar, 1996). It is possible, therefore, that reciprocal relationships exist between these variables, and that alternative models might be statistically and theoretically valid. The models explored in the present research were based primarily on research emphasizing negative appraisals as precursors to the development of depressive illness (Beck, 1967; Beck, Rush, Shaw \& Emery, 1979), and focus on the utility of identifying and targeting such appraisals in order to limit or prevent subsequent illness. 
Summarizing key results from this study, trait mindfulness appears to reduce depressive symptoms through enhancing feelings of mastery and problem-focused coping styles, and by reducing internalized stigma, the endorsement of emotion-focused coping strategies, and appraisals of distress in response to potentially stressful situations. Furthermore, for individuals who do tend to appraise situations as threatening and distressing, possessing higher levels of trait mindfulness appears to weaken the likelihood that these tendencies will culminate in depressive symptomatology. Given these findings, it is of further interest to examine how trait mindfulness influences the selection of specific coping strategies across different domains of functioning (Study 2).

\section{STUDY 2}

As outlined previously, trait mindfulness may promote the use of more active, problem-focused coping strategies by encouraging individuals to remain aware of their surroundings and their responses to events (e.g., Teasdale, Segal, Williams, Ridgeway, Soulsby \& Lau, 2000). In addition, by keeping potentially stressful events in perspective and limiting automatic, emotional responding, mindful individuals might feel more empowered or confident in their ability to cope with stressors. It remains possible, however, that while individuals may indicate high levels of mindfulness on self-report measures, this may not translate to mindful action in daily life. When faced with a stressor, for instance, self-reported 'mindful' individuals might respond in emotional or non-mindful ways, and the intention (however strong) to cope with events mindfully might be abandoned in favour of more familiar or convenient coping strategies (e.g., emotional expression, avoidance). Indeed, research in diverse areas, including goal motivation (Mesters, 2009) and addictions research (e.g., Dimeff \& Marlatt, 1998; 
McKay, 2009) has found that positive intentions frequently fail to predict spontaneous decision making and behaviours, and that behaviours may vary considerably across different situations.

Despite the possibility of a divergence between intentions and behaviours, to our knowledge, no research to date has been reported concerning the concordance between self-reported trait mindfulness and the use of mindful behaviour during periods of acute stress (e.g., the use of mindful coping strategies). As such, the purpose of Study 2 was to examine how mindful coping efforts vary, both across individuals and across different types of stressful situations. Specifically, Study 2 examined 1) the extent to which individuals endorsed mindfulness as a coping strategy, 2) the concordance between trait mindfulness (i.e., scores on the MAAS) and the endorsement of mindful coping strategies, 3) how well mindful coping predicted well-being over and above other types of coping strategies, and 4) whether the use of mindful coping varies across different situations or types of stressors. It was hypothesized that trait mindfulness would be highly correlated with mindful coping, and that both trait mindfulness and mindful coping efforts would be negatively associated with depressive symptoms, internalized stigma, and the use of emotion-focused coping strategies (e.g., rumination, self-blame). It was further hypothesized that these coping strategies would be associated with 1) reductions in subsequent appraisals of threat, 2) increased perceptions of control over stressful situations, and 3) more positive outcome appraisals across both student and community samples. 


\section{Method}

\section{Participants}

University sample. A primary sample of 372 male $(n=76 ; 20.4 \%)$ and female ( $n=296 ; 79.6 \%)$ students were recruited from the undergraduate student pool at Carleton University and registered to participate in an online study regarding individual difference variables (i.e., mindfulness, mastery, self-esteem), appraisals and coping strategies (see Appendix E for student and community recruitment notices). Participants ranged in age from 18-59 years $\left(M_{\text {age }}=21.08\right.$ years, $S D=5.74$ years $)$. Participants were predominantly White/Caucasian $(n=289 ; 77.7 \%)$, Asian $(n=26 ; 6.9 \%)$, Arab/Middle Eastern $(n=14 ; 3.8 \%)$, Black $(n=13 ; 3.5 \%)$ and South Asian $(n=10 ; 2.7 \%)$, with remaining participants reporting Aboriginal $(n=7 ; 1.9 \%)$, Latin American/Hispanic $(n=$ $3 ; 0.8 \%)$ or "Other" ethnicity $(n=10 ; 2.7 \%)$. Most students were registered on a fulltime basis $(n=333 ; 89.5 \%)$, with a small number of students reporting part-time $(n=22$; $5.9 \%)$ or special student status $(n=13 ; 3.5 \%)$.

Community sample. In order to extend upon and replicate previous research, data were collected from a second sample of community participants. A total of 275 adults ( $n=50$ male; $n=225$ female) responded to community advertising and completed this online study. Community participants ranged from $18-70$ years of age $\left(M_{\text {age }}=33.91\right.$ years, $S D=12.05$ years). A large majority of participants were White/Caucasian $(n=$ 190; 69.1\%), Asian $(n=41 ; 14.9 \%)$, South Asian $(n=21 ; 7.6 \%)$ and Arab/Middle Eastern $(n=7 ; 2.5 \%)$, with smaller numbers of participants indicating Aboriginal $(n=5$; $1.8 \%)$, Black $(n=3,1.1 \%)$, Latin American/Hispanic $(n=3,1.1 \%)$ or 'Other' $(n=5$; $1.8 \%$ ) ethnicity. Most participants reported being married or living in a common-law 
relationship ( $n=134 ; 48.7 \%$ ). Remaining participants were single/never married ( $n=$ $125 ; 45.5 \%)$, divorced/separated $(n=15,5.4 \%)$, or widowed $(n=1,0.4 \%)$. The majority of participants were University educated, either at the graduate $(n=104 ; 37.8 \%)$ or undergraduate level $(n=89 ; 32.4 \%)$. Other participants reported completing a college program $(n=47 ; 17.1 \%)$, high school $(n=7 ; 2.5 \%)$, or elementary school $(n=1 ; 0.4 \%)$. Twenty-seven participants (9.8\%) declined to report their highest level of education achieved.

\section{Procedure}

Student sample. Registration for this study was strictly voluntary, and conducted via the SONA electronic sign-up system at Carleton University. Students signing up for this study were provided with a link and invited to participate in an online study, which took approximately 60 minutes to complete. Upon accessing the online study, participants were directed to the informed consent form (see Appendix F). After providing consent, participants were then asked to complete a number of online questionnaires (see Appendix G), assessing demographic information, self-esteem (RSES; Rosenberg, 1965), mastery (RWB-M; Ryff, 1989), depressive symptoms (BDI; Beck, 1972), self-stigma for depressive symptoms (ISMIS; Ritsher, Otilingam \& Grajales, 2003), general coping styles (SCOPE; Matheson \& Anisman, 2003), domainspecific/situational coping strategies (modified SCOPE; Matheson \& Anisman, 2003), trait mindfulness (MAAS; Brown \& Ryan, 2003), and appraisals of various situations (AASQ; Kelly, Matheson \& Anisman, 2003).

In order to assess the use of mindful coping strategies, we embedded 8 items pertaining to mindfulness into the original SCOPE. These items are designed to assess 
various theorized components of mindfulness, including awareness (e.g., "[I have] become aware of my thoughts and feelings, without trying to change them or get carried away by them"), non-judgment (e.g., "[I have] paid attention to how I was reacting to the situation, without judging myself"), present-centered attention (e.g., "[I have] focused on the present moment rather than the past or future"), and acceptance (e.g., "[I have] made an effort to accept my feelings and thoughts for what they were, rather than trying to control them").

In addition, we included a shortened (32-item) version of the SCOPE, designed to assess coping in response to three specific types of stressors; financial, academic/workplace and interpersonal. The wording of items in the shortened version was identical to the wording used in the full version, however, the instructions were modified to reflect coping responses across these specific domains. For each type of stressor, participants were asked to think of a recent stressful event, and to identify the specific coping strategies they used in response to this situation. This modification allowed us to ascertain not only general coping styles, but also the specific coping strategies individuals use in response to specific types of events. This distinction was expected to further help to elucidate whether use of mindful coping varies across different types of potentially stressful situations.

Following completion of the questionnaire package, participants were directed to an online debriefing sheet and provided with contact information for local support services (see Appendix H). Students participating in this study received $0.5 \%$ toward their final grade in an introductory psychology class. 
Community sample. In order to observe the relationship between trait mindfulness and mindful coping efforts among a broader, representative sample of adults, community members were invited to participate in a second wave of this research. A description of this study and recruitment notices with URL were distributed to local businesses in Ottawa (e.g., coffee shops, bookstores), and notices were placed on public bulletin boards at community centres, consenting vendors and grocery stores. With the exception of slight changes in wording (e.g., references to "work" rather than "school") to reflect the target sample, community participants completed the same questionnaire package as student participants. Eligible community members received a \$5.00 CAD Tim Horton's gift certificate in exchange for completing this study.

\section{Measures}

All participants were asked to complete a battery of online questionnaires assessing demographic information, self-esteem, mastery, trait mindfulness, appraisals, depressive symptoms, and self-stigma for depressive symptoms (see Study 1 for description of measures and scoring). In addition, participants were asked to complete questionnaires assessing their endorsement of general coping styles, and use of specific coping strategies in response to a range of potential stressors. Internal consistency reliability was adequate to good for all measures across both student and community samples (see Table 2.1 for a summary of reliability coefficients).

General coping styles. The full 50-item Survey of Coping Profiles Endorsed (SCOPE; Matheson \& Anisman 2003) was used to assess general coping styles employed by participants. Items were rated on a 5-point Likert scale, ranging from 0 ('Never') to 4 ('Almost Always'). Mean scores were calculated for each of the 14 hypothesized coping 
dimensions (e.g., the 13 traditional coping dimensions described in Study 1, and the newly added "Mindful Coping" dimension). A Confirmatory Factor Analysis was used to confirm the factor structure of this measure (see results of CFA below).

Domain-specific/situational coping strategies. An abbreviated 32-item version of the SCOPE (SCOPE; Matheson \& Anisman, 2003) was used to assess participants' use of coping strategies in response to three specific types of stressors: Interpersonal, Financial, and Work/Academic. Participants were asked to think of a recent stressful situation in each of these domains (e.g., "Please take a moment to think of a recent personal stress that you have experienced (e.g., an argument with a friend or partner, etc.), and to indicate the extent to which they have used various coping strategies to resolve or deal with this stressor. Items were rated and scored in accordance with procedures described in Study 1. 
Table 2.1

Summary of Internal Consistency Coefficients for Student $(N=372)$ and Community $(N$ =275) Samples

\begin{tabular}{llcc}
\hline \multicolumn{1}{c}{ Measure } & \multicolumn{1}{c}{ Subscale(s) } & Student $\boldsymbol{\alpha}$ & Community $\boldsymbol{\alpha}$ \\
\hline Trait Mindfulness (MAAS) & - & .86 & .87 \\
Self-esteem & - & .79 & .77 \\
Mastery & - & .90 & .93 \\
Depressive Symptoms & - & .92 & .92 \\
Internalized Stigma & - & .89 & .91 \\
Appraisal & Threat & .94 & .94 \\
& Distress & .94 & .93 \\
& Control & .92 & .92 \\
Coping & Outcome & .89 & .91 \\
& Problem-Focused & .88 & .87 \\
& Emotion-Focused & .91 & .91 \\
& Avoidant & .66 & .71 \\
\hline
\end{tabular}

\section{Results}

\section{Preliminary Analysis}

Validity checks. Validity checks were performed to ensure integrity of online responses. Specifically, 7 pairs of questions were included across various questionnaires that were either repeated or nearly identical in content. Participants had to be within a two point range on at least 5 of 7 similar item pairs in order for the data to be considered valid. As this study was offered only to Canadian residents, an additional question was 
inserted ("Select \#4 if you currently live in Canada") to ensure that participants met demographic criteria for this study, and were reading the questions thoroughly and responding in good faith.

\section{Data Screening and Preliminary Considerations for Confirmatory Factor Analysis}

In order to confirm the theorized factor structure of the SCOPE, and to identify whether mindful coping represented a unique form of coping above and beyond previously supported and generally recognized coping dimensions, a Confirmatory Factor Analysis was conducted on our primary student sample data. Prior to conducting this analysis, responses to items on the SCOPE were examined for accuracy and missing or outlying values. Basic assumptions for CFA, including normality and factorability of data were also assessed.

Data were first evaluated in terms of sample size. The current sample size of $N=$ 372 was considered adequate for this type of analysis, according to recommendations by Comrey and Lee (1992; cited in Tabachnick \& Fidell). Data were screened for accuracy of entry and missing values using descriptive statistics. All observed scores of the SCOPE were within range (possible range of $0-4$ ), suggesting that there were no substantial data entry errors. No participants had missing values on the SCOPE.

To evaluate the shape of the distribution of variables, histograms were requested and skew and kurtosis were assessed for each coping dimension. One dimension (religiosity) showed a significant positive skew $(s k=7.97)$, while problem-solving $(s k=$ 3.53) and passive resignation $(s k=-3.31)$ showed evidence of negative skew.

Kolmogorov-Smirnov tests were significant $(p<.01)$, indicating that variables were not normally distributed. Linearity was then assessed through bivariate scatterplots for the 
sub-set of variables showing the greatest departure from normality. These scatterplots indicated adequate linearity between variables. Since the goal of the present study was to examine its underlying structure of the SCOPE, a decision was made not to delete variables or to pursue transformations of the data to improve normality.

\section{Outliers Among Cases}

Univariate outliers. Histograms, boxplots and stem and leaf plots were examined for all coping dimensions to identify univariate outliers, and $z$-scores were calculated to statistically confirm outlying cases. No highly influential cases were apparent upon a visual inspection of the data. This was further supported by an examination of $z$-scores (no $z$-scores exceeded 3.09 for any coping dimensions).

Multivariate outliers. After assessing univariate outliers, Mahalanobis distance was calculated to identify multivariate outliers. All obtained Mahalanobis values were compared against a $\chi^{2}$ critical value of $37.70,(d f=15, \alpha=.001)$. Four cases were identified as multivariate outliers according to this criterion ${ }^{2}$. Since the sample size is sufficient for conducting CFA and multivariate outliers may unduly distort the solution (Tabachnick \& Fidell, 2007), a decision was made to delete multivariate outliers and conduct further analyses on the remaining 368 cases $^{3}$.

A visual inspection of the correlation matrix for variables indicated that multicollinearity/ singularity was not present in the current data set (no bivariate correlations exceeded $r=.65$ ). The determinant of the matrix was also less than .00001 ,

${ }^{2}$ Case $97(\mathrm{MAH}=45.70)$, case $212(\mathrm{MAH}=47.99)$, case $362(\mathrm{MAH}=38.32)$, and case $372(\mathrm{MAH}=45.82)$.

3 CFA was run with and without multivariate outliers to substantiate this decision. The preservation of outlying cases resulted in a poorer fit of data to the model. Based on these results, it appeared that the retention of multivariate outliers may have weakened the overall stability of the solution. 
providing an additional indication that multicollinearity was not present among variables. Several bivariate correlations were significant with magnitudes exceeding $r=$ .30 , and Bartlett's test of sphericity was significant in the present sample, $\chi^{2}(1653)=$ $12044.92, p<.01$, suggesting that the data may be subjected to factor analysis.

\section{Confirmatory Factor Analysis}

Previous research on stress and coping (e.g., Carver, Scheier \& Weintraub, 1989; Folkman, Lazarus, Dunkel-Schetter, DeLongis, Gruen \& Rand, 1986) has provided strong theoretical and empirical support for the existence of three primary coping dimensions: problem-focused coping, which encompasses the use of strategies aimed at resolving the source of a stressor (e.g., cognitive restructuring, problem-solving, social support seeking); emotion-focused coping, including strategies aimed at reducing one's emotional responses to a stressor (e.g., emotional expression and/or emotional containment, rumination, wishful thinking); and avoidant coping, including strategies aimed at physical or cognitive distraction from a stressor (e.g., engagement in other activities). Based upon this literature, a series of Confirmatory Factor Analyses (CFA) were conducted using LISREL v. 8.80 Structural Equation Modeling software in order to:

1) determine how well the original 50-items comprising the SCOPE load on to previously established/ theorized coping dimensions,

2) examine how well our newly created mindful coping items load on a theorized "mindful coping" dimension, and 
3) compare the base (50-item) model to a larger model including mindful coping items in order to identify if mindful coping represents a unique coping construct, and to determine which solution provided a better fit for the data.

SCOPE 50-item model. Bivariate correlations were first examined to determine the strength and direction of the relationships between SCOPE subscales for each of the three primary theoretical coping dimensions (see Table 2.2 for a summary of bivariate correlations). A Confirmatory Factor Analysis of the 50-item SCOPE was then conducted on our larger, primary student sample $(N=368)$. Based on the underlying theoretical structure of this measure, a three-factor model was examined (corresponding to the three overarching subscales of the SCOPE: problem-focused, emotion-focused and avoidant coping). Parameters were estimated using maximum likelihood (ML) estimation. Four goodness-of-fit indices were used to evaluate the fit of the model: Chisquare $\left(\chi^{2}\right)$, the Comparative Fit Index (CFI), the Standardized Root Mean Square Residual (SRMR), and the Root Mean Square Error of Approximation (RMSEA). It has been argued that the $\chi^{2}$ statistic is overly sensitive when sample sizes are large and/or several variables are modeled (Kline, 1998; Saris, Satorra \& van der Veld, 2009). As such, a $\chi^{2} / d f$ ratio was used to account for the number of parameters being modeled, and to provide a more robust estimate of fit (Kline, 1998). Criteria for acceptable fit were set at $\chi^{2} / d f<3.00$ (Jöreskog, 1969; Schumacker \& Lomax, 2004; Wheaton, Muthen, Alwin $\&$ Summers, 1977), CFI $>.90$ (Ullman, 2007), SRMR $\leq .08$, and RMSEA of $<.08$, with 90\% confidence intervals ranging from .06 to .08 (Hu \& Bentler, 1999).

This analysis indicated that items pertaining to religion (e.g., "[I have] turned to God") and humour (e.g., "[I have] told humorous stories") did not strongly load on any 
factors. As such, these items were deleted in order to improve the overall fit of the coping model. The 'Wishful thinking' subscale did not appear to be well defined by its items in the present sample; however, as expected, these items significantly loaded on the higher-order emotion-focused coping construct. Factor loadings and fit indices for the CFA are presented in Table 2.3. All goodness-of fit indices revealed that the three-factor model fit the data well, $\chi^{2} / d f=2.38, p<.01, \mathrm{CFI}=.94, \mathrm{SRMR}=.08, \mathrm{RMSEA}=.064$ $(90 \% \mathrm{CI}=.061 ; .068)$ 
Table 2.2

Summary of Bivariate Correlations, Means and Standard Deviations for SCOPE Subscales.

\begin{tabular}{|c|c|c|c|c|c|c|c|c|c|c|c|c|}
\hline Coping Subscale & 1 & 2 & 3 & 4 & 5 & 6 & 7 & 8 & 9 & 10 & 11 & 12 \\
\hline 1. Problem-Solving & -- & & & & & & & & & & & \\
\hline 2. Cognitive Restructuring & $.59 * *$ & -- & & & & & & & & & & \\
\hline 3. Social Support Seeking & $.60 * *$ & $.33^{* *}$ & -- & & & & & & & & & \\
\hline 4. Rumination & $.15^{* *}$ & $.21 * *$ & $.22 * *$ & -- & & & & & & & & \\
\hline 5. Emotional Expression & .07 & $.15^{* *}$ & $.27 * *$ & $.64 * *$ & -- & & & & & & & \\
\hline 6. Emotional Containment & -.07 & $.21 * *$ & $-.18 * *$ & $.42 * *$ & $.42 * *$ & -- & & & & & & \\
\hline 7. Self-Blame & $.15^{* *}$ & $.33 * *$ & $.11 *$ & $.56^{* *}$ & $.43 * *$ & $.41 * *$ & -- & & & & & \\
\hline 8. Other-Blame & $.14^{* *}$ & $.20^{* *}$ & $.20 * *$ & $.36^{* *}$ & $.42 * *$ & $.26^{* *}$ & $.34 * *$ & -- & & & & \\
\hline 9. Passive Resignation & $.14 * *$ & $.29 * *$ & $.18^{* *}$ & $.48 * *$ & $.46^{* *}$ & $.36^{* *}$ & $.47 * *$ & $.40 * *$ & -- & & & \\
\hline 10. Wishful Thinking & $.12^{*}$ & $.33^{* *}$ & $.13^{*}$ & $.63 * *$ & $.52 * *$ & $.51 * *$ & $.49 * *$ & $.43 * *$ & $.48 * *$ & -- & & \\
\hline 11. Active Distraction & $.37^{* *}$ & $.37 * *$ & $.28^{* *}$ & -.01 & -.05 & -.02 & .03 & .10 & .04 & .05 & -- & \\
\hline 12. Avoidance & $.25^{* *}$ & $.29 * *$ & $.13^{*}$ & .10 & $.17 * *$ & $.26 * *$ & .10 & $.15^{* *}$ & $.17 * *$ & $.25^{* *}$ & $.26^{* *}$ & -- \\
\hline Mean & 2.28 & 2.37 & 2.02 & 2.40 & 1.28 & 2.03 & 2.24 & 1.05 & 1.53 & 2.48 & 2.34 & 1.96 \\
\hline SD & .79 & .78 & 1.19 & 1.09 & 1.03 & 1.15 & 1.17 & 1.09 & .96 & 1.04 & .65 & .83 \\
\hline
\end{tabular}

${ }^{*} p<.05, * * p<.01$ 
Table 2.3

Summary of Confirmatory Factor Analysis for SCOPE Subscales

Subscale Loading on Higher-order Latent Factor(s)

\begin{tabular}{cccc}
\hline & $\begin{array}{c}\text { Item } \\
\text { Loading }\end{array}$ & $\begin{array}{c}\text { Problem- } \\
\text { Focused }\end{array}$ & $\begin{array}{c}\text { Emotion- } \\
\text { Focused }\end{array}$
\end{tabular} Avoidant

Problem-solving

1.78

Sought information to resolve problems

Made plans to overcome my problems .38

Taken action on problems

Taken steps to overcome my problems

.39

Cognitive Restructuring

1.45

Told myself others have dealt with problems like mine

Decided I can grow and learn through my problems

Told myself others have problems like mine

Looked for how I can learn from situation

\section{Social Support Seeking}

0.70

Talked with friends or relatives $\quad .78$

Sought advice of others $\quad .80$

Asked others for help $\quad .76$

Sought reassurance/support from others

Told others I was depressed or upset

\section{Rumination}

Gone over problems over and over

Thought about my problems a lot

Worried about my problems a lot 


$\begin{array}{cccc}\text { Item } & \begin{array}{c}\text { Problem- } \\ \text { Focused }\end{array} & \begin{array}{c}\text { Emotion- } \\ \text { Focused }\end{array} & \text { Avoidant }\end{array}$

Emotional Expression

1.35

Been very emotional compared to usual

Been very emotional in how I react

Found myself crying more than usual

Emotional Containment

Kept my feelings bottled up inside

Tried to act as if I wasn't upset

Held in my feelings

Tried to act as if I wasn't feeling bad

.86

Other Blame

0.66

Blamed others for creating problems

Thought about who is responsible for my problems

Thought about how problems have been caused by others

\section{Self Blame}

Blamed myself for my problems

Thought about how I have brought problems on myself

Decided problems are a result of past actions

\section{Passive Resignation}

Accepted there is nothing I can do to change my situation

Tried to take whatever came my way

Decided to wait and see how things

turn out

Resigned myself to my problems 


$\begin{array}{cccc}\text { Item } & \begin{array}{c}\text { Problem- } \\ \text { Focused }\end{array} & \begin{array}{c}\text { Emotion- } \\ \text { Focused }\end{array} & \text { Avoidant }\end{array}$

\section{Wishful Thinking}

4.11

Fantasized/thought about unreal things $\quad .14$

Wished problem would go away $\quad .19$

Daydreamed about how things may turn out

Wished I was better at dealing with problems

Physical Distraction

Tried to do things I typically enjoy

Exercised

Become involved in recreation/

pleasure activities

Gone out

.35

Cognitive Distraction (Avoidance)

Tried to keep my mind off things

Tried to distract myself from problems

.75

Avoided thinking about my problems

.46

\begin{tabular}{lc}
\hline Fit Indices & \\
$\chi^{2}$ & 2109.91 \\
$\mathrm{df}$ & 887 \\
$\chi^{2} / \mathrm{df}$ & 2.38 \\
$\mathrm{CFI}$ & 0.94 \\
SRMR & 0.08 \\
RMSEA & 0.06
\end{tabular}


SCOPE mindful coping subscale. A second Confirmatory Factor Analysis was conducted to determine how well the newly created mindful coping items loaded on a theorized mindful coping construct. As in the previous analysis, bivariate correlations were examined to determine the strength and direction of the relationships between mindful coping items (see Table 2.4). A Confirmatory Factor Analysis was then conducted using maximum likelihood estimation. A preliminary item analysis of mindful coping items revealed that one of the eight newly created items ("I...focused on the present moment, rather than the past or future") did not load strongly on the mindful coping factor. Accordingly, this item was excluded from further analyses to improve model fit.

An examination of fit indices indicated that the mindful coping model fit the data well, $\chi^{2}(13)=20.91, p=.08, \mathrm{CFI}=.098, \mathrm{SRMR}=.03, \mathrm{RMSEA}=.04(90 \% \mathrm{CI}=.041$; .073 ). All items significantly loaded ( $\geq 0.5$ ) on the mindful coping factor (see Table 2.5). 
Table 2.4

Summary of Bivariate Correlations, Means and Standard Deviations for Mindful Coping Items

\begin{tabular}{|c|c|c|c|c|c|c|c|c|}
\hline Mindful Coping Item & 1 & 2 & 3 & 4 & 5 & 6 & 7 & 8 \\
\hline 1. Focused on present moment & -- & & & & & & & \\
\hline 2. Paid attention to reactions & $.34 * *$ & -- & & & & & & \\
\hline 3. Observed thoughts & $.19^{* *}$ & $.39 * *$ & -- & & & & & \\
\hline 4. Breathe and focus on present & $.26 * *$ & $.32 * *$ & $.25 * *$ & -- & & & & \\
\hline 5. Accept thoughts and feelings & $.12 *$ & $.32 * *$ & $.40 * *$ & $.23 * *$ & -- & & & \\
\hline 6. Taken time to breathe and calm down & $.17^{*}$ & $.31 * *$ & $.22 * *$ & $.51 * *$ & $.27 * *$ & -- & & \\
\hline 7. Awareness of thoughts and feelings & $.13 *$ & $.34 * *$ & $.42 * *$ & $.30 * *$ & $.38 * *$ & $.31 * *$ & -- & \\
\hline 8. Keep situation in perspective & $.21 * *$ & $.38 * *$ & $.30 * *$ & $.34 * *$ & $.30 * *$ & $.33 * *$ & $.35^{* *}$ & -- \\
\hline Mean & 2.45 & 1.90 & 1.95 & 2.42 & 2.01 & 2.08 & 2.03 & 2.49 \\
\hline SD & .99 & 1.04 & .98 & 1.07 & .96 & 1.04 & .95 & .93 \\
\hline
\end{tabular}

${ }^{*} p<.05, * * p<.01$ 
Table 2.5

Summary of Confirmatory Factor Analysis for Mindful Coping Items

\begin{tabular}{lc}
\hline Item & $\begin{array}{c}\text { Item Loading on } \\
\text { Mindful Coping } \\
\text { Factor }\end{array}$ \\
\hline Paid attention to reactions & 0.63 \\
Observed thoughts & 0.61 \\
Breathe and focus on present & 0.51 \\
Accept thoughts and feelings & 0.55 \\
Taken time to breathe and calm down & 0.50 \\
Awareness of thoughts and feelings & 0.60 \\
Keep situation in perspective & 0.53 \\
\hline Fit Indices & 0.03 \\
$\chi^{2}$ & 0.91 \\
df & 0.93 \\
CFI & 13 \\
\hline
\end{tabular}

\section{Model Comparison}

In order to determine which model provided the best fit for the data, fit indices for the original 50-item SCOPE were compared against a full (57-item) model including the mindful coping factor. An analysis of Comparative Fit Indices, SRMR and RMSEA indicated that both models acceptably fit the data. According to recommendations by 
Burnham and Anderson (2002), second-order Akaike Information Criterion (CAIC) ${ }^{4}$ values were compared to estimate which of these two candidate models best minimized loss of information from the 'true' theorized model, and thus provided the best fit for the data (Akaike, 1974; Burnham \& Anderson, 2002). This value was smaller for the original three-factor model than for the larger model, indicating a slightly better fit for the more parsimonious model.

Chi-squared model fitting was then conducted, using procedures described by Wilson and Hilferty (1931). Differences in degrees of freedom were considered along with differences in CAIC values between the two models. This analysis was nonsignificant, $\chi_{\text {diff }}^{2}(314)=625.47, p<.01$, indicating that the degree of fit was comparable between the two models (see Table 2.6 for a summary of CFA).

Although previous research has suggested that coping responses are not mutually exclusive (Folkman et al., 1986) and moderate correlations were observed between coping subscales in this investigation, results of the present analysis indicate that emotion-focused, avoidant, problem-focused and mindful coping constructs possess distinct factor structures. Confirmatory Factor Analysis suggested that although the theoretically driven four-factor structure (Problem-Focused, Emotion-Focused, Avoidant and Mindful Coping) did not significantly improve upon the more parsimonious threefactor model, it remained an acceptable fit for the data, and all four factors were well defined, with loadings exceeding .60. See Table 2.6 for a summary of comparative statistics.

\footnotetext{
${ }^{4}$ Burnham and Anderson (2002) recommend interpreting second-order CAIC values rather than Model AICs, as CAICs include a correction for large sample sizes and the estimation of multiple parameters, thus minimizing the likelihood of over-fitting models.
} 
Table 2.6

Summary of Comparative Fit Statistics for Three and Four-Factor SCOPE Models

\begin{tabular}{lcc}
\hline Comparative Fit Indices & Model 1 & Model 2 \\
\hline CAIC & 2939.76 & 3632.26 \\
$\chi^{2}$ & 2109.91 & 2735.38 \\
df & 887 & 1201 \\
$\chi^{2} / \mathrm{df}$ & 2.38 & 2.28 \\
CFI & .94 & .94 \\
SRMR & .08 & .08 \\
RMSEA $(90 \% \mathrm{CI})$ & $.06(.061, .068)$ & $.04(0.041 ; 0.073)$ \\
\hline
\end{tabular}

\section{Multiple Regression Analyses}

Based upon previous research (e.g., Kabat-Zinn, 1990; Weinstein, Brown \& Ryan, 2009), a series of multiple regression analyses were conducted using both our student sample and a community sample to examine (and replicate) the relationship between trait mindfulness, depressive symptoms, internalized stigma toward depressive symptoms, appraisals and coping dimensions among undergraduate students and adult community members.

Bivariate correlations. Bivariate correlations were examined to assess the strength and direction of the relationship between trait mindfulness and coping dimensions supported by Confirmatory Factor Analyses (e.g., problem-focused, emotionfocused, avoidant and mindful coping), as well as the relationship between these coping dimensions and depressive symptoms, internalized stigma and appraisals of potentially 
stressful events. Consistent with previous research, trait mindfulness was negatively correlated with depressive symptoms (Herwig, Kaffenberger, Lutz \& Brühl, 2010), internalized stigma, appraisals of threat and distress (e.g., Herwig et al., 2010), and the use of emotion-focused coping strategies (Langer, 1989; Weinstein, Brown \& Ryan, 2009) across both student and community samples. Mindful coping was negatively correlated with depressive symptoms, and positively correlated with control appraisals across both samples. As expected, mindful coping was also negatively associated with appraisals of threat and distress, although these relationships failed to reach statistical significance. Surprisingly, mindful coping was not significantly correlated with scores on the MAAS, indicating a lack of concordance between mindful coping and trait mindfulness (as assessed by the MAAS) for either the student or community samples in the present study (see Table 2.7 for a summary of bivariate correlations). 
Table 2.7

Bivariate Correlations Between Trait Mindfulness (MAAS), Internalized Stigma (ISMIS), Depressive Symptoms (BDI), Appraisals, and Endorsement of Coping Dimensions Among Student $(N=372)$ and Community $(N=275)$ Samples

\begin{tabular}{|c|c|c|c|c|c|c|c|c|c|c|}
\hline & 1 & 2 & 3 & 4 & 5 & 6 & 7 & 8 & 9 & 10 \\
\hline 1. Trait Mindfulness (MAAS) & -- & $-.49 * *$ & $-.43 * *$ & -.01 & $-.42 * *$ & -.08 & -.08 & $-.13^{*}$ & $-.19 * *$ & .06 \\
\hline 2. Internalized Stigma & $-.47 * *$ & -- & $.59 * *$ & .03 & $.51 * *$ & .01 & -.01 & $.35 * *$ & $.35^{* *}$ & $-.15 * *$ \\
\hline 3. Depressive Symptoms & $-.48 * *$ & $.64 * *$ & -- & -.09 & $.60 * *$ & $-.11 *$ & $-.12 *$ & $.28 * *$ & $.29 * *$ & -.05 \\
\hline 4. Problem-focused coping & .08 & -.11 & $-.24 * *$ & -- & $.19 * *$ & $.43 * *$ & $.68 * *$ & .04 & .02 & .10 \\
\hline 5. Emotion-focused coping & $-.55 * *$ & $.54 * *$ & $.69 * *$ & -.09 & -- & $.18^{* *}$ & $.11^{*}$ & $.29 * *$ & $.33 * *$ & -.04 \\
\hline 6. Avoidant coping & .03 & -.09 & $-.17 * *$ & $.36 * *$ & .01 & -- & $.52 * *$ & .08 & .10 & .05 \\
\hline 7. Mindful coping & .04 & -.08 & $-.22 * *$ & $.62 * *$ & -.06 & $.44 * *$ & -- & -.10 & -.09 & $.16^{* *}$ \\
\hline 8. Distress Appraisals & $-.23 * *$ & $.36 * *$ & $.33 * *$ & -.09 & $.37 * *$ & .01 & $-.15^{*}$ & -- & $.90 * *$ & $-.25 * *$ \\
\hline 9. Threat Appraisals & $-.25 * *$ & $.36 * *$ & $.33 * *$ & -.07 & $.39 * *$ & .03 & -.12 & $.93 * *$ & -- & $-.24 * *$ \\
\hline 10. Control Appraisals & $.13 *$ & -.14 & $-.13 *$ & $.14^{*}$ & $-.14^{*}$ & .08 & $.11 *$ & $-.20 * *$ & $-.16 * *$ & -- \\
\hline
\end{tabular}

$* p<.05 ; * * p<.01$

N.B. Values above the diagonal represent student sample; values below the diagonal represent community sample. 


\section{Trait mindfulness, depressive symptoms, internalized stigma and coping.}

Consistent with relationships observed in Study 1, trait mindfulness negatively predicted depressive symptoms, $F(1,360)=83.66, p<.01, \mathrm{R}^{2}=.183 ; F(1,273)=80.26, p<.01$, $R^{2}=.224$, internalized stigma, $F(1,360)=114.18, p<.01, R^{2}=.237 ; F(1,273)=76.35$, $p<.01, R^{2}=.216$, and the endorsement of emotion-focused coping strategies, $F(1,360)=$ $76.89, p<.01, R^{2}=.174 ; F(1,360)=115.75, p<.01, R^{2}=.295$ among student and community participants in the present investigation, respectively.

It was hypothesized that trait mindfulness would predict the endorsement of mindful coping strategies among participants. To replicate findings from Study 1, we also hypothesized that trait mindfulness would negatively predict depressive symptoms, internalized stigma, and the use of emotion-focused coping strategies. Contrary to our expectation, trait mindfulness was not a significant predictor of mindful coping in either our student sample $(p=.07)$ or community sample $(p=.50)$, suggesting that high trait mindfulness did not encourage the deployment of mindfulness-based behaviours in response to stressors.

\section{Mindful Coping and Appraisal}

The concept of 'mindful coping' in this investigation was framed as the endorsement of behaviours (e.g., breathing) that focus on present-moment awareness and attention to physical and emotional responses to stressors. We theorized that the use of such strategies would allow individuals to remain grounded in the present moment, and to evaluate situations based upon information at hand, rather than responding emotionally based upon past experience or concern for future outcomes (e.g., Herwig et al., 2010; Kabat-Zinn, 1990). Specifically, we hypothesized that the deployment of these 
mindfulness techniques in response to stressors would negatively predict appraisals of threat and distress, and positively predict perceptions of control over potential stressors. As expected, mindful coping predicted greater appraisals of control, $F(1,357)=$ $9.00, p<.01, R^{2}=.022 ; F(1,273)=3.31, p=.07, R^{2}=.018$, and positive outcome expectancies, $F(1,357)=6.94, p<.01, R^{2}=.016 ; F(1,273)=11.15, p<.01, R^{2}=.036$ across student and community samples, respectively, and inversely predicted distress appraisals among participants, $F(1,357)=3.44, p=.05, R^{2}=.010 ; F(1,273)=5.91, p=$ $.02, R^{2}=.018$. Mindful coping was also negatively associated with threat appraisals, although this relationship did not reach statistical significance for the student sample $(p=$ $.09)$, and only approached significance for the community sample $(p=.06)$.

\section{Mindfulness and Situational Coping}

Results from Studies 1 and 2 indicate that, in general, trait mindfulness negatively predicts the endorsement of emotion-focused coping styles, and is positively associated with the endorsement of both problem-focused and avoidant coping styles. It remains unclear, however, if the type of coping strategies endorsed by individuals differs across different types of situations or stressors. A series of multiple regression analyses were conducted to determine if individuals employed problem-focused, emotion-focused, avoidant and mindful coping strategies differently in response to three common types of stressors: interpersonal (e.g., a conflict with a friend or partner), financial (e.g., delay in receiving expected paycheque or loan) and workplace/academic (e.g., threat to job security or academic standing).

Interpersonal stressors. Multiple regression analyses were used to identify specific coping strategies endorsed by individuals in response to interpersonal stressors, 
and in particular to determine if trait mindfulness predicted an increased use of mindful coping efforts in the context of interpersonal conflicts (e.g., argument with a friend or partner). Contrary to our expectation, trait mindfulness was not significantly associated with the use of mindful coping strategies $(p=.10 ; p=.96)$, avoidance of interpersonal stressors $(p=.36 ; p=.33)$, or problem-focused coping strategies $(p=.82 ; p=.14)$ in student or community samples, respectively. Trait mindfulness was, however, a significant predictor of emotion-focused coping in the present study. Consistent with results from Study 1, higher trait mindfulness scores predicted lower endorsement of emotion-focused coping responses among both student, $F(1,362)=52.29, p<.01, R^{2}=$ .126 and community participants, $F(1,273)=76.43, p<.01, R^{2}=.219$.

Financial stressors. A second series of multiple regression analyses were performed to determine if trait mindfulness predicted the use of specific coping techniques following a self-identified financial stressor (e.g., job loss, debt management, unexpected expenses). Although an overall effect of trait mindfulness on coping was not observed, gender differences emerged with respect to mindfulness and coping with financial stressors. Among women, trait mindfulness predicted decreases in emotionfocused coping strategies in response to financial stressors (student $F(1,288)=23.20, p<$ $.01, R^{2}=.075$; community $\left.F(1,223)=50.81, p<.01, R^{2}=.186\right)$. Interestingly, however, this relationship was not observed among men in either the student sample $(p=.07)$ or community sample $(p=.07)$. Among men, higher levels of trait mindfulness failed to predict the endorsement of any specific type of coping response following a financial stressor, suggesting that mindfulness did not significantly inform behaviours or cognitive/emotional responses to stressors in this context. 
Academic/work stressors. A final set of multiple regression analyses were examined to determine if trait mindfulness predicted differential use of coping strategies in response to a specific, self-identified academic or workplace stressor (e.g., receiving a failing grade on a test or exam, receiving a poor performance review or demotion at work, etc.). Again, these analyses revealed that individuals higher in trait mindfulness were less likely to report the use of emotion-focused coping strategies in response to academic or workplace stressors (student $F(1,362)=48.86, p<.01, R^{2}=.119$; community $\left.F(1,272)=52.03, p<.01, R^{2}=.161\right)$, but were not significantly more or less likely to employ other types of coping responses, including problem-solving $(p=.55 ; p=$ $.52)$, avoidant $(p=.36 ; p=.49)$ or mindfulness-based coping strategies $(p=.51 ; p=.25)$.

\section{Mediating Effects of Internalized Stigma and Coping Dimensions}

In order to replicate and extend the model supported in Study 1, mediation analyses were performed to determine if trait mindfulness influenced depressive symptoms indirectly through various coping pathways, and to determine if these pathways held among both student and community samples. Based upon previous research (e.g., Weinstein et al., 2009), it was hypothesized that trait mindfulness would negatively predict depressive symptoms in part by encouraging the use of active, problem-based coping strategies, and limiting the use of emotion-focused coping responses. We further predicted that trait mindfulness would limit self-judgment and the tendency of individuals to self-stigmatize for depressive symptoms, and that this acceptance would in turn predict reductions in symptomatology (e.g., Kabat-Zinn, 1990). As in Study 1, multiple mediation analyses were performed to test these hypotheses for 
both student and community samples, using Preacher and Hayes' (2008) macro for multiple mediation with 1000 iterations.

Student sample. As anticipated, trait mindfulness inversely predicted both internalized stigma $(\mathrm{B}=-.14, t=-6.93, p<.01)$ and the endorsement of emotion-focused coping strategies $(\mathrm{B}=-.20, t=-8.77, p<.01)$ among student participants, and was negatively but non-significantly associated with the endorsement of both avoidant $(\mathrm{B}=$ $.01, \mathrm{t}=-1.45, \mathrm{p}=.15)$ and problem-focused coping strategies among young adults $(\mathrm{B}=$ $.001, t=-.10, p=.92$ ). An examination of the pathways between our proposed mediators and outcome variable indicated that avoidant coping $(\mathrm{B}=-.96, t=-2.92, p<.01)$ negatively predicted depressive symptoms, while problem-focused coping marginally predicted lower symptomotology $(\mathrm{B}=-.42, t=-1.89, p=.06)$. As in previous studies, the endorsement of emotion-focused coping strategies $(\mathrm{B}=.82, t=9.72, p<.01)$ and internalized stigma $(\mathrm{B}=.97, t=6.93, p<.01)$ strongly and positively predicted depressive symptoms among participants. In line with our predictions, the endorsement of mindful coping strategies inversely predicted depressive symptoms, although this relationship did not reach statistical significance $(\mathrm{B}=-.28, t=-77, p=.44)$. The direct effect of trait mindfulness on depressive symptoms was also significant, suggesting that higher levels of trait mindfulness predicted lower levels of depressive symptoms among participants $(\mathrm{B}=-.39, t=-9.15, p<.01)$.

An examination of indirect effects revealed that the overall multiple mediation model was significant, $F(6,355)=64.73, p<.01$. The model including all mediators accounted for $51.44 \%$ of the variance in depressive symptoms. An analysis of individual parameters indicated that internalized stigma significantly mediated the relationship 
between trait mindfulness and depressive symptoms, such that higher trait mindfulness predicted reductions in internalized stigma, which in turn predicted a decrease in the prevalence and severity of depressive symptoms reported by participants $(\mathrm{CI}=-.19,-10)$. Similarly, emotion-focused coping (CI = -.21, -.11) significantly mediated the relationship between trait mindfulness and depressive symptoms. As hypothesized, the direction of these effects indicated that higher levels of mindfulness were associated with lower endorsement of emotion-focused coping strategies, which in turn predicted fewer reported depressive symptoms. Interestingly, the confidence intervals for problemfocused, avoidant and mindful coping all spanned zero, suggesting that the observed mediation effect in this model was driven by the suppressing influence of trait mindfulness on both internalized stigma and the endorsement of emotion-focused coping strategies (see Figure 2.1 for a summary of multiple mediation model). 


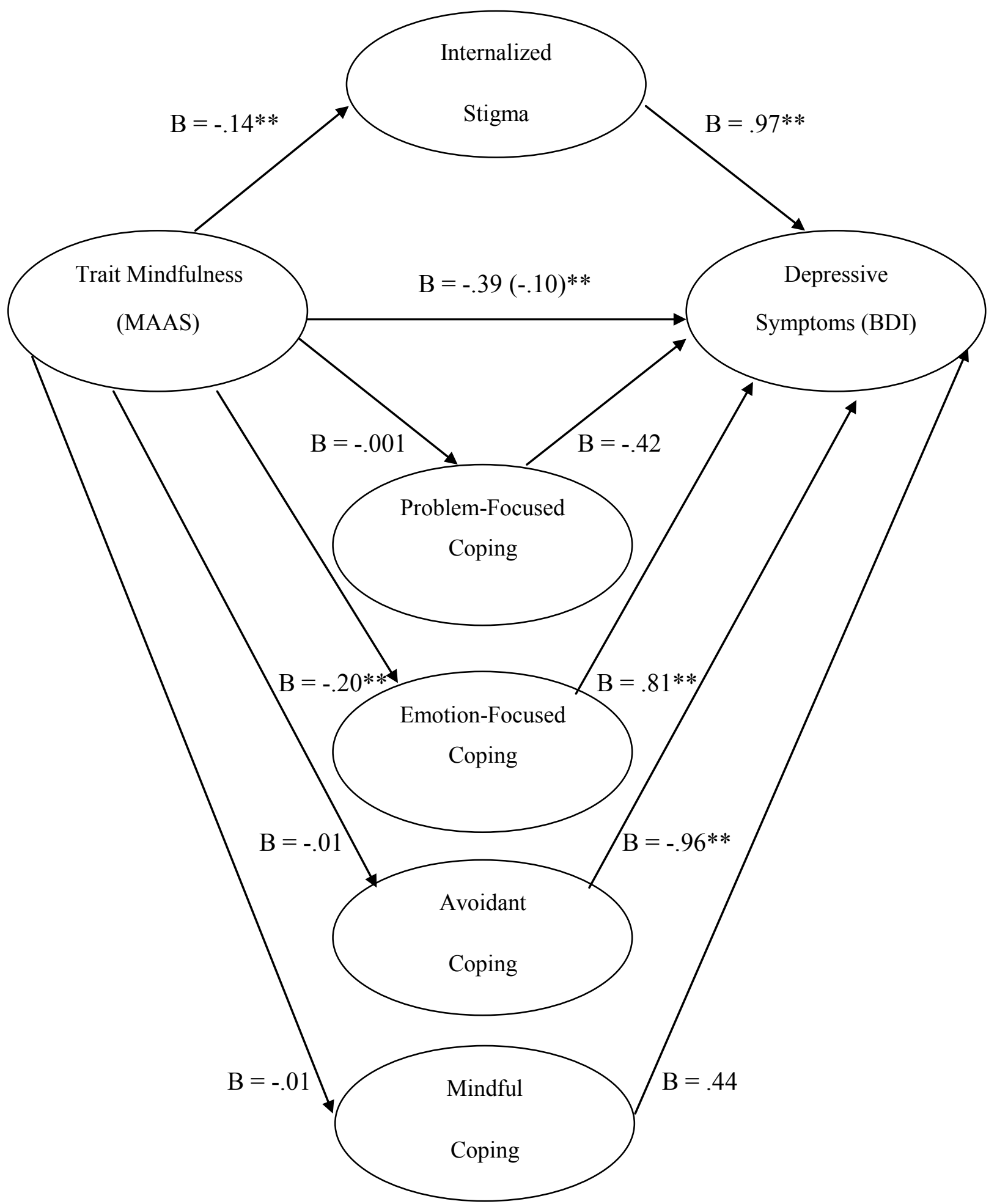

Figure 2.1. Internalized Stigma (ISMIS) and coping styles (problem-focused, emotionfocused, avoidant and mindful coping) as multiple mediators in the relationship between trait mindfulness and depressive symptoms among students $\left({ }^{*} p<.05,{ }^{*} p<.01\right)$. 
Community sample. A second multiple mediation analysis was performed to assess the relationship between trait mindfulness, coping and depressive symptoms among a community sample. As in the previous analysis, trait mindfulness inversely predicted both internalized stigma $(\mathrm{B}=-.13, t=-8.74, p<.01)$ and the endorsement of emotion-focused coping strategies $(\mathrm{B}=-.24, t=-10.76, p<.01)$ among participants. Trait mindfulness was positively but non-significantly associated with the endorsement of avoidant $(\mathrm{B}=.004, t=.57, p=.57)$, problem-focused $(\mathrm{B}=.01, t=1.26, p=.21)$, and mindful coping strategies among community participants $(\mathrm{B}=.001, t=.20, p=.84)$. Consistent with previous observations, the endorsement of emotion-focused coping strategies $(\mathrm{B}=.99, t=9.68, p<.01)$ and internalized stigma $(\mathrm{B}=1.10, t=7.26, p<.01)$ were highly predictive of depressive symptoms in the present sample, and mindful coping marginally predicted a decrease in depressive symptoms $(\mathrm{B}=-.78, t=-1.91, p=.06)$.

The direct effect of trait mindfulness on depressive symptoms was significant, suggesting that higher levels of trait mindfulness predicted lower levels of depressive symptoms among participants $(\mathrm{B}=-.39, t=-9.15, p<.01)$, however, this relationship was rendered non-significant when our proposed mediators were entered in to the model, indicating that coping and stigma variables fully mediated the relationship between trait mindfulness and depressive symptoms in our community sample, $F(6,268)=71.35, p<$ .01. The model including all mediators accounted for a substantial $60.64 \%$ of the variance in depressive symptoms. As in the previous analysis, an examination of individual parameters indicated that the significance of this indirect effect was driven by internalized stigma and the endorsement of emotion-focused coping strategies. Specifically, trait mindfulness negatively predicted internalized stigma, which in turn 
negatively predicted depressive symptoms among participants $(\mathrm{CI}=-.21,-.11)$.

Similarly, emotion-focused coping $(\mathrm{CI}=-.31,-.18)$ significantly mediated the relationship between trait mindfulness and depressive symptoms, such that higher levels of mindfulness were associated with lower endorsement of emotion-focused coping strategies and fewer reported depressive symptoms. The confidence intervals for all other parameters, including problem-focused, avoidant and mindful coping all contained zero, indicating that these variables did not significantly mediate the relationship between trait mindfulness and depressive symptoms in the present sample (see Figure 2.2 for a summary of multiple mediation model). 


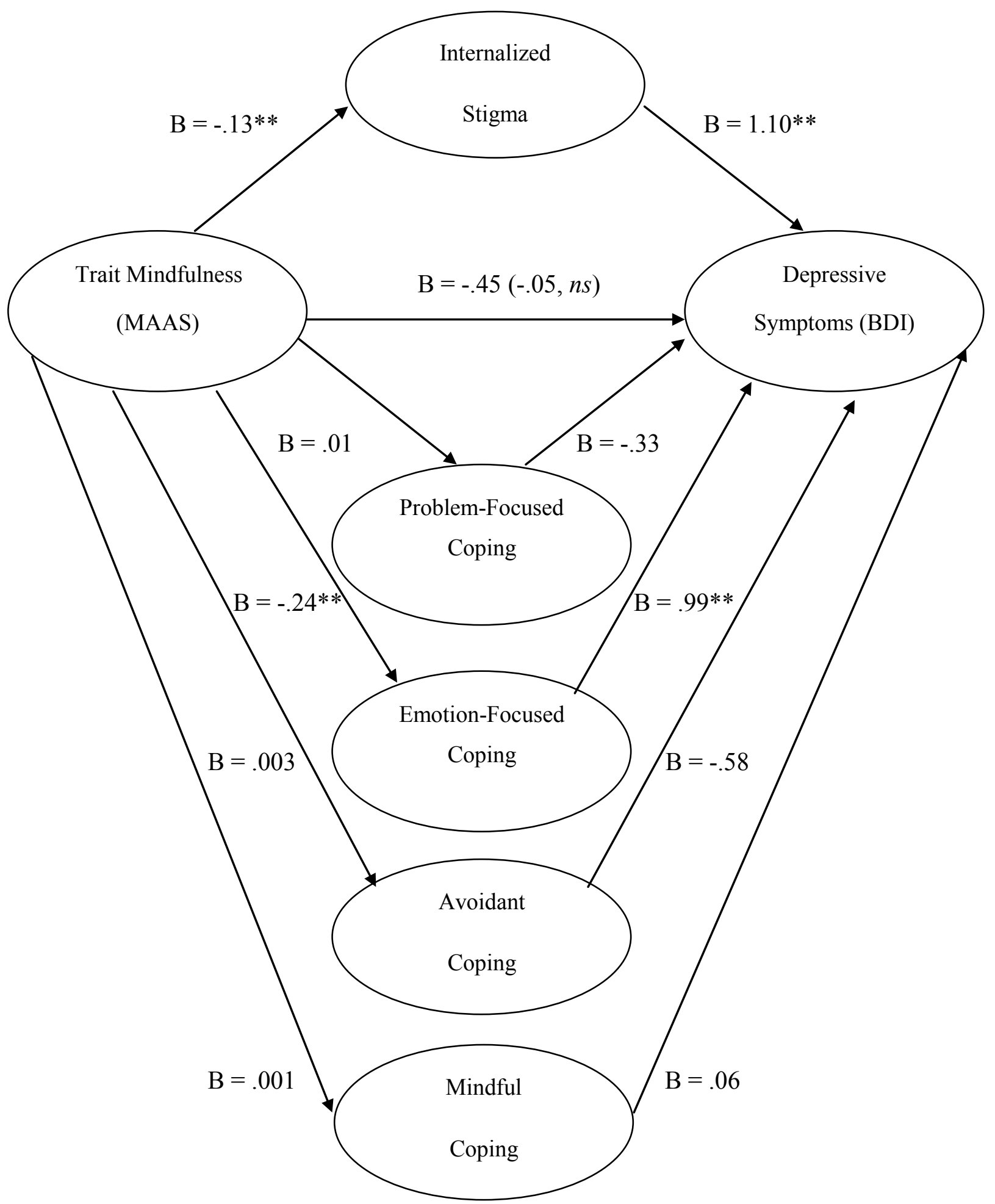

Figure 2.2. Internalized Stigma (ISMIS) and coping styles (problem-focused, emotionfocused, avoidant and mindful coping) as multiple mediators in the relationship between trait mindfulness and depressive symptoms in a community sample $\left({ }^{*} p<.05,{ }^{*} p<.01\right)$. 


\section{Discussion and Conclusions}

Previous literature has identified three primary forms of coping: problem-focused, emotion-focused and avoidant (Lazarus \& Folkman, 1984). Despite robust replication and validation of these three coping styles, however, it appears that individuals rarely adhere to a rigid coping repertoire. Instead, it has increasingly been argued that the successful navigation of stressors may hinge upon flexibility and the ability to draw upon different types of coping strategies in order to meet the unique demands and characteristics of situations as they unfold (Lazarus \& Folkman, 1986; Kato, 2012; Roussi, Krikeli, Hatzidimitriou \& Koutri, 2007). Furthermore, a growing number of researchers have acknowledged that multiple strategies, even those traditionally viewed as 'maladaptive', can be effective if used in an appropriate time and place (Lazarus \& Folkman, 1984). Although rumination is often described as a non-productive coping response when employed chronically or over long time horizons (e.g., Nolen-Hoeksema, 1991; 2000), for instance, some degree of rumination or reflective thinking following a stressful encounter might facilitate problem-solving, or promote a deeper understanding of one's reaction to a specific event, which in turn might inform a different and more effective coping response when a similar situation is encountered in the future (e.g., Watkins \& Moulds, 2005). Similarly, although wishful thinking about the potential outcome of a challenging or anticipated task might not be productive as a general, sustained coping strategy, such thinking might facilitate cognitive restructuring or problem solving efforts in the short-term (e.g., increased studying to achieve a desired grade on an exam). This research suggests that no single form of coping is consistently superior or inferior to another; rather, successful coping involves the ability to select and 
modify coping responses according to changing characteristics and demands of the situation at hand.

Increasing research in recent years has focused on mindfulness as a strategy for reducing stress and promoting well-being (e.g., Kabat-Zinn, 1990). Although trait mindfulness has been associated with autonomic recovery following stressful encounters and lower instances of depression (e.g., Segal, Williams \& Teasdale, 2002), little is known about the ways in which mindfulness produces these effects. As such, one purpose of the present investigation was to determine if mindful activities (e.g., focusing on present-moment responses) themselves serve as coping strategies for some individuals, to determine if these responses constitute a unique form of coping, and to determine if these mindful coping strategies were more commonly endorsed by young adults in response to specific types of stressors.

In line with previous research, some overlap was evident between emotionfocused, avoidant, problem-focused and mindful coping constructs in the present study, suggesting that these coping responses are not mutually exclusive. While most coping dimensions showed small to moderate correlations with one another, a strong, positive correlation was observed between the mindful coping and problem-solving subscales for both student and community samples, suggesting that individuals may endorse mindful coping techniques (e.g., focusing on the present moment, breathing, reminding oneself not to become preoccupied thinking about negative potential outcomes, etc.) in conjunction with problem-solving and/or strategies aimed at reducing the emotional impact of stressors. Despite the presence of some moderate to high correlations, 
however, confirmatory factor analysis indicated that these coping dimensions possess distinct factor structures, with several items loading strongly and uniquely on each factor.

Supporting our previous research, and consistent with prior literature (KabatZinn, 1990; Teasdale, Segal \& Williams, 1995), trait mindfulness negatively predicted depressive symptoms, internalized stigma, and appraisals of both threat and distress across student and community samples in the present study. As expected, trait mindfulness inversely predicted the endorsement of emotion-focused coping strategies across both samples, while mindful coping was negatively correlated with depressive symptoms, and positively correlated with control appraisals. Mindful coping was also negatively associated with appraisals of threat, although these relationships did not reach statistical significance.

Although mindful coping was expected to follow as a behavioural extension of trait mindfulness, this relationship was not supported in the present study; in fact, trait mindfulness failed to predict mindful coping efforts in either student or community samples. Interestingly, however, the endorsement of mindful coping strategies predicted a reliable decrease in distress appraisals and increases in perceptions of control over potentially stressful situations. In addition, individuals who endorsed the use of mindful coping strategies were more likely to evaluate the likely outcome of events favourably, relative to those who did not endorse such strategies.

There are several possible interpretations for the lack of an observed association between trait mindfulness and mindful coping in the present study. First, the trait mindfulness measure employed in this study assesses awareness of present-moment 
thoughts and sensations, but does not assess other theoretical aspects of mindfulness, including non-judgment and non-reacting to events (e.g., Baer, Smith, Hopkins, Krietemeyer \& Toney, 2006). The MAAS, therefore, may represent an inadequate or incomplete measure of trait mindfulness, one that fails to capture or differentiate between theorized aspects of mindfulness. While we initially expected that attention and awareness would facilitate subsequent coping efforts, it remains possible that presentmoment attention in itself is not sufficient to activate coping behaviour, and that mindful coping actions may stem from aspects of trait mindfulness that were not assessed in the present investigation (e.g., the ability to accept thoughts and feelings without reacting to them).

Alternatively, previous literature has suggested that mindfulness may help to attenuate negative cognitions (e.g., rumination) (Heeren \& Philippot, 2011) and physiological reactivity to stressors (Herwig et al., 2010; Teasdale, Segal, Williams, Ridgeway, Soulsby \& Lau, 2000). It follows that mindful individuals might be better equipped than their non-mindful counterparts to regulate emotion and to process and accept features of their environment, which in turn enables problem-solving and adaptive coping responses. In this way, trait mindfulness may act upon cognitions and physiological processes at a level that precedes the expression of external coping actions, but rather supports or informs the subsequent selection of coping responses.

In regards to the relationship between mindfulness and the use of coping strategies across domains, multiple regression analyses revealed that trait mindfulness consistently and negatively predicted the use of emotion-focused coping strategies (e.g., crying, rumination) in response to interpersonal and academic/workplace stressors. The 
only context in which this relationship was not uniformly observed was in the financial stressor domain, where gender differences in coping responses were apparent. Highly mindful women were less likely than non-mindful women to report the use of emotionfocused strategies in response to financial stressors, while men did not report the endorsement of any particular type of coping strategy, regardless of their level of trait mindfulness.

It is important to note that while individuals were asked to think of a recent and personally salient financial stressor, we did not qualify or control for the type or intensity of this stressor. It is possible, therefore, that some participants may have rated their use of coping strategies in response to relatively severe stressors (e.g., declaring bankruptcy), while others may have reported coping actions taken in response to comparatively milder stressors (e.g., losing \$10). Additionally, although a thorough discussion of gender differences is beyond the focus and scope of the present study, it should be noted that previous research has found gender differences in regards to vulnerability to financial stressors, with males tending to report both greater numbers of financial stressors, and greater distress as a result of these stressors (e.g., Roxburgh, 1997; Wheaton, 1990). This particular vulnerability has been hypothesized to stem from traditional gender roles and long-standing North American social norms that have heavily emphasized male responsibility in providing and maintaining financial security for their household. Given these observed trends, it is possible that this particular type of stressor was more salient to males than to females in the present study, or that men may have tended to recall more extreme, numerous, or distressing instances of financial instability or distress relative to women. If the types and characteristics of the financial situations rated by men were 
highly variable, they may have been approached or resolved through variable means as well, thus masking specific coping trends in this domain. A separate investigation would be needed to further examine potential gender differences in coping, while controlling for type and severity of financial stressors.

A final purpose of this study was to identify specific mechanisms through which trait mindfulness inversely predicts depressive symptoms. Mediation analyses supported the model identified in Study 1. Specifically, analyses of both student and community samples indicated that while trait mindfulness was correlated with diverse coping responses, the negative relationship observed between trait mindfulness and depressive symptoms was accounted for primarily through pathways involving stigma and emotionfocused coping. Higher trait mindfulness predicted decreases in both stigmatization for symptoms and endorsement of emotion-focused coping strategies in response to stressors, which in turn predicted lower depression scores. The consistent associations observed between trait mindfulness and suspension of emotion-focused coping strategies suggest that this pathway may represent an important mechanism through which mindfulness influences well-being and discourages reactivity to stressors and the development of depressive symptoms.

Despite these robust trends, the specific ways that trait mindfulness influences physiological reactivity during periods of acute stress remain largely unexplored, and it is not known if brief mindfulness training is effective in reducing reactivity and conferring meaningful improvements in coping and perceived anxiety following an acute stressor. To further investigate these relationships, the purpose of Study 3 was to examine the association between specific facets of trait mindfulness and stressor responses among 
young adults, and to further determine if brief mindfulness training encouraged more adaptive appraisals and physiological responses to stress, the use of mindful coping strategies, and resistance to internalized stigma and depression.

\section{STUDY 3.1}

Trait mindfulness has been associated with decreased heart rate and autonomic reactivity (Anderson, Mayberg, Bean, McKeon \& Segal, 2010; Zeidan, Johnson, Gordon \& Goolkasian, 2010), lower levels of circulating cortisol following a stressor challenge (Brown, Weinstein \& Creswell, 2012), and decreased neural activity in the dorsomedial prefrontal cortex and amygdala during the processing of emotional imagery (Frewen, Dozois, Neufeld, Lane, Densmore, Stevens, et al., 2010). Although this research suggests that trait mindfulness may help to attenuate physiological and emotional responses to stressors, little is known about the specific mechanisms that underlie these processes, and the ways in which mindfulness influences psychological, emotional and physiological responses to stressors.

The Mindful Attention and Awareness Scale (MAAS; Brown \& Ryan, 2006) has been cited as one of the most popular and commonly used empirical measures of trait mindfulness (Van Dam, Earleywine \& Borders, 2010). Given the high profile of this measure in published research, the MAAS has undoubtedly guided much of our scientific inquiry, operationalization of trait mindfulness, and understanding of this construct and its relation to well-being. The MAAS was designed to assess trait mindfulness as a unidimensional construct, focusing on present-moment attention and awareness (Brown \& Ryan, 2003). While this focus on present-moment attention is often viewed as being central to the concept of mindfulness (e.g., Brown \& Ryan, 2006; Kabat-Zinn, 1994), the 
treatment of this trait as a unidimensional construct has drawn criticism on the grounds that mindfulness may involve processes beyond attention and awareness. In a series of empirical studies (e.g., Baer, Smith, Hopkins, Krietemeyer \& Toney, 2006; Baer et al., 2008), five distinct facets of mindfulness have been identified, including "Acting with Awareness", "Observing Thoughts and Emotions", "Non-reacting to Inner Experience", "Describing and Labeling" (e.g., the ability to articulate thoughts and feelings) and "Nonjudging of Experience". Interestingly, preliminary research has indicated that these facets of mindfulness might differentially predict well-being and behaviour, including substance use among young adults (Eisenlorh-Moul, Walsh, Charnigo, Lynam \& Baer, 2012), depression and anxiety (Baer et al., 2008, Cash \& Whittingham, 2010), and neural suppression of automatic emotional responses (Paul, Stanton, Greeson, Smoski \& Wang, 2013). It may be clinically and empirically beneficial to adopt a facet approach in studies of mindfulness, as these facets may allow us to uncover more specific pathways of action and gain new insight into causal and predictive relationships between mindfulness, stress, coping and mental health. This specification, in turn, may inform the development of increasingly focused and customized clinical mindfulness interventions and programs (e.g., Cash \& Whittingham, 2010).

In light of this research, one goal of Study 3.1 was to determine if trait mindfulness was associated with lower resting/baseline cortisol and cytokine levels among individuals, and to determine if trait mindfulness predicted a diminished cortisol and cytokine response among young adults following an acute stressor task. A further goal of this study was to identify whether or not specific facets of mindfulness assessed by the FFMQ (“Acting with Awareness", "Observing”, "Non-reacting to Inner 
Experience", "Describing and Labeling" and "Non-judging") differentially predicted coping, well-being, and physiological responses to stressors. To replicate our previous investigations, we hypothesized that broad measures of trait mindfulness would inversely predict depressive symptoms among young adults. Since internalized stigma fundamentally occurs when individuals accept and internalize negative stereotypes and beliefs about mental illness (e.g., River \& Holmes, 1998; Rüsch, Corrigan, Todd \& Bodenhausen, 2010), and stigma may exacerbate depressive illness (e.g., Lundberg, Hansson, Wentz \& Bjorkman, 2009), we hypothesized that the facet of non-judgment would most strongly and uniquely predict reductions in internalized stigma and depressive symptoms among young adults (Baer et al., 2008, Cash \& Whittingham, 2010). Given findings by Paul et al. (2013), we further expected that facets of nonjudgment and non-reacting to experience would predict diminished cortisol and cytokine activity following a stressor task.

\section{Method}

\section{Participants}

Participants were recruited for this study through the SONA system at Carleton University (see Appendix I for recruitment notice). Exclusion criteria for this study included the presence of any medical conditions or medications (e.g., antiinflammatories) known to affect hormone function or release, extreme fear of needles and/or giving blood, and any previous history of nausea, fainting, or other complications as a result of giving blood. A total of 54 individuals completed an initial screening questionnaire (described below); of these, 30 met eligibility criteria and were invited to participate in the study. The remaining 24 students failed to meet eligibility criteria due 
to past experiences of nausea $(n=2,3.7 \%)$, dizziness $(n=4,7.4 \%)$, physical difficulties with blood donation including severe bruising and/or collapsed veins $(n=5,9.3 \%)$, severe anxiety regarding blood donation $(n=8,14.8 \%)$, having no prior experiences with needles or blood donation ( $n=2,3.7 \%)$, or current use of anti-inflammatory medications $(n=3,5.6 \%)$.

A total of 19 participants ( $n=3$ male; $n=16$ female) met all inclusion criteria for this study and provided complete blood samples for all time points. Participants ranged in age from $17-25$ years ( $M$ age $=20$ years, $S D=2.19$ years), and were predominantly of Caucasian $(n=8,42.1 \%)$ and Asian descent $(n=6,31.6 \%)$. All participants were enrolled in full-time studies at Carleton University. Four participants (21.1\%) reported that they had sought treatment for depression or anxiety in the past, although no participants reported current use of medications or engagement in treatment for these symptoms.

\section{Procedure}

All study procedures were approved by the Psychology Research Ethics Board and the Biohazards Research Review Committee at Carleton University. Prior to registering for the laboratory session, interested participants were directed to a brief online screening questionnaire to determine eligibility for this study (see Appendix I for recruitment notice). This questionnaire was hosted online through the SONA system at Carleton University. The purpose of this questionnaire was to identify any exclusion criteria, including the presence of medical conditions (e.g., diabetes, autoimmune disorders) or medications (e.g., anti-inflammatories) known to affect hormone function or release, extreme fear of needles and/or giving blood, and any previous history of nausea 
or fainting as a result of needles or giving blood. This questionnaire also asked participants about prior experiences with blood donation in order to determine, 1) if they had given blood before, 2) comfort levels with the blood sampling procedure, and 3) any physiological difficulties participants may have experienced with blood donation in the past (e.g., difficulty locating veins, extreme bruising/pain, collapsed veins, etc.). Participants were excluded from this study if they reported any discomfort with the blood collection procedure, adverse past reactions to donating blood, or the presence of any medical conditions or medications that would interfere with cortisol or cytokine production and release.

Participants meeting pre-screening criteria were invited to complete an in-lab study assessing individual differences in responding to challenging situations. Upon arriving at the lab, the researcher explained the laboratory session and verbally administered consent forms with participants to ensure full consent and compliance with the task and blood sampling procedures. Individuals were then given time to read and sign both a general informed consent form, as well as a separate consent form permitting the use of blood samples for analysis of cortisol and cytokines (see Appendix $\mathrm{J}$ for consent forms).

After obtaining consent, participants completed a short questionnaire package including both the Mindful Attention and Awareness Scale (MAAS; Brown \& Ryan, 2006) and the Five-Facet Mindfulness Questionnaire (Baer, Smith, Hopkins, Krietemeyer \& Toney, 2006), the Beck Depression Inventory (BDI; Beck, 1967) and the Internalized Stigma of Mental Illness Scale (ISMIS; Ritsher, Otilingam \& Grajales, 2003). Following completion of these questionnaires, the blood sampling procedure was started. A 
butterfly catheter was inserted into the participant's arm by a Registered Nurse, allowing blood samples to be drawn at various time points throughout the session, as noninvasively as possible. Once the catheter was inserted, the first blood sample was collected and participants were invited to relax for a 5-minute period. This waiting period was designed to give participants some time to acclimatize to the study location and blood withdrawal procedure, and to allow anxiety levels to diminish after insertion of the needle. The second blood sample was collected following this rest period. Neutralcontent reading material (a selection of home décor and Canadian history/Canadian Geographic magazines) were provided for participants to read during this acclimatization period.

Stressor task/social stressor paradigm. A modified version of the Trier Social Stress Test (TSST; Kirschbaum, Pirke, \& Hellhammer, 1993) was used to elicit psychosocial stress among participants. In this paradigm, participants were asked to deliver a speech about their employability to a panel of three graduate students for a period of 5 minutes (following a 5-minute preparation period). During this time, participants were not permitted to consult or read from notes made during the preparation phase, nor were they permitted to make any new notes to direct their speech. The researcher offered brief prompts (e.g., "Can you talk more about your past work experience?") only if participants did not speak for more than 30 seconds.

Following the speech task, participants completed an additional 5-minute mental arithmetic challenge, in which they were asked to subtract 17 from the number 1762. Responses were given aloud, and participants were asked to start again from the beginning if they made a mistake. To increase social-evaluative threat, graduate student 
"audience members" were instructed 1) not to speak with the participant at any time during the task, 2) not to offer physical signs of support or encouragement (e.g., smiling, nodding, or otherwise prompting a response), and 3) to maintain neutral facial expressions for the duration of the speech and mental math tasks. This task has been used extensively within laboratory settings, and has shown to serve as a reliable socioevaluative stressor across diverse samples (e.g. Kirschbaum, Pirke, \& Hellhammer, 1993; Kudielka, Helhammer \& Kirschbaum, 2007).

After the 5-minute preparation or waiting period, the third blood sample was collected, and participants were instructed to begin their assigned task (speech delivery or writing exercise). Participants then completed a final set of questionnaires, including the Positive and Negative Affect Schedule (PANAS; Watson, Clark \& Tellegen, 1988) and the State Anxiety Subscale of the State-Trait Anxiety Inventory (STAI; Spielberger, Gorsuch, Lushene, Vagg, \& Jacobs, 1983). Fifteen and 30 minutes after completing the task, the fourth and fifth blood sample was collected, after which the needle was removed from their arm. All participants were then debriefed and compensated for their time. Individuals received their choice of either $\$ 20 \mathrm{CAD}$, or $2 \%$ course credit if enrolled in an introductory psychology course for completing this laboratory session (see Appendix L for debriefing).

Blood sampling. A 19-gauge needle with a 5-foot line was used for blood withdrawal, and the rate of withdrawal over the 50-minute sampling period was monitored and controlled using a Dakmed continuous withdrawal ambulatory pump (Dakmed Inc., Buffalo, NY). The needle and line were covered with a surgical drape to prevent participants from viewing the blood withdrawal. Blood samples were collected 
continuously into chilled EDTA coated Vacutainer tubes. The volume of blood collected at each of the 5 primary sample points was approximately $2.75 \mathrm{~mL}$, with a total of approximately $35-40 \mathrm{~mL}$ of blood taken across the test period (including small interim amounts drawn between sampling times to prevent the line from clotting). Blood samples were immediately stored on ice for transport from the study location to the lab, and were centrifuged for 15 minutes at $4{ }^{\circ} \mathrm{C}$ and $2100 \mathrm{~g}$ within 30 minutes of collection. Plasma was then aliquoted into individual Eppendorf tubes and frozen at $-80^{\circ} \mathrm{C}$ until needed for cortisol and cytokine assays.

Cytokine and cortisol assay. Circulating levels of IL-6 and IL-10 were determined in duplicate by ELISA using high performance human IL-6 and IL-10 kits obtained from R \& D Systems (Minneapolis, MN). Cortisol was determined in duplicate by radio-immuno assay using kits obtained from ICN Biomedicals, CA. The assays were performed according to the manufacturer's instructions. The intra-assay variability was less than $10 \%$, and all samples were assayed in a single run to avoid inter-assay variability.

\section{Measures}

Prior to the laboratory session, participants completed a brief online screening questionnaire. Participants then completed an in-lab questionnaire package assessing depressive symptoms (BDI; Beck, 1967), internalized stigma (ISMIS; Ritsher, Otilingam \& Grajales, 2003), coping strategies (SCOPE; Matheson \& Anisman, 2003), and measures of post-task anxiety (STAI; Spielberger, Gorsuch, Lushene, Vagg, \& Jacobs, 1983) and affect (PANAS; Watson, Clark, \& Tellegen, 1988) (refer to Appendix K, and to Study 1 for a description of measures and scoring). In addition, participants completed 
measures assessing both unidimensional trait mindfulness (MAAS; Brown \& Ryan, 2006) and specific facets of trait mindfulness (FFMQ; Baer, Smith, Hopkins, Krietemeyer \& Toney, 2006). See Table 3.1.1 for a summary of reliability coefficients for all measures.

Pre-screening / eligibility questionnaire. All interested participants completed an initial pre-screening questionnaire to confirm eligibility for this study. Participants were asked to indicate ("Yes"/"No") whether or not they experienced any medical conditions (e.g., diabetes, autoimmune disorders) or currently used medications (e.g., anti-inflammatories, oral contraceptives) known to affect hormone function or release. Participants were also asked to indicate if they 1) had given blood before, and 2) had experienced any physiological difficulties that prevented or interfered with blood donation in the past (e.g., difficulty locating veins, extreme bruising/pain, collapsed veins, etc.). Finally, participants were asked to rate their anxiety level when thinking about needles and/or giving blood on a 10-point scale, ranging from 1 ('not anxious at all') to 10 ('extremely anxious'). Participants were excluded from this study if they reported any discomfort with the blood collection procedure, adverse past reactions to donating blood, extreme anxiety, or the presence of any medical conditions or medications that would interfere with cortisol or cytokine production and release.

Multi-dimensional trait mindfulness. The 39-item Five Facet Mindfulness Questionnaire (FFMQ; Baer, Smith, Hopkins, Krietemeyer \& Toney, 2006) was used to assess five distinct facets of mindfulness among participants; Acting with Awareness (e.g., "I find myself doing things without paying attention" [reverse scored]), Observing (e.g., "I pay attention to sounds, such as clocks ticking, birds chirping, or cars passing"), 
Non-reacting to Inner Experience (e.g., "I perceive my feelings and emotions without having to react to them"), Describing and Labeling (e.g., "I'm good at finding the words to describe my feelings"), and Non-Judging (e.g., "I think some of my emotions are bad or inappropriate and I shouldn't feel them" [reverse scored]). Items were rated on a 5point Likert scale, ranging from 1 ('Never/Very Rarely True') to 5 ('Very Often/Always True'). A total of 19 items were reverse coded as per conventional scoring guidelines (Baer et al., 2006). Responses were then summed and mean scores calculated for each of the five subscales, with higher scores representing greater mindfulness in the given domain/facet. This scale demonstrated high internal consistency reliability in the present sample (see Table 3.1.1 for a summary of internal consistency coefficients).

State anxiety. In order to assess perceived anxiety following the stressor task, participants completed the 20-item state anxiety subscale of the State-Trait Anxiety Inventory (STAI; Spielberger, Gorsuch, Lushene, Vagg, \& Jacobs, 1983). Statements (e.g., "I am tense", "I feel nervous") were rated on a 4-point Likert scale, ranging from 1 ('Not at all') to 4 ('Very much'). Half of the items (e.g., "I feel calm", "I feel comfortable") were reverse scored. A mean score was calculated for each participant, with higher scores representing greater state anxiety following completion of the laboratory task. Internal consistency reliability was high for the STAI in the present sample.

Affect. Participants completed a modified version of the Positive and Negative Affect Schedule (PANAS; Watson, Clark, \& Tellegen, 1988) to assess affect following the stressor task. Participants were asked to read a list of 10 adjectives representing positive affect (e.g., active, alert, attentive, strong, interested) and 10 adjectives 
representing negative affect (e.g., afraid, nervous, irritable, guilty, ashamed, distressed), and to rate the extent to which they felt each adjective in that moment, ranging from 0 ('Not at all') to 6 ('Extremely'). A mean score was calculated for both Positive Affect (PA) and Negative Affect (NA), with higher scores representing greater endorsement of Positive or Negative Affect, respectively. Internal consistency was high for both subscales in the present sample (see Table 3.1.1).

Table 3.1.1

Summary of Internal Consistency Coefficients for Measures Assessing Coping, Internalized Stigma, Trait Mindfulness and Depressive Symptoms $(N=19)$

\begin{tabular}{llc}
\hline \multicolumn{1}{c}{ Measure } & \multicolumn{1}{c}{ Subscale(s) } & Cronbach's $\boldsymbol{\alpha}$ \\
\hline Depressive Symptoms & -- & .80 \\
Internalized Stigma & -- & .80 \\
State Anxiety & -- & .95 \\
Trait Mindfulness (MAAS) & -- & .83 \\
Trait Mindfulness (FFMQ) & Acting with Awareness & .82 \\
& Observing & .81 \\
& Non-reacting to Experience & .77 \\
& Describing and Labelling & .94 \\
& Non-judging & .87 \\
& FFMQ total & .91 \\
Coping & Problem-focused & .83 \\
& Emotion-focused & .87 \\
& Avoidant & .62 \\
Affect & Mindful Coping & .65 \\
& Positive & .87 \\
& Negative & .94 \\
\hline
\end{tabular}




\section{Results}

\section{Preliminary Analyses}

Data were first screened for missing values and outliers. No participants had missing values on any items, and scores were within a plausible range for all measures. An examination of histograms and boxplots indicated that all variables were normally distributed and heteroscedastic. One case emerged as a potential univariate outlier on the FFMQ, but an examination of z-scores indicated that this case did not deviate substantially from the remaining cases $(z=-2.03)$; as such, this case was retained for further analyses. No multivariate outliers were apparent in the present sample.

Bivariate correlations were examined to identify the strength and direction of the relationship between unidimensional trait mindfulness (MAAS), specific facets of trait mindfulness (FFMQ), endorsement of various types of coping strategies, internalized stigma and depressive symptoms. As anticipated, the two mindfulness measures were highly correlated in the present sample, although not so highly correlated as to be considered redundant or overlapping measures (Tabachnick \& Fidell, 2007). As in previous investigations, scores on both measures of trait mindfulness were negatively associated with depressive symptoms, stigma, and the endorsement of emotion-focused coping strategies. Scores on the FFMQ were highly correlated with the use of mindful coping strategies, although this relationship was not observed between mindful coping and scores on the unidimensional MAAS. An examination of FFMQ subscales revealed moderate, positive correlations between mindfulness facets (ranging from $r=.22$ to $r=$ .58). Of particular interest, the facet of "non-judging of experience" was inversely and 
significantly associated with depressive symptoms among participants (see Table 3.1.2 for a summary of bivariate correlations). 
Table 3.1.2

Bivariate Correlations Between Unidimensional Trait Mindfulness (MAAS), Facets of Trait Mindfulness (FFMQ), Internalized Stigma, Depressive Symptoms, and Coping Strategies Endorsed $(N=19)$.

\begin{tabular}{|c|c|c|c|c|c|c|c|c|c|c|c|c|c|}
\hline & 1 & 2 & 3 & 4 & 5 & 6 & 7 & 8 & 9 & 10 & 11 & 12 & 13 \\
\hline 1. Trait Mindfulness (MAAS Total) & -- & & & & & & & & & & & & \\
\hline 2. Trait Mindfulness (FFMQ Total) & $.73 * *$ & -- & & & & & & & & & & & \\
\hline 3. Acting With Awareness & $.72 * *$ & $.83 * *$ & -- & & & & & & & & & & \\
\hline 4. Observing & $.60 * *$ & $.73^{* *}$ & .42 & -- & & & & & & & & & \\
\hline 5. Describing and Labelling & .39 & $.72 * *$ & $.55^{*}$ & $.49^{*}$ & -- & & & & & & & & \\
\hline 6. Non-judging & $.65^{* *}$ & $.69 * *$ & $.58 * *$ & .29 & .23 & -- & & & & & & & \\
\hline 7. Non-Reacting to Experience & .29 & $.67 * *$ & $.46^{*}$ & $.51^{*}$ & .22 & .40 & -- & & & & & & \\
\hline 8. Depressive Symptoms & -.28 & -.41 & -.25 & -.37 & -.09 & $-.53 *$ & -.26 & -- & & & & & \\
\hline 9. Internalized Stigma & -.20 & -.38 & -.09 & -.25 & -.20 & $-.52 *$ & -.29 & $.52 *$ & -- & & & & \\
\hline 10. Problem-Focused Coping & $.51^{*}$ & $.62 * *$ & $.48^{*}$ & .41 & .32 & $.56^{*}$ & $.53^{*}$ & -.41 & -.38 & -- & & & \\
\hline 11. Emotion-Focused Coping & $-.71 * *$ & $-.61 * *$ & $-.50^{*}$ & -.39 & -.16 & $-.79 * *$ & -.38 & .41 & .44 & -.43 & -- & & \\
\hline 12. Avoidant Coping & .01 & -.13 & -.20 & .01 & -.04 & -.07 & -.16 & .19 & .03 & .19 & .23 & -- & \\
\hline 13. Mindful Coping & .46 & $.66^{* *}$ & $.54 *$ & $.57^{*}$ & .36 & .40 & $.60^{* *}$ & -.39 & -.35 & $.82 * *$ & -.41 & .10 & -- \\
\hline
\end{tabular}
$* p<.05 ; * * p<.01$ 


\section{Main Analyses}

Extending upon previous investigations (e.g., Herwig, Kaffenberger, Lutz \& Brühl, 2010), we hypothesized that trait mindfulness would inversely predict depressive symptoms and internalized stigma. In particular, since negative self-schemas and selfcriticism have been strongly linked to depressive illness (e.g., Beck, 2008), we expected that non-judgment for thoughts and feelings would inversely predict depressive symptoms and the internalization of stigma for these symptoms.

Multiple linear regression indicated that although scores on the MAAS were negatively associated with depressive symptoms, this variable did not significantly predict depressive symptoms in the present sample, $F(1,17)=1.44, p=.25, n s, \mathrm{R}^{2}{ }_{\text {adj }}=$ .024. Interestingly, however, when individual facets of mindfulness were examined, nonjudging of experience emerged as a significant predictor of depressive symptoms among young adults, $\mathrm{B}=-3.01, S E=1.15, t=-2.61, p=.02$. As hypothesized, the direction of this effect indicated that greater non-judgment for personal thoughts and feelings predicted lower scores on the BDI.

Similarly, although scores on the MAAS were inversely associated with internalized stigma, this variable was not predictive of stigma among participants, $F(1$, $17)=.71, p=.41, n s, \mathrm{R}^{2}{ }_{\text {adj }}=.016$. Once again, however, an examination of FFMQ subscales indicated that non-judging of experience was a unique and significant predictor of internalized stigma among participants, $\mathrm{B}=-1.80, S E=.68, t=-2.65, p=.02$. As in the previous analysis and in line with our expectation, individuals who scored high in the facet of non-judgment reported significantly less self-stigmatization for depressive symptoms relative to those scoring low on this dimension of trait mindfulness. 
Trait mindfulness, coping, post-stressor anxiety and affect. A further goal of this study was to determine the extent to which facets of trait mindfulness predicted the use of specific coping strategies and reported anxiety and affect following an acute stressor task. Multivariate regression analyses were first performed to explore the relationship between trait mindfulness and participants' reported use of coping strategies during the speech and mental arithmetic task. This analysis revealed that overall scores on the MAAS did not significantly predict the use of coping strategies during the stressor task, $F(4,13)=1.66, p=.22$, partial $\eta^{2}=.338$, power $=.37$. However, regression analysis on the individual subscales of the FFMQ revealed that non-judgment of thoughts and feelings was a significant predictor of coping strategies used by participants during the stressor task, $F(4,10)=4.51, p=.02$, partial $\eta^{2}=.643$, power $=.78$. Follow-on analyses indicated that individuals who tended to suspend self-judgment for their thoughts and reactions reported using significantly fewer emotion-focused coping strategies during the stressor task, $\mathrm{B}=-3.34, S E=.93, t=-3.61, p<.01$, partial $\eta^{2}=.501$.

A second series of multivariate regression analyses were performed to examine unidimensional and multidimensional effects of trait mindfulness on post-task anxiety and positive and negative affect. In the first analysis, composite scores on the FFMQ significantly predicted psychological response following a stressor, $F(3,14)=4.44, p=$ .02 , power $=.76$, accounting for $48.7 \%$ of the variance in state anxiety and affect ratings. An examination of parameters indicated that scores on the FFMQ inversely predicted state anxiety, $\mathrm{B}=-.85, S E=.27, t=-3.12, p=.01$, partial $\eta^{2}=.378$, and marginally predicted reductions in negative affect following the social stressor task, $\mathrm{B}=-1.46, S E=$ $.76, t=-1.92, p=.07$, partial $\eta^{2}=.187$. Trait mindfulness scores on the FFMQ also 
predicted greater positive affect among participants post stressor task, $\mathrm{B}=1.59, S E=$ $.61, t=2.63, p=.02$, partial $\eta^{2}=.302$. Interestingly, scores on the unidimensional MAAS did not significantly predict state anxiety $(p=.96)$, positive affect $(p=.21)$ or negative affect $(p=.76)$ among participants in the present sample. Results of this analysis suggest that while mindful awareness in itself was not predictive of affective responses, higher endorsement of a range of mindfulness facets (e.g., non-judgment, acceptance, etc.) predicted lower anxiety and negative affect, and increased positive affect among participants following a social stressor task.

Mindfulness, cortisol and cytokine response. In light of foundational research linking mindfulness to both mental and physical health (e.g., Kabat-Zinn, 1990, Segal, Williams \& Teasdale, 2002), a growing body of research has sought to illuminate the relationship between mindfulness and physiological processes implicated in the stressor response. Preliminary research in this field has suggested that 8 -week mindfulness training programs might help to attenuate neurogenic inflammation (Rosenkranz, Davidson, MacCoon, Sheridan, Kalin \& Lutz, 2013) and slow autonomic activity in response to stressors (Zeidan, Johnson, Gordon \& Goolkasian, 2010), and that trait mindfulness might predict diminished cortisol release following an acute stressor task (Brown, Weinstein \& Creswell, 2012). Although these studies suggest that mindfulness might help to attenuate harmful effects of stress, research on mindfulness and neuroendocrine response is still in its infancy, and further research is needed to clarify the contribution of mindfulness to subsequent physiological processes. Furthermore, published studies in this area to date have employed varying methods to assess both mindfulness and stressor response, making it difficult to compare reported effects and 
conclusions. In this regard, further research is needed to support and synthesize findings from different neurobiological perspectives, and to allow for a more comprehensive understanding of the ways and conditions under which mindfulness influences responses to stressful stimuli.

To build upon this research, a primary goal of the present study was to determine whether specific facets of trait mindfulness predicted lower baseline cortisol levels and a diminished plasma cortisol and cytokine response following an acute stressor task. Regression analyses revealed that the facet of observing thoughts and feelings significantly predicted baseline cortisol among participants, $\mathrm{B}=-6.62, S E=2.71, t=$ $2.45, p=.03$. The direction of this effect indicated that the tendency to observe and attend to thoughts and feelings predicted lower baseline levels of cortisol among participants.

A series of multiple regression analyses were then performed to determine if specific facets of trait mindfulness predicted neuroendocrine response following an acute stressor task. Subscales of the FFMQ were entered in to the model as continuous predictors, and change scores for cortisol, TNF- $\alpha$, IL-6 and IL-10 levels were calculated (from baseline to post-stressor task), serving as outcome variables, respectively. Although the five facets of trait mindfulness failed to predict cortisol response across the task session when modeled simultaneously, $F(5,12)=1.72, p=.21$, the power to detect statistical significance was low in this analysis (power $=.41)$. Follow-on analyses of individual facets suggested that the facet of observing thoughts and feelings was uniquely associated with cortisol response from baseline to post-task, $F(1,17)=4.31, p=.05$, partial $\eta^{2}=.155$, such that the tendency to observe and attend to thoughts and feelings 
predicted a diminished cortisol response among participants, $\mathrm{B}=-30.02, S E=14.46, t=$ $-2.08, p=.05$.

Subsequent multiple regression analyses indicated that facets of trait mindfulness did not significantly predict changes in TNF- $\alpha, F(5,11)=.11, p=.99$, and was not significantly associated with changes in IL- $6, F(5,11)=.27, p=.92$, or IL-10 in the present study, $F(5,11)=.72, p=.62$.

\section{Discussion and Conclusions}

It had been suggested that trait mindfulness is associated with lower rates of depression, suppression of negative emotional reactions, and the attenuation of stressor responses (Brown, Weinstein \& Creswell, 2012). Extending upon this research, the purpose of the present investigation was to examine the relationship between specific theorized facets of trait mindfulness and 1) depressive symptoms, 2) internalized stigma for depressive symptoms, 3) endorsement of coping strategies, 4) psychological stressor responses (e.g., perceived anxiety and affect), and 5) physiological stressor responses (e.g., changes of cortisol and cytokines) following an acute stressor.

As in our previous investigations, and consistent with previous literature (Lysaker, Roe \& Yanos, 2007; Moses, 2009; Rüsch, Corrigan, Todd \& Bodenhausen, 2010), internalized stigma was highly predictive of depressive symptoms in the present study. An examination of trait mindfulness facets revealed that non-judgment for thoughts and feelings was significantly and inversely associated with depressive symptoms, internalized stigma, and the endorsement of emotion-focused coping strategies when engaging in a stressful task. As hypothesized, individuals who tended to experience their thoughts and emotions without judgment reported internalizing negative 
stereotypes to a lesser extent than those who tended to evaluate their experiences more judgmentally, and reported fewer depressive symptoms relative to their more judgmental counterparts. Furthermore, higher trait mindfulness scores on the FFMQ predicted lower anxiety and negative affect following the stressor task, and an increase in positive affect ratings. Thus, it seems that trait mindfulness may be associated with less frequent use of emotionally-based coping techniques, and lower levels of negative emotion and anxiety within the context of acutely stressful situations.

With respect to the relationship between trait mindfulness and neuroendocrine response, the facet of mindful observing (i.e., noticing and attending to thoughts and feelings) significantly predicted baseline cortisol among participants, such that the tendency to observe and attend to thoughts and feelings predicted lower baseline levels of cortisol among participants. This facet also emerged as a significant predictor of cortisol response across a stressful task. Specifically, individuals who tended to observe and attend to their thoughts and feelings demonstrated lower cortisol levels when performing a stressful task, relative to individuals who did not endorse this skill. This result suggests that mindful observation of experiences may be associated with a diminished cortisol response during acutely stressful tasks.

Despite somewhat low statistical power in the present analyses, non-judgment of thoughts and feelings significantly predicted lower stigma and depression scores across participants. This finding is encouraging, suggesting that this aspect of trait mindfulness may be particularly useful in predicting those at risk for developing symptoms and internalizing negative stereotypes regarding depressive illness. Extending upon this, mindfulness-based programs that emphasize non-judgment of thoughts and feelings 
might be especially effective in discouraging self-stigma among individuals at risk of depressive illness or relapse. Replication of this study with a larger sample would afford greater statistical power to detect effects, and would further support the observed relationships between facets of mindfulness and well-being (e.g., Baer, Smith, Hopkins, Krietemeyer \& Toney, 2006). In addition, the inclusion of a control (e.g., non-stressor) group to the present design would allow for more complex comparisons, and may help to clarify the contribution of trait mindfulness to neuroendocrine response following an acute stressor task.

\section{STUDY 3.2}

In light of the negative associations between mindfulness, internalized stigma and depressive symptoms observed in the previous studies, it appears that mindfulness may help to reduce negative appraisals and protect individuals from internalizing negative self-information and stigma regarding depressive symptoms. As these studies were correlational in nature, however, the directionality of these effects is not known, and cause-and-effect relationships are difficult to ascertain. In addition, although mindfulness training (e.g., MBSR) has been purported to diminish negative mood and cardiovascular reactivity (Astin \& Vieten, 2008; Farb, Anderson, Mayberg, Bean, McKeon \& Segal, 2010; Zeidan, Johnson, Gordon \& Goolkasian, 2010), the extent to which mindfulness promotes well-being by attenuating cortisol and cytokine responses to acute stressors is unknown. Study 3.2 was designed as a laboratory study in order to investigate these relationships more fully, and to identify possible causal relationships between these variables. Specifically, the goals of Study 3.2 were to 1) replicate and support the relationships observed in our previous investigation between facets of mindfulness, 
depressive symptoms, coping and stigma, 2) determine if brief mindfulness training predicts the use of more active, mindful coping strategies in response to stressors, and 3) assess the relationship between mindfulness, subjective stress indices (e.g., perceived anxiety, positive and negative affect), and physiological responses to a stress paradigm (i.e., changes in cortisol and cytokine reactivity).

\section{Method}

\section{Participants}

A total of 121 male and female participants registered for this study through the SONA system at Carleton University (see Appendix M for recruitment notice). As in the previous investigation, participants were excluded from this study if they indicated the presence of any medical conditions or medications (e.g., anti-inflammatories, autoimmune disorders) known to affect hormone function or release, extreme anxiety or fear of needles and/or giving blood, or any previous history of nausea, fainting, collapsed veins, or other complications as a result of needles or giving blood. A total of 87 participants ( $n=34$ male; $n=53$ female) met all inclusion criteria for this study and completed both sessions. Participants ranged from 16 to 51 years of age $(M$ age $=20.48$ years, $S D=4.87$ years), and were predominantly Caucasian $(n=48,55.2 \%)$, with remaining participants reporting East Asian $(n=10,11.5 \%)$, Middle Eastern $(n=7$, 8.0\%), South Asian ( $n=6,6.9 \%)$, African Canadian/American $(n=2,2.3 \%)$, or 'Other' ethnicity $(n=7,8.0 \%)$. The vast majority of students were registered in full-time studies ( $n=82,94.3 \%)$, two participants were part-time students (2.3\%), and the remaining 3 participants were registered with special/undeclared student status (3.4\%). 


\section{Procedure}

Individuals who agreed to participate in this study were invited to complete a series of two in-lab sessions, scheduled approximately one week apart. These sessions were held on campus at Carleton University. As in the previous investigation, all study procedures were approved by the Psychology Research Ethics Board and Biohazards Research Review Committee at Carleton University.

Session 1. Participants were invited to an initial group session, comprising $6-12$ participants. Upon arriving at the lab, participants were asked to read and sign an informed consent form (see Appendix N) and to complete a brief demographic and medical questionnaire to confirm their eligibility for this study. Participants were then randomly assigned to a mindfulness, problem-solving, or active control condition.

Mindfulness Condition. In the mindfulness condition, individuals were informed that mindfulness is a personal characteristic that might facilitate well-being and problem solving. Participants were then advised that they would be learning a number of mindfulness-related exercises, designed to develop their awareness of moment-tomoment events. Participants were guided through a specific series of exercises, described by Segal, Williams and Teasdale (2002) and used as a part of the Mindfulness-Based Cognitive Therapy (MBCT) program as a means of developing greater awareness, mindful attention, and non-automaticity ${ }^{5}$ (see Appendix O for Mindfulness training scripts).

\footnotetext{
5 Although not certified MBCT instructors, the researchers leading this training session completed a professional MBCT training course with Drs. Zindel Segal and Susan Woods, and had previous practical experience and supervision in administering these exercises.
} 
Raisin Exercise. To begin, the experimenter placed a raisin in each participant's palm. The experimenter then instructed individuals to observe the raisin as though they have never seen the object before, and guided participants through a series of instructions for slowly and deliberately seeing, touching, and tasting the raisin. This exercise is designed to break automatic thinking, and to encourage greater awareness of everyday objects and activities.

Mindful Meditation. Participants were asked to sit in a comfortable position, and were guided through a 15-minute Mindful Meditation procedure, as described by Segal, Williams and Teasdale (2002). The purpose of this meditation was to draw participants' attention towards internal processes (the movement of breath, awareness of any physical/bodily sensations), and to acknowledge these responses "in the moment" as they arise. The goal of this activity is to develop a greater awareness of these sensations, to experience them without trying to change or control them, and to practice setting aside any thoughts or emotions that might intrude or automatically accompany these sensations (i.e., ruminating on an injury that may have contributed to sensations of pain, etc.).

3-Minute Breathing Space. Again, participants were asked to sit in a comfortable position and close their eyes. The researcher then guided individuals through a 3-minute breathing space exercise. The purpose of this exercise was to first focus awareness on any thoughts that are present (minute 1), to then slowly release these thoughts and move attention to the breath (minute 2), and finally to extend awareness to the whole body sitting in the present place and time (minute 3) (Segal, Williams \& Teasdale, 2002). The purpose of this exercise is to first notice the contents of one's 
thoughts, without judging or attempting to control or change these thoughts, and to then systematically re-focus attention on the breath and body in the present moment.

Once these exercises were completed, participants were provided with a list of handouts (see Appendix R) explaining the relationship between these exercises and the development of mindfulness. Participants were also provided with a copy of the Pleasant Events calendar (see Appendix S), and were asked to complete this calendar as a "homework" exercise each evening leading up to the second study session. This exercise asked individuals to reflect upon a positive and negative event each day, to note these events as they occur, and to record the physical, emotional and cognitive responses they experience concurrently with these events.

Analytic Problem-Solving Condition. In the analytic problem-solving condition, participants were informed that analytical skill is a personal characteristic that might facilitate well-being and problem solving ability. Participants were then asked to complete a number of puzzles requiring analytic thinking (Sudoku, crossword and logicbased word puzzles) for approximately 30 minutes (see Appendix P for problem-solving script and materials). In order to keep participants engaged with this activity, and to better match the tone and task requirements of the mindfulness condition, participants were asked to write a list of strategies they used to solve each type of puzzle. For the second half of the session (approximately 15 minutes), participants were asked to form a list of what they believed to be the most effective problem-solving strategies. To match the task demands of the mindfulness condition, participants were provided with an Analytic Thoughts calendar (modified from the Pleasant Events calendar, see Appendix S), which they were asked to complete as a homework exercise each evening leading up 
to the second study session. This exercise asked individuals to reflect upon a problem or event they experienced each day, to note what the event was, and to note any analytic or problem-solving steps they took to resolve the event (i.e., thought about the event, broke down a problem into smaller parts, etc.).

Active Control (Non-Training) Condition. In the control (i.e., non-training) condition, participants were asked to choose an activity that they enjoy and that they feel describes them as a person (e.g., participation in sports, music, volunteering, etc.). They were then asked to write about this activity, using a series of guiding questions (e.g., How long have you been participating in this activity? How do you feel about yourself when you engage in this activity?). See Appendix Q for control condition script and materials.

Following the assigned training or control tasks, participants completed an additional questionnaire assessing their endorsement of various coping strategies (SCOPE; Matheson \& Anisman, 2003). Session 1 took approximately 1.5 hours to complete. At the end of this session, participants were debriefed and scheduled a time with the researchers to complete the second portion of the study (see Appendix $\mathrm{T}$ for Session 1 debriefing). The second session was scheduled to take place in the afternoon (to control for diurnal changes in cortisol and circulating hormones) approximately 1 week after the initial training session. Participants were compensated $\$ 10 \mathrm{CAD}$ or $1.5 \%$ course credit for completing this portion of the study.

Session 2. Upon arriving at the lab, participants were reminded of the purpose and length of the second session, and had the opportunity to ask any questions they had regarding the previous session. The researcher then explained the details of the 
laboratory session and went through the consent forms verbally with all participants to ensure understanding and full consent and compliance with the task and blood sampling procedures. Individuals were then given time to read and sign a general informed consent form, as well as a separate consent form permitting the use of blood samples for analysis of cortisol and cytokine reactivity (see Appendix U for consent forms).

After obtaining consent, participants were asked to submit their homework assignments for the week (Positive and Negative events calendar or Analytic Thoughts calendar), and to complete a brief questionnaire rating the first session, including how informative, interesting and useful they found the session to be, as well as how effectively they were able to integrate the skills they learned in their daily lives over the one week period since the session (see Appendix V). At this time, participants also completed the Beck Depression Inventory (BDI; Beck, 1967) and the Internalized Stigma of Mental Illness Scale (ISMIS; Ritsher, Otilingam \& Grajales, 2003). Following completion of these questionnaires, the blood sampling procedure was started. A butterfly catheter was inserted into the participant's arm by a Registered Nurse, allowing blood samples to be drawn at various time points throughout the session, as noninvasively as possible. Once the catheter was inserted, the first blood sample was collected and participants were invited to relax and look through neutral-content magazines (home décor, etc.) for a 5-minute period. This waiting period was designed to give participants some time to acclimatize to the study location and blood withdrawal procedure, and to allow anxiety and hormone levels to stabilize after insertion of the needle. The second blood sample was collected following this rest period. 
Participants were then randomly assigned to either a stressor or control condition. Assignment to these groups was counterbalanced, such that an equal number of participants in the mindfulness, problem-solving and control conditions were allocated to each of the stressor and non-stressor conditions.

Stressor condition. As in the previous study, participants in the stressor condition completed a modified version of the Trier Social Stress Test (TSST; Kirschbaum, Pirke, \& Hellhammer, 1993), in which they were asked to deliver a short speech about their employability to a panel of three graduate students, followed by a short mental arithmetic task. They were given 5 minutes to prepare their speech.

Control condition. In the control condition, participants were told that an increasing number of students are now working outside of school in order to supplement their income or cover costs related to education. They were advised that the researchers were interested in learning more about the frequency of student employment outside of school, as well as gaining insight into the types of jobs that are most popular and attractive to students. Rather than presenting a speech to a panel of graduate students, individuals in this condition were asked to provide written responses to a short series of employment-related questions (e.g., whether or not they are currently employed outside of school, what jobs they think are most popular for students today, and what types of student jobs they believe are likely to become more popular over the next 10 years). They were then given 5 minutes to relax and look through neutral content magazines prior to beginning the writing exercise. 
After the 5-minute preparation or waiting period, the third blood sample was collected, and participants were instructed to begin their assigned task (speech delivery or writing exercise). Participants then completed a final set of questionnaires, including the Positive and Negative Affect Schedule (PANAS; Watson, Clark \& Tellegen, 1988) and the State Anxiety subscale of the State-Trait Anxiety Inventory (STAI; Spielberger, Gorsuch, Lushene, Vagg, \& Jacobs, 1983). Fifteen minutes after completing the task, the fourth blood sample was collected and the butterfly catheter removed. All participants were then debriefed and compensated for their time. Individuals received their choice of either $\$ 20 \mathrm{CAD}$, or $1.5 \%$ course credit if enrolled in an introductory psychology course for completing this session (see Appendix W for debriefing).

Blood sampling. As in the previous study, blood was withdrawn continuously using a 19-gauge needle with a 5-foot line, and the rate of withdrawal over the 40-minute sampling period was controlled using a Dakmed continuous withdrawal pump (Dakmed Inc., Buffalo, NY). The needle and line were covered with a surgical drape to prevent participants from viewing the blood withdrawal, and blood samples were collected into chilled EDTA coated Vacutainer tubes and immediately stored on ice for transport to the lab. The volume of blood collected at each primary sample point was approximately $2.75 \mathrm{~mL}$, with a total of $30 \mathrm{~mL}$ of blood taken across the test period (including small interim amounts drawn between sampling times to prevent the line from clotting). Blood samples were centrifuged for 15 minutes at $4^{\circ} \mathrm{C}$ and $2100 \mathrm{~g}$, and plasma was aliquoted into individual Eppendorf tubes. All samples were centrifuged within 30 minutes of collection and frozen at $-80^{\circ} \mathrm{C}$ until cortisol and cytokine assays were processed. 
Cytokine and cortisol assay. Circulating levels of IL-6 and IL-10 were determined in duplicate by ELISA using high performance human IL-6 and IL-10 kits obtained from R \& D Systems (Minneapolis, MN). Cortisol was determined by radioimmuno assay using kits obtained from ICN Biomedicals, CA. The assays were performed according to the manufacturer's instructions. The intra-assay variability was less than $10 \%$, and assays were analyzed in a single run to eliminate inter-assay variability.

\section{Measures}

As in the previous Study, participants completed a battery of questionnaires assessing trait mindfulness (MAAS and FFMQ), depressive symptoms, and internalized stigma prior to the laboratory task (see Study 1 for a description of measures and scoring). Participants were also asked to complete brief questionnaires to provide background information and feedback on the initial training session. In order to assess positive and negative affect and reported anxiety following the stressor or control task, participants were additionally asked to complete measures assessing state anxiety and mood/affect following completion of the assigned task (see Appendices $\mathrm{K}$ and $\mathrm{V}$ for Study 3 measures). A summary of internal consistency coefficients is presented in Table 3.2.1.

Background information. Participants were asked to complete a brief background questionnaire, assessing the use of any current medications (e.g., antiinflammatories), medical conditions (e.g., auto-immune disorders) or treatments that might influence cortisol and cytokine levels. 
Session 1 feedback. In order to assess the efficacy of Session 1, participants were asked to rate the previous session (mindfulness training, analytical problem-solving training, or control/no training condition). Participants were asked to rate how interesting, informative and useful they found the session to be on a 7-point Likert scale, ranging from 1 ('Not at all') to 7 ('Extremely'). An open-ended question was also included to allow participants to provide any additional feedback, comments, concerns, or suggestions for improvement on Session 1.

Social stressor paradigm. As in the previous study, participants assigned to the stressor condition completed a modified version of the Trier Social Stress Test (Kirschbaum, Pirke, \& Hellhammer, 1993), in which they delivered a 5-minute speech to a panel of graduate students, followed by a 5-minute mental arithmetic task. After completing this task, participants were invited to continue with a final set of questionnaires assessing state anxiety, positive and negative affect, and coping strategies employed during the stressor task (see Study 3.1 for a complete description of the social stressor task).

State anxiety. In order to assess perceived anxiety following the stressor or control task, participants completed the 20 -item state anxiety subscale of the State-Trait Anxiety Inventory (STAI; Spielberger, Gorsuch, Lushene, Vagg, \& Jacobs, 1983). Statements (e.g., "I am tense", "I feel nervous") were rated on a 4-point Likert scale, ranging from 1 ( 'Not at all') to 4 ('Very much'). Half of the items (e.g., "I feel calm", "I feel comfortable") were reverse scored. A mean score was calculated for each participant, with higher scores representing greater state anxiety following completion of 
the assigned stressor or control task. Internal consistency reliability was high for the STAI in the present sample (Cronbach's $\alpha=.93$ ).

Affect. Participants completed a modified version of the Positive and Negative Affect Schedule (PANAS; Watson, Clark, \& Tellegen, 1988) to assess affect following the stressor or control task. Participants were asked to read a list of 10 adjectives representing positive affect (e.g., active, alert, attentive, strong, interested) and 10 adjectives representing negative affect (e.g., afraid, nervous, irritable, guilty, ashamed, distressed), and to rate the extent to which they felt each adjective in that moment, ranging from 0 ('Not at all') to 6 ('Extremely'). A mean score was calculated for both Positive Affect (PA) and Negative Affect (NA), with higher scores representing greater endorsement of Positive or Negative Affect, respectively. Internal consistency was high for both subscales in the present sample (see Table 3.2.1). 
Table 3.2.1

Summary of Internal Consistency Coefficients for Measures Assessing Coping, Internalized Stigma, Trait Mindfulness and Depressive Symptoms $(N=87)$

\begin{tabular}{llc}
\hline \multicolumn{1}{c}{ Measure } & \multicolumn{1}{c}{ Subscale(s) } & Cronbach's $\boldsymbol{\alpha}$ \\
\hline Depressive Symptoms & -- & .87 \\
Internalized Stigma & -- & .87 \\
State Anxiety & -- & .93 \\
Trait Mindfulness (MAAS) & -- & .78 \\
Trait Mindfulness (FFMQ) & Acting with Awareness & .84 \\
& Observing & .75 \\
& Non-reacting to Experience & .79 \\
& Describing and Labelling & .92 \\
& Non-judging & .92 \\
& FFMQ total & .89 \\
Coping & Problem-focused & .84 \\
& Emotion-focused & .85 \\
& Avoidant & .72 \\
Affect & Mindful Coping & .71 \\
& Positive & .87 \\
& Negative & .92 \\
\hline
\end{tabular}

\section{Results}

\section{Preliminary Analysis}

Prior to conducting main analyses, data were screened for accuracy and missing values, and basic assumptions for multivariate ANOVA (including linearity, normality and outlying cases) were tested at each level of training and task condition. Homogeneity 
of variance was met in the present sample, and sample sizes were equal for each group ${ }^{6}$. Independence of observations was assumed given the nature of the study design (e.g., measures were completed individually, and participants' responses were not expected to influence the responses of others). All observed scores were within range for these variables, and no participants had missing values on the measures of interest. Boxplots and z-scores were examined, and no univariate outliers were apparent at any level of the independent variable (no z-scores exceeded 2.82). Similarly, an examination of Mahalanobis distance revealed no multivariate outliers across any level of the independent variables.

\section{Analysis of completer vs. non-completer samples. A series of one-way} ANOVAs were conducted to determine if participants who withdrew from the study after the first session differed systematically from participants who completed both study sessions on measures of trait mindfulness, internalized stigma or depressive symptomatology. These analyses revealed that the non-completing sample $(M=41.50$, $S D=7.43)$ did not differ from the completing sample $(M=44.33, S D=7.73)$ on composite scores on the MAAS, $F(2,118)=1.22, p=.30$; nor did non-completers $(M=$ $3.06, S D=.42)$ differ from study completers $(M=3.24, S D=.48)$ on trait mindfulness scores as assessed by the FFMQ, $F(2,118)=.86, p=.43$. Similarly, the two groups did not differ on depressive symptoms (completer $M=7.39$, $S D=6.37$; non-completer $M=$ $7.80, S D=6.12), F(2,118)=.07, p=.80$ or measures of internalized stigma (completer $M=7.52, S D=2.57$; non-completer $M=7.25, S D=3.32), F(2,118)=.17, p=.69$,

\footnotetext{
6 The ratio of largest group to smallest group in the present sample was 17 (assignment to mindfulness/stressor condition)/12 (assignment to control/control condition), giving a ratio of 1.42. This value falls below the ratio of 1.5 recommended by Tabachnick and Fidell (2006) to ensure equal sample sizes.
} 
indicating that attrition of participants was not explained by pre-existing differences in depressive symptomatology, stigma, or trait mindfulness.

Additional chi-square tests were then performed to identify any differences between completer and non-completer samples as a function of categorical demographic variables. These analyses indicated that completers and non-completers did not differ in terms of gender, $\chi^{2}(2)=1.74, p=.42$, ethnicity, $\chi^{2}(14)=7.05, p=.93$, or Session 1 condition (e.g., assignment to mindfulness, problem-solving or control group), $\chi^{2}(4)=$ $7.67, p=.10$. Based on these analyses, it appeared that attrition of participants from session 1 to session 2 was random, and was not attributable to systematic differences on any key variables under investigation.

Bivariate correlations. Bivariate correlations were examined to identify the strength and direction of the relationship between trait mindfulness, coping, internalized stigma, depressive symptoms, and reported affect and anxiety. As expected, both measures of trait mindfulness were inversely associated with depressive symptoms, internalized stigma, and the endorsement of emotion-focused coping strategies. Depressive symptoms were positively associated with stigma and negative affect following the stressor task, while non-judgment of thoughts and feelings was inversely correlated with post-task anxiety scores. See Table 3.2.2 for a summary of bivariate correlations. 
Table 3.2.2

Bivariate Correlations Between Trait Mindfulness, Internalized Stigma, Depressive Symptoms, Anxiety, Affect, and Coping Strategies

Endorsed $(N=87)$

\begin{tabular}{|c|c|c|c|c|c|c|c|c|c|c|c|c|c|c|c|c|}
\hline & 1 & 2 & 3 & 4 & 5 & 6 & 7 & 8 & 9 & 10 & 11 & 12 & 13 & 14 & 15 & 16 \\
\hline 1. Mindfulness-MAAS & -- & & & & & & & & & & & & & & & \\
\hline 2. Mindfulness-FFMQ & $.47 * *$ & -- & & & & & & & & & & & & & & \\
\hline 3. Awareness & $.64 * *$ & $.72 * *$ & -- & & & & & & & & & & & & & \\
\hline 4. Observing & .05 & $.28 * *$ & .09 & -- & & & & & & & & & & & & \\
\hline 5. Describing/Labelling & $-.25 *$ & $.77 * *$ & $.45^{* *}$ & .08 & -- & & & & & & & & & & & \\
\hline 6. Non-Judging & $.34 * *$ & $.70 * *$ & $.46^{* *}$ & $-.21 *$ & $.37 * *$ & -- & & & & & & & & & & \\
\hline 7. Non-Reacting & .15 & $.62 * *$ & $.21 *$ & .16 & $.40 * *$ & $.30 * *$ & -- & & & & & & & & & \\
\hline 8. Depressive Symptoms & $-.34 * *$ & $-.44 * *$ & $-.25 *$ & -.11 & $-.35 * *$ & $-.31 * *$ & $-.32 * *$ & -- & & & & & & & & \\
\hline 9. Internalized Stigma & $-.41 * *$ & $-.60 * *$ & $-.46^{* *}$ & -.01 & $-.44 * *$ & $-.52 * *$ & $-.36 * *$ & $.53 * *$ & -- & & & & & & & \\
\hline 10. Problem-Focused & .03 & .12 & -.01 & .15 & $.25^{*}$ & .03 & -.12 & $-.21 *$ & .05 & -- & & & & & & \\
\hline 11. Emotion-Focused & $-.28 * *$ & $-.48 * *$ & $-.30 * *$ & .01 & $-.35 * *$ & $-.46^{* *}$ & $-.33 * *$ & $.44 * *$ & $.57 * *$ & .15 & -- & & & & & \\
\hline 12. Avoidant Coping & .01 & .19 & -.03 & .09 & .13 & .19 & .18 & $-.26^{*}$ & $-.23 *$ & .04 & .04 & -- & & & & \\
\hline 13. Mindful Coping & -.01 & $.24 *$ & -.05 & $.26^{*}$ & .14 & .17 & $.27^{*}$ & $-.25 *$ & -.20 & $.28 * *$ & -.18 & $.31 * *$ & -- & & & \\
\hline 14. State Anxiety & -.20 & $-.24 *$ & -.20 & .01 & -.16 & $-.23 *$ & -.15 & $.42 * *$ & $.23 *$ & -.07 & $.32 * *$ & -.12 & -.16 & -- & & \\
\hline 15. Positive Affect & .16 & .14 & .19 & -.06 & .16 & .10 & -.02 & $-.23 *$ & $-.25^{*}$ & -.01 & -.13 & -.02 & -.03 & $-.31 * *$ & -- & \\
\hline 16. Negative Affect & -.05 & -.11 & -.03 & .03 & -.04 & -.15 & -.14 & $.32 * *$ & .14 & .01 & $.23 *$ & -.17 & -.18 & $.87 * *$ & -.13 & -- \\
\hline
\end{tabular}

$* p<.05 ; * * p<.01$ 


\section{Main Analyses}

Based on preliminary research on mindfulness and coping (Palmer \& Rodger, 2009; Weinstein, Brown \& Ryan, 2009), we hypothesized that brief mindfulness training would encourage greater use of mindful coping strategies and diminished use of emotionfocused coping strategies among participants relative to an alternate training or control group, and that mindfulness training would further be associated with an attenuated stressor response, as assessed by both self-report measures of anxiety and affect, and physiological indices of cortisol and cytokine response.

Group differences in endorsement of coping strategies. A 3 (Training type: mindfulness vs. problem-solving vs. control) x 2 (Task type: stressor vs. control) between-subjects MANOVA was conducted to assess differences in the use of coping strategies among participants as a function of the training group and Session 2 task condition. Results of this analysis indicated that the use of coping strategies reported by participants during the stressor or control task did not differ as a function of training group, $F(8,156)=1.93, p=.09^{7}$, partial $\eta^{2}=.091$, power $=.80$ or task type, $F(4,78)=$ $1.34, p=.26$, partial $\eta^{2}=.064$, power $=.40$. These non-significant main effects indicated that the coping strategies endorsed by individuals did not differ as a function of participation in the mindfulness, problem-solving or active control training groups. Likewise, the use of coping strategies did not significantly vary among individuals, regardless of whether they completed the stressor (speech) or control (writing) task upon returning for the second laboratory session. Interactions between conditions were all

\footnotetext{
${ }^{7}$ The assumption of homogeneity of covariance was met in the present analysis (Box's $\mathrm{M}=55.71, p=$ $.54)$; as such, Wilk's $\lambda$ is reported for all $F$ tests.
} 
non-significant in the present investigation $(p>.10)$, suggesting that the effect of training condition on coping responses among participants did not vary as a function of the type of task completed (speech vs. writing task). See Table 3.2.3 for a summary of group means and standard deviations. 
Table 3.2.3

Summary of Descriptive Statistics for Coping Strategies Endorsed as a Function of

Training Type (Mindfulness, Problem-Solving or Control) and Task Type (Stressor vs.

Control) $(N=107)$

\begin{tabular}{|c|c|c|c|c|c|}
\hline $\begin{array}{l}\text { Training Type } \\
\text { (Condition 1) }\end{array}$ & $\begin{array}{c}\text { Task Type } \\
\text { (Condition 2) }\end{array}$ & $n$ & Coping Strategy & $M$ & $S D$ \\
\hline \multirow[t]{8}{*}{ Mindfulness } & Stressor & 18 & Problem-focused & 6.57 & 1.75 \\
\hline & & & Emotion-focused & 15.97 & 3.31 \\
\hline & & & Avoidant & 4.36 & 1.21 \\
\hline & & & Mindful & 2.03 & .49 \\
\hline & Control & 17 & Problem-focused & 6.89 & 1.36 \\
\hline & & & Emotion-focused & 16.08 & 3.93 \\
\hline & & & Avoidant & 4.43 & 1.09 \\
\hline & & & Mindful & 1.95 & .57 \\
\hline \multirow[t]{8}{*}{ Problem-Solving } & Stressor & 18 & Problem-focused & 6.97 & 1.68 \\
\hline & & & Emotion-focused & 15.95 & 3.01 \\
\hline & & & Avoidant & 4.31 & 1.10 \\
\hline & & & Mindful & 2.34 & .34 \\
\hline & Control & 17 & Problem-focused & 6.95 & 1.70 \\
\hline & & & Emotion-focused & 13.95 & 3.59 \\
\hline & & & Avoidant & 4.70 & .81 \\
\hline & & & Mindful & 2.20 & .44 \\
\hline \multirow[t]{8}{*}{ Control (no training) } & Stressor & 16 & Problem-focused & 8.04 & 1.59 \\
\hline & & & Emotion-focused & 17.80 & 5.45 \\
\hline & & & Avoidant & 4.65 & .74 \\
\hline & & & Mindful & 2.22 & .59 \\
\hline & Control & 21 & Problem-focused & 7.13 & 1.98 \\
\hline & & & Emotion-focused & 13.94 & 4.62 \\
\hline & & & Avoidant & 4.44 & 1.05 \\
\hline & & & Mindful & 2.26 & .59 \\
\hline
\end{tabular}


Group differences in post-task state anxiety and affect. A second $3 \times 2$ between-subjects MANOVA was performed to assess differences in psychological variables (state anxiety, positive and negative affect) following the stressor or control task. As in the previous analysis, the assumption of homogeneity of covariance between groups was met, Box's $\mathrm{M}=34.01, p=.43$; all $F$ values were therefore tested and reported using Wilk's $\lambda$ criterion.

An examination of main effects indicated that the task type (stressor vs. control task) was significant, $F(3,79)=4.87, p<.01$, power $=.89$, accounting for $15.6 \%$ of the variance in the linear combination of psychological response variables. Follow-on analyses indicated that participants in the stressor condition reported greater state anxiety $(M=2.73, S D=.60)$ relative to those in the control condition $(M=2.29, S D=.45)$, $F(1,81)=13.67, p<.01$, partial $\eta^{2}=.144$, power $=.96$, as well as greater negative affect (stressor $M=1.58, S D=1.27$; control $M=.67, S D=.87$ ) post-task, $F(1,81)=13.85, p<$ .01 , partial $\eta^{2}=.146$, power $=.96$. Although mean positive affect ratings were slightly higher among participants in the control task condition, this relationship did not reach statistical significance $(p=.36)$. See Figure 3.2.1 for a summary of group means and standard errors.

In contrast to the effects of the task type, the Session 1 training condition was not significantly associated with differences in psychological variables following the stressor or control task $, F(6,158)=.68, p=.67$, partial $\eta^{2}=.025$, power $=.26$. More specifically, the length and/or content of this training was not sufficient to elicit changes in state anxiety $(p=.39)$, positive affect $(p=.54)$ or negative affect $(p=.28)$ following an acute stressor task. 


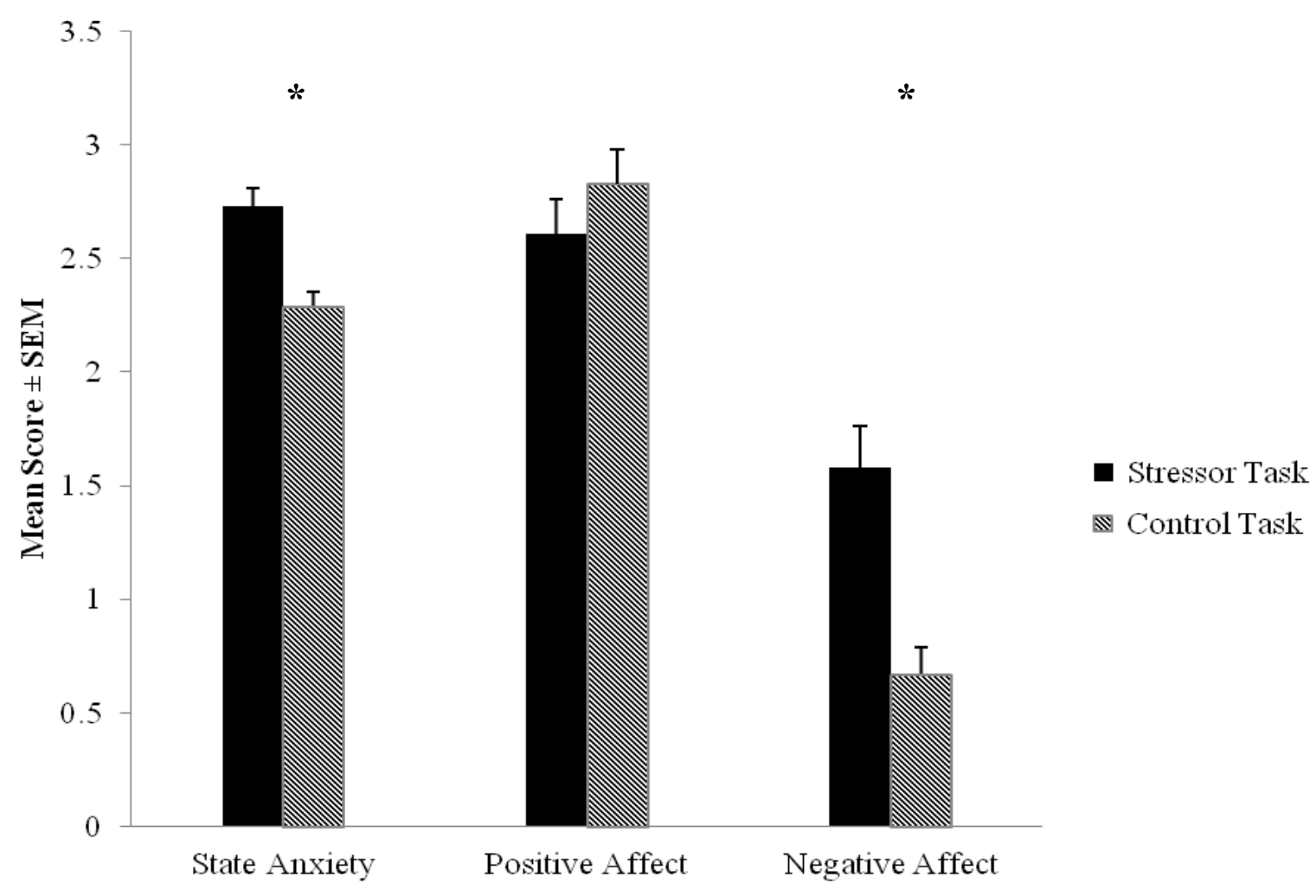

Post-Task Psychological Variables

Figure 3.2.1. Mean ( \pm SEM) State Anxiety, Positive Affect and Negative Affect Ratings as a Function of Task Type.

Group differences in cortisol and cytokine response. In addition to identifying group differences in coping and post-task anxiety and affect, a primary goal of this study was to determine if brief mindfulness training promoted lower levels of baseline cortisol among participants, and to determine if this training was associated with lower levels of circulating cortisol and cytokines during a subsequent acute stressor challenge. 
A preliminary 3 (mindfulness vs. problem-solving vs. control training) x 2 (stressor vs. control task) between-subjects factorial MANOVA was conducted to determine if training or task type were associated with differences in baseline cortisol and cytokine response among participants. Results of this preliminary analysis indicated that neither training condition, $F(8,152)=.92, p=.50$, partial $\eta^{2}=.046$, power $=.42$ nor assignment to the stressor or control task, $F(4,76)=.59, p=.68$, partial $\eta^{2}=.030$, power $=.19$ predicted differences in baseline cortisol or cytokine levels among participants.

A series of 3 (mindfulness vs. problem-solving vs. control training) x 2 (stressor vs. control task) x 3 (assessment time) mixed repeated measures ANOVAs were then used to identify any changes in cortisol and cytokines over time as a function of training and/or task group. Training and task condition were entered as between-subjects factors, and cortisol, TNF $\alpha$, IL-6 and IL-10 values were entered as repeated measures (withinsubjects) factors, respectively. All hormones were assessed at three time points: at baseline, following the task preparation period (anticipation measure) and 15 minutes post-task.

Cortisol. An examination of Omnibus effects revealed that the main effect of time of assessment was non-significant, $F(2,79)=.27, p=.76$, partial $\eta^{2}=.007$, power $=.09$. Similarly, the interaction between training condition and time of assessment was non-significant, $F(4,158)=1.35, p=.25$, partial $\eta^{2}=.033$, power $=.41$, indicating that cortisol response did not vary over time irrespective of whether individuals had completed brief mindfulness training or participated in an alternate training group (e.g., problem-solving or control). As expected, however, a significant interaction was observed between task type and time of assessment, $F(2,79)=.5 .70, p<.01$, partial $\eta^{2}=$ 
.126 , power $=.85$. A follow-on analyses indicated that plasma cortisol increased among participants in the stressor condition $\left(M_{\text {diff }}=3.16\right)$, and decreased among participants in the control condition $\left(M_{\text {diff }}=-1.44\right)$ from baseline to post-task, suggesting that the speech task was indeed effective in eliciting a stressor response. See Figure 3.2.2 for a summary of the interaction between task condition and time of assessment. 


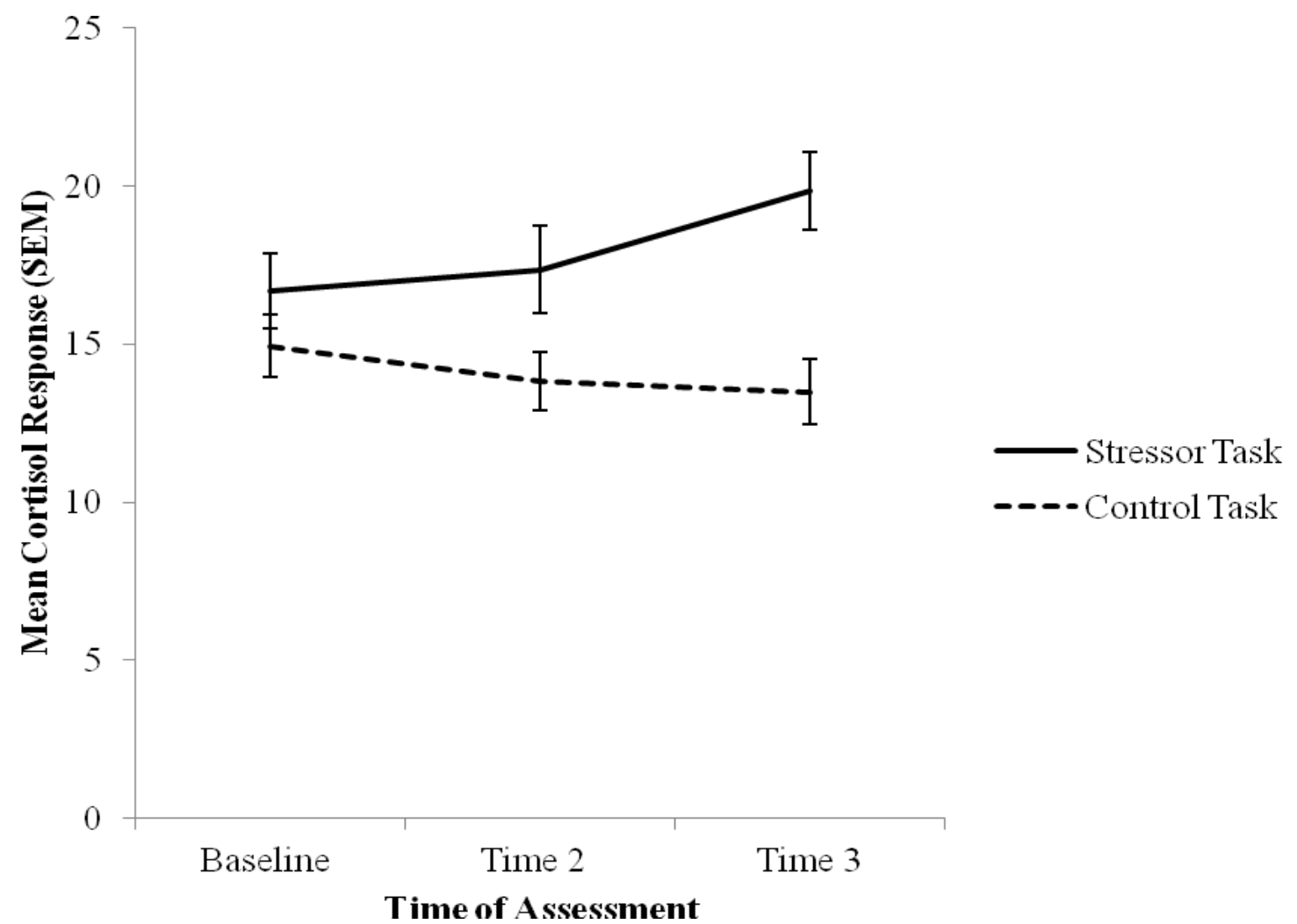

Figure 3.2.2. Mean ( \pm SEM) Cortisol Response as a Function of Time of Assessment and Task Type.

TNF $\boldsymbol{\alpha}$. A second analysis was performed to assess changes in TNFa from baseline to post-task, as a function of training and task groups. Omnibus effects for the main effect of training condition, $F(2,77)=.09, p=.91$, partial $\eta^{2}=.002$, power $=.06$, the main effect of task type, $F(1,77)=.32, p=.57$, partial $\eta^{2}=.004$, power $=.09$, and time of assessment, $F(2,76)=.12, p=.89$, partial $\eta^{2}=.003$, power $=.07$ were all nonsignificant. Evidently, $\mathrm{TNF} \alpha$ did not vary across assessment periods (from baseline to post-task), regardless of whether individuals had participated in a stressor or control task, 
or had completed brief mindfulness training versus alternate training (e.g., problemsolving or control). Similarly, the two-way interactions between training condition and time of assessment, $F(4,152)=1.28, p=.28$, partial $\eta^{2}=.032$, power $=.39$, and task type and time of assessment, $F(4,152)=1.28, p=.28$, partial $\eta^{2}=.032$, power $=.39$ were not significant in the present study. These results suggest that the effect of training group on TNF $\alpha$ did not vary among individuals as a function of the type of task completed.

Interleukin-6. A third mixed repeated measures ANOVA was examined to determine whether the pro-inflammatory cytokine IL-6 varied from baseline to post-task as a function of training or task condition. As in the previous analyses, Session 1 training condition (mindfulness vs. problem-solving vs. control training) and Session 2 task condition (stressor vs. control task) were entered as between-subjects factors, and time of assessment was investigated as a within-subjects, repeated measure factor.

An examination of Omnibus effects indicated that the main effects of training condition, $F(2,77)=.07, p=.93$, partial $\eta^{2}=.002$, power $=.06$, task condition, $F(1,77)$ $=.04, p=.85$, partial $\eta^{2}=.001$, power $=.05$ and time of assessment, $F(2,76)=1.72, p=$ .19 , partial $\eta^{2}=.043$, power $=.35$ were non-significant. Similarly, an analysis of two and three-way interactions between condition and time of assessment were non-significant ( $p$ 's $>.12$ ), suggesting that IL-6 response among participants was not systematically associated with training groups or task type in the present study.

Interleukin-10. A final mixed repeated measures ANOVA was calculated to determine if brief mindfulness training was associated with differences in IL-10 across 
assessment periods. As in the previous analyses, the main effect of training was not associated with IL-10 response, $F(2,77)=.11, p=.90$, partial $\eta^{2}=.003$, power $=.13$, and no significant differences emerged as a function of task type, $F(1,77)=.65, p=.42$, partial $\eta^{2}=.008$, power $=.13$ or time of assessment, $F(2,76)=1.70, p=.19$, partial $\eta^{2}=$ .043 , power $=.35$. All tests of two and three-way interactions between grouping variables additionally failed to reach statistical significance $(p>.14)$, suggesting that IL10 was not affected by training or task type across the present assessment period.

\section{Facets of trait mindfulness as predictors of depressive symptoms and}

internalized stigma. To replicate the relationships observed in our previous investigation, a series of multiple regression analyses were performed to determine if specific trait mindfulness subscales predicted depressive symptoms and internalized stigma among participants in the present sample. Results of the first analysis indicated that facets of trait mindfulness significantly predicted depressive symptoms among young adults, $F(5,101)=5.16, p<.01, \mathrm{R}^{2}{ }_{\text {adj }}=.164$. Specifically, higher endorsement of nonreacting to experience, $\mathrm{B}=-2.11, S E=1.01, t=-2.09, p=.04$ and non-judging of experience, $\mathrm{B}=-1.60, S E=.70, t=-2.28, p=.03$ were associated with lower depressive symptomatology among participants. Likewise, trait mindfulness subscales significantly predicted internalized stigma in the present sample, $F(5,101)=11.19, p<.01, \mathrm{R}^{2}$ adj $=$ .325. An analysis of specific parameters revealed that this effect was driven by nonjudging of experience, $\mathrm{B}=-1.12, S E=.27, t=-4.13, p<.01$. As anticipated, the direction of this effect indicated that individuals who did not judge themselves for the thoughts and emotions they experienced reported significantly fewer self-stigmatizing views towards depressive symptoms. 
Facets of trait mindfulness as predictors of coping strategies endorsed. In

addition to evaluating the effectiveness of our brief mindfulness intervention, a primary goal of the present study was to determine the extent to which specific facets of trait mindfulness predicted coping, psychological and physiological responses to an acute stressor task. To investigate the contribution of these facets, a series of multiple regression analyses were conducted. In the first analysis, facets of trait mindfulness were examined as continuous predictor variables, and coping strategies (problem-focused, emotion-focused, avoidant and mindful) were examined as dependent variables.

Results of this analysis indicated that coping strategies in the present sample were significantly predicted by non-reacting to experience, $F(4,78)=3.04, p=.02$, partial $\eta^{2}$ $=.135$, power $=.78$, observing inner experience, $F(4,78)=2.65, p=.04$, partial $\eta^{2}=$ .120 , power $=.72$, describing and labelling, $F(4,78)=4.18, p<.01$, partial $\eta^{2}=.177$, power $=.91$, and non-judgment of thoughts and feelings, $F(4,78)=.4 .99, p<.01$, partial $\eta^{2}=.204$, power $=.95$. An examination of specific parameters indicated that observing inner experience, $\mathrm{B}=.24, t=2.93, p<.01$, partial $\eta^{2}=.096$, and non-judgment of thoughts and feelings, $\mathrm{B}=.15, t=2.40, p=.02$, partial $\eta^{2}=.067$ positively predicted the use of mindful coping strategies among participants, while the tendency to observe inner experience, $\mathrm{B}=.58, t=1.92, p<.01$, partial $\eta^{2}=.043$ and the ability to describe and label (e.g., articulate) thoughts and feelings, $\mathrm{B}=.79, t=3.37, p<.01$, partial $\eta^{2}=.123$ predicted the use of problem-focused coping strategies during a stressor task. Moreover, the tendency to suspend judgment for thoughts and feelings predicted a decline in the use of emotion-focused coping strategies among participants, $\mathrm{B}=-1.34, t=-3.02, p<.01$, partial $\eta^{2}=.101$. Results of this analysis support a facet approach to the study of trait 
mindfulness and coping, suggesting that theorized facets of mindfulness differentially predict coping efforts among young adults. In particular, the ability to mindfully observe experiences, and to suspend self-judgment as thoughts and feelings are experienced appear to predict greater endorsement of mindful and problem-based coping efforts, and to discourage the use of emotion-focused coping strategies (e.g., rumination) in response to potentially stressful situations.

A second series of multiple regression analyses was conducted to determine if the use of various coping strategies predicted state anxiety, affect, and subsequent cortisol response among participants. Results of these analyses indicated that coping strategies significantly predicted state anxiety, $F(4,92)=4.25, p<.01$ and negative affect $F(4$, $92)=3.00, p=.02$. In particular, the use of emotion-focused coping strategies predicted increased anxiety $\mathrm{B}=.05, S E=.02, t=2.76, p=.01$ and negative affect, $\mathrm{B}=.06, S E=$ $.02, t=3.79, p<.01$. Coping strategies were not found to predict cortisol response among participants in the stressor condition, $F(4,41)=.86, p=.50, n s$ or the control condition, $F(4,36)=1.63, p=.19$, ns. These results suggest that although the use of emotion-focused coping strategies predicted higher levels of subsequent state anxiety and negative affect among participants, the use of specific types of coping strategies did not influence neuroendocrine response during an acute stressor task.

\section{Facets of trait mindfulness as predictors of post-task state anxiety and affect.}

A second multiple regression analysis assessed the utility of trait mindfulness facets in predicting state anxiety, positive and negative affect, following the laboratory task. These analyses indicated that trait mindfulness facets marginally and inversely predicted state anxiety among participants, $F(5,91)=2.13, p=.07, \mathrm{R}_{\text {adj }}^{2}=.055$, but did not 
significantly predict post-task positive affect, $F(5,91)=1.12, p=.36, n s$ or negative affect, $F(5,91)=.82, p=.54, n s$ in the present investigation.

\section{Facets of trait mindfulness as predictors of cortisol and cytokine response.}

Although earlier research has associated trait mindfulness with diminished cortisol response following a stressor (e.g., Brown, Weinstein \& Creswell, 2012), limited information is available as to the specific components or facets of trait mindfulness that drove this effect. To investigate these relationships, a series of multiple regression analyses were performed to determine whether facets of trait mindfulness predicted 1) differences in baseline cortisol and cytokine levels, and 2) changes in cortisol and cytokines from baseline to post-task.

Trait mindfulness facets and baseline cortisol and cytokines. A series of multiple regression analyses were conducted to determine if facets of trait mindfulness predicted baseline cortisol and cytokine values among young adults. As expected, trait mindfulness scores on the FFMQ significantly predicted baseline cortisol levels among participants, $F(5,81)=2.27, p=.05$. The regression model including these facets accounted for $12.3 \%$ of the variance in baseline cortisol. An analysis of specific subscales revealed that the "acting with awareness", $\mathrm{B}=-4.44, t=-3.03, p=.003$ and "non-judging of experience", $\mathrm{B}=-2.03, t=-2.00, p=.05$ subscales of the FFMQ drove this effect. More specifically, the direction of these effects indicated that greater awareness of thoughts and feelings and lower self-judgment were associated with lower baseline cortisol levels among participants. Contrary to our expectations, trait mindfulness did not predict significant differences in baseline TNF $\alpha(p=.67)$, IL-6 $(p=$ $.62)$ or IL-10 $(p=.33)$ in the present sample. 


\section{Trait mindfulness facets and differences in cortisol and cytokine response.}

Multiple regression was used to determine whether the five facets of trait mindfulness assessed by the FFMQ (non-judgment of thoughts and feelings, observing inner experience, acting with awareness, describing and labelling, and non-reacting to inner experience) significantly predicted cortisol response from baseline to post-task. In the first analysis, the five subscales of the FFMQ were entered in to the model as continuous predictors, and a (from baseline to post-task). Results of this analysis indicated that the model including facets of trait mindfulness was significant, $F(5,80)=2.20, p=.05$, and accounted for $12.1 \%$ of the variance in cortisol response across participants ${ }^{8}$. An examination of specific parameters revealed that non-reacting to experience drove this effect, $\mathrm{B}=-2.37, S E=.96, t=-2.47, p=.02, s r=.259$. As hypothesized, the direction of this effect indicated that lower reactivity to experiences predicted diminished cortisol response among participants from baseline to post-task. No other facets of trait mindfulness significantly predicted cortisol response in the present investigation ( $p$ 's $>$ $.10)$.

Subsequent multiple regression analyses were conducted to determine if facets of trait mindfulness predicted changes in cytokines from baseline to post-task. These analyses revealed that trait mindfulness facets did not significantly predict changes in $\mathrm{TNF} \alpha, F(5,81)=1.36, p=.25, R_{a d j}^{2}=.021, \mathrm{IL}-6, F(5,79)=.53, p=.75, R_{a d j}^{2}=.032$, or IL-10, $F(5,79)=1.31, p=.27, R_{a d j}^{2}=.018$ from baseline to post-task.

\footnotetext{
${ }^{8}$ Effects were examined separately for participants in both the stressor and control conditions, and the direction and magnitude of effects was consistent across both groups.
} 


\section{Discussion and Conclusions}

Although trait mindfulness and mindfulness training programs (e.g., MBSR) appear to be effective in improving both physical symptoms (e.g., pain) and psychological symptoms (e.g., depression, anxiety) across various clinical and nonclinical populations (e.g., Brown, Weinstein \& Creswell, 2012; Grossman, Niemann, Schmidt \& Walach, 2004; Kabat-Zinn, 1990), little is known about the ways in which mindfulness influences these outcomes. To extend this body of research, the purpose of the present investigation was to examine predictive relationships between specific facets of trait mindfulness, depressive symptoms, coping strategies, and internalized stigma for depressive symptoms. A further goal of this study was to determine if brief mindfulness training was effective in promoting the use of active coping strategies in response to stressors, and to assess whether this intervention was effective in mitigating psychological (e.g., perceived anxiety, positive and negative affect), and physiological responses to a social stressor (i.e., changes in cortisol and cytokine reactivity). Since depressive symptoms and internalized stigma tend to be characterized (and perpetuated) by a repetitive focus on negative thoughts and emotions, as well as tendencies towards negative self-reflection and self-evaluation (Beck, Rush, Shaw \& Emery, 1979; River \& Holmes, 1998; Rüsch, Corrigan, Todd \& Bodenhausen, 2010), we hypothesized that nonjudgment and non-reactivity to experiences would constitute specific facets of mindfulness that would predict reductions in depression and self-stigma.

Supporting our previous research, trait mindfulness was inversely associated with both depressive symptoms and internalized stigma among students in the present sample. An analysis of mindfulness facets confirmed that non-judgment of inner experiences 
(thoughts and emotions) represented a specific aspect of trait mindfulness that accounted for this effect. Individuals who tended to accept their thoughts and reactions to events without judging them reported fewer and/or less severe symptoms of depression, and fewer self-stigmatizing views relative to those scoring low on this facet of mindfulness. This finding was consistent with our expectations, and suggests that mindfulness skills centered on acceptance and non-judgment might be especially instrumental in reducing vulnerability to depressive illness.

The coping strategies used by participants during the study task were related to trait mindfulness. Specifically, observing and non-judgment of inner experiences, and the ability to describe and label (e.g., articulate) thoughts and feelings predicted greater use of mindful and problem-focused coping strategies among participants, while the tendency to suspend judgment for thoughts and feelings predicted a decline in the use of emotion-focused coping strategies during a stressor task. Results of these analyses support a facet approach to the study of trait mindfulness and coping, suggesting that theorized facets of mindfulness differentially predict coping efforts among young adults, and that the suspension of self-judgment in particular may help to discourage the use of emotion-focused coping strategies in response to potentially stressful situations.

As expected, the use of various coping strategies predicted post-task state anxiety and affect among participants. In particular, the use of emotion-focused coping strategies predicted greater anxiety and negative affect among individuals following the stressor task. Coping strategies were not found to predict the cortisol response among participants in either the stressor condition or the control condition. It seems that 
although the use of emotion-focused coping strategies predicted higher levels of subsequent state anxiety and negative affect, the use of specific coping strategies did not.

Prior research evaluating 8-week Mindfulness-Based Stress Reduction (MBSR) programs has shown that such interventions are effective in reducing physical and psychological symptoms, including anxiety, pain and depression (e.g., Segal, Williams \& Teasdale, 2002), improving general health and functioning across diverse populations (e.g., Grossman, Niemann, Schmidt \& Walach, 2004) and improving immune response (Davidson, Kabat-Zinn, Schumacher, Rosenkranz, Muller, Santorelli, et al., 2004). Despite these benefits, however, programs can be costly to administer, and require an intensive time commitment on the part of clients seeking treatment. In addition, although these interventions are increasingly being offered through hospital and private practice settings in Ontario, these programs may not be widely available in different regions (or offered only a few times each year), and as such may not be accessible to many individuals who would benefit from treatment. In light of these limitations, increasing emphasis has been placed on the need to develop shorter mindfulness programs in order to encourage a greater number of individuals to access services (e.g., Zeidan, Johnson, Gordon \& Goolkasian, 2010). As such, one goal of the present study was to evaluate the efficacy of a brief mindfulness session in reducing distress among university students, and to determine if a short mindfulness intervention was associated with changes in cytokine response in the context of an acute stressor. This session included mindful meditation and awareness exercises, adapted from longer and empirically validated MBSR and MBCT programs (Segal, Williams \& Teasdale, 2002). Results of the present study indicated that the social stressor task prompted significant increases in cortisol 
response, state anxiety and negative affect among participants. Contrary to our expectations, however, no differences in cortisol or cytokine response were apparent in the present study as a function of brief mindfulness training.

There are several possible explanations for this lack of an effect between our mindfulness intervention and psychological and neuroendocrine indices. One possibility is that the single 90 -minute session may have been too brief to affect change in participants' thinking or experiential processing. Although we included homework activities designed to help individuals consolidate and practice mindfulness exercises during the week between the training session and the laboratory session, it is likely that students varied in their commitment and level of engagement in these tasks. As such, mindfulness concepts may not have been sufficiently processed or integrated among participants to promote observable changes in psychological response (e.g., state anxiety, affect) or physiological response (changes in cortisol or cytokines) post-task. In addition, past research (e.g., Zeidan, Johnson, Gordon \& Goolkasian, 2010) has demonstrated that short mindfulness meditation sessions and mindfulness priming exercises might have short-term effects on well-being, but that these effects tend to deteriorate over time if not sustained through practice and consolidation. It is possible, therefore, that any immediate benefits of the mindfulness training may have deteriorated in the one week interval between sessions, particularly if students were not motivated to practice or further process the information and techniques learned.

Finally, to extend upon previous literature on mindfulness and stressor response, the contribution of specific facets of trait mindfulness to psychological and physiological reactivity to stressors was assessed. Results of the present study indicated that higher 
trait mindfulness scores were associated with diminished state anxiety across participants post-task. Furthermore, awareness of thoughts and emotions predicted significantly lower resting/baseline cortisol levels among participants, while high nonreactivity to experience (e.g. the ability to recognize and accept thoughts and emotions without emotionally engaging) predicted a diminished cortisol response from baseline to post-task. These results suggest that different facets of mindfulness might be influential at different stages of the stress and coping process; in particular, it appears that the specific ability to stay in touch with thoughts and emotions may help to regulate resting cortisol levels, while the ability to limit emotional engagement may help to attenuate cortisol response during periods of acute stress. Thus, the present study suggests that trait mindfulness plays an important role in aspects of stress and coping processes, and that further evaluating the specific contribution of different facets of trait mindfulness may provide additional insight into causal mechanisms and pathways between mindfulness, stress, coping and well-being.

\section{General Discussion}

In spite of growing research and accessibility of treatment, depression continues to represent a leading cause of global disease and disability (WHO, 2007), and additional work is needed to diminish the prevalence of depressive illness and to mitigate the negative consequences of this illness at both an individual and societal level. The concept of mindfulness has received increasing attention in this regard, and has been purported to enhance coping ability and confer resistance to depression, stress and illness by encouraging individuals to attend actively to their surroundings as well as physical and emotional responses to events in a non-judgmental way (Brown \& Ryan, 2006; 
Kabat-Zinn, 1990; Segal, Williams \& Teasdale, 2002). Although mindfulness has been linked to well-being, little is known about the mechanisms through which mindfulness confers mental and physical health benefits, and differences in operational definitions, research methodology, and measures used to assess trait mindfulness often do not allow for synthesis or comparisons between studies (e.g., Bishop et al., 2004; Brown \& Ryan, 2004).

To build upon existing literature on mindfulness and well-being, the present research sought to assess the relationship between mindfulness and self-appraisals, and to examine how mindfulness influences the types of strategies individuals use for coping with stressful situations (Studies 1 and 2). Extending upon previously observed associations (e.g., Kabat-Zinn, 1990), we observed that higher levels of trait mindfulness predicted greater endorsement of problem-focused coping strategies and enhanced perceptions of control over potentially stressful scenarios, and was negatively associated with the endorsement of emotion-focused coping styles. This supports previous research (e.g., Weinstein, Brown \& Ryan, 2009), which has largely attributed the negative relationships observed between trait mindfulness and emotion-focused coping to an enhanced capacity within mindful individuals to limit immediate, reflexive emotional responses to events. It is widely theorized that the present-focused nature of mindfulness may encourage individuals to "disable" automatic pilot tendencies (e.g., automatic emotional responses), and to focus instead on actively accepting and processing their actions and responses to events (e.g., Kabat-Zinn, 1990; Segal, Williams \& Teasdale, 2002). If individuals are successfully able to live in the moment this way, they may recognize that their responses are not predetermined or constrained by habit; rather, there 
are choices available with respect to the thoughts and actions they may choose to take in response to their environment. According to this interpretation, mindfulness might facilitate adaptive coping and enhanced perceptions of control across various situations, as individuals are able to suspend negative thinking and emotional responses, and focus instead on moment to moment processing and the selection of appropriate thoughts and behaviours.

Interestingly, the relationship that emerged between trait mindfulness and avoidant coping was more complex than originally anticipated. While mindfulness is predicated on active, sustained awareness and engagement in the present moment, and has been associated with improved health and well-being, the use of avoidant coping strategies has been conceptualized as a means of physically, mentally or emotionally disengaging from encounters, and long-term use of these strategies has been associated with increased anxiety, depression, and distress (e.g., Fledderus, Bohlmeijer \& Pieterse, 2010; Lazarus \& Folkman, 1984; Seiffge-Krenke \& Klessinger, 2000). Based upon this literature, we initially expected that trait mindfulness would inversely predict avoidant coping tendencies among young adults. Across the majority of the present studies, however, this relationship was not supported; rather, positive associations were observed between mindfulness and the endorsement of avoidant coping techniques.

This seemingly counter-intuitive result may be explained by the tendency of mindful individuals to hold situations at arm's-length and suspend automatic reactions to events (i.e., de-centering). Since mindfulness involves focusing on moment-to-moment reactions and sensations rather than focusing upon long-term resolution or control over stressors, it is possible that avoidant coping strategies (e.g., cognitive distraction or 
engagement in other activities) might offer a temporary reprieve from any emotional distress elicited by these stressors. The cognitive space afforded by these avoidant techniques might be especially beneficial for highly mindful individuals, allowing them the time necessary to process thoughts, sensations, physical and emotional responses to stressors prior to acting upon them. This interpretation fits with the relationships observed in the present research between trait mindfulness and the use of avoidant coping strategies, and suggests that such strategies may be endorsed (and even beneficial in the short-term) among mindful individuals, allowing them time to acknowledge and process new information and responses to events.

In light of strong evidence that depressive symptoms may be exacerbated by internalized stigma for illness (e.g., Livingston \& Boyd, 2010), a second aim of this research was to determine if trait mindfulness inhibits the internalization of stigma, thereby conferring resistance to depressive symptoms. We hypothesized that trait mindfulness would predict reductions in internalized stigma, and that this reduction, in turn, would predict a lower frequency and severity of depressive symptoms. We further theorized that facets of non-judgment and acceptance would be particularly instrumental in discouraging internalized stigma among participants, thereby representing one pathway or mechanism through which mindfulness influences stigma and the development of depressive symptoms.

In line with our expectations, results of Studies 1 and 2 demonstrated that trait mindfulness predicted lower rates of internalized stigma across both student and community samples, which in turn predicted lower depressive symptomatology. When mindfulness was further assessed as a multi-faceted trait (Studies 3.1 and 3.2), non- 
judgment of thoughts and feelings emerged as a unique predictor of internalized stigma, indicating that the practice of non-judgment represents a specific component of trait mindfulness that may discourage the internalization of negative stereotypes and subsequent exacerbation of depressive symptoms. Extending from these results, mindfulness programs developed for use with depressed individuals might benefit from emphasizing non-judgment and self-acceptance in order to reduce the likelihood that individuals will adopt self-stigmatizing views stemming from their symptoms and experiences.

Although trait mindfulness has been associated with numerous health benefits, self-reported mindfulness may not translate to the practice of mindful behaviours across different situations. To investigate the relationship between trait measures and behaviour, the present research explored the concordance between self-reported trait mindfulness and the endorsement of mindful coping behaviours, and sought to identify ways in which mindful coping efforts vary, both across individuals and across different types of stressful situations (e.g., personal, financial, academic/workplace stressors). Across all studies, greater trait mindfulness predicted a decline in the use of emotionfocused coping strategies; this pattern was observed in terms of general coping styles (Study 1), in response to diverse stressors including interpersonal conflicts and academic or workplace stressors (Study 2), and in response to an acute stressor task (Study 3). The activation of mindful behaviours (e.g., mindful coping) was highly associated with control appraisals and the use of problem-focused coping strategies, and negatively correlated with depressive symptoms and distress appraisals across both student and community samples (Study 2). 
It had been expected that trait mindfulness would be positively associated with mindful coping, such that highly mindful individuals would tend to employ mindfulnessbased techniques (e.g., de-centering from emotional responses and focusing on the present moment) in response to stressors. This hypothesis was partially supported. Unidimensional trait mindfulness scores (as assessed on the MAAS) were not predictive of mindful coping efforts in Study 2; however, specific facets of trait mindfulness were found to predict the use of mindfully-based coping strategies (Study 3). In particular, the ability to mindfully observe experiences and suspend judgment of thoughts and feelings predicted greater endorsement of mindful and problem-based coping efforts, and was negatively associated with the use of emotion-focused coping strategies in response to potentially stressful situations. Taken together, these results suggest that although aspects of attention and awareness in themselves are not necessarily sufficient to activate adaptive coping behaviour, other mindfulness-based skills (e.g., the practice of nonjudgment) might encourage the deployment of active and problem-focused coping responses.

A further goal of this study was to determine if mindfulness influenced physiological changes (i.e., cortisol and cytokine activity) in response to an acute stressor, and to identify whether mindfulness could be enhanced among individuals to elicit these effects. Specifically, we sought to determine whether trait mindfulness was associated with decreased physiological responses following a stressor challenge (Study 3.1), and to determine if brief mindfulness training would encourage more benign physiological responses to stressors (Study 3.2). 
Trait mindfulness. Based on previous literature (e.g., Brown \& Ryan, 2003), we hypothesized that trait mindfulness would negatively predict depressive symptoms and the use of emotion-focused coping strategies across participants. As anticipated, high trait mindfulness was associated with lower endorsement of emotion-focused coping strategies, lower rates of depressive symptoms and internalized stigma, and lower anxiety and negative affect following a stressful task (Studies 3.1 and 3.2). Furthermore, an analysis of mindfulness facets indicated that mindful observing (Study 3.1) and awareness (Study 3.2) predicted lower baseline cortisol among participants, while nonreacting to experience predicted a diminished cortisol response during participation in a stressful task (Study 3.2). These results suggest that specific mindfulness skills may contribute differentially to the stressor response, with some skills contributing to lower resting cortisol rates, and other facets contributing to a diminished stressor response as stressful tasks are processed. Contrary to our expectation, however, trait mindfulness did not predict differences in cytokine response across the present studies.

Mindfulness training. It will be recalled that traditional 8-week Mindfulnessbased Stress Reduction and Mindfulness-based Cognitive Therapy programs have been associated with improvements in appraisal, coping, anxiety and depressive symptomatology (see Kabat-Zinn, 1990; Segal, Williams \& Teasdale, 2002). These programs, however, require a significant time commitment on the part of participants, and the typical cost of these programs may be prohibitive to individuals not being seen within a clinical treatment context. Given these potential barriers to participation in traditional 8-week programs, we sought to evaluate the effectiveness of a shorter 
mindfulness intervention in improving affect and mitigating cortisol response following an acute stressor task.

In this brief, 90-minute intervention, participants completed a series of mindfulness-based exercises (e.g., mindful meditation, awareness and breathing exercises), problem-solving (e.g., practicing and identifying analytical problem-solving techniques), or received no training (e.g., an active control session which focused on identifying meaningful personal activities). Given the brief nature of the mindfulness and problem-solving training, participants were given a homework assignment to complete each day leading up to the second study session designed to consolidate and reinforce the information and techniques learned within the training session. An analysis of responses at the second session, however, indicated that our brief mindfulness training was not effective in diminishing anxiety, and was not associated with a diminished cortisol or cytokine response following a stressor task.

This lack of a training effect may have been observed for a number of reasons. First, although the goal of the present study was to determine if a brief training session was capable of eliciting beneficial effects upon exposure to a stressor task one week later, the session offered may have simply been too brief to affect change in participant responses, and there may be a "minimum effective dose" or threshold for training that was not met through this short intervention. In addition, the assigned homework activities were designed to allow participants time to practice and engage with the concept of mindfulness (thus more closely approximating longer 8-week programs). Although these exercises may be extremely beneficial for individuals who are motivated to learn and apply these techniques, it is possible that students may not have been as 
motivated to complete these exercises, especially in the absence of clinical symptoms. Furthermore, it is possible that any positive effects of the mindfulness training diminished over the one-week interval between sessions, and that longer sessions may be needed to maintain beneficial effects over time. Future research might improve on this design by offering longer training sessions, or by implementing additional controls (e.g., the use of personal recording devices) to better monitor participation and engagement in homework assignments following brief mindfulness training. Despite the lack of observed associations between our mindfulness intervention and stress and coping responses in the present investigation, evaluations of brief mindfulness programs are warranted, as these might offer cost-effective and increasingly accessible options for individuals to develop mindfulness skills.

\section{Limitations}

The present research contributes to the existing body of literature on mindfulness, stress and well-being by exploring potential pathways and mechanisms through which mindfulness influences stress and depressive illness among young adults. There are, however, limitations to this program of research that should be addressed.

As with many trait investigations, Studies 1 and 2 employed a cross-sectional and correlational design. Although theoretically sound and empirically-based predictive relationships were observed between trait mindfulness, endorsement of coping strategies, depressive symptoms and internalized stigma, this type of design does not permit causal inferences to be drawn, and it is possible that bi-directional or reciprocal relationships exist between mindfulness, coping, stigma and depressive symptoms. A large body of personality literature posits that traits are stable and enduring over time, providing a 
dispositional framework from which individuals approach and interpret events (e.g., McCrae et al., 2000), and that personality self-reports remain stable even throughout depressive episodes (Costa, Bagby, Herbst \& McCrae, 2005). Other personality theorists, however, have argued that the expression of traits might be influenced by experience and environmental influences (e.g., Mischel \& Shoda, 1998; Pervin, 1994). Although dispositional mindfulness may precede the appraisal and coping processes that contribute to depressive illness, it remains possible that cumulative exposure to stressors, depressive episodes and coping experiences might reciprocally influence the expression of mindfulness. In an effort to acknowledge and identify potential bi-directional relationships, alternative models were explored. These analyses suggested that the mediated effects were stronger for the proposed model than for alternative models, supporting the contention that trait mindfulness forms a foundation for subsequent appraisals and coping actions that tend to precede the onset of depressive symptoms.

Although not a limitation per se, it should be noted that participant recruitment and data collection for Study 2 was performed online. Given the popularity and widespread accessibility of the internet, combined with the relative anonymity afforded by online participation, it has been argued that this type of reporting might encourage greater rates of random, disingenuous or repeat responding (e.g., participants completing surveys more than once), particularly if a financial incentive is offered for participation.

While these factors were considered when designing Study 2, a large-scale comparison of research modalities found that data collected from online sources did not differ significantly from data collected in more traditional laboratory settings in terms of response validity or participant demographics (Gosling, Vazire, Srivastava \& John, 
2004). Moreover, the results and conclusions drawn from online research were consistent with literature published using more conventional methodology. As an extra safeguard, validity checks were implemented to minimize random responses, and IP addresses were captured to allow for the detection of duplicate survey submissions. Despite the fact that face-to-face interactions are not possible in this type of design and it is difficult to gauge the interest and level of engagement of participants, there is little evidence to suggest that online data are unreliable, and, to our view, this methodology offered several strengths that balanced potential limitations. In particular, this sampling strategy allowed us to reach a broader demographic in terms of age and educational/occupational status, and to compare responses from this broader sample to those obtained from a relatively younger University sample.

In Study 3, we were restricted to offering laboratory sessions in the afternoons in order to control for diurnal changes in cortisol patterns, and were constrained in terms of the length of sessions. From an empirical standpoint, it would have been ideal to schedule participants for longer sessions in the lab in order to collect blood samples at additional time points, and to identify changes in cortisol and cytokine recovery among participants over a longer time horizon; however, these sessions were intensive, involving active preparation and engagement in a stressful activity, as well as continuous blood withdrawal over a 50-minute period. As such, sessions were limited to 90 minutes in order to reduce participant fatigue and encourage participation around student schedules.

Another important limitation to the third study involved high exclusion and attrition rates. In Study 3.1, only $44 \%$ of students who expressed interest and completed a brief screening measure were eligible to participate in the study, and of these, only $63 \%$ 
proceeded to register and complete the laboratory session. In Study 3.2, only $72 \%$ of participants who completed the initial training session returned for the second session and participated in the stressor task and blood collection procedure. Although we do not have a way of knowing why these students did not return for the second session, it is possible that students were deterred from participating due to the length of the individual sessions, design of the research over two separate days, or the invasive nature of the blood collection procedure itself. An analysis of completer vs. non-completer samples indicated that participants who withdrew after the first session did not differ from those who completed the study on key demographic variables (e.g., gender, ethnicity), nor did they differ as a function of Session 1 training condition or pre-existing levels of trait mindfulness or depressive symptoms. It remains possible, however, that these individuals differed on some unmeasured variable (e.g., conscientiousness).

Finally, it should be noted that although a significant rise in cortisol was observed among participants following the Trier Social Stress paradigm in Studies 3.1 and 3.2, this rise of cortisol was lower than anticipated. Previous evaluations of the Trier Social Stress Test (e.g., Kudielka, Hellhammer \& Kirschbaum, 2007) have reported average cortisol increases of two to three times above baseline levels for a majority $(70-80 \%)$ of individuals. In the present samples, baseline cortisol levels were high, and the mean cortisol increase observed across participants was only in the range of $5 \mu \mathrm{g} / \mathrm{dl}$.

Several factors may have contributed to high baseline cortisol levels in the present program of research, including exercise, the consumption of caffeine and/or nicotine prior to testing, or anxiety surrounding the blood sampling procedure. Although participants were pre-screened for anxiety and were asked to refrain from strenuous 
exercise, smoking, eating, and drinking caffeinated or alcoholic beverages for at least one hour prior to the session, we cannot ensure that individuals were fully compliant with these procedures. Additionally, Kudielka et al. (2007) recommended that participants be given a $30-45$ minute rest period to allow hormone levels to stabilize prior to task initiation. Unfortunately, test sessions exceeding 2 hours typically interfere with class time for student participants, and given the number of questionnaires and length of time required to administer the consent forms, stressor task and debriefing, this extended rest period was not feasible in the present investigation. It is thus possible that the time provided to participants in the current sessions did not allow for full acclimatization to the testing environment.

\section{Implications of the Present Research}

Despite advancements in neuroimaging, and behavioural and pharmaceutical treatments for depression (Holtzheimer \& Nemeroff, 2006), it seems that current treatment strategies for depression have achieved only limited success. For instance, in the case of drug therapies, only about $50-60 \%$ of individuals exhibit a positive response, only a partial diminution of symptoms is achieved, and relapse rates approach $70 \%$ over a 5-year period. To make matters worse, rates of depression in North America are continuing to rise, and constitute a major cause of global disease and disability (WHO, 2007). Depression is the greatest antecedent of suicide and a leading cause of death worldwide (Nemeroff, 2008), and has been cited as the fastest growing burden to Canadian employers in terms of absenteeism, diminished workplace productivity, and medical and disability costs (Bender \& Farvolden, 2008; Dewa, Lesage, Goering \& Caveen, 2004). The Mood Disorders Society of Canada estimates that antidepressant use 
has increased in Canada at an average annual rate of $4.6 \%$ since 1998, and that young adults aged $12-20$ years are still among the most vulnerable for developing symptoms (Mood Disorders Society of Canada, 2009; Nemeroff, 2008). Despite increased research attention, depression undeniably continues to represent a serious health concern with farreaching economic, health care and policy implications.

Considering the pervasive, negative impacts of depression at both the individual and societal level, there is a clear need to identify risk and resilience factors for depression, and to evolve more effective and tailored treatments to meet the needs of those affected. The present program of research sought to evaluate the role of mindfulness (both as a trait and as a complement of skills that can be taught and developed) in the onset and progression of depressive illness. Across a series of three studies, trait mindfulness consistently and negatively predicted depressive symptoms, and this relationship was largely driven through indirect pathways involving emotion-focused coping and internalized stigma. Specifically, higher levels of trait mindfulness consistently predicted lower endorsement of emotion-focused coping strategies, and lower endorsement of self-stigma, which in turn uniquely predicted declines in depressive symptoms. When specific facets of trait mindfulness were examined to explore these pathways in greater detail, non-judgment of thoughts and feelings uniquely predicted the observed associations between trait mindfulness, internalized stigma and emotionfocused coping. Taken together, these results highlight the utility of considering the role that specific aspects of trait mindfulness play in the onset and development of depressive illness, and suggest that the practice of mindfulness skills (particularly non-judgment for thoughts and feelings) might confer health benefits by reducing emotion-focused coping 
tendencies and self-stigmatization for depressive symptoms, and promoting the use of problem-oriented and mindful coping efforts in response to stressors.

The present program of research also contributes to the literature on mindfulness and physiological processes implicated in the stressor response. In particular, our results indicate that different facets of trait mindfulness may be instrumental at different stages in the processing of stressful stimuli, with observation and present-moment awareness predicting lower resting/baseline levels of cortisol, and the ability to limit automatic, reflexive responses predicting lower cortisol release when individuals are engaged in stressful tasks.

\section{Conclusion}

The present research extends upon previous literature on mindfulness and mental health to offer additional insight into potential mechanisms of action and pathways underlying this relationship. In particular, the present body of research uniquely contributes to the mindfulness literature by evaluating the role of specific facets of trait mindfulness in the development of internalized stigma, coping, and depressive symptoms among young adults. This research is the first that we know of to directly compare responses on both the MAAS and the FFMQ in this context, and to evaluate the differential role of these variables in predicting well-being within a stress and coping framework.

In conclusion, results of these three studies suggest that trait mindfulness may play a role in mitigating the cortisol response, emotion-focused coping and the internalization of negative stereotypes surrounding depressive symptoms, and that coping and internalized stigma might represent important pathways through which mindfulness 
negatively predicts depressive symptoms. The present research helps to clarify the complex role that trait mindfulness and its underlying facets may play in the relationship between appraisal, stress and mental health processes, and suggests that adopting a facet approach to the study of mindfulness may provide rich insight into the mechanisms through which mindfulness promotes physical and psychological health. An enhanced understanding of these relationships may in turn allow us to better anticipate vulnerability and proactively develop more effective interventions for those at risk of depressive illness. 


\section{References}

Adler, J., Wagner, J., \& McAdams, D. (2007). Personality and the coherence of psychotherapy narratives. Journal of Research in Personality, 41, 1179-1198. doi: 10.1016/j.jrp.2007.02.006.

Aiken, L., \& West, S. (1991). Multiple regression: Testing and interpreting interactions. Newbury Park, CA: Sage.

Akaike, H. (1974). A new look at the statistical model identification. IEEE Transactions on Automatic Control, 19(6), 716-723. doi : 10.1109/TAC.1974.1100705.

Almeida, A., Guindalini, C., Batista-Neves, S., de Oliveira, I., Miranda-Scippa, A., \& Quarantini, L. (2010). Can antidepressants prevent interferon-alpha-induced depression? A review of the literature. General Hospital Psychiatry, 32, 401-405. doi: 10.1016/j.genhosppsych.2010.03.001.

American Psychiatric Association. (1994). Diagnostic and statistical manual of mental disorders (4th ed.). Washington, DC.

Anisman, H. (2009). Cascading effects of stressors and inflammatory immune system activation: Implications for major depressive disorder. Journal of Psychiatry \& Neuroscience, 34(1), 4-20.

Anisman, H., Merali, Z., \& Hayley, S. (2008). Depressive disorders and inflammatory processes. In M. Hersen, \& A. Gross (Eds.), Handbook of clinical psychology (pp. 779-809). Hoboken, NJ: John Wiley \& Sons.

Anisman, H., Ravindran, A.V., Griffiths, J., \& Merali, Z. (1999). Endocrine and cytokine correlates of major depression and dysthymia with typical or atypical features. Molecular Psychiatry, 4, 182-188. 
Antoni, F.A. (1986). Hypothalamic control of adrenocorticotropin secretion: Advances since the discovery of 41-residue corticotropin-releasing factor. Endocrine Reviews, 7(4), 351-378. doi: 10.1210/edrv-7-4-351.

Aspinwall, L., \& Richter, L. (1999). Optimism and self-mastery predict more rapid disengagement from unsolvable tasks in the presence of alternatives. Motivation and Emotion, 23, 221-245. doi: 10.1023/A:1021367331817.

Baer, R., Smith, G., Hopkins, J., Krietemeyer, J., \& Toney, L. (2006). Using self-report assessment methods to explore facets of mindfulness. Assessment, 13(1), 27-45. doi: $10.1177 / 1073191105283504$.

Baer, R., Smith, G., Lykins, E., Button, D., Krietemeyer, J., Sauer, S., et al. (2008). Construct validity of the Five Facet Mindfulness Questionnaire in meditating and nonmeditating samples. Assessment, 15, 329-342. doi: 10.1177/1073191107313003.

Bargh, J. A., Chen, M., \& Burrows, L. (1996). Automaticity of social behavior: Direct effects of trait construct and stereotype activation on action. Journal of Personality and Social Psychology, 71(2), 230-244. doi: 10.1037/00223514.71.2.230.

Barnes, S., Brown, K.W., Krusemark, E., Campbell, W.K., \& Rogge, R. (2007). The role of mindfulness in romantic relationship satisfaction and responses to relationship stress. Journal of Marital and Family Therapy, 33, 482-500. doi: 10.1111/j.17520606.2007.00033. 
Baron, R.M., \& Kenny, D.A. (1986). The moderator-mediator variable distinction in social psychological research: Conceptual, strategic, and statistical considerations. Journal of Personality and Social Psychology, 51, 1173-1182. doi: 10.1037/00223514.51.6.1173.

Beck, A.T. (2008). The evolution of the cognitive model of depression and its neurobiological correlates. American Journal of Psychiatry, 165(8), 969-977. doi: 10.1176/appi.ajp.2008.08050721.

Beck, A. T. (1967). Depression; Clinical, experimental and theoretical aspects. New York, NY: Hoebner Medical Division, Harper \& Row.

Beck, A.T., Rush, A., Shaw, B., \& Emery, G. (1979). Cognitive therapy of depression. New York, NY: Guilford Press.

Beck, A.T., Steer, R., \& Garbin, M. (1988). Psychometric properties of the Beck Depression Inventory: Twenty-five years of evaluation. Clinical Psychology Review, 8, 77-100. doi: 10.1016/0272-7358(88)90050-5.

Beck, A.T., Ward, C., Mendelson, M., Mock, J., \& Erbaugh, J. (1961). An inventory for measuring depression. Archives of General Psychiatry, 4, 561-569.

Beckham, E., Leber, W., Watkins, J., Boyer, J., \& Cook, J. (1986). Development of an instrument to measure Beck's cognitive triad: The cognitive triad inventory. Journal of Consulting and Clinical Psychology, 54, 566-567. doi: 10.1037/0022006X.54.4.566. 
Bender, A., \& Farvolden, P. (2008). Depression and the workplace: A progress report. Current Psychiatry Reports, 10(1), 73-79. doi: 10.1007/s11920-008-0013-6.

Ben-Zeev, D., \& Young, M. (2010). Accuracy of hospitalized depressed patients' and healthy controls' retrospective symptom reports: An experience sampling study. Journal of Nervous and Mental Disease, 198(4), 280-285. doi: 10.1097/NMD.0b013e3181d6141f.

Bishop, S., Lau, M., Shapiro, S., Carlson, L., Anderson, N., Carmody, J., Segal, Z., Abbey, S., Speca, M., Velting, D., \& Devins, G. (2004). Mindfulness: A proposed operational definition. Clinical Psychology: Science and Practice, 11(3), 230-241. doi: 10.1093/clipsy.bph077.

Bränström, R., Kvillemo, P., \& Åkerstedt, T. (2013). Effects of mindfulness training on levels of cortisol in cancer patients. Psychosomatics, 54, 158-164. doi: 10.1016/j.psym.2012.04.007.

Brown, K.W., \& Ryan, R.M. (2003). The benefits of being present: Mindfulness and its role in psychological well-being. Journal of Personality and Social Psychology, 84, 822-848. doi: 10.1037/0022-3514.84.4.822.

Brown, K. W., \& Ryan, R. M. (2004). Perils and promise in defining and measuring mindfulness: Observations from experience. Clinical Psychology: Science and Practice, 11(3), 242-248. doi: 10.1093/clipsy.bph078.

Brown, K.W., Weinstein, N., \& Cresswell, D. (2012). Trait mindfulness modulates neuroendocrine and affective responses to social evaluative threat. Psychoneuroendocrinology, 37, 2037-2041. doi: 10.1016/j.psyneuen.2012.04.003. 
Burnham, K.P., \& Anderson, D.R. (2002). Model selection and multimodel inference: A practical information-theoretic approach ( $2^{\text {nd }}$ ed.). New York, NY: Springer.

Capuron, L., Hauser, P., Hinze-Selch, D., Miller, A.H., \& Neveu, P.J. (2002). Treatment of cytokine-induced depression. Brain, Behavior and Immunity, 16, 575-580. doi: 10.1016/S0889-1591(02)00007-7.

Carmody, J. (2009). Evolving conceptions of mindfulness in clinical settings. Journal of Cognitive Psychotherapy, 23(3), 270-280. doi: 10.1891/0889-8391.23.3.270.

Carver, C., Scheier, M., \& Weintraub, J. (1989). Assessing coping strategies: A theoretically based approach. Journal of Personality and Social Psychology, 56(2), 267-283. doi: 10.1037/0022-3514.56.2.267.

Cash, M., \& Whittingham, K. (2010). What facets of mindfulness contribute to psychological well-being and depressive, anxious, and stress-related symptomatology? Mindfulness, 1, 177-182. doi: 10.1007/s12671-010-0023-4.

Chen, S., Wu, W., \& Bond, M. (2009). Linking family dysfunction to suicidal ideation: Mediating roles of self-views and world-views. Asian Journal of Social Psychology, 12, 133-144. doi: 10.1111/j.1467-839X.2009.01280.x.

Christopher, M., \& Gilbert, B. (2010). Incremental validity of components of mindfulness in the prediction of satisfaction with life and depression. Current Psychology: Research \& Reviews, 29, 10-23. doi: 10.1007/s12144-009-9067-9.

Church, M., \& Brooks, C. (2009). Subtle suicide: Our silent epidemic of ambivalence about living. Santa Barbara, CA: Greenwood. 
Costa, P., Bagby, R., Herbst, J., \& McCrai, R.R. (2005). Personality self-reports are concurrently reliable and valid during acute depressive episodes. Journal of Affective Disorders, 89(1-3), 45-55. doi: 10.1016/j.jad.2005.06.010.

Coyne, J., Gallo, S., Klinkman, M., \& Calarco, M. (1998). Effects of recent and past major depression and distress on self-concept and coping. Journal of Abnormal Psychology, 107(1), 86-96. doi: 10.1037/0021-843X.107.1.86.

Danielson, A.M., Matheson, K., \& Anisman, H. (2011). Cytokine levels at a single time point following a reminder stimulus among women in abusive dating relationships: Relationship to emotional states. Psychoneuroendocrinology, 36(1), 40-50. doi: 10.1016/j.psyneuen.2010.06.003.

Davidson, R., Kabat-Zinn, J., Schumacher, J., Rosenkranz, M., Muller, D., Santorelli, S., Urbanowski, F., Harrington, A., Bonus, K., \& Sheridan, J.F. (2004). Alterations in brain and immune function produced by mindfulness meditation. Psychosomatic Medicine, 65(4), 564-570. doi: 10.1097/01.PSY.0000077505. 67574.E3.

Deikman, A. J. (1982). The observing self. Boston: Beacon Press.

Dewa, C., Lesage, A., Goering, P., \& Caveen, M. (2004). Nature and prevalence of mental illness in the workplace. Healthcare Papers, 5(2), 12-25.

Diener, E., Emmons, R., Larsen, R., \& Griffin, S. (1985). The satisfaction with life scale. Journal of Personality Assessment, 49 (1), 71-75. doi: 10.1207/s15327752jpa4901_13.

Dimeff, L., \& Marlatt, A. (1998). Preventing relapse and maintaining change in addictive behaviors. Clinical Psychology: Science and Practice, 5, 513-525. doi: 10.1111/j.1468-2850.1998.tb00171.x. 
Djikic, M., Langer, E., \& Stapleton, S. (2008). Reducing stereotyping through mindfulness: Effects on automatic, stereotype-activated behaviors. Journal of Adult Development, 15, 106-111. doi: 10.1007/s10804-008-9040-0.

Eisenlorh-Moul, T., Walsh, E., Charnigo, R., Lynam, D., \& Baer, R. (2012). The "What" and the "How" of dispositional mindfulness: Using interactions among subscales of the Five-Facet Mindfulness Questionnaire to understand its relation to substance use. Assessment, 19(3), 276-286. doi: 10.1177/1073191112446658.

Farb, N., Anderson, A., Mayberg, H., Bean, J., McKeon, D., \& Segal, Z. (2010). Minding one's emotions: Mindfulness training alters the neural expression of sadness. Emotion, 10(1), 25-33. doi: 10.1037/a0017151.

Fennell, M. (1997). Low self-esteem: A cognitive perspective. Behavioural and Cognitive Psychotherapy, 25(1), 1-26. doi: 10.1017/S1352465800015368.

Folkman, S., \& Lazarus, R. (1980). An analysis of coping in a middle-aged community sample. Journal of Health and Social Behavior, 21, 219-239. doi: $10.2307 / 2136617$.

Fledderus, M., Bohlmeijer, E.T., \& Pieterse, M.E. (2010). Does experiential avoidance mediate the effects of maladaptive coping styles on psychopathology and mental health? Behavior Modification, 34(6), 503-519. doi: 10.1177/0145445510378379.

Folkman, S., Lazarus, R., Dunkel-Schetter, C., DeLongis, A., \& Gruen, R. (1986). Dynamics of a stressful encounter: Cognitive appraisal, coping, and encounter outcomes. Journal of Personality and Social Psychology, 50, 992-1003. doi: 10.1037/0022-3514.50.5.992. 
Frewen, P., Dozois, D., Neufeld, R., Lane, R., Densmore, M., Stevens, T., \& Lanius, R. (2010). Individual differences in trait mindfulness predict dorsomedial prefrontal and amygdala response during emotional imagery: An fMRI study. Personality and Individual Differences, 49, 479-484. doi: 10.1016/j.paid.2010.05.008.

Gosling, S.D., Vazire, S., Srivastava, S., \& John, O.P. (2004). Should we trust web-based studies? A comparative analysis of six preconceptions about internet questionnaires. American Psychologist, 59(2), 93-104. doi: 10.1037/0003066X.59.2.93.

Grossman, P., Niemann, L., Schmidt, S. \& Walach, H. (2004). Mindfulness-based stress reduction and health benefits: A meta-analysis. Journal of Psychosomatic Research, 57, 35-43. doi: 10.1016/S0022-3999(03)00573-7.

Gully, S. M. (1994, April). Repeated measures regression analyses: A clarification with illustrative examples. In Ninth Annual Conference of the Society for Industrial and Organizational Psychology, Nashville, TN.

Hayes, A. M., \& Feldman, G. (2004). Clarifying the construct of mindfulness in the context of emotion regulation and the process of change in therapy. Clinical Psychology: Science and Practice, 11(3), 255-262. doi: 10.1093/clipsy/bph080.

Heeren, A., \& Philippot, P. (2011). Changes in ruminative thinking mediate the clinical benefits of mindfulness: Preliminary findings. Mindfulness, 2), 8-13. doi: 10.1007/s12671-010-0037-y.

Herwig, U., Kaffenberger, T., Lutz, J., \& Brühl, A. (2010). Self-related awareness and emotion regulation. NeuroImage, 50, 734-741. doi:

10.1016/j.neuroimage.2009.12.089. 
Hick, S., \& Chan, L. (2010). Mindfulness-based cognitive therapy for depression: Effectiveness and limitations. Social Work in Mental Health, 8(3), 225-237. doi: $10.1080 / 15332980903405330$.

Holmes, E.P., \& River, L.P. (1998). Individual strategies for coping with the stigma of severe mental illness. Cognitive and Behavioral Practice, 5, 231-239. doi: $10.1016 / \mathrm{S} 1077-7229(98) 80008-4$.

Holtzheimer, P.E., \& Nemeroff, C.B. (2006). Advances in the treatment of depression. NeuroRx: The Journal of the American Society for Experimental Neuro Therapeutics, 3(1), 42-56. doi: 10.1016/j.nurx.2005.12.007.

Horn, J.L. (1965). A rationale and test for the number of factors in factor analysis. Psychometrika, 30(2), 179-185. doi: 10.1007/BF02289447.

Johnson, K., Johnson, J., \& Petzel, T. (1992). Social anxiety, depression, and distorted cognitions in college students. Journal of Social and Clinical Psychology, 11(2), 181-195. doi: n/a.

Jones, S., \& Day, C. (2008). Self appraisal and behavioural activation in the prediction of hypomanic personality and depressive symptoms. Personality and Individual Differences, 45, 643-648. doi: 10.1016/j.paid.2008.07.008.

Kabat-Zinn, J. (1990). Full catastrophe living: Using the wisdom of your body and mind to face stress, pain, and illness. New York, NY: Delacourt.

Kabat-Zinn, J. (1994). Wherever you go there you are: Mindfulness meditation in everyday life. New York, NY: Hyperion. 
Kato, T. (2012). Development of the coping flexibility scale: Evidence for the coping flexibility hypothesis. Journal of Counseling Psychology, 59(2), 262-273. doi: 10.1037/a0027770.

Kelly, O., Matheson, K., \& Anisman, H. (2003). The Appraisal of Ambiguous Situations Questionnaire. Unpublished manuscript, Carleton University, Ottawa.

Kiecolt-Glaser, J.K., \& Glaser, R. (2002). Depression and immune function: Central pathways to morbidity and mortality. Journal of Psychosomatic Research, 53, 873-876. doi: 10.1016/S0022-3999(02)00309-4.

Kieviet-Stijnen, A., Visser, A., Garssen, B., \& Hudig, W. (2008). Mindfulness-based stress reduction training for oncology patients: Patients' appraisal and changes in well-being. Patient Education and Counseling, 72, 436-442. doi: 10.1016/j.pec.2008.05.015.

Kirschbaum, C., Pirke, K., \& Hellhammer, D. (1993). The 'Trier Social Stress Test': A tool for investigating psychobiological stress responses in a laboratory setting. Neuropsychobiology, 28, 76-81. doi:

Kovacs, M., \& Beck, A.T. (1978). Maladaptive cognitive structures in depression. The American Journal of Psychiatry, 135, 525-533. doi: n/a.

Kudielka, B.M., Helhammer, D., \& Kirschbaum, C. (2007). Ten years of research with the Trier Social Stress Test - revisited. In: E. Harmon-Jones \& P. Winkielman, eds. Social Neuroscience: Integrating Biological and Psychological Explanations of Social Behavior, pp. 56-83. New York, NY: Guilford Press. 
Lamberts, S., Verleun, T., Oosterom, R., DeJong, P., \& Hackeng, W. (1984).

Corticotropin releasing factor and vasopressin exert a synergistic effect on adrenocorticotropin release in man. Journal of Clinical Endocrinology and Metabolism, 58, 298-303. doi: 10.1210/jcem-58-2-298.

Lang, T., Moulds, M., \& Holmes, E. (2009). Reducing depressive intrusions via a computerized cognitive bias modification of appraisals task: Developing a cognitive vaccine. Behaviour Research and Therapy, 47(2), 139-145. doi: 10.1016/j.brat.2008.11.002.

Langer, E. (1989). Mindfulness. Cambridge, MA: Da Capo Press.

Lazarus, R., \& Folkman, S. (1984). Stress, appraisal, and coping. New York, NY: Springer.

Lengacher, C., Kip, K., Barta, M., Post-White, J., Jacobsen, P., Groer, M. et al. (2012). A pilot study evaluating the effect of Mindfulness-Based Stress Reduction on psychological status, physical status, salivary cortisol, and interleukin-6 among advanced-stage cancer patients and their caregivers. Journal of Holistic Nursing, 30(3), 170-185. doi: 10.1177/0898010111435949.

Leung, K., \& Bond, M. H. (2008). Psycho-logic and eco-logic: Insights from social axiom dimensions. In: F. van de Vijver, D. van Hemert \& Y. P. Poortinga, eds. Individuals and Cultures in Multilevel Analysis, pp. 197-219. Mahwah, NJ: Erlbaum. 
Livingston, J.D., \& Boyd, J.E. (2010). Correlates and consequences of internalized stigma for people living with mental illness: A systematic review and metaanalysis. Social Science and Medicine, 71, 2150-2161. doi:

10.1016.j.socscimed.2010.09.030.

Lotrich, F., Rabinovitz, M., Gironda, P., \& Pollock, B. (2007). Depression following pegylated interferon-alpha: Characteristics and vulnerability. Journal of Psychosomatic Research, 63, 131-135. doi: 10.1016/j.jpsychores.2007.05.013.

Lundberg, B., Hansson, L., Wentz, E., \& Bjorkman, T. (2009). Are stigma experiences among persons with mental illness related to perceptions of self-esteem, empowerment and sense of coherence? Journal of Psychiatric and Mental Health Nursing, 16, 516-522. doi: 10.1111/j.1365-2850.2009.01418.x.

Lysaker, P., Roe, D., \& Yanos, P. (2007). Toward understanding the insight paradox: Internalized stigma moderates the association between insight and social functioning, hope, and self-esteem among people with schizophrenia spectrum disorders. Schizophrenia Bulletin, 33(1), 192-199. doi: 10.1093/schbul/sb1016.

Matheson, K, \& Anisman, H. (2003). Systems of coping associated with psychological distress: A profile perspective. Stress, 6, 223-234.

McAdams, D. (1996). Personality, modernity, and the storied self: A contemporary framework for studying persons. Psychological Inquiry, 7, 295-321. doi: 10.1207/s15327965pli0704_1.

McKay, J. (2009). The course of substance use disorders and implications for continuing care. (pp. 19-32). Washington, DC: American Psychological Association. 
McCrae, R.R., Costa, P.T., Ostendorf, F., Angleitner, A., Hrebickova, M., Avia, M.D., Sanz, J., Sanchez-Bernardos, M.L., Kusdil, M.E., Woodfield, R., Saunders, P.R., \& Smith, P.B. (2000). Nature over nurture: Temperament, personality, and lifespan development. Journal of Personality and Social Psychology, 78, 173-186. doi: 10.1037/0022-3514.78.1.173.

Mesters, I. (2009). Motivational interviewing: Hype or hope? Chronic Illness, 5(1), 3-6. doi: $10.1177 / 1742395309102242$.

Miranda, R., \& Nolen-Hoeksema, S. (2007). Brooding and reflection: Rumination predicts suicidal ideation at 1-year follow-up in a community sample. Behaviour Research and Therapy, 45, 3088-3095. doi: 10.1016/j.brat.2007.07.015.

Mischel, W., \& Shoda, Y. (1998). Reconciling processing dynamics and personality dispositions. Annual Review of Psychology, 49, 229-258. doi:

10.1146/annurev.psych.49.1.229.

Mood Disorders Society of Canada (2009). Quick facts: Mental illness and addiction in Canada, $3^{\text {rd }}$ Edition. Retrieved October 2012 from http://www.mooddisorderscanada.ca/

Moses, T. (2009). Stigma and self-concept among adolescents receiving mental health treatment. American Journal of Orthopsychiatry, 79, 261-274. doi: 10.1037/a001596.

Mossner, R., Mikova, O., Koutsilieri, E., Saoud, M., Ehlis, A., Müller, N., et al. (2007). Consensus paper of the WFSBP task force on biological markers: Biological markers in depression. World Journal of Biological Psychiatry, 8, 141-174. 
Neff, K. (2003). The development and validation of a scale to measure self-compassion. Self and Identity, 2, 223-250. doi: 10.1080/15298860390209035.

Neff, K., Rude, S., \& Kirkpatrick, K. (2007). An examination of self-compassion in relation to positive psychological functioning and personality traits. Journal of Research in Personality, 41, 908-916. doi: 10.1016/j.jrp.2006.08.002.

Nemeroff, C.B. (2008). Recent findings in the pathophysiology of depression. FOCUS, 6(1), 3-14. Retrieved from http://focus.psychiatryonline.org.

Niemiec, C., Brown, K.W., Kashdan, T.B., Cozzolino, P.J., Breen, W.E., LevesqueBristol, C., \& Ryan, R.M. (2010). Being present in the face of existential threat: The role of trait mindfulness in reducing defensive responses to mortality salience. Journal of Personality and Social Psychology, 99(2), 344-365. doi: 10.1037/a0019388.

Nolen-Hoeksema, S. (1991). Responses to depression and their effects on the duration of depressive episodes. Journal of Abnormal Psychology, 100(4), 569-582. doi: 10.1037/0021-843X.100.4.569.

Nolen-Hoeksema, S. (2000). The role of rumination in depressive disorders and mixed anxiety/depressive symptoms. Journal of Abnormal Psychology, 109, 504-511. doi: $10.1037 / / 0021-843 X .109 .3 .504$.

Nolen-Hoeksema, S., Wisco, B., \& Lyubomirsky, S. (2008). Rethinking rumination. Perspectives on Psychological Science, 3, 400-424. doi: 10.1111/j.17456924.2008.00088.x. 
Nunes, S., Watanabe, M., Morimoto, H., Moriya, R., \& Reiche, E. (2010). The impact of childhood sexual abuse on activation of immunological and neuroendocrine response. Aggression and Violent Behavior [np]. doi: 10.1016/j.avb.2010.07.006.

Palmer, A., \& Rodger, S. (2009). Mindfulness, stress, and coping among university students. Canadian Journal of Counselling, 43, 3, 198-212. Retrieved from http://cjc-rcc.ucalgary.ca/cjc/index.php/rcc/article/view/648.

Panayiotou, G., \& Papageorgious, M. (2007). Depressed mood: The role of negative thoughts, self-consciousness, and sex role stereotypes. International Journal of Psychology, 42, 289-296. doi: 10.1080/00207590701318389.

Patten, S., Wang, J., Williams, J., Currie, S., Beck, C., Maxwell, C., \& el-Guebaly, N. (2006). Descriptive epidemiology of Major Depression in Canada. The Canadian Journal of Psychiatry, 52(2), 84-90.

Paul, N., Stanton, S.J., Greeson, J.M., Smoski, M.J., \& Wang, L. (2013). Psychological and neural mechanisms of trait mindfulness in reducing depression vulnerability. Social and Cognitive Affective Neuroscience, 8(1), 56-64. doi: $10.1093 / \mathrm{scan} / \mathrm{nss} 070$.

Pervin, L.A. (1994). A critical analysis of current trait theory. Psychological Inquiry, 5(2), 103-113. doi: 10.1207/s15327965pli0502_1.

Preacher, K., Curran, P., \& Bauer, D. (2006). Computational tools for probing interactions in multiple linear regression, multilevel modeling, and latent curve analysis. Journal of Educational and Behavioral Statistics, 31, 437-448. 
Preacher, K. J., \& Hayes, A. F. (2004). SPSS and SAS procedures for estimating indirect effects in simple mediation models. Behavior Research Methods, Instruments, \& Computers, 36(4), 717-731. doi: 10.3758/BF03206553.

Preacher, K. J., \& Hayes, A. F. (2008). Asymptotic and resampling strategies for assessing and comparing indirect effects in multiple mediator models. Behavior Research Methods, 40, 879-891. doi: 10.3758/BRM.40.3.879.

Raison, C., Capuron, L., \& Miller, A. (2006). Cytokines sing the blues: Inflammation and the pathogenesis of major depression. Trends in Immunology, 27(1), 24-31. doi: 10.1016/j.it.2005.11.006.

Rihmer, Z. (2007). Suicide risk in mood disorders. Current Opinion in Psychiatry, 20(1), 17-22. doi: 10.1097/YCO.0b013e3280106868.

Ritsher, J., Otilingam, P., \& Grajales, M. (2003). Internalized stigma of mental illness: Psychometric properties of a new measure. Psychiatry Research, 121(1), 31-49. doi: 10.1016/j.psychres.2003.08.008.

Ritsher, J., \& Phelan, J. (2004). Internalized stigma predicts erosion of morale among psychiatric outpatients. Psychiatry Research, 129(3), 257-265. doi: 10.1016/j.psychres.2004.08.003.

Rood, L., Roelofs, J., Bögels, S., Nolen-Hoeksema, S., \& Schouten, E. (2009). The influence of emotion-focused rumination and distraction on depressive symptoms in non-clinical youth: A meta-analytic review. Clinical Psychology Review, 29, 607-616. doi: 10.1016/j.cpr.2009.07.001. 
Rosenberg, M. (1965). Society and the adolescent self-image. Princeton, NJ: Princeton University Press.

Rosenkranz, M., Davidson, R., MacCoon, D., Sheridan, J., Kalin, N., \& Lutz, A. (2013). A comparison of mindfulness-based stress reduction and an active control in modulation of neurogenic inflammation. Brain, Behavior, and Immunity, 27, 174184. doi: 10.1016/j.bbi.2012.10.013.

Roussi, P., Krikeli, V., Hatzidimitriou, C., \& Koutri, I. (2007). Patterns of coping, flexibility in coping and psychological distress in women diagnosed with breast cancer. Cognitive Therapy and Research, 31, 97-109. doi:10.1007/s10608-006$9110-1$.

Roxburgh, S. (1996). Gender differences in work and well-being: Effects of exposure and vulnerability. Journal of Health and Social Behavior, 37, 265-277. doi: $10.2307 / 2137296$.

Rüsch, N., Corrigan, P., Todd, A., \& Bodenhausen, G. (2010). Implicit self-stigma in people with mental illness. Journal of Nervous and Mental Disease, 198(2), 150153. doi: 10.1097/NMD.0b013e3181cc43b5.

Rüsch, N., Angermeyer, M., \& Corrigan, P. (2005). Mental illness stigma: Concepts, consequences, and initiatives to reduce stigma. European Psychiatry, 20(8), 529539. doi: 10.1016/j.eurpsy.2005.04.004. 
Rüsch, N., Corrigan, P., Wassel, A., Michaels, P., Olschews, M., Wilkniss, S., \& Batia, K. (2009). A stress-coping model of mental illness stigma: Predictors of cognitive stress appraisal. Schizophrenia Research, 110, 59-64. doi:

10.1016/j.schres.2009.01.006.

Ryan, N. (2005). Treatment of depression in children and adolescents. The Lancet, 366 : 9489, 933-940.

Ryff, C. (1989). Happiness is everything, or is it? Explorations on the meaning of psychological well-being. Journal of Personality and Social Psychology, 57, 1069-1081. doi: 10.1037/0022-3514.57.6.1069.

Sanders, W., \& Lam, D. (2010). Ruminative and mindful self-focused processing modes and their impact on problem solving in dysphoric individuals. Behaviour Research and Therapy [in press]. doi: 10.1016/j.brat.2010.04.007.

Sapolsky, R., Romero, M., \& Munck, A. (2000). How do glucocorticoids influence stress responses? Integrating permissive, suppressive, stimulatory, and preparative actions. Endocrine Reviews, 21(1), 55-89. doi: n/a.

Scheier, M., Carver, C., \& Bridges, M. (1994). Distinguishing optimism from neuroticism (and trait anxiety, self-mastery, and self-esteem): A reevaluation of the Life Orientation Test. Journal of Personality and Social Psychology, 67, 1063-1078. doi: 10.1037/0022-3514.67.6.1063. 
Segal, Z.V., Williams, J.M., \& Teasdale, J.D. (2002). Mindfulness-based cognitive therapy for depression: A new approach to preventing relapse. New York, NY: Guilford Press.

Segal, Z.V., Williams, J.M., Teasdale, J.D, \& Gemar, M. (1996). A cognitive science perspective on kindling and episode sensitization in recurrent affective disorder. Psychological Medicine, 26, 371-380. doi: 10.1017/S0033291700034760.

Seiffge-Krenke, I., \& Klessinger, N. (2000). Long-term effects of avoidant coping on adolescents' depressive symptoms. Journal of Youth and Adolescence, 29(6), 617630. doi: 10.1023/A:1026440304695.

Sher, L., Oquendo, M., \& Mann, J. (2001). Risk of suicide in mood disorders. Clinical Neuroscience Research, 1, 337-344. doi: 10.1016/S1566-2772(01)00035-4.

Smith, H., \& Betz, N. (2002). An examination of efficacy and esteem pathways to depression in young adulthood. Journal of Counseling Psychology, 49, 438-448. doi: 10.1037//0022-0167.49.4.438.

Spielberger, C., Gorsuch, R., Lushene, R., Vagg, P., \& Jacobs, G. (1983). Manual for the State-Trait Anxiety Inventory. Palo Alto, CA: Consulting Psychologists Press.

Steer, R., Ball, R., Ranieri, W., \& Beck, A.T. (1999). Dimensions of the Beck Depression Inventory-II in clinically depressed outpatients. Journal of Clinical Psychology, 55, 117-128. doi: 10.1002/(SICI)1097-4679(199901)55:1.

Stein, K., \& Markus, H. (1996). The role of the self in behavioural change. Journal of Psychotherapy Integration, 6(4), 349-384. doi: n/a. 
Suls, J., \& Fletcher, B. (1985). The relative efficacy of avoidant and nonavoidant coping strategies: A meta-analysis. Health Psychology, 4(3), 249-288. doi: 10.1037/0278-6133.4.3.249.

Tabachnick, B., \& Fidell, L. (2007). Using multivariate statistics ( $5^{\text {th }}$ ed.). Boston, MA: Pearson.

Tart, C. (1994). Living the mindful life. Boston, MA: Shambhala.

Taylor, S., \& Aspinwall, L. (1996). Mediating and moderating processes in psychosocial stress: Appraisal, coping, resistance and vulnerability. In H. B. Kaplan (Ed.), Psychosocial stress: Perspectives on structure, theory, life-course, and methods (pp. 71-110). Orlando, FL: Academic Press.

Taylor, V., Grant, J., Daneault, V., Scavone, G., Breton, E., Roffe-Vidal, S., Courtemanche, J., Lavarenne, A., \& Beauregard, M. (2011). Impact of mindfulness on the neural responses to emotional pictures in experienced and beginner meditators. Neuroimage, 57, 1524-1533. doi:

10.1016/j.neuroimage.2011.06.001.

Teasdale, J. (1983). Negative thinking in depression: Cause, effect, or reciprocal relationship. Advances in Behaviour Research and Therapy, 5(1), 3-25. doi: 10.1016/0146-6402(83)90013-9.

Teasdale, J., Segal, Z., \& Williams, J.M. (1995). How does cognitive therapy prevent depressive relapse and why should attentional control (mindfulness) training help? Behaviour Research and Therapy, 33(1), 25-39. doi: 10.1016/00057967(94)E0011-7. 
Teasdale, J., Segal, Z., Williams, J.M., Ridgeway, V., Soulsby, J., \& Lau, M. (2000). Prevention of relapse/recurrence in Major Depression by mindfulness-based cognitive therapy. Journal of Consulting and Clinical Psychology, 68, 615-623. doi: 10.1037//0022-006X.68.4.615.

Van Dam, N., Earleywine, M., \& Borders, A. (2010). Measuring mindfulness? An item response theory analysis of the Mindful Attention Awareness Scale. Personality and Individual Differences, 49(7), 805-810. doi: 10.1016/j.paid.2010.07.020.

Vieten, C., \& Astin, J. (2008). Effects of a mindfulness-based intervention during pregnancy on prenatal stress and mood: Results of a pilot study. Archives of Women's Mental Health, 11(1), 67-74. doi: 10.1007/s00737-008-0214-3.

Wang, J., Williams, J., Lavorato, D., Schmitz, N., Dewa, C., \& Patten, S. (2009). The incidence of Major Depression in Canada: The national population health survey. Journal of Affective Disorders, [np]. doi: 10.1016/j.jad.2009.07.016.

Wann, B.P., Audet, M-C., \& Anisman, H. (2010). Impact of acute and chronic stressor experiences on heart atrial and brain natriuretic peptides in response to a subsequent stressor. Hormones \& Behavior [np]. doi: 10.1016/j.yhbeh.2010.09.001.

Watkins, E., \& Moulds, M. (2005). Distinct modes of ruminative self-focus: Impact of abstract versus concrete rumination on problem solving in depression. Emotion, 5, 319-328. doi: 10.1037/1528-3542.5.3.319.

Watson, D., Clark, L., \& Tellegen, A. (1988). Development and validation of brief measures of positive and negative affect: The PANAS scales. Journal of Personality and Social Psychology, 54, 1063-1070. doi: 10.1037/00223514.54.6.1063. 
Weinstein, N., Brown, K., \& Ryan, R. (2009). A multi-method examination of the effects of mindfulness on stress attribution, coping, and emotional well-being. Journal of Research in Personality, 43(3), 374-385. doi:

10.1016/j.jrp.2008.12.008.

Weissman, M. M., Wolk, S., Wickramaratnre, P., Goldstein, R. B., Adams, P., \& Greenwald, S. (1999). Children with prepubertal-onset major depressive disorder and anxiety grown up. Archives of General Psychiatry, 56, 794-801. doi: 10.1001/archpsyc.56.9.794.

Wheaton, B. (1990). Life transitions, role histories, and mental health. American Sociological Review, 55, 209-223. doi: 10.2307/2095627.

Williams, J.M. (2008). Mindfulness, depression and modes of mind. Cognitive Therapy Research, 32, 721-733. doi: 10.1007/s10608-008-9204-z.

Williams, J.M., \& Swales, M. (2004). The use of mindfulness-based approaches for suicidal patients. Archives of Suicide Research, 8(4), 315-329. doi: $10.1080 / 13811110490476671$.

Wilson, E.B., \& Hilferty, M.M. (1931). The distributions of chi-square. Proceedings of the National Academy of Sciences, 17(12), 684-688.

Witek-Janusek, L., Albuquerque, K., Rambo Chroniak, K., Chroniak, C., Durazo-Arvizu, R., \& Mathews, H. (2008). Effect of mindfulness based stress reduction on immune function, quality of life and coping in women newly diagnosed with early stage breast cancer. Brain, Behavior and Immunity, 22, 969-981. doi: 10.1016/j.bbi.2008.01.012. 
World Health Organization. (2007). Depression. Retrieved November 22, 2009, from http://www.who.int/mental_health/management/depression/definition/en/

Zeidan, F., Johnson, S.K., Gordon, N.S., \& Goolkasian, P. (2010). Effects of brief and sham mindfulness meditation on mood and cardiovascular variables. Journal of Alternative and Complementary Medicine, 16, 867-873. doi:

10.1089/acm.2009.0321. 


\section{Appendix A: Study 1 Recruitment Notice}

\section{Electronic Sign-up System Information}

Study Name: Self-Appraisals, Coping, and Well-Being

Brief Abstract: This study examines how self-appraisals influence people's appraisals of different situations, and how these appraisals influence coping strategies and well-being.

Detailed Description: We are interested in exploring the relationship between personal characteristics (e.g., demographics, depressive symptoms, mindfulness), how individuals appraise a) themselves, and b) different types of situations, and the types of strategies individuals use for coping with stressful situations. We are further interested in examining how these variables are related to well-being. The session requires completion of a questionnaire package regarding personal characteristics, appraisals, the use of various types of coping strategies, and well-being. We will also ask you to write a short description of a stressful event you have recently experienced.

Duration: 1.5 hours

Credits: $1.5 \%$ increase 


\section{Appendix B: Study 1 Informed Consent}

The purpose of an informed consent is to insure that you understand the purpose of the study and the nature of your involvement. The informed consent must provide sufficient information such that you have the opportunity to determine whether you wish to participate in the study.

\section{Present study: Self-Appraisals, Coping, and Well-Being}

Research personnel. The following people are involved in this research project, and may be contacted at any time if you have questions or concerns: Kelly-Lyn Christie (email: klchrist@connect.carleton.ca, phone: 613-520-2600, ext. 6312), Valerie Repta (email: vrepta@connect.carleton.ca), Dr. Hymie Anisman (Faculty Sponsor, email: hanisman@connect.carleton.ca),or Dr. Kimberly Matheson (email: kim_matheson@carleton.ca). Should you have any ethical concerns about this research, please contact Dr. Monique Sénéchal, at :monique_senechal@carleton.ca (613-520-2600 ext.1155).

Purpose. The purpose of this study is to investigate the relationship between personal characteristics (e.g., demographics, depressive symptoms, mindfulness) and how individuals appraise themselves and different types of situations. We are interested in examining how these personal characteristics and appraisals influence the types of strategies people use when dealing with stressful situations, as well as how these variables predict well-being.

Task requirements. We are asking you to fill out a number of questionnaires regarding your background (e.g., demographics), personal characteristics (e.g., how you cope with things in your life), and how you feel about yourself (e.g., self-appraisals, well-being). We will also ask you to write a brief description of a stressful event you have experienced. The questionnaires will take about 90 minutes to complete. You will receive a $1.5 \%$ increase in your final grade of PSYC 1001, PSYC 1002, PSYC 2001 or PSYC 2002 for participating in this study. Testing will take place at Carleton University.

Potential risk/discomfort. There are no physical risks to participating in this study. Some individuals might feel uncomfortable when asked to recall stressful situations or to answer personal, sensitive questions about the coping strategies they use or feelings of depression they may have experienced. If you feel any discomfort or distress, you may choose not to answer specific questions, and you will not be penalized in any way if you do this. The debriefing form at the end of the study provides contact information for local support services that you may contact if you need or want help.

Anonymity/Confidentiality. The data collected in this experiment are strictly confidential. All data are coded such that your name is not associated with the responses you provide. Any identifying information associated with your code will be confined to a single page that will be separated from your questionnaire, and kept in a separate, secured file by the research investigators, who will keep this information confidential.

Right to withdraw. Your participation in this study is entirely voluntary. At any point during the study, you have the right to not complete certain questions, or to withdraw from the study without academic penalty. 


\section{Signatures}

I have read the above form and understand the conditions of my participation. My participation in this study is voluntary, and I understand that if at any time I wish to leave the experiment, I may do so without having to give an explanation and with no penalty whatsoever. Furthermore, I am also aware that the data gathered in this study are confidential and anonymous with respect to my personal identity. My signature indicates that I agree to participate in this study.

Full Name (please print):

Participant Signature:

Date:

Researcher Signature:

Date: 


\section{Appendix C: Study 1 Questionnaires}

\section{Demographic Questionnaire}

Please fill out the following information about yourself and your background:

1. Age:

2. Gender (circle one): Male / Female

3. Marital Status (circle one):

Never Married / Married / Separated / Divorced / Widowed / Common-Law (living together)
1
2
3
4
5 6

4. Ethnicity (select from a-h below):
a) Caucasian/European origin
b) African-Canadian/American
c) East Asian (Chinese, Japanese, Korean)
d) South Asian (Indian, Pakistani, Sri Lankan, etc.)
e) Middle Eastern
f) Native Canadian/American
g) Hispanic and South American Origin
h) Other or multi-ethnic origin

5. Are you currently a student? (Circle one)

YES NO 
a) If YES, what is your student status?

1. Full-time

2. Part-time

3. Special student

b) If YES, what is your major?

c) If YES, what is your current GPA?

6. What is the highest level of education you have completed? (please select one):
a) Elementary School
b) High School
c) College
d) University (undergraduate)
e) University (graduate)

7. Have you ever experienced depression or anxiety? (circle one) YES NO

a) If YES, have you ever sought treatment (e.g., counselling) for these symptoms? YES NO

b) Are you currently being treated for depression or anxiety? YES NO 


\section{$\underline{\text { RSES }}$}

Please indicate your degree of agreement or disagreement with each of the following statements by circling the appropriate option for each statement.

\begin{tabular}{ccccccc}
-3 & -2 & -1 & 0 & 1 & 2 & 3 \\
Strongly & Moderately & Mildly & Neither true & Mildly & Moderately & Strongly \\
untrue & untrue & untrue & nor untrue & true & true & true \\
& & & & & & \\
\hline
\end{tabular}

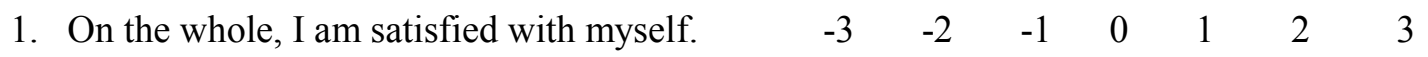

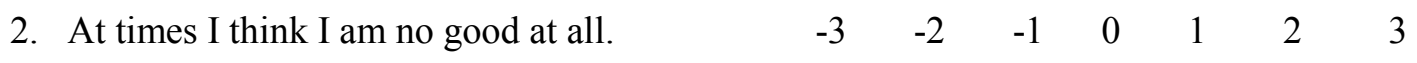

3. I feel that I have a number of good qualities. $\quad \begin{array}{lllllllll}-3 & -2 & -1 & 0 & 1 & 2 & 3\end{array}$

4. I am able to do things as well as most other

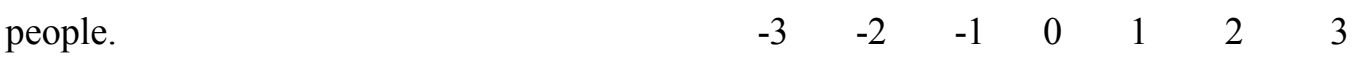

5. I feel I do not have much to be proud of. $\quad \begin{array}{lllllllll}-3 & -2 & -1 & 0 & 1 & 2 & 3\end{array}$

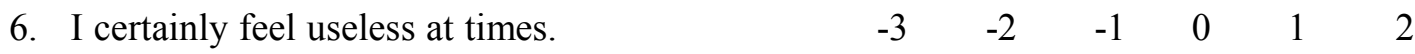
3

7. I feel that I'm a person of worth, at least on an $\begin{array}{llllllll}\text { equal plane with others. } & -3 & -2 & -1 & 0 & 1 & 2 & 3\end{array}$

8. I wish I could have more respect for $\begin{array}{llllllll}\text { myself. } & -3 & -2 & -1 & 0 & 1 & 2 & 3\end{array}$

9. All in all, I am inclined to feel that I am a failure.

$\begin{array}{lllllll}-3 & -2 & -1 & 0 & 1 & 2 & 3\end{array}$

10. I take a positive attitude toward myself. $\quad \begin{array}{lllllllll}-3 & -2 & -1 & 0 & 1 & 2 & 3\end{array}$ 


\section{$\underline{\text { LOT-R }}$}

For each of the following statements, please indicate the extent to which the statement applies to you. Please respond as you really feel, rather than how you think 'most people' feel. Use the rating scale indicated.

Never Seldom Sometimes Often Almost

always

1. In uncertain times, I usually expect the best.

0

1

2

3

4

2. It's easy for me to relax .

0

1

2

3

4

3. If something can go wrong for me, it will.

0

1

2

3

4

4. I'm always optimistic about my future.

0

1

2

3

4

5. I enjoy my friends a lot.

0

1

2

34

6. It's important for me to keep busy.

0

1

2

34

7. I hardly ever expect things to go my way.

0

1

2

3

4

8. I don't get upset too easily.

0

1

2

34

9. I rarely count on good things happening to me. to me than bad. 
RWB - A

\begin{tabular}{|c|c|c|c|c|c|}
\hline 1 & $\underline{2}$ & $\underline{3}$ & $\underline{4}$ & $\underline{5}$ & $\underline{6}$ \\
\hline Strongly & Moderately & Slightly & Slightly & Moderately & Strongly \\
\hline
\end{tabular}

1. Sometimes I change the way I act or think to be more like those around me.

$\begin{array}{llllll}1 & 2 & 3 & 4 & 5 & 6\end{array}$

2. I am not afraid to voice my opinions, even when they are in opposition to the opinions of most people.

$\begin{array}{llllll}1 & 2 & 3 & 4 & 5 & 6\end{array}$

3. My decisions are not usually influenced by what everyone else is doing.
1
2
3
4
5
6

4. I tend to worry about what other people think of me.
1
2
3
4
5
6

5. Being happy with myself is more important to me than having others approve of me.
12
3
$4 \quad 5$
6

6. I tend to be influenced by people with strong opinions.

$\begin{array}{lllllll}1 & 2 & 3 & 4 & 5 & 6\end{array}$

7. People rarely talk me into doing things I don't want to do.

$\begin{array}{llllll}1 & 2 & 3 & 4 & 5 & 6\end{array}$

8. It is more important to me to "fit in" with others than to stand alone on my principles.

$\begin{array}{llllll}1 & 2 & 3 & 4 & 5 & 6\end{array}$

9. I have confidence in my opinions, even if they are contrary to the general consensus.

$\begin{array}{llllll}1 & 2 & 3 & 4 & 5 & 6\end{array}$ 
10. It's difficult for me to voice my own opinions on controversial matters.

$\begin{array}{llllll}1 & 2 & 3 & 4 & 5 & 6\end{array}$

11. I often change my mind about decisions if my friends or family disagree.

$\begin{array}{lllllll}1 & 2 & 3 & 4 & 5 & 6\end{array}$

12. I am not the kind of person who gives in to social pressures to think or act in certain ways.

$\begin{array}{llllll}1 & 2 & 3 & 4 & 5 & 6\end{array}$

13. I am concerned about how other people evaluate the choices I have made in my life.

$\begin{array}{lllllll}1 & 2 & 3 & 4 & 5 & 6\end{array}$

14. I judge myself by what I think is important, not by the values of what others think is important.

$\begin{array}{llllll}1 & 2 & 3 & 4 & 5 & 6\end{array}$ 
RWB - M

\begin{tabular}{|c|c|c|c|c|c|}
\hline$\frac{\frac{1}{\text { Strongly }}}{\text { disagree }}$ & $\frac{\underline{2}}{\text { Moderately }}$ & $\frac{\frac{3}{\text { Slightly }}}{\text { disagree }}$ & $\frac{\frac{4}{\text { Slightly }}}{\text { agree }}$ & $\frac{\frac{5}{\text { Moderately }}}{\text { agree }}$ & $\frac{\underline{6}}{\text { Strongly }}$ \\
\hline
\end{tabular}

1. In general, I feel I am in charge of the situation in which I live.

$\begin{array}{llllll}1 & 2 & 3 & 4 & 5 & 6\end{array}$

2. The demands of everyday life often get me down.

$\begin{array}{llllll}1 & 2 & 3 & 4 & 5 & 6\end{array}$

3. I do not fit very well with the people and the community around me.

$\begin{array}{llllll}1 & 2 & 3 & 4 & 5 & 6\end{array}$

4. I am quite good at managing the many responsibilities of my daily life.

$\begin{array}{llllll}1 & 2 & 3 & 4 & 5 & 6\end{array}$

5. I often feel overwhelmed by my responsibilities.

$\begin{array}{llllll}1 & 2 & 3 & 4 & 5 & 6\end{array}$

6. If I were unhappy with my living situation, I would take effective steps to change it.

$\begin{array}{llllll}1 & 2 & 3 & 4 & 5 & 6\end{array}$

7. I generally do a good job of taking care of my personal finances and affairs.
1
23
$4 \quad 5$
6

8. I find it stressful that I can't keep up with all of the things I have to do each day.
12
3
4
5
6

9. I am good at juggling my time so that I can fit everything in that needs to get done.

$\begin{array}{llllll}1 & 2 & 3 & 4 & 5 & 6\end{array}$


10. My daily life is busy, but I derive a sense of satisfaction from keeping up with everything.

$\begin{array}{llllll}1 & 2 & 3 & 4 & 5 & 6\end{array}$

11. I get frustrated when trying to plan my daily activities because I never accomplish the things I set out to do.

$\begin{array}{llllll}1 & 2 & 3 & 4 & 5 & 6\end{array}$

12. My efforts to find the kind of activities and relationships that I need have been quite unsuccessful.

$\begin{array}{llllll}1 & 2 & 3 & 4 & 5 & 6\end{array}$

13. I have difficulty arranging my life in a way that is satisfying to me.

$\begin{array}{llllll}1 & 2 & 3 & 4 & 5 & 6\end{array}$

14. I have been able to build a home and a lifestyle that is much to my liking.

$\begin{array}{llllll}1 & 2 & 3 & 4 & 5 & 6\end{array}$




\section{$\underline{\text { BDI }}$}

On this questionnaire are groups of statements. Please read the entire group of statements in each category. Then pick out ONE statement in that group which best describes the way you feel. Check off the number beside the statement you have chosen.

1. _ $0 \quad=$ I do not feel sad

$1=$ I feel sad or blue

$2 \mathrm{a}=\mathrm{I}$ am blue or sad all of the time and I can't snap out of it

$2 \mathrm{~b}=\mathrm{I}$ am so sad or unhappy that it is very painful

3 = I am so sad or unhappy that I can't stand it

2. 0 _ 0 I am not particularly pessimistic or discouraged about the future

$1=$ I feel discouraged about the future

$2 \mathrm{a}=\mathrm{I}$ feel I have nothing to look forward to

$2 \mathrm{~b}=\mathrm{I}$ feel I won't every get over my troubles

$3=$ I feel that the future is hopeless and things cannot improve

3. 0 _ $\quad$ I do not feel like a failure

$1=\mathrm{I}$ feel I have failed more than the average person

$2 \mathrm{a}=\mathrm{I}$ feel I have accomplished very little that is worthwhile or that means anything

$2 \mathrm{~b}=$ As I look back on my life, all I can see is a lot of failures

$3=\mathrm{I}$ feel I am a complete failure as a person

4. $\quad 0=$ I am not particularly dissatisfied

$1 \mathrm{a}=\mathrm{I}$ feel bored most of the time

$1 b=I$ don't enjoy things the way I used to

2 = I don't get satisfaction out of anything anymore

3 = I am dissatisfied with everything 
5. __ 0 = I don't feel particularly guilty

$1=$ I feel bad or unworthy a good part of the time

$2 \mathrm{a}=\mathrm{I}$ feel quite guilty

$2 b=I$ feel bad or unworthy practically of the time now

$3=$ I feel as though I am very bad or worthless

6. $\quad 0=$ I don't feel I am being punished

1 = I have a feeling that something bad may happen to me

$2=$ I feel I am being punished or will be punished

$3 \mathrm{a}=\mathrm{I}$ feel I deserve to be punished

$3 \mathrm{~b}=\mathrm{I}$ want to be punished

7. _ 0 _ 0 I don't feel disappointed in myself

$\ldots$

$1 \mathrm{~b}=\mathrm{I}$ don't like myself

$2=$ I am disgusted with myself

$3=$ I hate myself

8. _ $0=$ I do not feel I am any worse than anybody else

$1=$ I am very critical of myself for my weaknesses or mistakes

$2 \mathrm{a}=$ I blame myself for everything that goes wrong

$2 \mathrm{~b}=\mathrm{I}$ feel I have many bad faults 
9. _ 0 _ $\quad$ I don't have thoughts of harming myself

1 = I have thoughts of harming myself but I would not carry them out

$2 \mathrm{a}=\mathrm{I}$ feel I would be better off dead

$2 \mathrm{~b}=\mathrm{I}$ have definite plans about committing suicide

$2 \mathrm{c}=\mathrm{I}$ feel my family would be better off if I were dead

3 = I would kill myself if I could

10. $0=$ I don't cry any more than usual

$1=$ I cry more now than I used to

$2=$ I cry all the time now. I can't stop it.

3 = I used to be able to cry but now I can't cry at all even though I want to

11. $0=\mathrm{I}$ am no more irritated now than I ever am

$1=$ I get annoyed or irritated more easily than I used to

$2=$ I get irritated all the time

3 = I don't get irritated at all the things that used to irritate me.

12. $0=$ I have not lost interest in other people

$1=\mathrm{I}$ am less interested in other people than I used to be

2 = I have lost most of my interest in other people and I have little feeling for them

3 = I have lost all my interest in other people and don't care about them at all

13. $0=$ I make decisions about as well as ever

$1=\mathrm{I}$ am less sure of myself now and try to put off making decisions

$2=$ I can't make decisions anymore without help

3 = I can't make decisions at all anymore 
14. 0 _ 0 I don't feel I look any worse than I used to

$1=\mathrm{I}$ am worried that I am looking old or unattractive

$2=\mathrm{I}$ feel that there are permanent changes in my appearance and they make me look unattractive

$3=$ I feel that I am ugly or repulsive looking

15. 0 _ 0 I can work about as well as before

$1 \mathrm{a}=$ It takes extra effort to get started at doing something $1 \mathrm{~b}=\mathrm{I}$ don't work as well as I used to

2 = I have to push myself very hard to do anything

$3=$ I can't do any work at all

16. _ $0=$ I can sleep as well as usual

$1=$ I wake up more tired in the morning than I used to

2 = I wake up 1-2 hours earlier than usual and find it hard to get back to sleep

3 = I wake up early every day and can't get more than 5 hours sleep

17. 0 _ 0 I don't get any more tired than usual

$1=$ I get tired more easily than I used to

$2=$ I get tired from doing anything

$3=\mathrm{I}$ get too tired to do anything

18. $0=$ My appetite is no worse than usual

$1=$ My appetite is not as good as it used to be

$2=$ My appetite is much worse now

3 = I have no appetite at all any more 
19. _ $0=$ I haven't lost much weight, if any, lately

$1=\mathrm{I}$ have lost more than 5 pounds

$2=$ I have lost more than 10 pounds

3 = I have lost more than 15 pounds

20. __ $0=$ I am no more concerned about my health than usual

$1=\mathrm{I}$ am concerned about aches and pains or upset stomach or constipation or other unpleasant feelings in my body

$2=\mathrm{I}$ am so concerned with how I feel or what I feel that it's hard to think of much else

3 = I am completely absorbed in what I feel

21. $0=$ I have not noticed any recent change in my interest in sex

$1=\mathrm{I}$ am less interested in sex than I used to be

$2=\mathrm{I}$ am much less interested in sex now

3 = I have lost interest in sex completely 


\section{$\underline{\text { ISMIS }}$}

Instructions: Many people feel down or depressed from time to time. Please think of times when you have felt this way, and circle the response that best describes how much you agree or disagree with each statement. There are no right or wrong answers. Even if you are not currently feeling depressed, please respond according to how you would typically feel when you are down.

1. When I'm feeling down, I don't socialize as much as I usually do.

$\begin{array}{ccccc}\text { Strongly Disagree } & \text { Disagree } & \text { Neither Agree nor Disagree } & \text { Agree } & \begin{array}{r}\text { Completely } \\ \text { Agree }\end{array} \\ 0 & 1 & 2 & 3 & 4\end{array}$

2. When I feel down, I sometimes feel inferior to others.

$\begin{array}{ccccc}\text { Strongly Disagree } & \text { Disagree } & \text { Neither Agree nor Disagree } & \text { Agree } & \begin{array}{r}\text { Completely } \\ \text { Agree }\end{array} \\ 0 & 1 & 2 & 3 & 4\end{array}$

3. I avoid getting close to people who don't understand how I feel.

$\begin{array}{ccccc}\text { Strongly Disagree } & \text { Disagree } & \text { Neither Agree nor Disagree } & \text { Agree } & \begin{array}{r}\text { Completely } \\ \text { Agree }\end{array} \\ 0 & 1 & 2 & 3 & 4\end{array}$

4. People could not possibly understand how I feel when I'm depressed.

$\begin{array}{ccccc}\text { Strongly Disagree } & \text { Disagree } & \text { Neither Agree nor Disagree } & \text { Agree } & \begin{array}{r}\text { Completely } \\ \text { Agree }\end{array} \\ 0 & 1 & 2 & 3 & 4\end{array}$

5. I am embarrassed or ashamed of feeling down.

$\begin{array}{ccccc}\text { Strongly Disagree } & \text { Disagree } & \text { Neither Agree nor Disagree } & \text { Agree } & \begin{array}{r}\text { Completely } \\ \text { Agree }\end{array} \\ 0 & 1 & 2 & 3 & 4\end{array}$


6. I stay away from social situations when I'm feeling down.

$\begin{array}{ccccc}\text { Strongly Disagree } & \text { Disagree } & \text { Neither Agree nor Disagree } & \text { Agree } & \begin{array}{r}\text { Completely } \\ \text { Agree }\end{array} \\ 0 & 1 & 2 & 3 & 4\end{array}$

7. People can usually tell when I'm feeling depressed.

$\begin{array}{ccccc}\text { Strongly Disagree } & \text { Disagree } & \text { Neither Agree nor Disagree } & \text { Agree } & \begin{array}{r}\text { Completely } \\ \text { Agree }\end{array} \\ 0 & 1 & 2 & 3 & 4\end{array}$

8. When I'm feeling down, I feel out of place in the world.

$\begin{array}{ccccc}\text { Strongly Disagree } & \text { Disagree } & \text { Neither Agree nor Disagree } & \text { Agree } & \begin{array}{r}\text { Completely } \\ \text { Agree }\end{array} \\ 0 & 1 & 2 & 3 & 4\end{array}$

9. People often patronize me for feeling down.

$\begin{array}{ccccc}\text { Strongly Disagree } & \text { Disagree } & \text { Neither Agree nor Disagree } & \text { Agree } & \begin{array}{r}\text { Completely } \\ \text { Agree }\end{array} \\ 0 & 1 & 2 & 3 & 4\end{array}$

10. People tend to ignore me or take me less seriously when I'm feeling down or depressed.

$\begin{array}{ccccc}\text { Strongly Disagree } & \text { Disagree } & \text { Neither Agree nor Disagree } & \text { Agree } & \begin{array}{r}\text { Completely } \\ \text { Agree }\end{array} \\ 0 & 1 & 2 & 3 & 4\end{array}$

11. I am disappointed in myself for feeling the way I do.

$\begin{array}{ccccc}\text { Strongly Disagree } & \text { Disagree } & \text { Neither Agree nor Disagree } & \text { Agree } & \begin{array}{r}\text { Completely } \\ \text { Agree }\end{array} \\ 0 & 1 & 2 & 3 & 4\end{array}$


12. Feeling depressed or down has spoiled my life.

$\begin{array}{ccccc}\text { Strongly Disagree } & \text { Disagree } & \text { Neither Agree nor Disagree } & \text { Agree } & \begin{array}{r}\text { Completely } \\ \text { Agree }\end{array} \\ 0 & 1 & 2 & 3 & 4\end{array}$

13. When I feel down, I can't contribute anything to society.

$\begin{array}{ccccc}\text { Strongly Disagree } & \text { Disagree } & \text { Neither Agree nor Disagree } & \text { Agree } & \begin{array}{r}\text { Completely } \\ \text { Agree }\end{array} \\ 0 & 1 & 2 & 3 & 4\end{array}$

14. When I feel down, I don't think people are interested in getting close to me.

$\begin{array}{ccccc}\text { Strongly Disagree } & \text { Disagree } & \text { Neither Agree nor Disagree } & \text { Agree } & \begin{array}{r}\text { Completely } \\ \text { Agree }\end{array} \\ 0 & 1 & 2 & 3 & 4\end{array}$




\section{SCOPE}

The purpose of this questionnaire is to find out how people deal with their problems or the stresses in their lives. The following are activities that you may have done. After each activity, please indicate the extent to which you have used this as a way of dealing with problems or stresses in recent weeks.

Ordinarily, in recent weeks, I have:

1. accepted that there is nothing I could do to change my situation.

2. blamed myself for my problems.

3. told others that I was really upset.

4. asked others for help or advice.

5. spent a lot of time thinking about my problems.

6. taken time for recreation or pleasure activities.

7. made plans to overcome my concerns or problems.

8. avoided thinking about my problems.

9. told jokes about my situation.

10. thought a lot about who was responsible for my problems (besides me).

11. worried about my problems a lot.

12. made humorous comments or stories about my situation.

13. wished the situation would just go away or be over with

14. thought a lot about how I brought

my problems on myself.

15. decided to wait and see how things turned out.
Not at all 0

2 3

4

Somewhat

Totally

$\begin{array}{llllll}0 & 1 & 2 & 3 & 4\end{array}$

$\begin{array}{lllll}0 & 1 & 2 & 3 & 4\end{array}$

$\begin{array}{llllll}0 & 1 & 2 & 3 & 4\end{array}$

$\begin{array}{llllll}0 & 1 & 2 & 3 & 4\end{array}$

$\begin{array}{llllll}0 & 1 & 2 & 3 & 4\end{array}$

$\begin{array}{lllll}0 & 1 & 2 & 3 & 4\end{array}$

$\begin{array}{lllll}0 & 1 & 2 & 3 & 4\end{array}$

$\begin{array}{lllll}0 & 1 & 2 & 3 & 4\end{array}$

$\begin{array}{lllll}0 & 1 & 2 & 3 & 4\end{array}$

$\begin{array}{lllll}0 & 1 & 2 & 3 & 4\end{array}$

$\begin{array}{lllll}0 & 1 & 2 & 3 & 4\end{array}$

$\begin{array}{lllll}0 & 1 & 2 & 3 & 4\end{array}$

$\begin{array}{lllll}0 & 1 & 2 & 3 & 4\end{array}$

\section{4}

$\begin{array}{lllll}0 & 1 & 2 & 3 & 4\end{array}$

$\begin{array}{lllll}0 & 1 & 2 & 3 & 4\end{array}$


16. tried to keep my mind off things

that were upsetting me.

$\begin{array}{lllll}0 & 1 & 2 & 3 & 4\end{array}$

17. sought reassurance and emotional

support from others.

$\begin{array}{lllll}0 & 1 & 2 & 3 & 4\end{array}$

18. thought about how my problems were

caused by other people.

$\begin{array}{lllll}0 & 1 & 2 & 3 & 4\end{array}$

19. cried, even if someone else was around.

$\begin{array}{lllll}0 & 1 & 2 & 3 & 4\end{array}$

20. looked for how I could grow and learn

through my situation.

$\begin{array}{lllll}0 & 1 & 2 & 3 & 4\end{array}$

21. told myself that other people have

problems like mine.

$\begin{array}{lllll}0 & 1 & 2 & 3 & 4\end{array}$

22. done things to keep busy

or active (eg., exercised, went out).

23. held in my feelings.

24. daydreamed about how things may turn out.

25. tried to act as if I wasn't feeling bad.

26. taken steps to overcome the problem.

27. turned to God or my faith.

$\begin{array}{lllll}0 & 1 & 2 & 3 & 4 \\ 0 & 1 & 2 & 3 & 4 \\ 0 & 1 & 2 & 3 & 4 \\ 0 & 1 & 2 & 3 & 4 \\ 0 & 1 & 2 & 3 & 4 \\ 0 & 1 & 2 & 3 & 4\end{array}$




\section{MAAS}

Instructions: Below is a collection of statements about your everyday experiences. Using the 1 - 6 scale below, please indicate how frequently or infrequently you currently have each experience. Please answer according to what really reflects your experience, and not what you think your experience should be. Please treat each item separately from every other item.

$\begin{array}{cccccc}\begin{array}{c}\text { Almost } \\ \text { Always }\end{array} & \begin{array}{c}\text { Very } \\ \text { Frequently }\end{array} & \begin{array}{c}\text { Somewhat } \\ \text { Frequently }\end{array} & \begin{array}{c}\text { Somewhat } \\ \text { Infrequently }\end{array} & \begin{array}{c}\text { Very } \\ \text { Infrequently }\end{array} & \begin{array}{c}\text { Almost } \\ \text { Never }\end{array} \\ 1 & 2 & 3 & 4 & 5 & 6\end{array}$

1. I could be experiencing some emotion and not be conscious of it until some time later.

$\begin{array}{llllll}1 & 2 & 3 & 4 & 5 & 6\end{array}$

1. I break or spill things because of carelessness, not paying attention, or thinking of something else.

$\begin{array}{llllll}1 & 2 & 3 & 4 & 5 & 6\end{array}$

2. I find it difficult to stay focused on what's happening in the present.

$\begin{array}{llllll}1 & 2 & 3 & 4 & 5 & 6\end{array}$

3. I tend to walk quickly to get where I'm going, without paying attention to what I experience along the way.

$\begin{array}{llllll}1 & 2 & 3 & 4 & 5 & 6\end{array}$

4. I tend not to notice feelings of physical tension or discomfort until they really grab my attention.

$\begin{array}{llllll}1 & 2 & 3 & 4 & 5 & 6\end{array}$

5. I forget a person's name almost as soon as I've been told it for the first time.

$\begin{array}{llllll}1 & 2 & 3 & 4 & 5 & 6\end{array}$

6. It seems I am "running on automatic", without much Awareness of what I'm doing.

$\begin{array}{llllll}1 & 2 & 3 & 4 & 5 & 6\end{array}$

7. I rush through activities without being really attentive to them.

$\begin{array}{llllll}1 & 2 & 3 & 4 & 5 & 6\end{array}$

8. I get so focused on the goal I want to achieve that I lose touch with what I'm doing right now to get there.

$\begin{array}{llllll}1 & 2 & 3 & 4 & 5 & 6\end{array}$

9. I do jobs or tasks automatically, without being aware of what I'm doing.

$\begin{array}{llllll}1 & 2 & 3 & 4 & 5 & 6\end{array}$

10. I find myself listening to something with one ear, doing something else at the same time.

$\begin{array}{llllll}1 & 2 & 3 & 4 & 5 & 6\end{array}$




\section{SWL}

Instructions: Using the scale following each statement, please indicate the extent to which, on the whole, you agree or disagree with each one.

1. In most ways, my life is close to my ideal.

$\begin{array}{ccccccc}-3 & -2 & -1 & 0 & 1 & 2 & 3 \\ \text { Strongly } & \text { Moderately } & \text { Mildly } & \text { Neutral } & \text { Mildly } & \text { Moderately } & \text { Strongly } \\ \text { Disagree } & \text { Disagree } & \text { Disagree } & & \text { Agree } & \text { Agree } & \text { Agree }\end{array}$

2. If I could live my life over, I would change almost nothing.

$\begin{array}{ccccccc}-3 & -2 & -1 & 0 & 1 & 2 & 3 \\ \text { Strongly } & \text { Moderately } & \text { Mildly } & \text { Neutral } & \text { Mildly } & \text { Moderately } & \text { Strongly } \\ \text { Disagree } & \text { Disagree } & \text { Disagree } & & \text { Agree } & \text { Agree } & \text { Agree }\end{array}$

3. I am satisfied with my life.

$\begin{array}{ccccccc}-3 & -2 & -1 & 0 & 1 & 2 & 3 \\ \text { Strongly } & \text { Moderately } & \text { Mildly } & \text { Neutral } & \text { Mildly } & \text { Moderately } & \text { Strongly } \\ \text { Disagree } & \text { Disagree } & \text { Disagree } & & \text { Agree } & \text { Agree } & \text { Agree }\end{array}$

4. So far I have gotten the important things I want in life.

$\begin{array}{ccccccc}-3 & -2 & -1 & 0 & 1 & 2 & 3 \\ \text { Strongly } & \text { Moderately } & \text { Mildly } & \text { Neutral } & \text { Mildly } & \text { Moderately } & \text { Strongly } \\ \text { Disagree } & \text { Disagree } & \text { Disagree } & & \text { Agree } & \text { Agree } & \text { Agree }\end{array}$

5. The conditions of my life are excellent.

$\begin{array}{ccccccc}-3 & -2 & -1 & 0 & 1 & 2 & 3 \\ \text { Strongly } & \text { Moderately } & \text { Mildly } & \text { Neutral } & \text { Mildly } & \text { Moderately } & \text { Strongly } \\ \text { Disagree } & \text { Disagree } & \text { Disagree } & & \text { Agree } & \text { Agree } & \text { Agree }\end{array}$


Use the list below to answer the question:

In general, how happy or unhappy do you usually feel?

Circle the number beside the statement that best describes how happy you feel, on average.

$9 \quad$ Very happy (feeling really good, elated!)

8 Pretty happy (spirits high, feeling good)

$7 \quad$ Mildly happy (feeling fairly good and somewhat cheerful)

$6 \quad$ Slightly happy (just a bit more happy than neutral)

$5 \quad$ Neutral (not particularly happy or unhappy)

$4 \quad$ Slightly unhappy (just a bit more unhappy than neutral)

3 Mildly unhappy (just a bit low)

2 Pretty unhappy (somewhat 'blue', spirits are down)

1 Very unhappy (depressed, spirits very low)

$0 \quad$ Extremely unhappy (utterly depressed, completely down)

For each of the following statements, circle the number on the scale below that comes closest to how you feel.

(a) In general, how satisfied or dissatisfied are you with your life as a whole right now?

$\begin{array}{lllllllllll}0 & 1 & 2 & 3 & 4 & 5 & 6 & 7 & 8 & 9 & 10\end{array}$

Completely Dissatisfied

Neutral

Completely Satisfied

(b) In general, how satisfied or dissatisfied are you with your physical health as it is right now?

$\begin{array}{lllllllllll}0 & 1 & 2 & 3 & 4 & 5 & 6 & 7 & 8 & 9 & 10\end{array}$

Completely Dissatisfied Neutral Completely Satisfied

(c) In general, how satisfied or dissatisfied are you with your work life as it is right now?

$\begin{array}{lllllllllll}0 & 1 & 2 & 3 & 4 & 5 & 6 & 7 & 8 & 9 & 10\end{array}$

Completely Dissatisfied Neutral Completely Satisfied


(d) In general, how satisfied or dissatisfied are you with your social life as it is right now?

$\begin{array}{lllllllllll}0 & 1 & 2 & 3 & 4 & 5 & 6 & 7 & 8 & 9 & 10\end{array}$

Completely Dissatisfied

Neutral

Completely Satisfied

(e) In general, how satisfied or dissatisfied are you with your home life as it is right now?

$\begin{array}{lllllllllll}0 & 1 & 2 & 3 & 4 & 5 & 6 & 7 & 8 & 9 & 10\end{array}$

Completely Dissatisfied Neutral Completely Satisfied

Consider how you're feeling a bit further. On average, what percent of the time do you feel happy? What percent of the time do you feel unhappy? What percent of the time do you feel neutral (neither happy nor unhappy)? Write down your best estimates, as well as you can, in the spaces below. Make sure the 3 numbers add up to equal $100 \%$ of the time:

ON AVERAGE:

The percent of time I feel happy

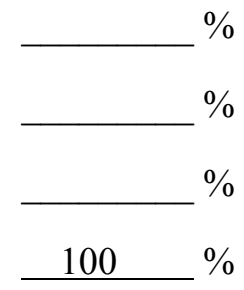

The percent of time I feel unhappy

$\%$

The percent of time I feel neutral

TOTAL

100 


\begin{abstract}
$\underline{\text { AASQ }}$
The following is a list of situations that you might encounter at one time or another. Please imagine yourself in each situation, and then indicate how threatening, as well as how distressing you would find each of these events. We will also be asking you to indicate your thoughts concerning each of these situations. Please note that there are no right answers for each question we are simply looking for your first reaction to each of these situations.

1) Your professor hands back last week's assignments to everyone but you, and then asks you to stay after class.
\end{abstract}

a. How threatening would this situation be for you?

1

Not at all

b. How distressing would this situation be for you?

1

2

3

4

Extremely

c. How much control do you think you would have over the outcome of this event?

1

No control
$2-3$

4

Complete control

d. What would you think the professor is most likely to say?

Your assignment was excellent - I just wanted to put a name to a face.

Your assignment was great, but there was one question where you could've done

better.

Is this your assignment? You forgot to put your name at the top.

I'm a little worried that you might not understand the material.

Your assignment was completely unsatisfactory - you should consider dropping this course. 
2) Imagine that you get home from class and there is a message from someone you're very close to (e.g., partner) who is away at another university that they really have to talk to you.

a. How threatening would this situation be to you?

1

Not at all

b. How distressing would this situation be for you?

1 2

Not at all
$2 \quad 3 \quad 4$

45

Extremely

c. How much control do you think you would have over the outcome of this event?

$\begin{array}{lllll}1 & 2 & 3 & 4 & 5\end{array}$

No control

Complete control

d. What would you think this person is most likely to say?

I really miss you - I just had to hear your voice.

I just got an A on my first mid-term.

I'm looking for an email address, do you have it?

I'm a little concerned about our relationship.

I've thought about it, and I don't think I need this relationship in my life.

3) CNN announces that an unknown number of planes have been hijacked simultaneously. You suddenly remember that your mother was flying to Minnesota for a business trip today.

a. How threatening would this situation be to you?
1
2
3
4
5

Not at all

Extremely 
b. How distressing would this situation be for you?

1

Not at all

$$
2
$$

3

4

\section{5}

Extremely

c. How much control do you think you would have over the outcome of this event?

$\begin{array}{lllll}1 & 2 & 3 & 4\end{array}$

No control

Complete control

d. What would you be most likely to think if you were in this situation?

The odds of my mom's plane being hijacked is incredibly small - I'm not worried about her at all.

I'm not worried yet, but I'd like to hear from her or at least get more information.

I'm a little bit apprehensive about this, but not so much that it disrupts my day

This is extremely worrisome - my mom very likely could be on one of those planes!

Oh my gosh! - I just know my mom is on one of those planes and is doomed.

\section{4) You and your partner are at a bar, and he sees you talking to a male friend. The next day, he does not return your calls.}

a. How threatening would this situation be for you?

$\begin{array}{lllll}1 & 2 & 3 & 5\end{array}$

Not at all

Extremely

b. How distressing would this situation be for you?

$\begin{array}{lllll}1 & 2 & 3 & 5\end{array}$

Not at all

Extremely

c. How much control do you think you would have over the outcome of this event?

$\begin{array}{lllll}1 & 2 & 3 & 4 & 5\end{array}$

None at all

Complete control 
d. What would you be most likely to think in this situation?

He's probably just out with his friends and hasn't had a chance to call me back yet. I'm not worried yet, I'll wait a while longer to see if he calls.

He's probably a little mad/jealous, but I'm sure he'll call back soon.

I'm a little worried that he is not going to call.

I should have known that speaking to that guy would make him angry, I am worried that he's going to break up with me.

\section{5) Most of your friends have left town to attend other universities.}

a. How threatening would this situation be for you?

1

Not at all

b. How distressing would this situation be for you?

1 2 3

4

Not at all
2

3
4

Extremely

c. How much control do you think you would have over the outcome of this event?

1

No control
2 3 4 Complete control

d. What would you think is most likely to happen to these friendships?

They will grow stronger.

They will get a little better.

They won't change at all.

They will get a little worse.

They will likely fade away. 
6) There is an outbreak of smallpox, and the government is only able to immunize a portion of the population. The criteria for immunization have not been disclosed.

a. How threatening would this situation be to you?

1

Not at all

b. How distressing would this situation be for you?

1

2

2

3

4
Not at all

Extremely

c. How much control do you think you would have over the outcome of this event?

1 2

No control

d. What would you be most likely to think of this possible outbreak of smallpox?

I'm sure that I'm safe - I will be immunized

Though I can't guarantee it, I'm fairly certain I'll be safe

It's hard to say either way what will happen

Knowing my luck, I probably won't meet the criteria - I might be in danger

I'm positive that I won't be picked - my life is in serious jeopardy

7) While doing a presentation, you notice a couple of students at the back of the class laughing.

a. How threatening would this situation be to you?
1
2
3
4
5

Not at all

Extremely

b. How distressing would this situation be for you?
1
2
3
4
5

Not at all

Extremely 
c. How much control do you think you would have over the outcome of this event?

1

No control
2
4

Complete control

d. What would you think that they would be most likely laughing about?

I must have said something funny - this presentation is going rather well.

Something that happened in the last person's presentation.

Something that happened last night and has nothing to do with my presentation.

My presentation in general - it's probably not that great.

I must look like a complete idiot up here, who has no idea what they are talking

about.

8) You have a friend visiting from out of town and you inform your partner that you won't be able to spend much time with him for a couple of days. Your partner expresses concern that your friends are taking up a lot of your time, and that he hasn't been able to see you much lately.

a. How threatening would this situation be for you?

$\begin{array}{lllll}1 & 2 & 3 & 4 & 5\end{array}$

Not at all Extremely

b. How distressing would this situation be for you?

$\begin{array}{lllll}1 & 2 & 3 & 4 & 5\end{array}$

Not at all Extremely

c. How much control do you think you would have over the outcome of this event?

$\begin{array}{lllll}1 & 2 & 3 & 4 & 5\end{array}$

Not at all Complete control 
d. What would you be most likely to think in this situation?

_ He really cares about me and just wants to spend as much time as possible together. He's just feeling left out. It's too bad that we can't spend much time together lately, but I'm sure he'll understand.

I'm worried that if I don't devote more time to him, this may negatively affect our relationship.

I'm concerned that if this keeps up, he may consider breaking up with me.

9) You are at health services for what you think is something minor. After the initial exam you glimpse of the nurse who just examined you speaking with your physician. They both appear to be concerned.

a. How threatening would this situation be to you?

$\begin{array}{lllll}1 & 2 & 3 & 4\end{array}$

Not at all

Extremely

b. How distressing would this situation be for you?

$\begin{array}{lllll}1 & 2 & 3 & 4 & 5\end{array}$

Not at all Extremely

c. How much control do you think you would have over the outcome of this event?

$\begin{array}{lllll}1 & 2 & 3 & 4 & 5\end{array}$

No control Complete control

d. What would you be most likely to think that is happening?

They are discussing a matter unrelated to my problem

They might be talking about me, but I'm sure it's nothing

Hmm....they look pretty serious. I wonder what that's about?

That doesn't look good - maybe the nurse suspects something?

This is definitely bad news - I've got something really serious wrong with me. 
10) You overhear a discussion about a party that your friends went to last week; however you are only hearing about it now.

a. How threatening would this situation be to you?

$\begin{array}{lllll}1 & 2 & 3 & 5\end{array}$

Not at all

Extremely

b. How distressing would this situation be for you?

$\begin{array}{lllll}1 & 2 & 3 & 4 & 5\end{array}$

$\begin{array}{ll}\text { Not at all Extremely } & \end{array}$

c. How much control do you think you would have over the outcome of this event?

$\begin{array}{lllll}1 & 2 & 3 & 4 & 5\end{array}$

No control Complete control

d. What would you think is the most likely reason that you didn't get invited along?

It was my own fault - I was at the library all night and they couldn't reach me.

My friends are forgetful, but it doesn't mean anything.

I must've mentioned a few days earlier that I didn't feel like going, so they didn't ask.

This has happened a few times now - I must have done something wrong.

I'm certain that I've fallen out of favour with my friends.

11) You have asked someone out on a date and they said "yes"; but, the next time you see them they seem distracted, in a bad mood, and essentially ignore you.

a. How threatening would this situation be to you?

$\begin{array}{lllll}1 & 2 & 3 & 4 & 5\end{array}$

Not at all

Extremely

b. How distressing would this situation be for you?

1

2

3

4

5

Not at all

Extremely 
c. How much control do you think you would have over the outcome of this event?

1

No control
2 3 4 Complete control

d. What would think is the most likely reason that they are acting this way?

No big deal - they are just in a bad mood, and it has nothing to do with me.

They are in a bad mood unrelated to me, but maybe I aggravated things but appearing suddenly.

It's hard to say... it could be about me, but maybe not.

The person is now unsure about me and is feeling awkward.

They didn't really want to say "yes" to the date in first place and are trying to let me know.

12) You are flipping around on the television when you notice that a severe weather warning has been issued for your area with reports of a tornado having touched down just west of your town.

a. How threatening would this situation be to you?

$\begin{array}{lllll}1 & 2 & 3 & 4 & 5\end{array}$

Not at all

Extremely

b. How distressing would this situation be for you?

1

$$
2
$$

3

Not at all
4 5 Extremely

c. How much control do you think you would have over the outcome of this event?

$\begin{array}{lllll}1 & 2 & 3 & 4 & 5\end{array}$

No control Complete control 
d. What would you be most likely to think of the threat of a potential tornado?

Excellent - I'll get to watch a great storm

It's probably nothing, but I'll keep an eye out

Hmm....while I'm concerned, it still doesn't call for action

I don't have a good feeling about this at all - I'm pretty sure I'm in danger.

This is horrible - this could pose a real threat to my life!

13) You're going to meet your partner's parents for the first time. Your partner tells you that he doesn't particularly like the outfit you are wearing to meet them, and suggests that you change.

a. How threatening would this situation be for you?

$\begin{array}{lllll}1 & 2 & 3 & 4 & 5\end{array}$

$\begin{array}{ll}\text { Not at all Extremely } & \end{array}$

b. How distressing would this situation be for you?

$\begin{array}{lllll}1 & 2 & 3 & 4\end{array}$

Not at all Extremely

c. How much control do you think you would have over the outcome of this event?

$\begin{array}{lllll}1 & 2 & 3 & 4 & 5\end{array}$

Not at all Complete control

d. What would you most likely think in this situation?

He must really be concerned about his parents' opinions.

He's just nervous about the meeting and wants me to make a good first impression. I appreciate his input but I'll just wear whatever makes me feel most comfortable. I must not look very good in this outfit.

He is embarrassed to introduce me to his parents. 
14) The supervisor at your part-time job calls and asks you to come in to discuss your most recent evaluation.

a. How threatening would this situation be to you?

$\begin{array}{lllll}1 & 2 & 3 & 4 & 5\end{array}$

Not at all

Extremely

b. How distressing would this situation be for you?

$\begin{array}{lllll}1 & 2 & 3 & 4 & 5\end{array}$

Not at all

Extremely

c. How much control do you think you would have over the outcome of this event?

$\begin{array}{lllll}1 & 2 & 3 & 4 & 5\end{array}$

No control Complete control

d. What would you think they are most likely to say?

Excellent job! - We would like to give you a raise

You are doing well - keep it up.

So far so good, but there is room for improvement

There are some serious problems here.

Things just aren't working out - we have to let you go.

15) Your first-year seminar instructor has asked a group of fellow students to participate in a student/teacher liaison committee but has not mentioned it to you.

a. How threatening would this situation be to you?

$\begin{array}{lllll}1 & 2 & 3 & 4 & 5\end{array}$

Not at all

Extremely

b. How distressing would this situation be for you?

1

2

3

4

5

Not at all

Extremely 
c. How much control do you think you would have over the outcome of this event?

1

No control
2
4

Complete control

d. What would you think is the most likely reason that it wasn't mentioned to you?

The instructor wanted to give the opportunity to students who really needed it.

I was most likely considered, but in the end went with someone else for whatever

reason

The students were just picked at random

I'm not completely sure, but sometimes I think the instructor believes my ideas are

weird

The instructor thinks that I'm not that intelligent, so he didn't bother asking me

16) You receive an unexpected message on your machine from student health services asking you to call the office after having gone in for routine check up/tests the week before.

a. How threatening would this situation be to you?

$\begin{array}{lllll}1 & 2 & 3 & 4\end{array}$

Not at all

Extremely

b. How distressing would this situation be for you?

$\begin{array}{lllll}1 & 2 & 3 & 4 & 5\end{array}$

Not at all

Extremely

c. How much control do you think you would have over the outcome of this event?

$\begin{array}{lllll}1 & 2 & 3 & 5\end{array}$

No control

Complete control 
d. What would you think is the most likely reason that they have asked you to call the office?

They just wanted to let me know that my test results came back normal.

My tests came back normal, but I need to schedule more tests for Spring.

They probably just need to confirm my health number for their records.

Something of concern probably showed up in my test results - this could be bad.

There is obviously something seriously wrong - why would they bother calling?

17) You go to take money out of your account, but your grant/scholarship/student loan check has not been processed by the bank / deposited by the university. Everyone else has his or hers.

a. How threatening would this situation be to you?

$\begin{array}{lllll}1 & 2 & 3 & 5\end{array}$

Not at all Extremely

b. How distressing would this situation be for you?

$\begin{array}{lllll}1 & 2 & 3 & 4 & 5\end{array}$

Not at all Extremely

c. How much control do you think you would have over the outcome of this event?

$\begin{array}{lllll}1 & 2 & 3 & 4\end{array}$

No control Complete control

d. What would you be most likely to think if this happened?

No big deal - It must just be delayed due to an administrative error. I'm confident

it's on the way.

I should probably keep an eye on it, but I'm pretty sure everything is okay.

Something is obviously wrong and I'm quite worried.

Something has definitely gone wrong with my loan/scholarship - I'm going to be

broke for the year! 
18) You are having a disagreement with your partner over some relationship issues and after stating your views on the situation, he gets frustrated and stomps out of the house.

a. How threatening would this situation be for you?

1

2

3

4 5

Not at all

b. How distressing would this situation be for you?

1

2

3

4

5

Not at all

c. How much control do you think you would have over the outcome of this event?

1

2

3

4

5

Not at all

Complete control

d. What would you be most likely to think in this situation?

His anger over this argument was kind of excessive.

Sometimes our arguments get out of hand but I'm sure things will be fine.

I'm not sure what to think...

He's really mad at me, I'm worried about how things will be between us when he gets back.

I shouldn't have made him so angry, I'm sure he won't be back for a while.

19) There has been a recent wave of "sniper" killings in town over the past few months. There aren't any firm leads and the victims seem to be chosen at random.

a. How threatening would this situation be to you?
1
2
3
4
5

Not at all

Extremely 
b. How distressing would this situation be for you?

1

Not at all
2

3

4
5

Extremely

c. How much control do you think you would have over the outcome of this event?

1 2 3 4 5

No control

Complete control

d. What would you be most likely to think about these sniper shootings?

With all the extra police around the city, crime will probably go down.

The chance of this impacting my life is pretty low. I'm not that worried.

This situation isn't critical yet, but it could get worse

This is pretty scary - I'm worried for both myself and my family

It's only a matter of time before this hit really close to home - I'm terrified.

20) You get an anonymous spiteful letter left on your locker door / left on your desk.

a. How threatening would this situation be to you?

1

Not at all
3
4

Extremely

b. How distressing would this situation be for you?
1
2
3
4 5

Not at all

Extremely

c. How much control do you think you would have over the outcome of this event?

1

2

No control
3

4

Complete control

5

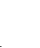

1


d. What would you be most likely to think if you were left this note?

My friends are such jokers - this is hilarious!

It's probably my friends playing a joke, but it's a pretty bad one.

I'm not really sure either way.

Does one of my "friends" really hate me?

This is devastating - I knew my friends have been irritated with me lately but I didn't

think it was this bad

21) One of your friends calls you to tell you that they saw your boyfriend/girlfriend out with another person on the weekend.

a. How threatening would this situation be to you?

$\begin{array}{lllll}1 & 2 & 3 & 4\end{array}$

Not at all Extremely

b. How distressing would this situation be for you?

$\begin{array}{lllll}1 & 2 & 3 & 4 & 5\end{array}$

Not at all Extremely

c. How much control do you think you would have over the outcome of this event?

$\begin{array}{lllll}1 & 2 & 3 & 4 & 5\end{array}$

No control Complete control

d. What would be most likely to be your first thought?

$\mathrm{He} /$ she goes out with friends all the time - I trust them completely

It's probably nothing - he/she probably had some friends in town.

It's strange that they didn't mention it, but maybe they just forgot.

This is not a good sign...I think he/she is losing interest in me.

I just knew that they were cheating on me. This relationship is over. 
22) You see a report on the news that there is plausible evidence that a "dirty bomb" has recently been smuggled into the country and may be used shortly.

a. How threatening would this situation be to you?

1

Not at all

b. How distressing would this situation be for you?

1 2

Not at all
3

4

Extremely

c. How much control do you think you would have over the outcome of this event?

1

No control
2 3

Complete control

d. What would you be most likely to think about the threat of this "dirty bomb"?

This is probably just another rumor - I'm going to ignore it.

It might be true, but I'm sure that I'm safe.

This may be something to take seriously, but I need more information first.

This really concerns me - I think I may be in danger

It is just a matter of time before we are hit with a devastating terrorist attack - I'm definitely in serious danger.

23) You were really drunk at a party last Friday night, the events are hazy but you know you've stepped on a few toes/made a fool of yourself and you have to face the class/ friends on Monday.

a. How threatening would this situation be to you?

$\begin{array}{lllll}1 & 2 & 3 & 5\end{array}$

Not at all

Extremely

b. How distressing would this situation be for you?

1

2

3

Not at all
45

Extremely 
c. How much control do you think you would have over the outcome of this event?

1

No control
2
4

Complete control

d. What would you be most likely to think that would happen?

My friends are going to think I'm a party animal and fun to hang out with.

I'm probably going to get made fun of but everyone will probably understand.

It's hard to say - some people might have been mildly offended, but maybe I'm

exaggerating

I'm pretty sure I've damaged a few friendships - things will definitely be awkward

for a while.

I'm going to be thought of as an idiot and I've definitely ruined a lot of friendships.

24) You are out to dinner at a restaurant with your partner, and he tells you he thinks you need to cut down on the amount of food you are eating.

a. How threatening would this situation be for you?

$\begin{array}{lllll}1 & 2 & 3 & 4 & 5\end{array}$

Not at all

Extremely

b. How distressing would this situation be for you?

$\begin{array}{lllll}1 & 2 & 3 & 4 & 5\end{array}$

Not at all Extremely

c. How much control do you think you would have over the outcome of this event?

$\begin{array}{lllll}1 & 2 & 3 & 4 & 5\end{array}$

Not at all Complete control 
d. What would you most likely think is the reason for his comment?

He is just looking out for my health, he really cares about me.

He probably just thinks that restaurant portions are too big these days, he didn't mean it as anything personal.

I'm not sure what to think...

He may be a little concerned that I will gain weight.

He thinks I am getting fat.

25) You are applying some sunscreen when you notice a small mass on your neck. Your physician performs a small biopsy and says it's probably nothing, but that he can't be sure until the test results come back in a week.

a. How threatening would this situation be to you?

1

Not at all

b. How distressing would this situation be for you?

1

2

3

4

Extremely

Not at all

Extremely

c. How much control do you think you would have over the outcome of this event?

1

No control
2

3

4

Complete control

d. What would be most likely to be your first thought?

It's definitely nothing to be concerned about

I'm a little concerned, but it's probably nothing

I'm not sure what to make of this

I'm pretty worried about this - I don't have a good feeling about it.

It's definitely something life-threatening- why else would they bother with a biopsy? 
26) A couple of weeks after you have decided to invest a significant portion of your student loan in stocks, the market begins to waiver.

a. How threatening would this situation be to you?

$\begin{array}{lllll}1 & 2 & 3 & 4 & 5\end{array}$

Not at all

Extremely

b. How distressing would this situation be for you?

$\begin{array}{lllll}1 & 2 & 3 & 4 & 5\end{array}$

Not at all

Extremely

c. How much control do you think you would have over the outcome of this event?

$\begin{array}{lllll}1 & 2 & 3 & 4 & 5\end{array}$

No control Complete control

d. What would you be most likely to think about this situation?

It is a pretty safe bet - In the long term things will work out

It is getting a bit risky, but I think I know what I'm doing

I'm going to wait and see but it could go either way.

Uh oh...I'm pretty worried that I could lose it all

What have I done? - this is going to ruin me for the year!

27) Someone you are very close to (e.g., partner) leaves to drive home and is supposed to call your when they get in. It's been a couple of hours and you haven't heard anything. There is no answer at their place.

a. How threatening would this situation be to you?

$\begin{array}{lllll}1 & 2 & 3 & 4 & 5\end{array}$

Not at all

Extremely

b. How distressing would this situation be for you?

1

2

Not at all
3

4

Extremely 
c. How much control do you think you would have over the outcome of this event?

1

No control
2 3 4 Complete control

d. What would you think is the most likely reason they haven't called?

I'm positive they just forgot to call and went to friend's house on the way home I'm a little surprised I haven't heard from them, but everything should be okay I don't know what to think...

This is not a good sign - something might have happened

I'm certain that something terrible has happened - I'm positive I would have heard from them by now.

28) You phone your department to see if your request to switch your major has gone through, but the person at the desk tells you that the matter cannot be discussed on the phone and that a letter has been mailed to you in this regard.

a. How threatening would this situation be to you?

$\begin{array}{lllll}1 & 2 & 3 & 4 & 5\end{array}$

Not at all Extremely

b. How distressing would this situation be for you?

$\begin{array}{lllll}1 & 2 & 3 & 4 & 5\end{array}$

Not at all Extremely

c. How much control do you think you would have over the outcome of this event?

$\begin{array}{lllll}1 & 2 & 3 & 4 & 5\end{array}$

No control Complete control 
d. What would you be most likely to think that has happened with your application?

This must be standard procedure for the department - everything is fine

I wish they could just tell me, but I'm pretty sure it's fine

That is odd - why can't they just tell me? Hmm...

That doesn't sound very good - it could be bad news

That does it - my transfer didn't go through. It's just a matter of time before I get my rejection letter.

29) You have heard reports that a group of youths has been beating and robbing individuals around shopping malls and bus stations. On the way to the bus-stop you notice that a group of kids is beginning to congregate in the adjacent parking lot.

a. How threatening would this situation be to you?

$\begin{array}{lllll}1 & 2 & 3 & 4 & 5\end{array}$

Not at all

Extremely

b. How distressing would this situation be for you?

$\begin{array}{lllll}1 & 2 & 3 & 4 & 5\end{array}$

Not at all Extremely

c. How much control do you think you would have over the outcome of this event?

$\begin{array}{lllll}1 & 2 & 3 & 4 & 5\end{array}$

No control Complete control

d. What would be most likely to think of this situation?

It's just a bunch of kids - I'm sure they are just hanging out and having a good time and aren't going to harm anyone.

I wonder if I recognize any of those. They seem to be ready to engage in some sort of activity.

What a big group of kids....I wonder what they are up to.

This is a bit scary - I hope this isn't the group I heard about it.

I'm terrified - That is the group of kids who have been stealing people's money - If I

go over there I'm sure to be attacked! 


\section{Appendix D: Study 1 Debriefing}

\section{What are we trying to learn in this research?}

This research examines the relationship between personal factors, appraisals, depressive symptoms and well-being. The questionnaires you completed assessed subjective well-being, depressive symptoms and mindfulness. We also asked you to provide information on the coping strategies you typically use, and how you tend to appraise yourself and different situations. We are interested in learning if positive self-appraisals and mindfulness are associated with the use of more effective coping strategies and general well-being. We are also interested in determining if reading and reflecting upon concepts related to mindfulness encourages people to view situations in a more positive way. To assess this, we asked you to think of a recent stressful event in your life, and to write about this situation using words related to either mindfulness, non-mindfulness, or to describe this event in your own words. We used these different sets of instructions in order to see if these words influenced how people evaluated different hypothetical situations in later questionnaires.

\section{Why is this important to scientists or the general public?}

Previous research has shown a relationship between people's appraisals of different situations, and how they cope with those situations. Less is known, however, about the specific factors that lead people to appraise events in different ways. Understanding individual difference variables (e.g., mindfulness) and self-appraisals (e.g., self-esteem, mastery) that might be associated with more positive appraisals of events is important, since these appraisals may predict enhanced coping abilities. If mindfulness and positive self-appraisals are related to effective coping and well-being, it might be helpful to focus on promoting the development of these skills in order to reduce stress and improve well-being.

\section{What are our hypotheses and predictions?}

We predict that individuals' self-appraisals (the way people evaluate themselves) will predict how they approach different situations, and will also predict how well they are able to cope with stressful situations. We also predict that mindfulness (e.g., the ability to live "in the moment", and to observe situations in an objective, non self-judgmental way) will be associated with more positive selfappraisals, coping and well-being.

\section{Is there anything I can do if I found this experiment to be emotionally upsetting?}

Yes. If you feel any distress or anxiety after participating in this study, please feel free to contact the Carleton University Health and Counseling Services at: 613-520-6674, or the Distress Centre of Ottawa and Region at 613-238-3311 (http://www.dcottawa.on.ca).

\section{What if I have questions later?}

If you have any remaining concerns, questions, or comments about the experiment, please feel free to contact Kelly-Lyn Christie (Principal Investigator), at: klchrist@connect.carleton.ca (613-520-2600, ext. 6312), Valerie Repta, at: vrepta@connect.carleton.ca, Dr. Hymie Anisman (Faculty Sponsor), at: 
hanisman@connect.carleton.ca, or Dr. Kimberly Matheson, at: kim_matheson@carleton.ca. Should you have any ethical concerns about this research, please contact Dr. Monique Sénéchal at: Monique_senechal@carleton.ca (613-520-2600 ext 1155). For other concerns, please contact Dr. Janet Mantler (Chair, Department of Psychology, 613-520-2600, ext. 2648, psychchair@carleton.ca).

\section{Thank you for participating in this research!}




\title{
Appendix E: Study 2 Student and Community Recruitment Notices
}

\author{
Student Recruitment Notice
}

Electronic Sign-up System Information

Study Name: Self-Appraisals and Situational Coping

Brief Abstract: This study examines how people appraise different situations and themselves, and how these appraisals influence coping strategies across a variety of situations.

Detailed Description: We are interested in exploring the relationship between personal characteristics (e.g., demographics, depressive symptoms, mindfulness), how individuals appraise a) themselves, and b) different types of situations, and the strategies individuals use for coping with different types of stressful situations. We are further interested in examining how these variables are related to well-being. The session requires completion of a questionnaire package regarding personal characteristics, appraisals, the use of various types of coping strategies, and well-being.

This study has received clearance by the Carleton University Psychology Research Ethics Board (Ethics number : 10-116).

Duration: 1 hour

Credits: $0.5 \%$ increase 


\section{Participate in a Study and Earn a \$5 Gift Card!}

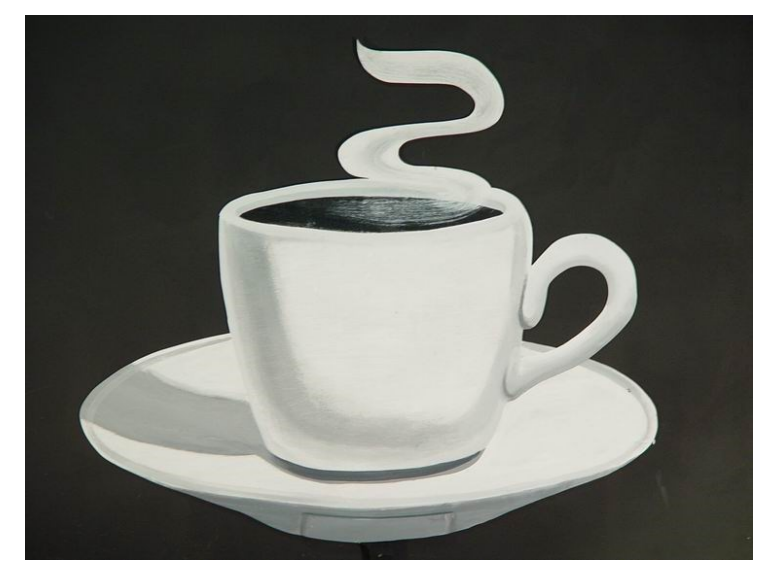

We are studying how individuals appraise different situations, coping strategies, and how stress and coping methods are related to well-being. You can help us with this research by participating online!

Participation requires completion of an online questionnaire package regarding personal characteristics, appraisals, coping strategies, and wellbeing, and will take approximately 1 hour of your time.

\section{Individuals who complete this online survey will receive a $\$ 5.00 \mathrm{Tim}$ Horton's gift certificate for their participation.}

For more information, or to participate in this study, please contact Kelly Christie, principal researcher, at klchrist@connect.carleton.ca, or visit: http://www.carleton.ca/copewell

\section{Thank You!}

This study has received clearance by the Carleton University Psychology Research Ethics Board (Ethics number : 10-116). 


\section{Appendix F: Study 2 Informed Consent}

The purpose of an informed consent is to ensure that you understand the purpose of the study and the nature of your involvement. The informed consent must provide sufficient information such that you have the opportunity to determine whether you wish to participate in the study.

This study has received clearance by the Carleton University Psychology Research Ethics Board (Ethics number: 10-116).

\section{Present study: Self-Appraisals and Situational Coping}

Research personnel. The following people are involved in this research project, and may be contacted at any time if you have questions or concerns:

Kelly-Lyn Christie (email: klchrist@connect.carleton.ca, phone: 613-520-2600, ext. 6312),

Valerie Repta (email: vrepta@connect.carleton.ca, phone 613-520-2600, ext. 7513),

Dr. Hymie Anisman (Faculty Sponsor, email: hanisman@connect.carleton.ca),

Dr. Kimberly Matheson (email: kim_matheson@carleton.ca ).

Should you have any ethical concerns about this research, please contact Dr. Monique Sénéchal, at : monique_senechal@carleton.ca (613-520-2600 ext. 1155). For any other concerns, please contact Dr. Janet Mantler (Chair, Department of Psychology, 613-520-2600, ext. 4171, psychchair@carleton.ca).

Purpose. The purpose of this study is to investigate the relationship between personal characteristics (e.g., demographics, mindfulness) and how individuals appraise different types of situations and themselves. We are interested in examining how these personal characteristics and appraisals influence the types of strategies people use when dealing with different types of stressful situations, as well as how these variables predict well-being.

Task requirements. We are asking you to fill out a number of questionnaires regarding your background (e.g., demographics), personal characteristics (e.g., how you cope with things in your life), and how you feel about yourself (e.g., self-appraisals, well-being). The questionnaires will take about 60 minutes to complete. Students: You will receive a $0.5 \%$ increase in your final grade of PSYC 1001, PSYC 1002, PSYC 2001 or PSYC 2002 for participating in this study.

Community participants: You will receive a $\$ 5.00$ Tim Horton's gift card for participating in this study. Testing will take place online.

Potential risk/discomfort. There are no physical risks to participating in this study. Some individuals might feel uncomfortable when asked to recall stressful situations or to answer personal, sensitive questions about the coping strategies they use or feelings of depression they may have experienced. If you feel any discomfort or distress, you may choose not to answer specific questions, and you will not be penalized in any way if you do this. The debriefing form at the end of the study provides contact information for local support services that you may contact if you need or want help.

Anonymity/Confidentiality. The data collected in this experiment are strictly confidential. All data are coded such that your name is not associated with the responses you provide. Any 
identifying information associated with your code will be confined to a single page that will be separated from your questionnaire, and kept in a separate, secured file by the research investigators, who will keep this information confidential. At the end of this study, we will be asking you for permission to keep your contact information on file for one year in order to contact you to ask if you'd be willing to participate in follow-up studies.

Right to withdraw. Your participation in this study is entirely voluntary. At any point during the study, you have the right to not complete certain questions or to withdraw from the study. If we ask a question and you do not wish to provide an answer, please leave the question blank on the online survey (e.g., do not select a response). You may then go on to the next question. Please note that you will not be penalized if you choose to leave some questions blank.

By clicking the "next" button you will be directed to the online questionnaire. If you proceed and complete the questionnaire, your may submit your responses by clicking the "submit" button. The data collected will be used in research publications and/or for teaching purposes.

When you click the "next" button and submit your data, it will be assumed that you have read the above description of the study concerning self-appraisals and situational coping, and have granted consent. However, should you wish to withdraw your data after submission we will grant that request immediately. 


\section{Appendix G: Study 2 Questionnaires}

\section{$\underline{\text { SCOPE }}$}

The purpose of this questionnaire is to find out how people generally deal with their problems or the stresses in their lives. The following are activities that you may have done. After each activity, please indicate the extent to which you have used this as a way of dealing with problems or stresses in recent weeks. Select which of the five options describes you most closely by selecting the appropriate box.

\begin{tabular}{|c|c|c|c|c|c|c|}
\hline & $\begin{array}{l}\text { Ordinarily, in recent weeks, I } \\
\text { have... }\end{array}$ & Never & Rarely & Sometimes & Often & $\begin{array}{l}\text { Almost } \\
\text { Always }\end{array}$ \\
\hline 1 & $\begin{array}{l}\text { accepted that there was nothing I } \\
\text { could do to change my situation }\end{array}$ & & & & & \\
\hline 2 & $\begin{array}{l}\text { tried to just take whatever came } \\
\text { my way }\end{array}$ & & & & & \\
\hline 3 & $\begin{array}{l}\text { talked with friends or relatives } \\
\text { about my problems }\end{array}$ & & & & & \\
\hline 4 & $\begin{array}{l}\text { tried to do things which I } \\
\text { typically enjoy }\end{array}$ & & & & & \\
\hline 5 & $\begin{array}{l}\text { sought out information that } \\
\text { would help me resolve my } \\
\text { problems }\end{array}$ & & & & & \\
\hline 6 & $\begin{array}{l}\text { blamed others for creating my } \\
\text { problems or making them worse }\end{array}$ & & & & & \\
\hline 7 & $\begin{array}{l}\text { sought the advice of others to } \\
\text { resolve my problems }\end{array}$ & & & & & \\
\hline 8 & $\begin{array}{l}\text { focused on the present moment } \\
\text { rather than the past or future }\end{array}$ & & & & & \\
\hline 9 & blamed myself for my problems & & & & & \\
\hline 10 & exercised & & & & & \\
\hline 11 & $\begin{array}{l}\text { fantasized or thought about } \\
\text { unreal things (e.g., the perfect } \\
\text { revenge, or winning a million } \\
\text { dollars) to feel better }\end{array}$ & & & & & \\
\hline 12 & $\begin{array}{l}\text { been very emotional compared to } \\
\text { my usual self }\end{array}$ & & & & & \\
\hline 13 & $\begin{array}{l}\text { gone over my problems in my } \\
\text { mind over and over again }\end{array}$ & & & & & \\
\hline 14 & asked others for help & & & & & \\
\hline
\end{tabular}




\begin{tabular}{|c|c|c|c|c|c|c|}
\hline & $\begin{array}{l}\text { Ordinarily, in recent weeks, I } \\
\text { have... }\end{array}$ & Never & Rarely & Sometimes & Often & $\begin{array}{l}\text { Almost } \\
\text { Always }\end{array}$ \\
\hline 15 & thought about my problems a lot & & & & & \\
\hline 16 & $\begin{array}{l}\text { became involved in recreation or } \\
\text { pleasure activities }\end{array}$ & & & & & \\
\hline 17 & $\begin{array}{l}\text { paid attention to how I was } \\
\text { reacting to the situation, without } \\
\text { judging myself }\end{array}$ & & & & & \\
\hline 18 & worried about my problems a lot & & & & & \\
\hline 19 & $\begin{array}{l}\text { tried to keep my mind off things } \\
\text { that are upsetting me }\end{array}$ & & & & & \\
\hline 20 & $\begin{array}{l}\text { tried to distract myself from my } \\
\text { troubles }\end{array}$ & & & & & \\
\hline 21 & $\begin{array}{l}\text { avoided thinking about my } \\
\text { problems }\end{array}$ & & & & & \\
\hline 22 & $\begin{array}{l}\text { made plans to overcome my } \\
\text { problems }\end{array}$ & & & & & \\
\hline 23 & $\begin{array}{l}\text { observed my thoughts rather than } \\
\text { trying to change them }\end{array}$ & & & & & \\
\hline 24 & told jokes about my situation & & & & & \\
\hline 25 & $\begin{array}{l}\text { thought a lot about who is } \\
\text { responsible for my problems } \\
\text { (besides yourself) }\end{array}$ & & & & & \\
\hline 26 & $\begin{array}{l}\text { shared humorous stories etc. to } \\
\text { cheer myself and others up }\end{array}$ & & & & & \\
\hline 27 & $\begin{array}{l}\text { told myself that other people } \\
\text { have dealt with problems such as } \\
\text { mine }\end{array}$ & & & & & \\
\hline 28 & $\begin{array}{l}\text { thought a lot about how I have } \\
\text { brought my problems on myself }\end{array}$ & & & & & \\
\hline 29 & $\begin{array}{l}\text { decided to wait and see how } \\
\text { things turn out }\end{array}$ & & & & & \\
\hline 30 & $\begin{array}{l}\text { reminded myself to breathe and } \\
\text { focus on the present moment }\end{array}$ & & & & & \\
\hline 31 & $\begin{array}{l}\text { wished the situation would go } \\
\text { away or be over with }\end{array}$ & & & & & \\
\hline 32 & $\begin{array}{l}\text { decided that my current } \\
\text { problems are a result of my own } \\
\text { past actions }\end{array}$ & & & & & \\
\hline
\end{tabular}




\begin{tabular}{|c|c|c|c|c|c|c|}
\hline & $\begin{array}{l}\text { Ordinarily, in recent weeks, I } \\
\text { have... }\end{array}$ & Never & Rarely & Sometimes & Often & $\begin{array}{l}\text { Almost } \\
\text { Always }\end{array}$ \\
\hline 33 & gone shopping & & & & & \\
\hline 34 & $\begin{array}{l}\text { asserted myself and taken } \\
\text { positive action on problems that } \\
\text { are getting me down }\end{array}$ & & & & & \\
\hline 35 & $\begin{array}{l}\text { sought reassurance and moral } \\
\text { support from others }\end{array}$ & & & & & \\
\hline 36 & resigned myself to my problems & & & & & \\
\hline 37 & $\begin{array}{l}\text { made an effort to accept my } \\
\text { feelings and thoughts for what } \\
\text { they were, rather than trying to } \\
\text { control them }\end{array}$ & & & & & \\
\hline 38 & $\begin{array}{l}\text { thought about how my problems } \\
\text { have been caused by other } \\
\text { people }\end{array}$ & & & & & \\
\hline 39 & $\begin{array}{l}\text { daydreamed about how things } \\
\text { may turn out }\end{array}$ & & & & & \\
\hline 40 & $\begin{array}{l}\text { been very emotional in how I } \\
\text { react, even to little things }\end{array}$ & & & & & \\
\hline 41 & $\begin{array}{l}\text { taken time simply to breathe and } \\
\text { calm myself down }\end{array}$ & & & & & \\
\hline 42 & $\begin{array}{l}\text { decided that I can grow and learn } \\
\text { through my problems }\end{array}$ & & & & & \\
\hline 43 & $\begin{array}{l}\text { told myself that other people } \\
\text { have problems like my own }\end{array}$ & & & & & \\
\hline 44 & $\begin{array}{l}\text { wished I was a stronger person } \\
\text { or better at dealing with } \\
\text { problems }\end{array}$ & & & & & \\
\hline 45 & $\begin{array}{l}\text { looked for how I can learn } \\
\text { something out of my bad } \\
\text { situation }\end{array}$ & & & & & \\
\hline 46 & asked for God's guidance & & & & & \\
\hline 47 & $\begin{array}{l}\text { kept my feelings bottled up } \\
\text { inside }\end{array}$ & & & & & \\
\hline 48 & $\begin{array}{l}\text { found myself crying more than } \\
\text { usual }\end{array}$ & & & & & \\
\hline 49 & tried to act as if I wasn't upset & & & & & \\
\hline
\end{tabular}




\begin{tabular}{|l|l|l|l|l|l|}
\hline $\begin{array}{l}\text { Ordinarily, in recent weeks, I } \\
\text { have... }\end{array}$ & Never & Rarely & Sometimes & Often & $\begin{array}{l}\text { Almost } \\
\text { Always }\end{array}$ \\
\hline 50 prayed for help & & & & & \\
\hline $\begin{array}{l}\text { became aware of my thoughts } \\
\text { and feelings, without trying to } \\
\text { change them or get carried away } \\
\text { by them }\end{array}$ & & & & & \\
\hline 52 gone out & & & & & \\
\hline 53 held in my feelings & & & & \\
\hline $54 \begin{array}{l}\text { tried to act as if I wasn't feeling } \\
\text { bad }\end{array}$ & & & & & \\
\hline 55 & $\begin{array}{l}\text { taken steps to overcome my } \\
\text { problems }\end{array}$ & & & & \\
\hline $56 \begin{array}{l}\text { made humorous comments or } \\
\text { wise cracks }\end{array}$ & & & & & \\
\hline $57 \begin{array}{l}\text { tried to keep the situation in } \\
\text { perspective and not overreact }\end{array}$ & & & & & \\
\hline $58 \begin{array}{l}\text { told others that I was depressed } \\
\text { or emotionally upset }\end{array}$ & & & & & \\
\hline
\end{tabular}




\section{SCOPE - Personal / Interpersonal Stress}

The purpose of this questionnaire is to find out how people deal with personal problems or stresses in their lives. The following are activities that you may have done in response to such events. Please take a moment to think of a recent personal stress that you have experienced (e.g., an argument with a friend or partner, etc.). After each activity, please indicate the extent to which you have used this strategy as a way of dealing with this personally stressful event.

To cope with this personal stressor, I have:

2. accepted that there is nothing I could do to change my situation.

2. blamed myself for my problem.

3. told others that I was really upset.

4. asked others for help or advice.

5. spent a lot of time thinking about my problem.

6. taken time for recreation or pleasure activities.

7. made plans to overcome my concerns or problem.

8. focused on the present moment rather than the past or future

9. avoided thinking about my problem.

10. told jokes about my situation.

11. thought a lot about who was responsible for my problem (besides me).

12. worried about my problem a lot.

13. made humorous comments or stories about my situation.

14. wished the situation would just go away or be over with

15. thought a lot about how I brought my problem on myself.

16. paid attention to how I was reacting to the situation, without judging myself.

17. decided to wait and see how things turned out.

18. tried to keep my mind off things that were upsetting me. $\begin{array}{llll}0 & 1 & 2 & 3\end{array}$

\section{Not at all Somewhat Totally \\ Not at all Somewhat Totally}

$\begin{array}{lllll}0 & 1 & 2 & 3 & 4 \\ 0 & 1 & 2 & 3 & 4 \\ 0 & 1 & 2 & 3 & 4\end{array}$

$\begin{array}{lllll}0 & 1 & 2 & 3 & 4\end{array}$

$\begin{array}{lllll}0 & 1 & 2 & 3 & 4\end{array}$

$\begin{array}{lllll}0 & 1 & 2 & 3 & 4\end{array}$

$\begin{array}{lllll}0 & 1 & 2 & 3 & 4\end{array}$

$\begin{array}{lllll}0 & 1 & 2 & 3 & 4\end{array}$

$\begin{array}{lllll}0 & 1 & 2 & 3 & 4\end{array}$

$\begin{array}{lllll}0 & 1 & 2 & 3 & 4\end{array}$

4

4

4

4

4

4

4

4 4 
19. observed my thoughts rather than trying to change them. $\begin{array}{llllll}0 & 1 & 2 & 3 & 4\end{array}$

20. sought reassurance and emotional support from others. $\begin{array}{llllll}0 & 1 & 2 & 3 & 4\end{array}$

21. thought about how my problem was caused by other people.

22. cried, even if someone else was around.

$\begin{array}{lllll}0 & 1 & 2 & 3 & 4 \\ 0 & 1 & 2 & 3 & 4\end{array}$

23. became aware of my thoughts and feelings, without trying to change them or get carried away by them.

24. looked for how I could grow and learn through my situation.

$\begin{array}{lllll}0 & 1 & 2 & 3 & 4\end{array}$

25. told myself that other people have problems like mine. $\begin{array}{llllll}0 & 1 & 2 & 3 & 4\end{array}$

26. done things to keep busy or active (e.g., exercised, went out).

27. held in my feelings.

28. daydreamed about how things may turn out.

$\begin{array}{llll}1 & 2 & 3 & 4\end{array}$

29. tried to act as if I wasn't feeling bad.

$\begin{array}{lllll}0 & 1 & 2 & 3 & 4\end{array}$

30. reminded myself to breathe and focus on the present moment.

31. taken steps to overcome the problem.

32. turned to God or my faith.

$\begin{array}{lllll}0 & 1 & 2 & 3 & 4\end{array}$

$\begin{array}{lllll}0 & 1 & 2 & 3 & 4\end{array}$

$\begin{array}{lllll}0 & 1 & 2 & 3 & 4\end{array}$




\section{SCOPE - Financial Stress}

The purpose of this questionnaire is to find out how people deal with financial problems or stresses in their lives. The following are activities that you may have done in response to such events. Please take a moment to think of a recent financial stress that you have experienced (e.g., having to borrow money from someone, having unexpected expenses, going into debt, etc.). After each activity, please indicate the extent to which you have used this strategy as a way of dealing with this stressful event.

To cope with this financial stressor, I have:

Not at all $\begin{array}{llll}0 & 1 & 2 & 3\end{array}$

Somewhat
Totally

1. accepted that there is nothing I could do to change my situation.

2. blamed myself for my problem.

3. told others that I was really upset.

4. asked others for help or advice.

5. spent a lot of time thinking about my problem.

6. taken time for recreation or pleasure activities.

7. made plans to overcome my concerns or problem.

8. focused on the present moment rather than the past or future

9. avoided thinking about my problem.

10. told jokes about my situation.

11. thought a lot about who was responsible for my problem (besides me).

12. worried about my problem a lot.

13. made humorous comments or stories about my situation.

14. wished the situation would just go away or be over with

15. thought a lot about how I brought my problem on myself.

16. paid attention to how I was reacting to the situation, without judging myself.

17. decided to wait and see how things turned out.

18. tried to keep my mind off things that were upsetting me.

$\begin{array}{lllll}0 & 1 & 2 & 3 & 4 \\ 0 & 1 & 2 & 3 & 4 \\ 0 & 1 & 2 & 3 & 4 \\ 0 & 1 & 2 & 3 & 4 \\ 0 & 1 & 2 & 3 & 4 \\ 0 & 1 & 2 & 3 & 4 \\ 0 & 1 & 2 & 3 & 4\end{array}$

$\begin{array}{lllll}0 & 1 & 2 & 3 & 4\end{array}$

$\begin{array}{lllll}0 & 1 & 2 & 3 & 4\end{array}$

$\begin{array}{lllll}0 & 1 & 2 & 3 & 4\end{array}$

\section{4}

4

$\begin{array}{lllll}0 & 1 & 2 & 3 & 4\end{array}$

$\begin{array}{lllll}0 & 1 & 2 & 3 & 4\end{array}$

$\begin{array}{lllll}0 & 1 & 2 & 3 & 4\end{array}$

$\begin{array}{llll}1 & 2 & 3 & 4\end{array}$


19. observed my thoughts rather than trying to change them. $\begin{array}{llllll}0 & 1 & 2 & 3 & 4\end{array}$

20. sought reassurance and emotional support from others. $\begin{array}{llllll}0 & 1 & 2 & 3 & 4\end{array}$

21. thought about how my problem was caused by other people.

22. cried, even if someone else was around.

$\begin{array}{lllll}0 & 1 & 2 & 3 & 4 \\ 0 & 1 & 2 & 3 & 4\end{array}$

23. became aware of my thoughts and feelings, without trying to change them or get carried away by them.

24. looked for how I could grow and learn through my situation.

$\begin{array}{lllll}0 & 1 & 2 & 3 & 4\end{array}$

25. told myself that other people have problems like mine. $\begin{array}{llllll}0 & 1 & 2 & 3 & 4\end{array}$

26. done things to keep busy or active (e.g., exercised, went out).

27. held in my feelings.

28. daydreamed about how things may turn out.

$\begin{array}{llll}1 & 2 & 3 & 4\end{array}$

29. tried to act as if I wasn't feeling bad.

$\begin{array}{lllll}0 & 1 & 2 & 3 & 4\end{array}$

30. reminded myself to breathe and focus on the present moment.

31. taken steps to overcome the problem.

32. turned to God or my faith.

$\begin{array}{lllll}0 & 1 & 2 & 3 & 4\end{array}$

$\begin{array}{lllll}0 & 1 & 2 & 3 & 4\end{array}$

$\begin{array}{lllll}0 & 1 & 2 & 3 & 4\end{array}$




\section{SCOPE - Work / Academic Stress}

The purpose of this questionnaire is to find out how people deal with workplace or academic problems or stresses in their lives. The following are activities that you may have done in response to such events. Please take a moment to think of a recent work or academic stress that you have experienced (e.g., being turned down for a promotion, receiving a poor grade on an exam, being placed on probation at school or work, etc.). After each activity, please indicate the extent to which you have used this strategy as a way of dealing with this stressful event.

To cope with this academic/work stressor, I have:

$\begin{array}{ccccc}0 & 1 & 2 & 3 & 4 \\ \text { Notat all } & & \text { Somewhat } & & \text { Totally }\end{array}$

1. accepted that there is nothing I could do to change my situation.

2. blamed myself for my problem.

3. told others that I was really upset.

4. asked others for help or advice.

5. spent a lot of time thinking about my problem.

6. taken time for recreation or pleasure activities.

7. made plans to overcome my concerns or problem.

$\begin{array}{lllll}0 & 1 & 2 & 3 & 4 \\ 0 & 1 & 2 & 3 & 4 \\ 0 & 1 & 2 & 3 & 4 \\ 0 & 1 & 2 & 3 & 4 \\ 0 & 1 & 2 & 3 & 4 \\ 0 & 1 & 2 & 3 & 4 \\ 0 & 1 & 2 & 3 & 4\end{array}$

8. focused on the present moment rather than the past or future

9. avoided thinking about my problem.

10. told jokes about my situation.

0

11. thought a lot about who was responsible for my problem (besides me).

12. worried about my problem a lot.

$\begin{array}{lllll}0 & 1 & 2 & 3 & 4 \\ 0 & 1 & 2 & 3 & 4\end{array}$

13. made humorous comments or stories about my situation.

14. wished the situation would just go away or be over with

15. thought a lot about how I brought my problem on myself.

16. paid attention to how I was reacting to the situation, without judging myself.

17. decided to wait and see how things turned out.

18. tried to keep my mind off things that were upsetting me.

$\begin{array}{lllll}0 & 1 & 2 & 3 & 4 \\ 0 & 1 & 2 & 3 & 4 \\ 0 & 1 & 2 & 3 & 4\end{array}$


19. observed my thoughts rather than trying to change them. $\begin{array}{llllll}0 & 1 & 2 & 3 & 4\end{array}$

20. sought reassurance and emotional support from others. $\begin{array}{llllll}0 & 1 & 2 & 3 & 4\end{array}$

21. thought about how my problem was caused by other people.

22. cried, even if someone else was around.

$\begin{array}{lllll}0 & 1 & 2 & 3 & 4 \\ 0 & 1 & 2 & 3 & 4\end{array}$

23. became aware of my thoughts and feelings, without trying to change them or get carried away by them.

24. looked for how I could grow and learn through my situation.

$\begin{array}{lllll}0 & 1 & 2 & 3 & 4\end{array}$

25. told myself that other people have problems like mine. $\begin{array}{llllll}0 & 1 & 2 & 3 & 4\end{array}$

26. done things to keep busy or active (e.g., exercised, went out).

27. held in my feelings.

28. daydreamed about how things may turn out.

$\begin{array}{llll}1 & 2 & 3 & 4\end{array}$

29. tried to act as if I wasn't feeling bad.

$\begin{array}{lllll}0 & 1 & 2 & 3 & 4\end{array}$

30. reminded myself to breathe and focus on the present moment.

31. taken steps to overcome the problem.

32. turned to God or my faith.

$\begin{array}{lllll}0 & 1 & 2 & 3 & 4\end{array}$

$\begin{array}{lllll}0 & 1 & 2 & 3 & 4\end{array}$

$\begin{array}{lllll}0 & 1 & 2 & 3 & 4\end{array}$




\section{Appendix H: Study 2 Debriefing}

\section{What are we trying to learn in this research?}

This research examines the relationship between personal factors (i.e., self-appraisals, mindfulness), depressive symptoms and well-being. The questionnaires you completed assessed appraisals, depressive symptoms, and mindfulness. We also asked you questions regarding 'internalized stigma' (i.e., how much you believe stereotypes about depression, and if you believe that these stereotypes are true of yourself and experiences you've had). Finally, we asked you to provide information on the coping strategies you typically use, and how you tend to use these coping strategies across different types of situations. We are interested in learning if mindfulness is related to self-stigma among young adults. We are also interested in determining if mindfulness in itself can be used as a coping strategy, and if this type of coping strategy is more effective in certain types of situations. To assess this, we asked you to rate your use of various types of coping strategies in response to different kinds of stressful situations (i.e., workplace stress, financial stress, etc). We used these different sets of instructions in order to see if coping strategies were rated differently across a range of stressful situations.

\section{Why is this important to scientists or the general public?}

Previous research has shown that some individuals "self-stigmatize" (i.e., believe negative stereotypes about themselves) when they experience depressive symptoms. Furthermore, this type of stigma may have detrimental effects on health and self-appraisals, and might undermine individuals' efforts to cope with stressful situations. One variable, mindfulness (e.g., the ability to live "in the moment", and to observe situations in an objective, non-judgmental way), has been associated with more positive self-views, self-compassion and well-being. It is not known, however, if more mindful individuals experience stigma to a lesser extent, or if mindfulness itself can be used as a strategy for coping with different types of situations. If mindfulness and mindful coping are related to more positive appraisals, it might be helpful to focus on developing these skills in order to reduce stress and internalized stigma among young adults, and to improve their overall health and well-being.

\section{What are our hypotheses and predictions?}

We predict that individuals will have different levels of trait mindfulness. We also predict that mindfulness might be used as a coping strategy by some people, and that greater mindfulness (trait and state) will be associated with more positive self-appraisals, the use of active coping strategies (e.g., problem solving), lower levels of internalized stigma, and well-being.

\section{Is there anything I can do if I found this experiment to be emotionally upsetting?}

Yes. If you feel any distress or anxiety after participating in this study, please feel free to contact your family physician, the Mental Health Crisis Line (within Ottawa (613) 722-6914, outside Ottawa 1866-996-0991, Web Site: http://www.crisisline.ca/), or the Ottawa Distress Centre: (613) 238-1089, Web Site: www.dcottawa.on.ca 


\section{What if I have questions later?}

If you have any remaining concerns, questions, or comments about the experiment, please feel free to contact:

Kelly-Lyn Christie (Principal Investigator), at: klchrist@connect.carleton.ca (613-520-2600, ext. 6312)

Valerie Repta, at: vrepta@connect.carleton.ca (613-520-2600, ext. 7513)

Dr. Hymie Anisman (Faculty Sponsor), at: hanisman@connect.carleton.ca

Should you have any ethical concerns about this research, please contact Dr. Monique Sénéchal at: Monique_senechal@carleton.ca (613-520-2600 ext 1155). For other concerns, please contact Dr.

Janet Mantler (Chair, Department of Psychology, 613-520-2600, ext. 2648, psychchair@carleton.ca).

\section{Thank you for participating in this research!}




\section{Appendix I: Study 3.1 SONA Recruitment Notice}

\section{PART 1}

The purpose of this study is to investigate the relationship between personal characteristics and coping with various tasks. We are interested in examining how these personal characteristics influence the types of strategies people use when dealing with different types of challenging situations, as well as how these variables predict wellbeing. **THIS IS A TWO-PART STUDY** In PART 1, we are asking you to complete an online questionnaire to determine your eligibility for the laboratory session. This questionnaire will take approximately 5 minutes to complete. Once you have completed PART 1, you will be contacted by a researcher and notified about your eligibility to complete PART 2 of the study. PART 2 will take place approximately one week later. In this session, you will be asked to complete additional questionnaires and participate in a short task. We are interested in learning about how people respond to these tasks, and will be looking at differences in stress and immune hormones (i.e., cortisol). To measure these, we will be collecting blood samples during the second session. This procedure involves having a small needle placed in your arm to collect blood for the length of the task (approximately 50 minutes). All blood samples are collected by a Registered Nurse, and only a small amount of blood will be taken (approximately 1/18th of what would be collected by Canadian Blood Services if you went to donate blood). YOU WILL RECEIVE 2\% COURSE CREDIT OR \$20 FOR QUALIFYING AND PARTICIPATING IN THIS STUDY. You will register for PART 2 of the study AFTER you have completed PART 1 and your eligibility has been confirmed by the researcher.

Eligibility Requirements

TO BE ELIGIBLE FOR THIS STUDY, YOU MUST NOT HAVE A FEAR OF NEEDLES OR GIVING BLOOD, MUST NOT BE TAKING ANTI-INFLAMMATORY MEDICATIONS, AND MUST NOT HAVE A CHRONIC PHYSICAL CONDITION (E.G., DIABETES, HEART PROBLEMS, AUTOIMMUNE DISORDERS)

Duration

5 minutes

\section{PART 2}

The purpose of this study is to investigate the relationship between personal characteristics (e.g., coping strategies) and problem-solving. We are interested in examining how these personal characteristics influence the types of strategies people use when dealing with different types of challenging situations, as well as how these variables predict well-being. **THIS IS A TWO-PART STUDY. TO BE ELIGIBLE 
FOR THIS SESSION, YOU MUST HAVE COMPLETED PART 1**. In this session, you will be asked to complete questionnaires and participate in a short task. We are interested in learning about how people respond to different tasks, and will be looking at differences in stress and immune hormones (i.e., cortisol). To measure these, we will be collecting blood samples during the second session. This procedure involves having a small needle placed in your arm to collect blood for the length of the task (approximately 50 minutes). All blood samples are collected by a Registered Nurse, and only a small amount of blood will be taken (approximately 1/18th of what would be collected by Canadian Blood Services if you went to donate blood). YOU WILL RECEIVE 2\% COURSE CREDIT OR \$20 FOR PARTICIPATING IN THIS SESSION. Please only register for this session AFTER you have completed the first part of the study and the researcher has confirmed your eligibility.

Eligibility Requirements

TO BE ELIGIBLE, YOU MUST HAVE COMPLETED PART 1 OF THIS STUDY, MUST NOT HAVE A FEAR OF NEEDLES OR GIVING BLOOD, MUST NOT BE TAKING ANTI-INFLAMMATORY MEDICATIONS, AND MUST NOT HAVE A CHRONIC PHYSICAL CONDITION

Sign-Up Restrictions

Must have completed ALL of these studies:

-Personal Characteristics, Problem Solving and Coping with Challenging Tasks ("PART $1 ")$

Duration

90 minutes

Percentage

2 Percentage 


\section{Appendix J: Study 3.1 Informed Consent}

\section{General Consent}

The purpose of an informed consent is to ensure that you understand the purpose of the study and the nature of your involvement. The informed consent must provide sufficient information such that you have the opportunity to determine whether you wish to participate in the study.

This study has received clearance by the Carleton University Psychology Research Ethics Board (Ethics number: 11-056).

\section{Study Title: Personal Characteristics and Coping with Challenging Tasks (Laboratory} Session)

Research personnel. The following people are involved in this research project, and may be contacted at any time if you have questions or concerns:

Kelly-Lyn Christie, Principal Investigator (email: klchrist@connect.carleton.ca, phone: 613-520-2600, ext. 6312),

Jesse Howell, Graduate Researcher (email: jhowell1@connect.carleton.ca),

Dr. Hymie Anisman (Faculty Sponsor, email: hanisman@connect.carleton.ca),

Dr. Shawn Hayley (email: shawn_hayley@carleton.ca),

Dr. Kimberly Matheson (email: kim_matheson@carleton.ca).

Should you have any ethical concerns about this research, please contact Dr. Avi Parush, at : avi_parush@carleton.ca, (613-520-2600 ext. 6026). For any other concerns, please contact Dr. Anne Bowker (Chair, Department of Psychology, 613-520-2600 x. 8218, psychchair@carleton.ca).

Purpose. The purpose of this study is to investigate the relationship between personal characteristics (e.g., appraisals, coping strategies) and individual techniques for problemsolving and processing different types of tasks. We are interested in examining how these personal characteristics and appraisals influence the types of strategies people use when dealing with different types of stressful situations, as well as how these variables predict immune system functioning and well-being.

Task requirements. In this session we will be asking you to complete a short task, and to fill out a questionnaire package regarding different coping strategies you use, and depressive symptoms. We are also interested in examining differences in stress hormones and immune system functioning among participants. To measure these hormones, we will be asking you to consent to providing a set of five (5) blood samples during this session. This involves having a Registered Nurse insert a catheter into your arm (just as they do when you give blood at the doctor's or to Canadian Blood Services).

Over the course of the session, approximately $30 \mathrm{~mL}$ of blood will be taken (this amounts to about $1 / 18$ th of the amount taken by Canadian Blood Services). This portion of the study is entirely voluntary, and there is a separate consent form outlining the procedures for 
providing a blood sample. You will receive your choice of either $\$ 20$ OR 2\% course credit (to be counted towards your final grade if registered in PSYC 1001, PSYC 1002, PSYC 2001 or PSYC 2002) for participating in this portion of the study and providing a blood sample. At the end of this session you will be fully debriefed about this study and what the researchers are expecting to find. This session will take about 90 minutes to complete. This session will take place on campus at Carleton University.

Potential risk/discomfort. Some individuals might feel discomfort when asked to provide personal, sensitive information regarding medical history or depressive symptoms. If you feel any discomfort or distress, you may choose not to answer specific questions. You will not be penalized in any way if you do this. The debriefing form at the end of the study provides contact information for local support services that you may contact if you need or want help. Note: The following consent form describes the process for giving a blood sample.

Anonymity/Confidentiality. The data collected in this experiment are strictly confidential. All data are coded such that your name is not associated with the responses you provide. Any identifying information associated with your code will be confined to a single page that will be separated from your questionnaire, and kept in a separate, secured file by the research investigators, who will keep this information confidential.

Right to withdraw. Your participation in this study is entirely voluntary. At any point during the study, you have the right to not complete certain questions or to withdraw from the study without penalty.

\section{Signatures}

I have read the above form and understand the conditions of my participation. My participation in this study is voluntary, and I understand that if at any time I wish to leave the experiment, I may do so without having to give an explanation and with no penalty whatsoever. Furthermore, I am also aware that the data gathered in this study are confidential and anonymous with respect to my personal identity. My signature indicates that I agree to participate in this study.

Full Name (please print):

Participant Signature: Date: 


\section{Consent for Blood Collection and Analysis}

The purpose of an informed consent is to ensure that you understand the purpose of the study and the nature of your involvement. The informed consent has to provide sufficient information such that you have the opportunity to determine whether you wish to participate in the study.

This study has received clearance by the Carleton University Psychology Research Ethics Board (Ethics number: 11-056).

Study Title: Personal Characteristics and Coping with Challenging Tasks (Laboratory Session)

Research personnel. The following people are involved in this research project, and may be contacted at any time if you have questions or concerns:

Kelly-Lyn Christie, Principal Investigator(email: klchrist@connect.carleton.ca, phone: 613-520-2600, ext. 6312),

Jesse Howell, Graduate Researcher (email: jhowell1@connect.carleton.ca),

Dr. Hymie Anisman (Faculty Sponsor, email: hanisman@connect.carleton.ca),

Dr. Shawn Hayley (email: shawn_hayley@carleton.ca),

Dr. Kimberly Matheson (email: kim_matheson@carleton.ca).

Should you have any ethical concerns about this research, please contact Dr. Avi Parush, at : avi_parush@carleton.ca (613-520-2600 ext. 6026). For any other concerns, please contact Dr. Anne Bowker (Chair, Department of Psychology, 613-520-2600 x. 8218, ext. 1769,psychchair@carleton.ca).

Purpose and Task Requirements: Individuals experience a wide variety of events in their daily lives, however, perceptions of what is stressful may vary between people as well as between situations. When we experience stressful situations, our bodies react by producing various hormones and peptides to help us cope with the event. In order to assess these hormones, we would like to take a blood sample through a pump that slowly withdraws blood from your arm. This involves having a Registered Nurse insert a catheter into your arm (from the same area of your arm from which blood samples are normally drawn, just as they do when you give blood at a medical clinic or to Canadian Blood Services). Over the course of the session, approximately $30 \mathrm{~mL}$ of blood will be taken (this amounts to about 1/18th of the amount taken by Canadian Blood Services). All instruments used for blood withdrawal will be sterile and one-time use only. The blood samples will be analyzed for levels of stress related hormones (ACTH, cortisol, TNF- $\alpha$ ), oxytocin, serotonin, BDNF and cytokines (IL-6, IL-10). Once these markers have been analyzed, any remaining blood will be disposed of and will not be used for any other purposes. The total procedure will span 50 mins, and for agreeing to complete this portion of the study and provide a blood sample, you will receive your choice of $\$ 20$ or $2 \%$ course credit (to be counted towards your final grade if registered in PSYC 1001, PSYC 1002, PSYC 2001 or PSYC 2002). Your signature below indicates that you have given consent to have a blood sample taken for the purposes of this study. At the end of 
this session you will be debriefed about this study in its entirety, and what the researchers are expecting to find.

Potential Risk and Discomfort: Inserting the needle usually gives a small pin-prick (as it does when you give blood for testing or to Canadian Blood Services). In this case, however, the needle is taped down, covered, and left in place for 50 minutes while you will be engaged in the study. Most people don't notice the needle in their arm after a short adjustment period. However, if at any time you wish to stop the experiment, please let us know and the nurse will take out the needle.

Anonymity/Confidentiality: The sample you provide will be kept confidential. Your informed consent form will be separated from your questionnaire and kept in a separate and secured file by one of the research investigators who will keep this information confidential. The questionnaire and blood samples will be assigned the same code, and only this code will identify your samples. The blood samples will be stored in a secured storage area that is only accessible by the researcher and research assistants. Once these markers have been analyzed, any remaining blood will be disposed of and will not be used for any other purposes.

Right to Withdraw: Your participation in this study is entirely voluntary. At any point during the study you have the right to withdraw entirely without penalty.

\section{Signatures}

I have read the above description of the blood sample purpose and procedure. The data collected will be used in research publications and/or for teaching purposes. My signature indicates that I agree to provide a blood sample, and this in no way constitutes a waiver of my rights.

Full Name (please print):

Participant Signature:

Date:

Researcher Signature:

Date: 


\section{Appendix K: Study 3.1 Questionnaires}

\section{FFMQ}

Instructions: Please read each of the statements below, and indicate how true or untrue each statement is of you, using the scale provided. Please answer according to how you actually feel and act, and not how you would like to act. There are no right or wrong answers.

\section{Never/Very \\ Rarely true}

Very often/

Always true

1) I perceive my feelings and emotions without having to react to them.

2) I have trouble thinking of the right words to express how I feel about things.

3) In difficult situations, I can pause without immediately reacting.

$$
\begin{array}{llll}
1 & 2 & 3 & 4
\end{array}
$$

4) When I have a sensation in my body, it's hard for me to describe it because I can't find the right words.

$\begin{array}{lllll}1 & 2 & 3 & 4 & 5\end{array}$

5) I find myself doing things without paying attention

\begin{tabular}{|c|c|c|}
\hline 2 & 3 & 4 \\
\hline
\end{tabular}

7) I tell myself I shouldn't be thinking the way I'm thinking.

$1 \quad 2 \quad 3$

8) When I'm walking, I deliberately notice the sensations of my body moving.

$\begin{array}{llllll}1 & 2 & 3 & 4 & 5\end{array}$

9) I rush through activities without being really attentive to them.

$\begin{array}{lllll}1 & 2 & 3 & 4 & 5\end{array}$

10) I notice how foods and drinks affect my thoughts, bodily sensations, and emotions.

$\begin{array}{llllll}1 & 2 & 3 & 4 & 5\end{array}$

11) I can easily put my beliefs, opinions, and expectations into words.

$\begin{array}{lllll}1 & 2 & 3 & 4 & 5\end{array}$


12) I pay attention to sounds, such as clocks ticking, birds chirping, or cars passing.

$\begin{array}{lllll}1 & 2 & 3 & 4 & 5\end{array}$

13) I tell myself that I shouldn't be feeling the way I'm feeling.

$\begin{array}{lllll}1 & 2 & 3 & 4 & 5\end{array}$

14) I notice visual elements in art or nature, such as colors, shapes, textures, or patterns of light and shadow.

$\begin{array}{lllll}1 & 2 & 3 & 4 & 5\end{array}$

15) I think some of my emotions are bad or inappropriate and I shouldn't feel them.

16) I find it difficult to stay focused on what's happening in the present.

$\begin{array}{lllll}1 & 2 & 3 & 4 & 5\end{array}$

17) I can usually describe how I feel at the moment in considerable detail.

18) When I take a shower or a bath, I stay alert to the sensations of water on my body.

19) I do jobs or tasks automatically, without being aware of what I'm doing.

$\begin{array}{lllll}1 & 2 & 3 & 4 & 5\end{array}$

20) When I have distressing thoughts or images, I feel calm soon after.

$\begin{array}{lllll}1 & 2 & 3 & 4 & 5\end{array}$

21) When I do things, my mind wanders off and I'm easily distracted.

$\begin{array}{lllll}1 & 2 & 3 & 4 & 5\end{array}$

22) I don't pay attention to what I'm doing because

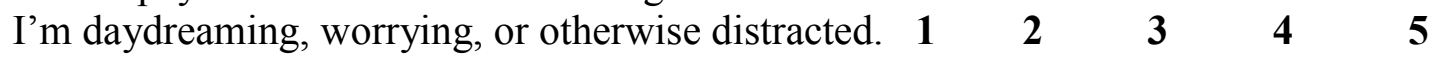

23) I make judgments about whether my thoughts are good or bad.

$\begin{array}{lllll}1 & 2 & 3 & 4 & 5\end{array}$

24) I'm good at finding the words to describe my feelings.

$\begin{array}{lllll}1 & 2 & 3 & 4 & 5\end{array}$

25) I pay attention to sensations, such as the wind in my hair or sun on my face.

$\begin{array}{lllll}1 & 2 & 3 & 4 & 5\end{array}$ 
Never/Very

Rarely true
Very often/ Always true

26) It's hard for me to find the words to describe what I'm thinking.

$\begin{array}{llllll}1 & 2 & 3 & 4 & 5\end{array}$

27) I watch my feelings without getting lost in them. $\begin{array}{lllllll} & \mathbf{1} & \mathbf{2} & \mathbf{3} & \mathbf{4} & \mathbf{5}\end{array}$

28) When I have distressing thoughts or images, I am able just to notice them without reacting.

29) Even when I'm feeling terribly upset, I can find a way to put it into words.

$\begin{array}{lllll}1 & 2 & 3 & 4 & 5\end{array}$

30) My natural tendency is to put my experiences into words.

$\begin{array}{lllll}1 & 2 & 3 & 4 & 5\end{array}$

31) It seems I am "running on automatic" without much awareness of what I'm doing.

$\begin{array}{lllll}1 & 2 & 3 & 4 & 5\end{array}$

32) I criticize myself for having irrational or inappropriate emotions.

33) I notice the smells and aromas of things.

34) I believe some of my thoughts are abnormal or bad and I shouldn't think that way.

35) I am easily distracted.

36) When I have distressing thoughts or images, I just notice them and let them go.

$\begin{array}{lllll}1 & 2 & 3 & 4 & 5\end{array}$

37) I pay attention to how my emotions affect my thoughts and behaviour.

38) I disapprove of myself when I have irrational ideas.

$\begin{array}{lllll}1 & 2 & 3 & 4 & 5\end{array}$

39) When I have distressing thoughts or images, I judge myself as good or bad, depending what the thought/image is about.

$\begin{array}{lllll}1 & 2 & 3 & 4 & 5\end{array}$ 


\section{PANAS}

Using the rating scale beside each item, please indicate how much each adjective describes how you feel at the moment. There are no right or wrong answers, we just want you to be as honest as possible in indicating how you're feeling right now.

\begin{tabular}{|c|c|c|c|c|c|c|c|c|}
\hline Active........Not at all & 0 & 1 & 2 & 3 & 4 & 5 & 6 & Extremely \\
\hline Afraid......... Not at all & 0 & 1 & 2 & 3 & 4 & 5 & 6 & Extremely \\
\hline Alert...........Not at all & 0 & 1 & 2 & 3 & 4 & 5 & 6 & Extremely \\
\hline Angry..........Not at all & 0 & 1 & 2 & 3 & 4 & 5 & 6 & Extremely \\
\hline Annoyed.... Not at all & 0 & 1 & 2 & 3 & 4 & 5 & 6 & Extremely \\
\hline Anxious...... Not at all & 0 & 1 & 2 & 3 & 4 & 5 & 6 & Extremely \\
\hline Ashamed......Not at all & 0 & 1 & 2 & 3 & 4 & 5 & 6 & Extremely \\
\hline Attentive...... Not at all & 0 & 1 & 2 & 3 & 4 & 5 & 6 & Extremely \\
\hline Confused......Not at all & 0 & 1 & 2 & 3 & 4 & 5 & 6 & Extremely \\
\hline Contempt.......Not at all & 0 & 1 & 2 & 3 & 4 & 5 & 6 & Extremely \\
\hline Depressed.....Not at all & 0 & 1 & 2 & 3 & 4 & 5 & 6 & Extremely \\
\hline Determined... Not at all & 0 & 1 & 2 & 3 & 4 & 5 & 6 & Extremely \\
\hline Disdain.......... Not at all & 0 & 1 & 2 & 3 & 4 & 5 & 6 & Extremely \\
\hline Disgust.........Not at all & 0 & 1 & 2 & 3 & 4 & 5 & 6 & Extremely \\
\hline Distressed.... Not at all & 0 & 1 & 2 & 3 & 4 & 5 & 6 & Extremely \\
\hline Embarrassed..Not at all & 0 & 1 & 2 & 3 & 4 & 5 & 6 & Extremely \\
\hline Enraged........Not at all & 0 & 1 & 2 & 3 & 4 & 5 & 6 & Extremely \\
\hline Enthusiastic...Not at all & 0 & 1 & 2 & 3 & 4 & 5 & 6 & Extremely \\
\hline Excited..........Not at all & 0 & 1 & 2 & 3 & 4 & 5 & 6 & Extremely \\
\hline Frustrated......Not at all & 0 & 1 & 2 & 3 & 4 & 5 & 6 & Extremely \\
\hline Guilty.......... Not at all & 0 & 1 & 2 & 3 & 4 & 5 & 6 & Extremely \\
\hline
\end{tabular}




\begin{tabular}{|c|c|c|c|c|c|c|c|}
\hline Happy......... Not at all 0 & 1 & 2 & 3 & 4 & 5 & 6 & Extremely \\
\hline Helpless....... Not at all 0 & 1 & 2 & 3 & 4 & 5 & 6 & Extremely \\
\hline Hostile.......... Not at all 0 & 1 & 2 & 3 & 4 & 5 & 6 & Extremely \\
\hline Humiliated.... Not at all 0 & 1 & 2 & 3 & 4 & 5 & 6 & Extremely \\
\hline Indifferent......Not at all 0 & 1 & 2 & 3 & 4 & 5 & 6 & Extremely \\
\hline Infuriated.... Not at all 0 & 1 & 2 & 3 & 4 & 5 & 6 & Extremely \\
\hline Inspired........ Not at all 0 & 1 & 2 & 3 & 4 & 5 & 6 & Extremely \\
\hline Interested..... Not at all 0 & 1 & 2 & 3 & 4 & 5 & 6 & Extremely \\
\hline Irritable....... Not at all 0 & 1 & 2 & 3 & 4 & 5 & 6 & Extremely \\
\hline Jittery .......... Not at all 0 & 1 & 2 & 3 & 4 & 5 & 6 & Extremely \\
\hline Nervous....... Not at all 0 & 1 & 2 & 3 & 4 & 5 & 6 & Extremely \\
\hline Proud.......... Not at all 0 & 1 & 2 & 3 & 4 & 5 & 6 & Extremely \\
\hline Regretful...... Not at all 0 & 1 & 2 & 3 & 4 & 5 & 6 & Extremely \\
\hline Responsible... Not at all 0 & 1 & 2 & 3 & 4 & 5 & 6 & Extremely \\
\hline Sad.............Not at all 0 & 1 & 2 & 3 & 4 & 5 & 6 & Extremely \\
\hline Scared.........Not at all & 1 & 2 & 3 & 4 & 5 & 6 & Extremely \\
\hline Strong......... Not at all 0 & 1 & 2 & 3 & 4 & 5 & 6 & Extremely \\
\hline Unhappy....... Not at all 0 & 1 & 2 & 3 & 4 & 5 & 6 & Extremely \\
\hline ......Not at all 0 & 1 & 2 & 3 & 4 & 5 & 6 & Extremely \\
\hline Worried.........Not at all 0 & 1 & 2 & 3 & 4 & 5 & 6 & Extremely \\
\hline
\end{tabular}




\section{STAI}

A number of statements which people have used to describe themselves are given below. Please read each statement and then circle the correct response to indicate how you feel right now, that is, at this moment. There are no right or wrong answers. Do not spend too much time on any one statement but give the answer which seems to describe your present feelings best.

Not at Somewhat Moderately Very
All
Much

1. I feel calm

1

2

3

4

2. I feel secure

1

2

3

4

3. I am tense

1

2

3

4

4. I feel strained

2

3

4

5. I feel at ease

2

3

4

6. I feel upset

1

2

3

4

7. I am presently

worrying over

possible misfortunes

2

3

4

8. I feel satisfied

2

3

4

9. I feel frightened

2

3

4

10. I feel comfortable 1

2

3

4

11. I feel self-confident 1

2

3

4

12. I feel nervous

2

3

4

13. I am jittery

2

3

4

14. I feel indecisive

2

3

4

15. I am relaxed

2

3

4

16. I feel content

2

3 
Not at Somewhat Moderately Very

Much

\begin{tabular}{|c|c|c|c|c|c|}
\hline 17. & I am worried & 1 & 2 & 3 & 4 \\
\hline 18. & I feel confused & 1 & 2 & 3 & 4 \\
\hline 19. & I feel steady & 1 & 2 & 3 & 4 \\
\hline 20. & I feel pleasant & 1 & 2 & 3 & 4 \\
\hline
\end{tabular}




\section{Experiment Evaluation}

We would like to make sure that you understood the nature of this study. To do so, we'd like you to complete the following questions. Point form responses are fine.

1. In your opinion, what was this study about?

2. What do you think we hope to find?

3. Is there anything about the conduct of this study that did not make sense to you (i.e., things the experimenters said or did, or questions we asked that seemed 'out of place'?) 


\section{Appendix L: Study 3.1 Debriefing}

\section{What are we trying to learn in this research?}

This research examines the relationship between mindfulness, self-stigma, coping strategies, physiological responses to stress, and depressive symptoms. The questionnaires you completed assessed depressive symptoms, coping strategies, mood, state anxiety and trait mindfulness. We also asked you to provide blood samples in order to assess how these variables influence cortisol (a stress hormone) and cytokines (proteins related to immune system functioning). We are interested in learning if mindfulness is associated with individual responses to stress and/or the use of specific coping strategies. To assess this, we asked you to complete a challenging task (giving a speech and completing a mental arithmetic exercise in front of a panel of graduate students). This task is meant to be challenging, and has been used in previous research to elicit stress hormones (e.g., cortisol). We asked you to complete this task in order to see if different levels of mindfulness influence how people respond to different types of stressful situations, and to see if cortisol and immune activity was lower among more "mindful" people.

\section{Why is this important to scientists or the general public?}

Previous research has shown a relationship between stress, depressive symptoms and immune system functioning. Less is known, however, about the specific factors that help to reduce these effects and promote well-being. Understanding individual difference variables (e.g., mindfulness) that might be associated with more adaptive responses to stress is important, since these responses may contribute to overall health. If mindfulness is shown to predict the use of effective coping strategies, positive appraisals and reduced stress, it might be helpful to teach these skills in order to improve physical health (i.e., immune system functioning) and well-being.

\section{What are our hypotheses and predictions?}

We predict that mindfulness (e.g., the ability to live "in the moment", and to observe situations in an objective, non self-judgmental way) will influence individuals' appraisals of different situations, and will also predict how well they are able to cope with stressful situations. We also predict that mindfulness will help to reduce physiological responses to stress (i.e., cortisol activity).

\section{Is there anything I can do if I found this experiment to be emotionally upsetting?}

Yes. If you feel any distress or anxiety after participating in this study, please feel free to contact the Carleton University Health and Counseling Services at: 613-520-6674, or the Distress Centre of Ottawa and Region at 613-238-3311 (http://www.dcottawa.on.ca).

\section{What if I have questions later?}

If you have any remaining concerns, questions, or comments about the experiment, please feel free to contact Kelly-Lyn Christie (Principal Investigator), at: klchrist@connect.carleton.ca (613-520-2600, ext. 7513), Jesse Howell, at: jhowell1@connect.carleton.ca, Dr. Hymie Anisman (Faculty Sponsor), at: hanisman@connect.carleton.ca, Dr. Shawn Hayley, at: shawn_hayley@carleton.ca, or Dr.

Kimberly Matheson, at: kim_matheson@carleton.ca. Should you have any ethical concerns about this research, please contact Dr. Avi Parush: Avi_Parush@carleton.ca (613-520-2600 ext 1155). For other 
concerns, please contact Dr. Anne Bowker (Chair, Department of Psychology, 613-520-2600, ext. 8218, psychchair@carleton.ca).

Thank you for participating in this research! 


\section{Appendix M: Study 3.2 Recruitment Email}

Dear (participant name),

You recently completed a study at Carleton University entitled Self-Appraisals and Situational Coping. At that time, you indicated that you would be interested in learning about future paid research studies taking place in our lab. The purpose of this email is to invite you to participate in a two-part study that we're conducting on personal characteristics and problem solving.

What would I have to do? In the first session, we are asking you to come in to the lab to participate in a short small-group training session (with approximately 5-7 other participants), which will take approximately 1.5 hours. Approximately one week later, we are asking you to return to the lab to complete a short questionnaire booklet and participate in a problem-solving task. We are interested in learning about how people respond to these events, including looking at differences in stress hormones (i.e., cortisol) and immune system functioning. To measure these variables, we will be collecting blood samples during the second session. This procedure involves having a small needle placed in your arm and taped down for the length of the task (approximately 45 minutes). All blood samples are collected by a Registered Nurse, and only a small amount of blood will be taken (approximately $1 / 18^{\text {th }}$ of what would be collected by Canadian Blood Services if you went to donate blood). If you agree to participate, you will be compensated $\$ 10$ for completing the first session, and you will be compensated an additional $\$ 20$ or $1.5 \%$ course credit (1.5\% increase in your final grade if registered in PSYC 1001, PSYC 1002, PSYC 2001 or PSYC 2002) for completing the second session and providing a blood sample.

If you would like to find out more or wish to participate in this study, please reply to this email to book an available timeslot.

Thank you,

(Researcher Name)

\section{This study has received clearance by the Carleton University Psychology Research Ethics Board (Ethics number : 11-056).}




\section{Appendix N: Study 3.2 Informed Consent (Session 1)}

The purpose of an informed consent is to ensure that you understand the purpose of the study and the nature of your involvement. The informed consent must provide sufficient information such that you have the opportunity to determine whether you wish to participate in the study.

This study has received clearance by the Carleton University Psychology Research Ethics Board (Ethics number: 11-056).

\section{Present study: Personal Characteristics and Problem Solving (Session 1)}

Research personnel. The following people are involved in this research project, and may be contacted at any time if you have questions or concerns:

Kelly-Lyn Christie (email: klchrist@connect.carleton.ca, phone: 613-520-2600, ext. 6312),

Warren Caldwell, Graduate Researcher (email: wcaldwel@connect.carleton.ca),

Jesse Howell, Graduate Researcher (email: jhowell1@connect.carleton.ca),

Keltie McDonald, Researcher (email: kmcdona3@connect.carleton.ca),

Dr. Hymie Anisman (Faculty Sponsor, email: hanisman@connect.carleton.ca),

Dr. Shawn Hayley (email: shawn_hayley@carleton.ca), or

Dr. Kimberly Matheson (email: kim_matheson@carleton.ca).

Should you have any ethical concerns about this research, please contact Dr. Monique Sénéchal, at : monique_senechal@carleton.ca (613-520-2600 ext. 1155). For any other concerns, please contact Dr. Anne Bowker (Chair, Department of Psychology, 613-520-2600, ext. 8218, psychchair@carleton.ca).

Purpose. The purpose of this study is to investigate the relationship between personal characteristics (e.g., appraisals, coping strategies) and individual techniques for problem-solving and processing different types of tasks. We are interested in examining how these personal characteristics and appraisals influence the types of strategies people use when dealing with different types of stressful situations, as well as how these variables predict well-being.

Task requirements. We are asking you to complete a small-group training session (with approximately 5-7 other participants) and to fill out short questionnaires regarding your background (e.g., demographics). At the end of this session, we will ask you to schedule a time with the researchers to complete the second portion of the study (approximately 1 week from now). The training session will take about 90 minutes to complete. You will receive $\$ 10$ for participating in this portion of the study. This session will take place on campus at Carleton University.

Potential risk/discomfort. There are no physical risks to participating in this study. Some individuals might feel uncomfortable when asked to provide personal information regarding medical history or current medications. If you feel any discomfort or distress, you may choose not to answer specific questions, and you will not be penalized in any way if you do this. The 
debriefing form at the end of the study provides contact information for local support services that you may contact if you need or want help.

Anonymity/Confidentiality. The data collected in this experiment are strictly confidential. All data are coded such that your name is not associated with the responses you provide. Any identifying information associated with your code will be confined to a single page that will be separated from your questionnaire, and kept in a separate, secured file by the research investigators, who will keep this information confidential.

Right to withdraw. Your participation in this study is entirely voluntary. At any point during the study, you have the right to not complete certain questions or to withdraw from the study without penalty.

\section{Signatures}

I have read the above form and understand the conditions of my participation. My participation in this study is voluntary, and I understand that if at any time I wish to leave the experiment, I may do so without having to give an explanation and with no penalty whatsoever. Furthermore, I am also aware that the data gathered in this study are confidential and anonymous with respect to my personal identity. My signature indicates that I agree to participate in this study.

Full Name (please print):

Participant Signature: Date:

Researcher Signature: Date: 


\section{Appendix O: Mindfulness Training Scripts \\ (Segal, Williams \& Teasdale, 2002) \\ Raisin Exercise}

I'm going to go around the room and give you each a few objects. Now, what I would like you to do is focus on one of the objects and just imagine that you have never seen anything like it before. Imagine you have just dropped in from Mars this moment and you have never seen anything like it before in your life.

NOTE: There is at least a 10 -second pause between phrases, and the instructions are delivered in a matter-of-fact way, at a slow but deliberate pace, asking the class to do the following:

Taking one of these objects and holding it in the palm of your hand, or between your finger and thumb. (Pause)

Paying attention to seeing it. (Pause)

Looking at it carefully, as if you had never seen such a thing before. (Pause)

Turning it over between your fingers. (Pause)

Exploring its texture between your fingers. (Pause)

Examining the highlights where the light shines...the darker hollows and folds. (Pause)

Letting your eyes explore every part of it, as if you had never seen such a thing before. (Pause)

And if, while you are doing this, any thoughts come to mind about "what a strange thing we're doing" or "what is the point of this" or "I don't like these", then just noting them as thoughts and bringing your awareness back to the object. (Pause)

And now smelling the object, taking it and holding it beneath your nose, and with each inbreath, carefully noticing the smell of it. (Pause)

And now taking another look at it. (Pause) 
And now slowly taking the object to your mouth, maybe noticing how your hand and arm know exactly where to put it, perhaps noticing your mouth watering as it comes up. (Pause)

And then gently placing the object in the mouth, noticing how it is "received," without biting it, just exploring the sensations of having it in your mouth. (Pause)

And when you are ready, very consciously taking a bite into it and noticing the taste it releases. (Pause)

Slowly chewing it, ...noticing the saliva in the mouth,...the change in consistency of the object. (Pause)

Then, when you feel ready to swallow, seeing if you can first detect the intention to swallow as it comes up, so that even this is experienced consciously before you actually swallow it. (Pause)

Finally, seeing if you can follow the sensations of swallowing it, sensing it moving down to your stomach, and also realizing that your body is now exactly one raisin heavier.

\section{Discussion questions:}

1. Does anyone want to say anything about their experiences while eating?

2. Did anyone notice anything different from the way you would normally eat? 


\section{Mindful Meditation Exercise}

1. Settle into a comfortable sitting position, either on a straight-backed chair or on a soft surface on the floor. If you use a chair, it is very helpful to sit away from the back of the chair, so that your spine is self supporting. If you sit on the floor, it is helpful if your knees actually touch the floor; experiment with the height of any cushions until you feel comfortably and firmly supported.

2. Allow your back to adopt an erect, dignified, and comfortable posture. If sitting on a chair, place your feet flat on the floor, with your legs uncrossed. Gently close your eyes.

3. Bring your awareness to the level of physical sensations by focusing your attention on the sensations of touch and pressure in your body where it makes contact with the floor or whatever you're sitting on. Spend a minute or two exploring these sensations.

4. Now bring your awareness to the changing patterns of physical sensations in the lower abdomen as the breath moves in and out of your body. (When you first try this practice, it may be helpful to place your hand on your lower abdomen and become aware of the changing pattern of sensations where your hand makes contact with your abdomen. Having "tuned in" to the physical sensations in this area in this way, you can remove your hand and continue to focus on the sensations in the abdominal wall.)

5. Focus your awareness on the sensations of slight stretching as the abdominal wall rises with each inbreath, and of gentle deflation as it falls with each outbreath. As best you can, follow with your awareness the changing physical sensations in the lower abdomen all the way through as the breath enters your body on the inbreath and all the way through as the breath leaves your body on the outbreath, perhaps noticing the slight pauses between one inbreath and the following outbreath, and between one outbreath and the following inbreath. 
6. There is no need to try to control the breathing in any way - simply let the breath come. As best you can, also bring this attitude of allowing to the rest of your experience. There is nothing to be fixed, no particular state to be achieved. As best you can, simply allow your experience to be your experience, without needing it to be anything other than it is.

7. Sooner or later (usually sooner), your mind will wander away from the focus on the breath in the lower abdomen to thoughts, planning, daydreams, drifting along - whatever. This is perfectly O.K. - it's simply what minds do. It is not a mistake or failure. When you notice that your awareness is no longer on the breath, gently congratulate yourself - you have come back and are once more aware of your experience. You may want to acknowledge briefly where the mind has been. Then, gently escort the awareness back to a focus on the changing patterns of physical sensations in the lower abdomen, renewing the intention to pay attention to the ongoing inbreath or outbreath, whichever you find.

8. However often you notice that the mind has wandered (and this will quite likely happen over and over again), as best you can, congratulate yourself on reconnecting with your experience in the moment, gently escorting the attention back to the breath, and simply resume following in awareness the changing pattern of physical sensations that come with each inbreath and outbreath.

9. As best you can, bring a quality of kindness to your awareness, perhaps seeing the repeated wanderings of the mind as opportunities to bring patience and gentle curiosity to your experience.

10. Continue with the practice for 15 minutes, or longer if you wish, perhaps reminding yourself from time to time that the intention is simply to be aware of your experience in each moment, as best you can, using the breath as an anchor to gently reconnect with the here and now each time you notice that your mind has wandered and is no longer down in the abdomen, following the breath. 


\section{3-Minute Breathing Space Exercise}

The first thing we do with this practice, because it's brief and we want to come into the moment quickly, is to take a very definite posture...relaxed, dignified, back erect but not stiff, letting our bodies express a sense of being present and awake.

\section{Minute 1.}

Now, closing your eyes, if that feels comfortable for you, the first step is being aware, really aware, of what is going on with you right now. Becoming aware of what is going through your mind; What thoughts are around? Here, again, as best you can, just noting the thoughts as mental events...so we note them, and then we note the feelings that are around at the present moment...in particular, turning toward any sense of discomfort or unpleasant feelings. So rather than try to push them away or shut them out, just acknowledge them, perhaps saying to yourself, "OK, that's how it is right now". And similarly with the sensations in the body...are there sensations of tension, of holding? And again, awareness of them, simply noting them. OK, that's how it is right now.

\section{Minute 2}

So, we've got a sense of what's going on right now. We've stepped out of automatic pilot. The second step is to collect our awareness by focusing on a single object - the movement of the breath. So now we really gather ourselves, focusing attention down in the movement of the abdominal wall...moment by moment, breath by breath, as best we can. So that you know when the breath is moving in, and you know when the breath is moving out. Just binding your awareness to the pattern of movement, gathering yourself, using the anchor of the breath to really be present.

\section{Minute 3}

And now as a third step, having gathered ourselves, we allow our awareness to expand. As well as being aware of the breath, we also include a sense of the body as a whole, so that we get a more spacious awareness. A sense of the body as a whole, including any tightness or sensations related to holding the shoulders, neck, back, or face...following the breath as if your whole body is breathing. Holding it all in this slightly softer, more spacious awareness. And when you are ready, just allowing your eyes to open. 


\section{Appendix P: Study 3.2 Analytic Problem Solving Script and Materials}

People have very different ways of approaching and solving problems, and analytical skills can be important in determining how quickly and effectively you solve problems. The purpose of this part of the study is to examine individual differences in analytical skills, and how this is related to problem solving. For this part of the study, we are going to ask you to complete a number of puzzles for approximately 30 minutes. We will also ask you to write a list of strategies you use to solve each type of puzzle. For the second part of the session, we will ask you to make a list of what you personally believe to be the most effective problem-solving strategies. You will have 30 minutes to complete the puzzles, and about 15 minutes to develop a list of the most effective problem solving strategies. Do you have any questions? 


\section{Participant \#}

\section{Problem-Solving Exercises}

Individuals encounter a number of problems and challenges every day, and different people might have very different ways of approaching and solving these problems. The purpose of this part of the study is 1) to help people to identify the specific problemsolving skills they already possess, and 2) to identify other skills that might be developed in order to enhance analytical and problem-solving ability.

On the following pages, you will find a series of 3 popular puzzle types. These puzzles range in length and difficulty. Please complete these puzzles to the best of your ability, paying attention to the specific strategies that you use to solve each type of problem. You will then be asked to answer a series of questions about the strategies that you used, and the strategies that were most effective for you. 
Instructions: Use the clues below to fill in the missing words in the puzzle to the right.

Please complete as much of the puzzle as you can, but do not spend more than 10 minutes on this puzzle

\section{Across}

1. From a lower to a higher position (8)

5. Guiding light (6)

9. Impoverished (8)

10. Flower part (6)

12. For all to see (5)

13. Healthy (9)

14. Foolishness (6)

16. Eternal (7)

19. Heavenward (7)

21. Studio couch (6)

23. Glaring (9)

25. An unborn baby (5)

26. Military greeting (6)

27. Feign (8)

28. Take back (6)

29. Eardrum (8)

\section{Down}

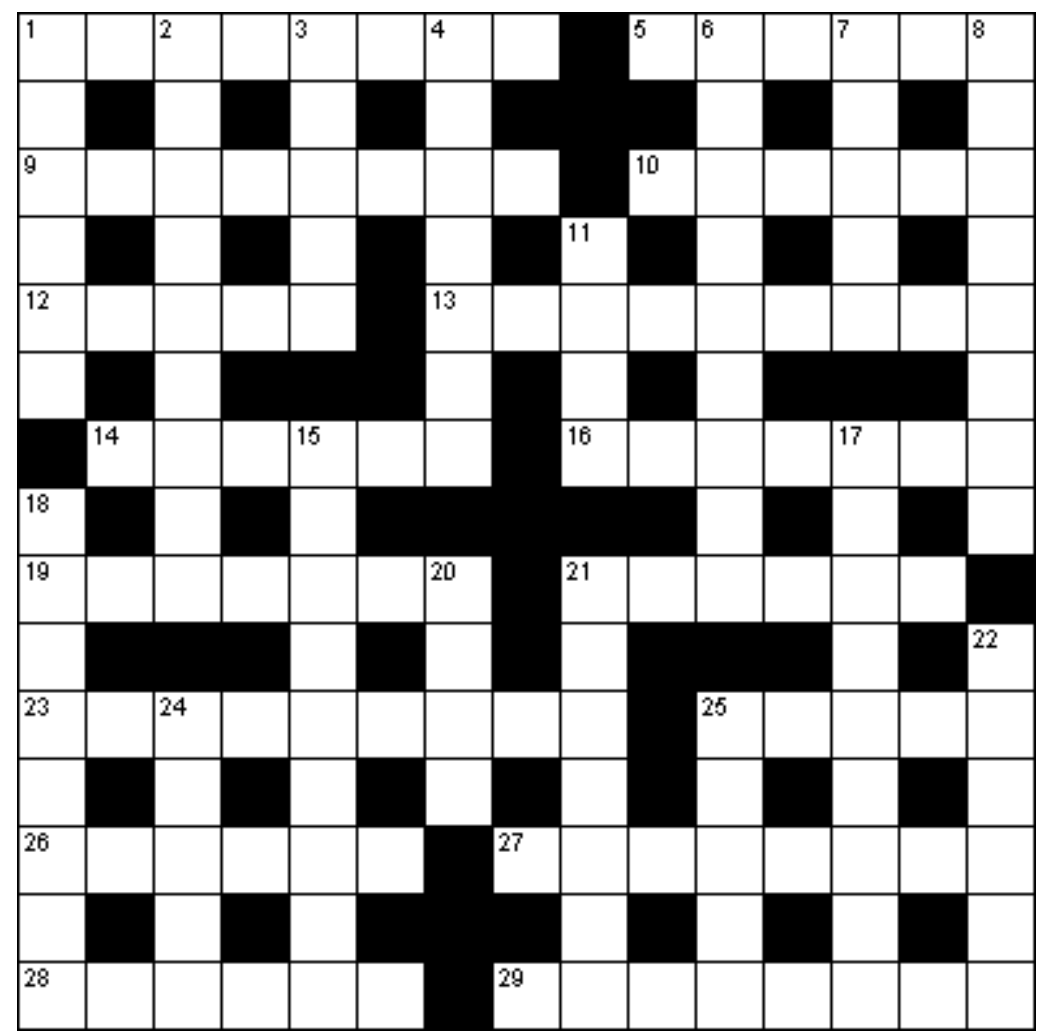

1. Agreement (6)

2. Day after Tuesday (9)

3. Not wrong (5)

4. Alleyway (7)

6. Very (9)

7. Jazz band (5)

8. Having no intelligible

18. Estimator for taxes (8)

20. Let go (4)

21. Fate (7)

22. High regard (6)

24. Museum piece (5)

25. A dull unattractive woman (5) meaning (8)

11. Coke or Pepsi (4)

15. Large long-armed ape

(9)

17. A tiredness of the eyes

(9) 
Instructions: Insert numbers in the boxes below, so that each row, column and $3 \times 3$ box contain the digits 1 through 9 exactly once.

Please complete as much of the puzzle as you can, but do not spend more than 10 minutes on this puzzle.

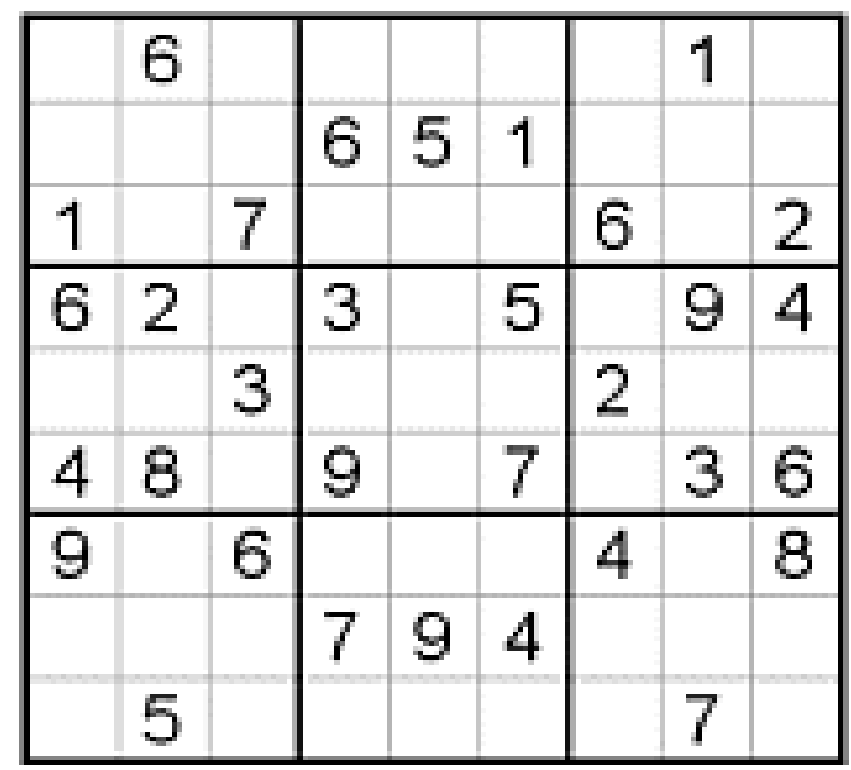

Instructions: Please read the following word problem, and record your answer in the space provided below. Please do not spend more than 10 minutes on this puzzle.

Two boxes are labelled "A" and "B". A sign on box A says "The sign on box B is true and the gold is in box A". A sign on box B says "The sign on box A is false and the gold is in box A". Assuming there is gold in one of the boxes, which box contains the gold?

Answer: 


\section{Assessment of Problem-Solving Skills}

Please take the next 15 minutes to think about the different strategies you used to solve each of the puzzles above (e.g., completing certain parts first, process of elimination, etc.), and answer the following questions.

1) In general, how would you rate the difficulty of these puzzles?

Very Easy

Somewhat Easy

Somewhat Difficult

Very

Difficult

2) Which specific puzzle did you find least difficult?
a. Crossword puzzle
b. Sudoku (number puzzle)
c. Word problem

3) Which puzzle did you find most difficult?
a. Crossword puzzle
b. Sudoku (number puzzle)
c. Word problem

4) Thinking about your problem-solving abilities right now, which of the following analytical skills and techniques do you feel you possess? (Select all that apply)

a. Organizational skills (time management, ability to delegate tasks to others, etc.)

b. Planning/decision making (deciding what steps need to be taken to solve a problem, prioritizing which steps need to be taken first, etc.)

c. Problem Identification (understanding the nature of a specific problem/task)

d. Logical thinking/reasoning

e. Brainstorming

f. Lexical decision making (word comprehension, vocabulary, choosing between sets of words) 
g. Lateral thinking (creative problem solving, ability to see problems in new ways)

h. Visualization

i. Reduction (breaking problems down in to smaller, solvable parts)

j. Gathering/analyzing information

k. Trial-and-error (testing possible solutions until the right one is found)

1. Mathematical skill (ability to perform calculations and solve mathematical problems)

m. Other (please specify):

5) Looking at the analytical skills and techniques that you did NOT select above, which of these would have helped you to solve these puzzles more effectively? (Select all that apply)

a. Organizational skills (time management, ability to delegate tasks to others, etc.)

b. Planning/decision making (deciding what steps need to be taken to solve a problem, prioritizing which steps need to be taken first, etc.)

c. Problem Identification (understanding the nature of a specific problem/task)

d. Logical thinking/reasoning

e. Brainstorming

f. Lexical decision making (word comprehension, vocabulary, choosing between sets of words)

g. Lateral thinking (creative problem solving, ability to see problems in new ways)

h. Visualization

i. Reduction (breaking problems down in to smaller, solvable parts)

j. Gathering/analyzing information

k. Trial-and-error (testing possible solutions until the right one is found)

1. Mathematical skill (ability to perform calculations and solve mathematical problems) 
m. Other (please specify):

6) Why do you think these strategies would have helped you to solve the puzzles more effectively?

7) Overall, which of these skills and techniques do you feel are most important for problem solving?

8) What specific steps could you take to develop or improve your analytic and problem solving skills? (please think of realistic, achievable ways that you might improve these abilities - e.g., taking specific classes, practicing, etc.)

9) Besides solving puzzles, do you think these skills would help you in other areas of your life (e.g., with school work, researching new things/ideas, defending arguments, etc.)? How? 


\section{Appendix Q: Study 3.2 Control Condition Script and Materials}

Many people participate in hobbies or extracurricular activities outside of school, such as participating in sports, playing music or volunteering. These activities can help people to feel happier or more balanced in their lives. We're interested in learning more about the kinds of activities that students are doing outside of school, and the effect that these activities have on well-being. In this part of the study, we will be asking you to choose an activity that you enjoy, and that you feel helps to describe who you are as a person. We'll be asking you answer a few questions about this activity and to write a little bit about your experiences.

Do you have any questions? 


\section{Participant \#}

\section{Personal Activities, Hobbies and Well-Being}

Many people participate in hobbies or extracurricular activities outside of school (e.g., participating in sports, playing music, volunteering, artistic pursuits such as photography, pottery, dance, writing, etc.). The purpose of this part of the study is to identify what kinds of activities students are doing outside of school, and to understand 1) why these activities are meaningful to students, and 2) how these activities contribute to well-being.

Please take a few minutes to think of an activity or hobby that you enjoy. This can be something that you're currently involved in, or an activity that you have done in the past that has been meaningful to you. On the next few pages, we will ask you to describe this activity, and to answer a few questions about it.

If you do NOT have a specific hobby, please think of an activity that you would like to do, or that you have been curious about trying. Please think about this activity when completing the following questions. 


\section{$\underline{\text { Personal Activities Exercise }}$}

Instructions: Please take the next $10-15$ minutes to think about a hobby or activity that is meaningful to you, and describe this activity in the space provided. Please describe both what the activity is, as well as why this activity is meaningful to you. There are no right or wrong answers. 


\section{Activity/Hobby Questionnaire}

Instructions: Please take the next 15 minutes to think about the activity you described above and answer the following questions.

1) When did you first start this activity/hobby (how old were you)?

2) Are you still involved in this activity?
a. YES
b. NO - I used to do this in the past, but I don't any more

If YES, approximately how many hours per week do you spend participating in this hobby (e.g., classes, rehearsal/practice, etc.)?
c. Less than 1 hour
d. More than 1 hour, but less than 5 hours
e. More than 5 hours, but less than 10 hours
f. More than 10 hours

3) What first motivated you to become involved in this activity (e.g., what were your reasons for starting)? 
4) If you are no longer involved in this activity, why did you stop?

5) People might enjoy hobbies for a number of reasons, including stress reduction or relaxation. Why do you enjoy the activity/hobby you described above? (Select all that apply)
a. Distraction from other things
b. Relaxation
c. Exercise
d. Social element/experience (e.g., spending time with teammates, etc.)
e. Provides a creative outlet
f. Helps to develop skills (e.g., athletic skills, musical ability, etc.)
g. Other (please specify):

6) How important do you feel it is for young people to have interests and hobbies outside of school?

Not important at all 
7) When you are/were participating in this activity, to what extent do/did you feel each of the following?

Not at All A Little Quite a Bit Very Much
a. Confident
1
2
3
4
b. Happy
1
2
3
4
c. Relaxed
1
2
3
4
d. Talented
1
2
3
4
e. Motivated
1
2
3
4
f. Unique
1
2
3
4
g. Focused
1
2
3
4

8) Has participating in this hobby has had a positive influence on other areas of your life (e.g., confidence with other tasks, physical strength, improved focus/attention, etc.)? How? 
9) Has participating in this hobby has had a negative influence on other areas of your life (e.g., injuries, taking time away from other tasks, etc.)? How? 


\section{Appendix R: Mindfulness Handouts}

\section{$\underline{\text { Automatic Pilot }}$}

\section{(Focus of the Raisin Exercise)}

In a car, we can sometimes drive for miles on "automatic pilot," without really being aware of what we are doing. In the same way, we may not be really "present," momentby-moment, for much of our lives: We can often be "miles away" without knowing it.

On automatic pilot, we are more likely to have our "buttons pressed": Events around us and our thoughts, feelings and sensations in the mind (of which we may be only dimly aware) can trigger old habits of thinking that are often unhelpful and may lead to worsening mood.

By becoming more aware our thoughts, feelings, and body sensations, from moment to moment, we give ourselves the possibility of greater freedom and choice; we do not have to go into the same old "mental ruts" that may have caused problems in the past.

The aim of these exercises is to increase awareness so that we can respond to situations with choice rather than react automatically. We do that by practicing to become more aware of where our attention is, and deliberately changing the focus of attention, over and over again.

\section{A Definition of Mindfulness:}

"Mindfulness means paying attention in a particular way: On purpose, in the present moment, and nonjudgmentally."

- Jon Kabat-Zinn (1990) 


\section{The Breath}

\section{(Focus of Mindful Meditation and the 3-Minute Breathing Space Exercise)}

Breath is life. You could think of the breath as being like a thread or a chain that links and connects all the events of your life from birth, the beginning, to death, the end. The breath is always there every moment, moving by itself like a river.

Have you ever noticed how the breath changes with our moods - short and shallow when we're tense or angry, faster when we're excited, slow and full when we're happy, and almost disappearing when we're afraid? It's there with us all the time. It can be used as a tool, like an anchor, to bring stability to the body and mind when we deliberately choose to become aware of it. We can tune into it at any moment during everyday life. Mostly, we're not in touch with our breathing - it's just there, forgotten. So one of the first things we do in mindfulness training is to get in touch with it. We notice how the breath changes with our moods, our thoughts, our body movements. We don't have to control the breath. Just notice it and get to know it, like a friend. All that is necessary is to observe, watch, and feel the breath with a sense of interest, in a relaxed manner.

With practice, we become more aware of our breathing. We can use it to direct our awareness to different aspects of our lives. For example, to relax tense muscles, or focus on a situation that requires attention. Breath can also be used to help deal with pain, anger, relationships or the stress in daily life. 


\section{Appendix S: Homework Exercises}

\section{Positive and Negative Events Calendar}

Name:

Instructions: Be aware of a pleasant event each day at the time it is happening. Use the following questions to help focus your awareness on the details of the experience as it is happening. Write it down later.

\begin{tabular}{|l|l|l|l|l|l|}
\hline & $\begin{array}{l}\text { What was the } \\
\text { experience? }\end{array}$ & $\begin{array}{l}\text { Were you aware of the } \\
\text { pleasant feelings while the } \\
\text { event was happening? }\end{array}$ & $\begin{array}{l}\text { How did your body feel, in } \\
\text { detail, during this } \\
\text { experience? }\end{array}$ & $\begin{array}{l}\text { What moods, feelings, and } \\
\text { thoughts accompanied this } \\
\text { event? }\end{array}$ & $\begin{array}{l}\text { What thoughts are in } \\
\text { your mind now as you } \\
\text { write this down? }\end{array}$ \\
\hline Example & $\begin{array}{l}\text { Going home at the end } \\
\text { of the day - heard a bird } \\
\text { sing. }\end{array}$ & Yes. & $\begin{array}{l}\text { Lightness across the face, } \\
\text { aware of shoulders dropping } \\
\text { and smiling. }\end{array}$ & $\begin{array}{l}\text { Relief, pleasure, thought } \\
\text { "it's so nice to be outside" }\end{array}$ & $\begin{array}{l}\text { It was a small thing, but } \\
\text { I'm glad I noticed it. }\end{array}$ \\
\hline Day 1 & & & & & \\
\hline Day 2 & & & & & \\
\hline Day 3 & & & & & \\
\hline Day 4 & & & & & \\
\hline Day 5 & & & & \\
\hline Day 6 & & & & \\
\hline Day 7 & & & & \\
\hline
\end{tabular}




\section{Analytic Thoughts Calendar}

Name:

Instructions: Be aware of a problem you encounter each day and how you solve it. Use the following table to record what the problem was, if you were able to solve it, and what strategies you used to accomplish this.

\begin{tabular}{|l|l|l|l|l|}
\hline & What was the experience? & $\begin{array}{l}\text { Were you able to solve } \\
\text { this problem? }\end{array}$ & $\begin{array}{l}\text { How did you handle } \\
\text { this problem? }\end{array}$ & $\begin{array}{l}\text { What specific strategies did you use to } \\
\text { solve this problem? }\end{array}$ \\
\hline Example & $\begin{array}{l}\text { Got an exam back - had a question marked } \\
\text { wrong that I thought I should get credit for. }\end{array}$ & Yes. & $\begin{array}{l}\text { Went to talk to the } \\
\text { professor after class. }\end{array}$ & $\begin{array}{l}\text { Thought about what I'd say, picked a } \\
\text { quiet time to talk to him } \\
\text { Thought about my answer again to make } \\
\text { sure I was right/confident }\end{array}$ \\
\hline Day 1 & & & & \\
\hline Day 2 & & & & \\
\hline Day 3 & & & & \\
\hline Day 4 & & & & \\
\hline Day 5 & & & & \\
\hline Day 6 & & & & \\
\hline Day 7 & & & & \\
\hline
\end{tabular}




\section{Appendix T: Study 3.2 Debriefing (Session1)}

\section{What are we trying to learn in this research?}

This research examines the relationship between personal characteristics (e.g., appraisals of stressful situations, coping strategies and depressive symptoms) and strategies for coping with various types of events. The training session you participated in today will allow us to look at individual differences in problem-solving and approaches to different situations, which we will be assessing in Session 2. We are interested in understanding how individuals learn various techniques, and how well these strategies can be applied to later events and tasks.

\section{Why is this important to scientists or the general public?}

Previous research has shown a relationship between personal characteristics, depressive symptoms and ways of coping with stress. Less is known, however, about specific strategies that individuals might use for coping with potentially stressful situations. Understanding individual difference variables that might be associated with more adaptive responses to stress is important, since these responses may contribute to overall health and well-being.

\section{Is there anything I can do if I found this experiment to be emotionally upsetting?}

Yes. If you feel any distress or anxiety after participating in this study, please feel free to contact the Carleton University Health and Counseling Services at: 613-520-6674, or the Distress Centre of Ottawa and Region at 613-238-3311 (http://www.dcottawa.on.ca).

\section{What if I have questions later?}

If you have any remaining concerns, questions, or comments about the experiment, please feel free to contact Kelly-Lyn Christie (Principal Investigator), at:

klchrist@connect.carleton.ca (613-520-2600, ext. 6312), Warren Caldwell, at: wcaldwel@connect.carleton.ca, Jesse Howell, at: jhowell1@connect.carleton.ca, Keltie McDonald, at: kmcdona3@connect.carleton.ca, Dr. Hymie Anisman (Faculty Sponsor), at: hanisman@connect.carleton.ca, Dr. Shawn Hayley, at: shawn_hayley@carleton.ca, or Dr. Kimberly Matheson, at: kim_matheson@carleton.ca. Should you have any ethical concerns about this research, please contact Dr. Monique Sénéchal at: Monique_senechal@carleton.ca (613-520-2600 ext 1155). For other concerns, please contact Dr. Anne Bowker (Chair, Department of Psychology, 613-520-2600, ext. 8218, psychchair@carleton.ca).

\section{Thank you for participating in this research!}




\section{Appendix U: Study 3.2 Informed Consent Forms (Session 2)}

\section{General Consent}

The purpose of an informed consent is to ensure that you understand the purpose of the study and the nature of your involvement. The informed consent must provide sufficient information such that you have the opportunity to determine whether you wish to participate in the study.

This study has received clearance by the Carleton University Psychology Research Ethics Board (Ethics number: 11-056).

\section{Present study: Personal Characteristics and Problem Solving (Session 2)}

Research personnel. The following people are involved in this research project, and may be contacted at any time if you have questions or concerns:

Kelly-Lyn Christie (email: klchrist@connect.carleton.ca, phone: 613-520-2600, ext. 6312),

Warren Caldwell, Graduate Researcher (email: wcaldwel@connect.carleton.ca),

Jesse Howell, Graduate Researcher (email: jhowell1@connect.carleton.ca),

Keltie McDonald, Researcher (email:kmcdona3@connect.carleton.ca),

Dr. Hymie Anisman (Faculty Sponsor, email: hanisman@connect.carleton.ca),

Dr. Kimberly Matheson (email: kim_matheson@carleton.ca )

Should you have any ethical concerns about this research, please contact Dr. Monique Sénéchal, at : monique_senechal@carleton.ca (613-520-2600 ext. 1155). For any other concerns, please contact Dr. Anne Bowker (Chair, Department of Psychology, 613-520-2600, ext. 8218, psychchair@carleton.ca).

Purpose. The purpose of this study is to investigate the relationship between personal characteristics (e.g., appraisals, coping strategies) and individual techniques for problem-solving and processing different types of tasks. We are interested in examining how these personal characteristics and appraisals influence the types of strategies people use when dealing with different types of stressful situations, as well as how these variables predict immune system functioning and well-being.

Task requirements. In this session we will be asking you to complete a problem-solving task, and to fill out a short questionnaire package regarding your experiences in the first portion of this study (e.g., the training session you completed), different coping strategies you use, and depressive symptoms. We are also interested in examining differences in stress hormones and immune system functioning among participants. To measure these hormones, we will be asking you to consent to providing a set of four (4) blood samples during this session. This involves having a Registered Nurse insert a catheter into your arm (just as they do when you give blood to Canadian Blood Services). Over the course of the session, approximately $24 \mathrm{~mL}$ of blood will be taken (this amounts to about $1 / 18^{\text {th }}$ of the amount taken by Canadian Blood Services). This portion of the study is entirely voluntary, and there is a separate consent form outlining the 
procedures for providing a blood sample. You will receive your choice of either \$20 OR 1.5\% course credit (to be counted towards your final grade if registered in PSYC 1001, PSYC 1002, PSYC 2001 or PSYC 2002) for participating in this portion of the study and providing a blood sample. At the end of this session you will be fully debriefed about this study and what the researchers are expecting to find. This session will take about 90 minutes to complete. This session will take place on campus at Carleton University.

Potential risk/discomfort. Some individuals might feel discomfort when asked to provide personal, sensitive information regarding medical history or depressive symptoms. If you feel any discomfort or distress, you may choose not to answer specific questions. You will not be penalized in any way if you do this. The debriefing form at the end of the study provides contact information for local support services that you may contact if you need or want help. Note: The following consent form describes the process for giving a blood sample.

Anonymity/Confidentiality. The data collected in this experiment are strictly confidential. All data are coded such that your name is not associated with the responses you provide. Any identifying information associated with your code will be confined to a single page that will be separated from your questionnaire, and kept in a separate, secured file by the research investigators, who will keep this information confidential.

Right to withdraw. Your participation in this study is entirely voluntary. At any point during the study, you have the right to not complete certain questions or to withdraw from the study without penalty.

\section{Signatures}

I have read the above form and understand the conditions of my participation. My participation in this study is voluntary, and I understand that if at any time I wish to leave the experiment, I may do so without having to give an explanation and with no penalty whatsoever. Furthermore, I am also aware that the data gathered in this study are confidential and anonymous with respect to my personal identity. My signature indicates that I agree to participate in this study.

Full Name (please print):

Participant Signature: Date:

Researcher Signature: Date: 


\section{Consent for Blood Collection and Analysis}

The purpose of an informed consent is to ensure that you understand the purpose of the study and the nature of your involvement. The informed consent has to provide sufficient information such that you have the opportunity to determine whether you wish to participate in the study.

Study title: Personal Characteristics and Problem-Solving

Research personnel. The following people are involved in this research project, and may be contacted at any time if you have questions or concerns:

Kelly-Lyn Christie (email: klchrist@connect.carleton.ca, phone: 613-520-2600, ext. 6312),

Warren Caldwell, Graduate Researcher (email: wcaldwel@connect.carleton.ca),

Jesse Howell, Graduate Researcher (email: jhowell1@connect.carleton.ca),

Keltie McDonald, Researcher (email: kmcdona3@connect.carleton.ca),

Dr. Hymie Anisman (Faculty Sponsor, email: hanisman@connect.carleton.ca),

Dr. Shawn Hayley (email: shawn_hayley@carleton.ca),

Dr. Kimberly Matheson (email: kim_matheson@carleton.ca).

Should you have any ethical concerns about this research, please contact Dr. Monique Sénéchal, at : monique_senechal@carleton.ca (613-520-2600 ext. 1155).

Purpose and Task Requirements: Individuals experience a wide variety of events in their daily lives, however, perceptions of what is stressful may vary between people as well as between situations. When we experience stressful situations, our bodies react by producing various hormones and peptides to help us cope with the event. In order to assess these hormones, we would like to take a blood sample through a pump that slowly withdraws blood from your arm. This involves having a Registered Nurse insert a catheter into your arm (from the same area of your arm from which blood samples are normally drawn, just as they do when you give blood to the Red Cross). Over the course of the session, approximately $25 \mathrm{~mL}$ of blood will be taken (this amounts to about $1 / 18^{\text {th }}$ of the amount taken by Canadian Blood Services). All instruments used for blood withdrawal will be sterile and one-time use only. The blood samples will be analyzed for levels of stress related hormones (ACTH, cortisol, norepinephrine, epinephrine, prolactin and oxytocin), and cytokines. Once these markers have been analyzed, any remaining blood will be disposed of and will not be used for any other purposes. The total procedure will span 45 mins, and for agreeing to complete this portion of the study and provide a blood sample, you will receive \$20. Your signature below indicates that you have given consent to have a blood sample taken for the purposes of this study. At the end of this session you will be debriefed about this study in its entirety, and what the researchers are expecting to find.

Potential Risk and Discomfort: Inserting the needle usually gives a small pin-prick (as it does when you give blood for testing or to Canadian Blood Services). In this case, however, the 
needle is taped down, covered, and left in place for 45 minutes while you will be engaged in the study. Most people don't notice the needle in their arm after a short adjustment period.

However, if at any time you wish to stop the experiment, please let us know and the nurse will take out the needle.

Anonymity/Confidentiality: The sample you provide will be kept confidential. Your informed consent form will be separated from your questionnaire and kept in a separate and secured file by one of the research investigators who will keep this information confidential. The questionnaire and blood samples will be assigned the same code, and only this code will identify your samples. The blood samples will be stored in a secured storage area that is only accessible by the researcher and research assistants. Once these markers have been analyzed, any remaining blood will be disposed of and will not be used for any other purposes.

Right to Withdraw: Your participation in this study is entirely voluntary. At any point during the study you have the right to withdraw entirely without penalty.

I have read the above description of the blood sample purpose and procedure. The data collected will be used in research publications and/or for teaching purposes. My signature indicates that I agree to provide a blood sample, and this in no way constitutes a waiver of my rights.

Full Name (please print):

Participant Signature: Date:

Researcher Signature: Date: 


\section{Appendix V: Study 3 Questionnaires}

\section{Background Information - Session 2}

The following questions are important for our analysis of stress hormones:

What time did you wake up this morning?

Are you currently being treated for any physical condition?

No___ Yes____ If yes, please specify

Are you taking any form of hormonal based contraception (birth control)?

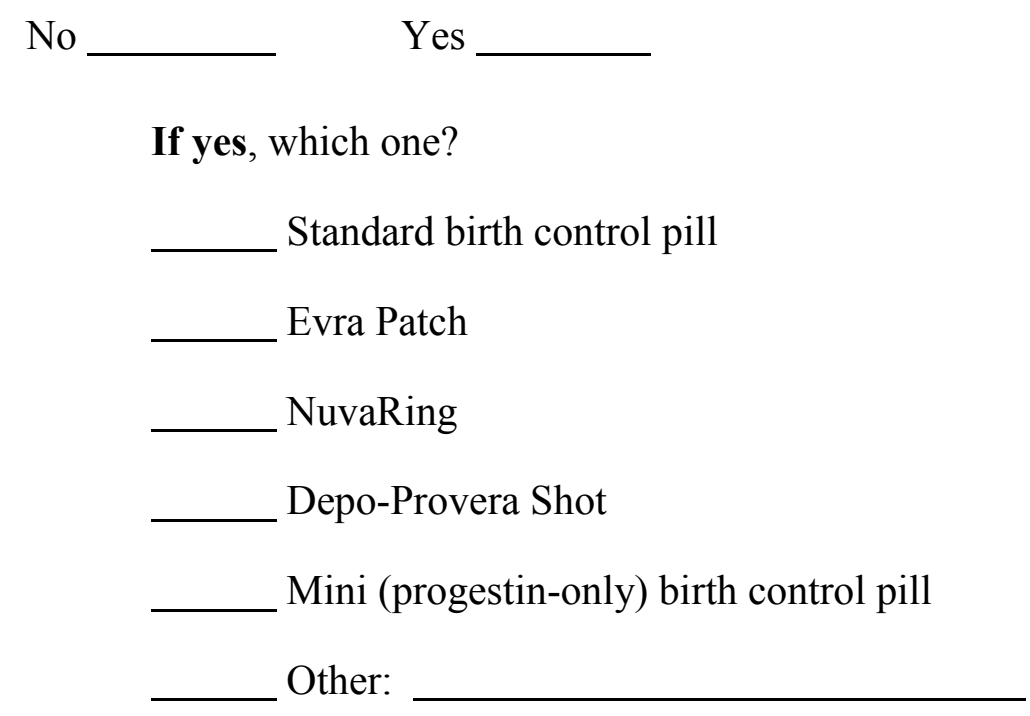

When did your last period begin?

And end?

Are you on any of the following medications (please check all that apply)?

Anti-inflammatories (please specify)

Anti-depressants (please specify)

Anti-anxieties (please specify)

Allergy medication (please specify)

Other prescription drugs (please specify) 
Have you taken any of these medications in the past 24 hours?

If yes:

Which medications?

What time did you take it/them?

How much did you take?

When was the last time you ate today?

What did you eat? 


\section{Session 1 Feedback Questionnaire}

The purpose of this questionnaire is to gain feedback on the training session you received last week. We are interested in learning your thoughts on this session so that we may identify areas of strength, as well as ways we might improve these exercises for future studies. Please answer the following questions as honestly as possible.

Thinking of the training session you participated in last week,

1. How interesting did you find this session to be?

Not at all

12
3
4

5
Extremely

6

7

2. How informative did you find this session to be?

Not at all

12

3

4

5

Extremely

6

7

3. How useful was this session for you?

Not at all

3

4

5

6

7

Additional Comments/Suggestions: 


\section{Employment Task (Stressor Condition)}

Please use the next two pages to write down any notes and organize your thoughts for your employability speech to the audience. Although you will not be able to use these notes during the speech, this may be a helpful organizational tool. Remember, you will be speaking to the audience as if you are applying for a job, and they are your potential employers. You will be judged on your performance. 


\section{Employment Task (Control Condition)}

Please use the next two pages to answer a few general questions about the types of work experience you may already have or may want, types of jobs you're interested in, which jobs you think are going to become more popular in the next 10 years, and general employment questions. You won't be marked or judged on any of your responses.

1. Do you currently have a job outside of school?

$$
\text { YES NO }
$$

b) If YES, how long have you been working at this job?

c) If YES, what are your main duties/responsibilities at this job?

2. What other job(s) have you had in the past? 
3. What jobs (either specific jobs or types of jobs) would you like to have in the future?

4. What jobs do you think are most popular/desirable for students today? Why? 
5. What jobs do you think are likely to become more popular for students 10 years from now? Why? 


\section{Appendix W: Study 3 Debriefing (Session 2)}

\section{What are we trying to learn in this research?}

This research examines the relationship between mindfulness, self-stigma, appraisals of stressful situations, coping strategies, physiological responses to stress, and depressive symptoms. The questionnaires you completed assessed depressive symptoms, appraisals, coping strategies and trait mindfulness. We also asked you to provide blood samples in order to assess how these variables influence cortisol (a stress hormone) and cytokines (proteins related to immune system functioning). We are interested in learning if mindfulness is associated with more positive appraisals and coping strategies. We are also interested in determining if mindfulness training helps individuals to cope with stress. To assess this, we randomly assigned half of the participants in this study to complete a mindfulness training session, and the other half to complete an analytic/problem-solving session. We then asked you to return to the lab for a second session, in which participants (again randomly selected) completed either a stressful task (giving a speech to a panel of graduate students) or a nonstressful task (a writing exercise). We used these different conditions in order to see if mindfulness training influenced how people responded to different types of stressful situations, and to see if cortisol and immune activity was lower among people who received mindfulness training.

\section{Why is this important to scientists or the general public?}

Previous research has shown a relationship between stress, depressive symptoms and immune system functioning. Less is known, however, about the specific factors that help to reduce these effects and promote well-being. Understanding individual difference variables (e.g., mindfulness) that might be associated with more adaptive responses to stress is important, since these responses may contribute to overall health. If mindfulness training is shown to predict the use of effective coping strategies, positive appraisals and reduced stress, it might be helpful to teach these skills in order to improve physical health (i.e., immune system functioning) and well-being.

\section{What are our hypotheses and predictions?}

We predict that mindfulness (e.g., the ability to live "in the moment", and to observe situations in an objective, non self-judgmental way) will influence individuals' appraisals of different situations, and will also predict how well they are able to cope with stressful situations. We also predict that mindfulness training will help to reduce physiological responses to stress (i.e., cortisol activity).

\section{Is there anything I can do if I found this experiment to be emotionally upsetting?}

Yes. If you feel any distress or anxiety after participating in this study, please feel free to contact the Carleton University Health and Counseling Services at: 613-520-6674, or the Distress Centre of Ottawa and Region at 613-238-3311 (http://www.dcottawa.on.ca).

\section{What if I have questions later?}

If you have any remaining concerns, questions, or comments about the experiment, please feel free to contact Kelly-Lyn Christie (Principal Investigator), at: klchrist@connect.carleton.ca (613-520-2600, ext. 6312), Warren Caldwell, at: wcaldwel@connect.carleton.ca, Jesse Howell, at: jhowe111@connect.carleton.ca, Keltie McDonald, at: kmcdona3@connect.carleton.ca),Dr. Hymie Anisman (Faculty Sponsor), at: hanisman@connect.carleton.ca, Dr. Shawn Hayley, at: 
shawn_hayley@carleton.ca, or Dr. Kimberly Matheson, at: kim_matheson@carleton.ca. Should you have any ethical concerns about this research, please contact Dr. Monique Sénéchal at: Monique_senechal@carleton.ca (613-520-2600 ext 1155). For other concerns, please contact Dr. Anne Bowker (Chair, Department of Psychology, 613-520-2600, ext. 8218, psychchair@carleton.ca).

\section{Thank you for participating in this research!}

